\title{
FERMILAB RESEARCH
}

PROGRAM

1976

EOITOR

J. LAGH

PRICE: $\$ 5.00$

Fermi National Accelerator Laboratory Batavia, Illinois

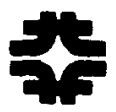


Introduction

This collection of one-page summaries of Fermilab proposals is intended to serve as a way station between the experiment number with its short title and the full proposal. It is not intended to be a review of the Fermilab experimental program. Just as an abstract of a journal article embodies the main points of the article, so these one-page summaries are intended to convey the major points of a proposal. These should include its physics justification, a brief description of the apparatus and the demands that the experiment will make on the Laboratory. Of course these summaries are not intended to take the place of the proposal itself which is the primary cocument available in the Fermilah library and at SLAC, BNL and CERN. Individual copies should be obtained from the spokesman of the experiment whose name is underlined in these sumnaries.

Summaries for all experiments and pending proposals are included. These comprise approved, unconsidered and deferred proposals. Pejected, withdrawn and inactive proposals are not included.

It is the experimenters themselves who are best able to write the surimary and in most cases that is what was done. For the early proposals and those cases where repeated cajoling could not procuce one from the experimenters, the sumary was prepared by a Fermilab staff member and then sent to the spokesman for comment. All proposals submitted before the liay 7,1976 deadine for consideration at the extended summer meetirs of cur Program Advisory Commiltee are included.

It is not intenced that this volume be updated annually but perhaps only reissued when the previous ones becones hopelessly cbsolete. 


\section{NAL NEUTRINO PROPOSAL}

A. Benvenuti, D. Cheng, D. Cline, W.T. Ford, R. Imlay, T.Y. Ling, A.K. Mann, F. Messing, J. Pilcher*, D.D. Reeder, C. Rubbia, R. Stefanski, L. Sulak

(Harvard-Pennsylvania-Wisconsin Collaboration)

This is a counter experiment in a broad band or monochromatic beam to study neutrino interactions with energies of 100 to $300 \mathrm{GeV}$ on free protons and heavy nuclei. The experimental apparatus consists of an $8 \mathrm{~m}$ long liquid hydrogen target; a target-calorimeter ( $3 \mathrm{~m} \times 3 \mathrm{~m} \times 8 \mathrm{~m}$ long) is a repetition of a basic module consisting of a lead target plate ( $6 \mathrm{~mm}$ thick), and a liquid scintillator (6mm thick). An interim pure liquid scintillation calorimeter has been installed and is working. The target-calorimeter has optical wide-gap spark chambers interspersed throughout. It is planned to install large drift chamber planes in the near future. If a wide band neutrino beam is used, then the neutrino energy is determined by the calorimeter and muon spectrometer. It is estimated that for the neutrino energy range $30 \mathrm{GeV}<\mathrm{E}_{\nu}<300 \mathrm{GeV}, \Delta \mathrm{E}_{V} / \mathrm{E}_{\psi} \leq \pm 0.1$. The muon spectrometer consists of four $18 \mathrm{~kg}$ iron core torodial magnets $3.6 \mathrm{~m}$ in diameter and $1.2 \mathrm{~m}$ long.

The physics objectives of the experiment are:

1) To search for the intermediate boson up to $W$ mass of $\simeq 30$ $\mathrm{GeV}$ at $400 \mathrm{GeV}$ operation of the accelerator. Both electronic and hadronic decays of the produced $w$ can be detected in the target-calorimeter or the resultant induced non locality will be detected.

2) To measure the cross section for the diagonal four-fermion interaction $\nu+z+\mu \mu \nu, z$. A $\sim 108$ cross section measurement can be made with 30-60 days of running time.

3) To measure the neutrino deep inelastic scattering cross section $d^{2} \sigma / d q^{2} d v$ and $\sigma_{\text {tot }}$ for $v_{\mu}+2 \rightarrow \mu+$ (anything) and $\bar{v}$ scattering. Tens of events per day with $q^{2}>200$ $(\mathrm{GeV} / \mathrm{c})^{2}$ are expected if scaling holds.

4) To measure_ $d^{2} \sigma / \mathrm{dq}^{2} \mathrm{~d} \nu$ and $\sigma_{t_{0}}\left(E_{\nu}\right)$ for the reaction $\nu_{\mu}+p \rightarrow \mu^{-}+$(anything). IE is in this phase of the experiment that the liquid hydrogen target is used. About 370 interactions per day are expected due to neutrinos above $45 \mathrm{GeV}$.

5) To look for hitherto unsuspected phenomena.

Summary reviewed by

D. Cline, February 1974

* Now at the University of Chicago 
STUDY OF MULTIPARTICLE $p-p$ AND $\pi-p$ INTERACTIONS

FROM $100 \mathrm{GeV} / \mathrm{C}$ TO $300 \mathrm{GeV} / \mathrm{C}$ WITH A 30-INCH BUBBLE

CHAMBER-WIDE GAP SPARK CHAMBER HYBRID SYSTEM

ANL, Duke Univ., Iowa State Univ., Univ. of Maryalnd, Michigan State Univ., Fermilab, Univ. of Notre Dame, Purdue Univ., Univ., of Toronto, Univ. of Wisconsin

\section{G. A. Smith (Spokesman)}

This group has been approved to study $p p$ and $\pi p$ interactions in an apparatus consisting of the $30^{\prime \prime} \mathrm{HBC}$ coupled with a wide-gap optical spark chamber detection system downstream of the bubble chamber. In addition to the approved 450,000 pictures, a total of 300,000 pictures have been recently approved (\#281) for later running. The detector system is designed to provide accurate bubble chamber data on all charged particles with momenta below - $20 \mathrm{GeV} / \mathrm{c}$, comparably accurate optical spark chamber data on downstream particles above $-20 \mathrm{GeV} / \mathrm{c}$ (typically 15\% FWHM on the momentum of a $200 \mathrm{GeV} / \mathrm{c}$ track), and information on neutral particles decaying into gamma rays. The aim of the experiment is to study single and multi-particle distributions from all final states (elastic and inelastic) produced at a variety of energies with a variety of bombarding particles in order to test models of current interest. The cost and construction of the entire system downstream of the bubble chamber has been assumed by the ANL-ISU-MarylandMSU-NAL members of the collaboration. The cost and construction of a rapid beam deflector and various beam diagnostic equipment, plus the measurement of the bubble chamber magnetic field, has been assumed by the remaining members of the collaboration.

First physics results on (a) two particle correlations and (b) inclusive and semi-inclusive diffraction have been published in two articles in Physics Letters [56B, 395 (1975) and 56B, 400 (1975)]. 
L. Alvarez, P. Eberhard, H. Oberlack, R. Ross, J.D. Taylor (University of California, Lawrence Berkeley Laboratory)

Objective: To find a magnetic monopole or decrease upper limits for monopole production cross section in a mass range where NAL experiments can do a mach better job than previous experiments. To conduct as foolproof a search as possible, e.g. one depending only on those monopole properties that are due to long range "Coulomb" like interactions and avoiding those involving short range interactions that are all questionable.

Technique: Expose a Cobalt coated aluminum target to $10^{18}$ (or more) protons at the highest energies available. Transport the target to Berkeley after exposure. Grind it and randomize the chips. Run samples separately in the superconducting electromagnetic monopole detector and measure their magnetic charge to a small fraction of a Dirac unit.

\section{Necessary conditions for finding a monopole in this search:}

Production: a) Mass $<15 \mathrm{GeV} / \mathrm{c}^{2}$ (500 GeV incident protons);

b) Production cross section $\geq 10^{-44} \mathrm{~cm}^{2}$ for $10^{18}$ interacting protons $\left(\sim 10^{-4}\right.$ of the presently known upper limits at $\mathrm{M}=15 \mathrm{GeV} / \mathrm{c})^{2}$.

Detection: a) Magnetic charge $>$ a small fraction of the Dirac charge, (1/100 of a Dirac charge is realizable). No upper limit. b) Induction of a signal in a coil (distances of several oentimeters). c) Binding of monopoles to the sample. If they are bound to aluminum nuclei the north and south poles must end up trapped at distances of more than 2 mils from one another. Either a small opening angle at production or multiple scattering according to theoretical predictions is sufficient. If they are not bound to aluminum nuclei the monopoles should be trapped in the ferromagnetic cobalt coat. Interaction distances involved in that case are over $1000 \AA$.

Justification: This is the only monopole search planned at NAL covering such a large range of magnetic charge and, after production, it depends only on the long range interactions of the monopole. Moreover, once we find a monopole, the sample is ready for immediate remeasurement since the monopole remains bound to the same piece of matter all the time.

Status: Irradiation of about $3 \times 10^{18}$ protons have been obtained. Search has been made. No monopole was found. Results have been published Phys. Rev. D11, 3099 (1975). Experiment is over. 
NEUTRON TOTAL CROSS SECTIONS UP TO $300 \mathrm{GeV}$

D. Burke, R. Gustafson, O. Haas, L. W. Jones, M. Longo,

J. Pluta, J. Stone, J. Chanowski (Univ. of Michigan);

B. Cork (LBI)

In this experiment measurements will be made of total cross sections for neutrons on hydrogen and heavier nuclei in the energy range 40 to $300 \mathrm{GeV}$ to an accuracy of $<28$. This will be done using a small-angle neutron beam and a standard transmission technique. The neutron detector is a total absorption spectrometer with an expected energy resolution $\approx \pm 58$. The apparatus is set up in the neutral beam enclosures upstream of the detector building of the Meson Area.

Summary reviewed by

M.J. Longo, February 1974 
A PROPOSAL TO MEASURE $\pi \pm_{p}$ AND $p-p$

DIFFERENTIAL ELASTIC SCATTFRING CROSS SECTIONS

FROM 50 to $170 \mathrm{GeV} / \mathrm{C}$

C. Akerlof, R. Kotthaus, R. Loveless, D. Meyer (University of Michigan); I. Ambats, T. Meyer, C. Ward (ANL); D. Eartly, D. Jovanovic, R. Lundy, S. Pruss (NAL); D. Rust (Indiana)

It is proposed to measure the differential cross sections for $\pi^{ \pm} \mathrm{p} \mathrm{K}^{ \pm} \mathrm{p}$ and $\mathrm{p} p$ elastic scattering in the range of $0.1<|-t|<$ 2 to $5(\mathrm{GeV} / \mathrm{c})^{2}$ from 50 to $280 \mathrm{GeV} / \mathrm{c}$. The apparatus consists of a liquid hydrogen target, a double arm spectrometer, wire spark chambers, an on-line computer, 3 Cerenkov counters in the incident beam, and 3 Cerenkov counters in the forward arm of the spectrometer. The highest attainable momentum and $t$ value will differ from particle to particle depending on the ratio of particles in the beam and the ability of the Cerenkov counters to separate efficiently. The angular and momentum measurements of the scattered particles will be used to provide a three constraint fit for every event: coplanarity, opening angle, and transverse momentum balance. The angular and momentum resolution in the forward arm spectrometer are $0.06 \mathrm{mrad}$ and $0.5 \%$ respectively at $200 \mathrm{GeV} / \mathrm{c}$, and $3 \mathrm{mrad}$ and 1.78 respectively at $2 \mathrm{GeV} / \mathrm{c}$ in the recoil arm spectrometer. The apparatus is designed to include sufficient rejection against inelastic processes to allow measurements down to cross sections at least as low as 100 nanobars/(GeV/c $)^{2}$.

The experiment will explore structure in the high momentum transfer region especially the $-t=1.4 \mathrm{GeV} / \mathrm{c}^{2} \mathrm{p}-\mathrm{p}$ dip bracketed by the CERN PS and ISR data and the dip in $\pi p$ at larger $t$. The slope parameter will be measured to an accuracy of 0.58 . Some inelastic data will automatically be collected. 
E. Behr, G. Bunce, R. Handler, R. March, P. Martin, G. Ott,

L. Pondrom, M. Sheaff,

w. Wilke (Univ. of Wisconsin);

A. Bersbach, T. Devlin, B. Edelman, R. Edwards, C. Muehleisen,

S. Yamin (Rutgers);

o. Overseth, P. Skubic (Univ.

of Michigan);

This experiment is proposed to make the following measurements in a $200 \mathrm{GeV}$ neutral hyperon beam:

1. Survey of a neutral hyperon beam, to measure production of $\Lambda^{\circ}, \bar{\Lambda} \circ, \Xi 0, \Xi 0, \mathrm{~K}_{1}^{\circ}, \mathrm{K}_{2}^{\circ}$, at 0,5 , and $10 \mathrm{mrad}$, by 300 $\mathrm{GeV} / \mathrm{c}$ protons on a copper target. The basic instrument consists of a spectrometer which looks at charged decay particles and is capable of a 18 mass resolution.

2. Measurement of $\Lambda \mathrm{N}$ total and diffraction elastic scattering cross sections in hydrogen and deuterium in the region 100 to $250 \mathrm{GeV}$. A slight modification of the spectrometer used in 1 . will be used for $\frac{d \sigma}{d t}$, and a transmission method will be used for the total cross section measurement. It may also be possible to make a measurement of the $\bar{\Lambda}{ }^{\circ} \mathrm{p}$ and $\bar{\Lambda}{ }_{\mathrm{d}}$ total cross section.

3. Search for the $\Delta S=2$ decay $\Xi^{\circ}+\mathrm{p}^{-}$, reducing the branching ratio limit from $\sim 10^{-3}$ to $\sim 10^{-7}$. At $10^{9}$ protons/pulse 72 hours are required. 
A STUDY OF NEUTRON-PROTON CHARGE-EXCHANGE SCATTERING IN THE MOMENTUM RANGE $50-300 \mathrm{GeV} / \mathrm{C}$

N. W. Reay, K. Reibel, T. A. Romanowski, N. R. Stanton (Ohio State Univ.); M. A. Abolins, M. T. Lin, G. A. Smith (Michigan State Univ.); K. W. Edwards (Carleton Univ.)

It is proposed to measure the neutron-proton chargeexchange cross section for incident neutron momenta between 50 and $300 \mathrm{GeV} / \mathrm{c}$, and four momentum transfers squared .002 $(\mathrm{GeV} / \mathrm{c})^{2}<|u|<1.0(\mathrm{GeV} / \mathrm{c})^{2}$.

The $\mathrm{np}$ charge-exchange cross section from .5 to $19 \mathrm{GeV} / \mathrm{c}$ is characterized by a sharp peak at $u=0$, with a width $\delta|\mathrm{u}| \simeq .02(\mathrm{GeV} / \mathrm{c})^{2}=\mathrm{M}_{\pi}{ }^{2}$, rising from a slowly falling background slope $4-6(\mathrm{GeV} / \mathrm{c})^{-2}$. Because of its width, the forward peak would seem to relate to pion exchange. However, the amplitude for pure pion exchange is constrained to vanish at $u=0$. Many models, both Regge and non-Regge, have been developed which are based on conspiracy or interference between the pion exchange amplitude and various poles or cuts. These models differ in their predictions about the dependence on incident momentum of the forward peak and the background slope.

The experiment is performed in an intense beam $\left(\sim 10^{7} / \mathrm{pulse}\right)$ of neutrons having a continuous energy spectrum which peaks near the end point of $300 \mathrm{GeV}$. Data are taken simultaneously over a wide band $(50-300 \mathrm{GeV})$ of the spectrum; this helps to minimize systematic errors in the energy dependence of the cross-section and to maximize the useful beam flux. Chargeexchange events are accumulated at the rate of approximately 0.15 per pulse. The direction and momentum of the fast forward outgoing proton are measured by a magnetic spectrometer and the velocity and direction of the recoil neutron are measured by a bank of neutron scintillators. Events are processed by an on-line computer. 
PROPOSAL TO STUDY INELASTIC HIGH ENERGY PROTON-PROTON

COLLISIONS IN THE DIFFRACTIVE REGION

P. Franzini, S. zubarik (Columbia), J. Lee-Franzini, J. Cole, P. Cowell (Stony Brook)

Summary:

Reactions of the type $\mathrm{p}+\mathrm{p}+\mathrm{x}+\mathrm{p}$ would be studied in the region where the recoil proton has momentum below $300 \mathrm{MeV} / \mathrm{c}$, where the mass of $x$ is between 1 and $10 \mathrm{GeV}$ and the $t$ range is from -0.1 to -0.001 . Several incident momenta would be used in $100 \mathrm{GeV}$ steps up to the highest attainable.

Carbon and polyethylene targets would be used in the first instance in the extracted beam. They would be mounted in a vacuum box which would also contain a series of 40 solid state counters to detect the recoil protons. The forward going particle would not be detected. A beam intensity of $10^{11}$ protons per pulse is needed. The detectors would measure the angle of the recoil proton to an accuracy of 10 milliradians; they would measure its energy to an accuracy of $50 \mathrm{keV}$. These yield mass resolutions of 80,25 and $20 \mathrm{MeV}$ at masses of 2,4 and $6 \mathrm{GeV}$ respectively. The momentum range of the recoil covered by the detectors would be 30 to about $300 \mathrm{MeV} / \mathrm{c}$ yielding a $\mathrm{t}$ range from -0.0009 to -0.073 $(\mathrm{GeV} / \mathrm{c})^{2}$.

Event rates per hodoscope bin of 10 to 1000 per $10^{13}$ incident protons are expected. The fraction of the beam lost in the target is about $10^{-7}$. $10^{4}$ events are desired at each energy and $10^{5}$ to $10^{6}$ at one energy. The former would require a few hours, the latter a few hundred. Total time requested is approximately 200 hours.

(JKW)

Received: June 1970 Approved: March 1971

Summary Revised: May 30, 1972 
F. Sciulli, B. Barish, D. Buchholz, T. Humphrey, F.s. Merritt, H. Suter (CaItech); G. Krafczyk, G. Fisk (NAL)

This experiment is a counter experiment in a monochromatic neutrino beam to study neutrino interactions on free protons and heavy nuclei. The neutrino beam momentum is variable from $80 \mathrm{GeV} / \mathrm{c}$ to $300 \mathrm{GeV} / \mathrm{c}$ with a momentum resolution of about \pm 68 . The experimental apparatus consists of a target-calorimeter and a muon spectrometer. The target-calorimeter ( $1 \mathrm{~m}^{2} \times 15 \mathrm{~m}$ long) is a sandwich of one interaction length plates of iron or lead, liquid scintillator detector and wire spark-chambers. A hydrogen target can also be used. The muon spectrometer consists of a $20 \mathrm{kG}$ iron core magnet ( $3 \mathrm{~m}$ diameter $\times 2 \mathrm{~m}$ long) between spark chambers.

Neutrino interactions in the targets produce "showers" (hadronic and electromagnetic) and muons. A rough determination of the shower energy by the calorimeter gives through the beam tune the neutrino energy. The muon direction and momentum are determined by the calorimeter wire chambers and muon spectrometer respectively.

The physics capabilities of the experiment are:

1. To detect the coherent production of intermediate vector bosons via $\nu+z \rightarrow W^{+}+H^{-}+Z^{\prime}$ if the $W$ mass is $15 \mathrm{GeV} / \mathrm{c}^{2}$ or less. The muonic decay mode of the $W$ is obseryed as a dimuon event with no hadronic cascade where the $\mu^{+}$has a large transverse momentum and the $\mu^{-}$has a low energy. The electron decay mode of the $W$ is observed as a single muon event accompanied by a relatively short range cascade. The hadronic decay mode of the $W$ is identified as a low energy peak in $\mathrm{d} \sigma / \mathrm{dE}_{\mu}$ for the event class $v+z+\mu^{-}+$hadrons.

2. To study the inelastic lepton scattering cross sections $\mathrm{d}^{2} \sigma / d q^{2} \mathrm{~d} \nu$ for $\nu+\mathrm{N} \rightarrow \mu^{-}+$(anything) in the region $0<q^{2}<600 \mathrm{GeV}^{2}$ and $0<\nu<300 \mathrm{GeV}$. A comparison with electron scattering data, and a test of the scaling hypothesis will be made. The inelastic cross sections for neutrino and anti-neutrino interactions will be measured over approximately the same energy range.

3. To measure the neutrino and antineutrino total cross sections on heavy nuclei from $80 \mathrm{GeV}$ to $300 \mathrm{GeV}$ neutrino energy. The departure from a linear rise of the total cross section with neutrino energy would signify a departure from the simple Fermi theory for scattering from a point particle. Such a saturation of the total cross section could be caused by the existence of $a \mathrm{~W}$ boson, higher order weak interaction effects, a nucleon form factor or other phenomena. 


\section{A SEARCH FOR MULTIGAMMA EVENTS} FROM MAGNETIC MONOPOLE PAIRS

G. B. Collins, J. R. Ficenec and W. P. Trower (Virginia Polytechnic Institute); J. Fischer and Seige Iwata (BNL)

The purpose of this experiment is to rediscover the multiphoton events found by Schein, Haskin and Glasser in cosmic rays. If they can be produced at $\mathrm{NAL}$, the next step would be to investigate the nature of their origin. Ruderman and Zwanziger suggested that the photons originate from the production and annihilation of bound magnetic monopole pairs.

In all, five such cosmic ray produced events have been reported. Al1 show multiplicities of 10 to 16 , total energies in the $10^{11} \mathrm{eV}$ range, angular divergences of $10^{-3}$ to $10^{-4}$ radians, and no charged particles accompanying the photons (photons are observed when they produce electron pairs in the photographic emulsion). These features have guided the design of our experiment. No charged particles means the photon jets must have been produced in a peripheral inferaction and at small angles to the incident particle. Also the $10^{11} \mathrm{eV}$ in the jet must approximate the incident energy. We note a similarity here with conventional electron pafr production and with muon pair production as observed at BNL by Christenson, et al. and attributed to virtual photons.

Our apparatus accordingly was designed to measure the multiplicity and angular distributions of simultaneous photons produced at small angles $\left(\sim 10^{-3} \mathrm{r}.\right)$ This has been accomplished by recording the position and number of unresolved electromagnetic showers produced by the photons in a $1 / 2$ radiation length converter placed in front of three $15^{\prime \prime} \times 15^{\prime \prime}$ proportional chambers. A veto counter array was placed in front of the converter to anti out any charged particles such as muons. A large number of neutrons accompany the photons and the interactions they produce in the converter have been avoided by recording only high multiplicity events with good time resolution $(0.1 \mu \mathrm{sec})$. We also included in the logic a threshold gas Cerenkov counter sensitive to high multiplicities of electrons and a lead glass counter with threshold set at $100 \mathrm{GeV}$ electromagnetic energy. The detecting apparatus was located in beam $\mathrm{M}-2$ over $100^{\prime}$ from a 0.1 collision length Be target $\left(10^{6}\right.$ incident protons of $\left.300 \mathrm{GeV}\right)$ to allow photons with $10^{-3} \mathrm{rad}$ angular spread to disperse.

We are presently analyzing some $10^{5}$ events satisfying our trigger conditions. Several interesting anomalies are observed in our data; however further analysis is required to determine whether they are instrumental or not.

Summary prepared by

G. B. Collins, Jan, 1975 


\section{TOTAL PHOTOABSORPTION CROSS SECIION AND A SEARCH FOR THE} PHOTOPRODUCED MONOPOLE AND OTHER UNUSUAL PARTICLES

D. Caldwell, J. Cumalat, A. Eisner, A. Lu, R. Morrison, F . Murphy, S. Yellin (Univ. of California at Santa Barbara); T. Nash (NAL): P. Davis, R. Egloff, G. Luste, J. Martin, J. Prentice (Univ. of Toronto)

The photon total cross section for hadronic final states produced from $\mathrm{H}$ and $\mathrm{D}$ is to be measured at photon energies from 14 to about $300 \mathrm{GeV}$ in $5 \%$ energy bins. Both the statistical accuracy and the systematic uncertainty are expected to be $< \pm 1 \%$. The $A$-dependence of the photoabsorption cross section is to be determined using $\mathrm{C}, \mathrm{Cu}$, and $\mathrm{Pb}$ targets at 60 and $170 \mathrm{GeV}$. In addition, there would be a simultaneous search for the Dirac monopole as a sudden increase in the total cross section at the monopole-pair production threshold, or by its manifestation as a multi-gamma shower. Also obtained simultaneously is information on forward events which have $\pi^{\circ} s$ or $\gamma^{\prime} s$, such as $\omega^{\circ} \rightarrow \pi 0^{\prime}+\gamma$.

The electron beam for this experiment has been tested and gives intensities within $30 \%$ of expectations. The photon beam provides tagged $\gamma$ energies in the range of $60 \%$ to $95 \%$ of the incident electron energy and has been used to observe $\psi$ photoproduction.

The photo-nuclear interaction will be identified by 1) the absence of high-energy photons or $e^{+}-e^{-}$particles emerging from the target at small angles, and 2) the appearance of hadrons or their decay products coming from the target at larger angles. In order to separate better $e^{f}-e^{-}$pairs from forward pion events and to detect multi-gamma showers, the shower veto counter is a hodoscope of lead-glass counters. Combined with proportional wire counter information, this makes possible the vetoing offline of electron pairs, using angle-energy correlations. The wide-angle hadron detectors also have position and energy determining capabilities.

The first measurements were made in the summer of 1975 with just the rear portion of the apparatus (lead-glass hodoscope and proportional wire counters) moved upstream to provide a larger solid-angle detector of $\boldsymbol{\gamma}^{\prime} s$ and charged particles. With the tagged photon beam we studied the photoproduction of the $\psi(3105)$ from $D$. 
HIGH MOMENTUM TRANSFER INELASTIC MUON SCATTERING AND TEST OF SCALE INVARIANCE AT NAL

C. Chang, K. W. Chen, D. Fox*, P. Kunz ${ }^{+}$(Michigan State Univ.); L. Hand, S. Herb, S. Loken, A. Russell Y. Watanabe (Cornell); M. Strovink (LBL); W. Vernon (U.C., San Diego)

The primary objective of the experiment is to test scale invariance using muon scattering from a high $\mathrm{z}$ target. Muon energy losses up to $180 \mathrm{GeV}$ and momentum transfers up to 100 $(\mathrm{GeV} / \mathrm{c})^{2}$ will be studied using a special technique of "scaling the apparatus". This physical scaling of the apparatus maps all experimentally obtained distributions into universal distributions, independent of the incident energy unless the scaling of muon interactions breaks down. It also drastically reduces the effect of systematic errors in the comparison of different energies.

The physics objective of this experiment is to look for evidence that a smaller structure exists within the neutron or proton.

If the nucleon consists of real, physical subparticles (partons) possessing strong interactions, these particles will be dressed by these interactions and possess a size 2 related to the interaction size, when expressed as a mass ${ }^{2}$ (in a parton form factor) to $100 \pm 50 \mathrm{Gev}^{2}$. In this experiment, if partons behave as mathematical point particles, the limit on $\mathrm{m}^{2}$ can be pushed back to $\sim 2000 \mathrm{GeV}^{2}$.

Data obtained in August and October, 1973 have been analyzed. This sample contains 100,000 triggers, at $150 \mathrm{GeV}$ from which were obtained about 40,000 scattering events. Also a small amount of data obtained at $56 \mathrm{GeV}$. The $150 \mathrm{GeV}$ data were compared with Monte-Carlo prediction using SIAC scaling structure function. Data when compared with prediction show a depletion in $Q^{2}$ distribution, amounting to about 408 at $\mathrm{Q}^{2}=40 \mathrm{GeV}^{2} / \mathrm{c}^{2}$. More data are being analyzed at this time.

The objectives remain to complete the experiment in two phases:

$$
\begin{aligned}
& \text { Phase (a) }: \begin{array}{l}
3 \times 10^{10} \text { muons incident }\left(500 \mathrm{hrs} \text { at } 10^{5}\right. \\
\mu / \text { pulse })
\end{array} \\
& \text { Phase (b) }: \begin{array}{l}
3 \times 10^{11} \text { muons incident }\left(500 \mathrm{hrs} \text { at } 10^{6}\right. \\
\mu / \text { pulse })
\end{array}
\end{aligned}
$$


PROPOSAL TO STUDY THE COHERENT DISSOCIATION OF NEUTRONS

J. Biel, T. Ferbel, P. Slattery, D. Underwood (Rochester);

E. Bleser (NAL); M. Block, B. Gobbi, L. Kenah, J. Rosen,

R. Ruchti (Northwestern); D. Freytag (Massachusetts)

At NAL energies, neutrons can be coherently excited by nuclei with appreciable cross section. By coherent it is meant that the target nucleus recoils as a single entity without nuclear excitation. This neutron excitation can occur either by $\gamma$ absorption in the nuclear coulomb field (Coulomb dissociation) or by Pomeranchukon exchange in the nucleus (diffractive dissociation). Both channels can result in the formation of $\pi^{\prime} p V^{\prime} s$. It is proposed to study such $V^{\prime} s$ using a spectrometer consisting of wire spark chambers, counters and an analyzing magnet.

The two coherent processes can be identified and distinguished by their characteristic variation with neutron energy, momentum transfer, angular correlation, effective mass and $A$ dependence. The former process (Coulomb) is dominated by $\Delta^{\circ}(1236)$ production while the latter is dominated by $N^{*}(1470)$ production. Coulomb dissociation is expected to be an order of magnitude more copious in high $z$ materials.

\section{The objectives of the experiment are:}

1. High statistical accuracy, comprehensive study of coulomb and diffractive dissociation. Variations in momentum transfer, angular correlations and invariant mass (up to $\sim 3 \mathrm{GeV}$ ) will be investigated as a function of target material and neutron energy $(50-300 \mathrm{GeV})$ using complex nuclear targets and hydrogen.

2. Observe coulomb and diffractive dissociation of the anti-neutron.

3. Using transmission targets, measure neutron total cross sections as a function of energy. 
SEARCH FOR HEAVY LEPTONS AND HADRONS AND HARD PENETRATING RADIATION IN THE NEUTRINO BEAM, STUDY OF DIFFRACTION SCATTERING OF NEUTRINOS; STUDY OF DEEP INELASTIC $\nu_{\mu}$ SCATTERING IN A Ne BUBBLE CHAMBER AT FERMILAB, AND TEST OF THE $\Delta S=\triangle Q$ RULE AT HIGH MOMENTUM TRANSFER USING INCLUSIVE REACTIONS

A. Benvenuti, U. Camerini, W. Fry, J. Mapp, R. March, J. Michael (Univ. of Wisconsin); H. Wachsmuth (CERN)

An experiment has been proposed for the Fermilab 15-foot bubble chamber with a neon (or neon-hydrogen) filling. The specific approval is for $50 \mathrm{~K}$ pictures for each of two proton targeting methods which are described below with the major physics interests.

I. Targeting of protons at the beginning of the muon shield (with no focusing)

Search for $\lambda$ by looking for $v_{\lambda}$ interactions. $\lambda=$ heavy lepton $(M=3 \mathrm{GeV}$ assumed). These could be produced directly $(\lambda \bar{\lambda})$ in hadron collisions or by protoproduction. They would be identified by :

$$
\begin{aligned}
& v_{\lambda}+n+\lambda^{-}+\text {anything } \\
& v_{\lambda}+p \rightarrow \lambda^{+}+\text {anything } \\
& \text { and } \lambda \pm \rightarrow \pi^{ \pm} v_{\lambda}
\end{aligned}
$$

$\lambda$ will be identified by studying the characteristics of the decay. A comparison will be made with $\nu_{\mu}$ interactions. This experiment is similar to Mann and Schwarz experiment at SLAC.

II. Conventional targeting of the proton beam with broad band focusing of the secondary hadrons.

a) Measure of charge radius of $v_{\mu}$ through muonless events; i.e.

$$
\begin{aligned}
v_{\mu}+p+v_{\mu}+p \\
\rightarrow \nu^{+}+\text {anything }
\end{aligned}
$$

e.g. $\quad \frac{d \sigma(v p \rightarrow v p \pi)}{d \sigma(e p+e p \pi)}=\frac{q^{4}}{30}\left\langle r^{2}\right\rangle^{2}$

where $\left\langle r^{2}\right\rangle$ is $v_{\mu}$ charge radius.

b) Diffraction dissociation of $\nu_{\mu}$ e.g. $v_{\mu}+z \rightarrow z+\mu^{+} \mu-v_{\mu}$

c) Deep inelastic scattering. Measure $E_{\nu}, v, q^{2}$. It is possible to measure $E_{v}$ by using a short fiducial volume and measuring all particles belonging to the interaction ( $\pi^{\circ}$ 's etc.). Neon has advantage of $\pi^{\circ}$ detection and enhancing diffraction dissociation.

III. Search for Charm 
PROPOSAL TO INVESTIGATE ANTINEUTRINO INTERACTIONS IN HYDROGEN AT NAI

Y. Cho, M. Derrick, T. Fields, I. Hyman, B. Musgrave,

P. Schreiner, H. Yuta (ANL); A. Engler, G. Keyes,

R. W. Kraemer (Carnegie-Mellon Univ.)

It is proposed to study high energy $\vec{v}_{\mu}$ interactions on hydrogen in the NAL 15-foot bubble chamber. The anti-neutrinos are produced from a horn-focused hadron beam produced with protons of energy $>200 \mathrm{GeV} / \mathrm{c}$. The availability of a mugn identifier system would be helpful. An exposure of 1019 interacting protons, or $1,000 \mathrm{~K}$ pictures ( $80 \mathrm{~K}$ events) are requested.

Physics motivation resides in the following:

1. Specific channels such as

a. Elastic scattering $\bar{\nu}_{\mu}+\mathrm{p}+\mu^{+}+\mathrm{n}$. Can probably only use events where $n$ scatters ( -5000 events). The neutron scatters will also measure the baryon polarization and so constrain the form factors.

b. Hyperon production - tests cabibbo and other theories.

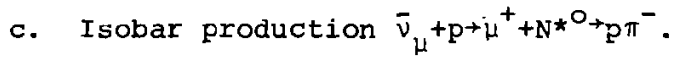

d. Other fittable final states such as $\bar{v}+p \rightarrow \mu^{+}+p+\pi^{-}+\pi^{+}+\pi^{-}$, etc.

2. Search for $\Delta S=2$ transitions and other reactions forbidden by the usual conservation laws

$$
\begin{aligned}
& \bar{v}+\mathrm{P}+\mathrm{H}^{+}+\mathrm{E}^{\mathrm{O}} \\
&+\mathrm{H}^{+}+\Sigma^{+}+\mathrm{K}^{-} .
\end{aligned}
$$

3. Characteristics of deep inelastic scattering
a. o Total vs. $\mathbf{E}_{\bar{v}}$.
b. $\mathbf{x}$ and $y$ distribution of inelastic events.
c. Contribution of specific channels to the different kinematic regions.


TEST AND CALIBRATE A LARGE NaI (Tl) TANC DETECTOR AND TO MEASURE NEUTRAL HADRON TOTAL CROSS SECTIONS

B. Beron, B. Chambers, J. F. Crawford, I. Doster, R. Ford, R. Hofstadter, E. B. Hughes, P. Lecoultre, L. O'Neill, R. Parks, R. F. Schilling, J. Vanderlans (Stanford Univ.)

They propose to test and calibrate a NaI(Tl) Total Absorption Nuclear Cascade (TANC) detector. This could later be used as a neutral particle detector with both high detection efficiency and good energy resolution. In order to determine the energy resolution of the detector they need a beam with a small ( . . to .28 $\Delta p / p)$ momentum bite. They desire to run protons at several energies up to the maximum attainable and to run pions if possible. An intensity of $10^{2}$ particles/second would probably be adequate. On the other hand, they should be able to make use of an instantaneous rate of $\sim 5 \times 10^{5} /$ second.

Their time request is for "several days, not necessarily in one continuous run". Their space requirements are for 10 feet along the beam line and space nearby for a moderate amount of electronics which they will bring with them. They further propose to measure total cross sections for neutrons and $K_{\mathrm{L}}^{O}$ using this detector. The details of this second phase will, of course, depend on the results of Phase $I$.

Summary reviewed by

R. Hofstadter, February 1974 
R. W. Huggett, D. L. Cheshire, W. V. Jones, P. N. Kirk (Iouisiana State Univ.); K. Pinkau, W.K.H. Schmidt, M. Simon (Max Planck Institute)

The results of this experiment will be used to interpret data from ionization spectrometers used in cosmic ray balloon experiments and in NAL experiments. The results will also be used in the design of an ionization spectrometer to be carried on one of the first space Shuttle missions. The objectives of this experiment are:

1. Investigate both the longitudinal and lateral development of nuclear electromagnetic cascades in tungsten and CsI.

2. Test the use of plastic scintillator wedges for determining the lateral position of cascades in tungsten.

3. Determine the fraction of the primary energy going into a) neutral pions, and b) disintegration of the target nucleus.

4. Measure the inelastic cross section.

The experiment was completed in June of 1974. Data was taken with protons at 100, 200, and $300 \mathrm{GeV}$ and with pions at $200 \mathrm{GeV}$. The total beam time used was 50 hours.

Summary revised by

R. W. Huggett, January 1976 


\section{A PROPOSAL TO STUDY SMALL ANGLE $\mathrm{p}-\mathrm{p}$ SCATTERING AT VERY HIGH ENERGIES}

I. H. Chiang, A. Melissinos (Rochester); R. Carrigan, E. Malamud,

R. Yamada (NAL); R. Cool, D. Goulianos, S. Olsen (Rockefeller); Plus USSR Participants

\section{Summary:}

The experiment uses a thin foil and a hydrogen jet target to measure small angle p-p elastic and inelastic scattering. By detecting the slow recoil proton studies of the energy dependence of the slope of the p-p diffraction peak will be made. Measurement in the coulomb interference region will give the real part of the nuclear scattering amplitude. Inelastic scattering will produce nucleon isobars.

The target would be placed in the internal circulating beam of the accelerator and measurements would be made during the acceleration cycle from $7 \mathrm{GeV}$ to the top energy available. Solid state detectors (10) and a sodium iodide crystal would be placed 2.5 meters from the target near $90^{\circ}$ to the beam Iine to detect the recoil protons. This system would measure the slope of the diffraction peak from $t=-.001$ to $-.25(\mathrm{GeV} / \mathrm{C})^{2}$ to an accuracy of \pm 18 . The ratio of the real to imaginary part of the scattering amplitude would be measured in sign and magnitude to an accuracy of 0.01 to 0.02. In inelastic scattering, the missing mass distribution of the recoils will detect isobars.

Event rates of 25 per pulse are expected from a beam of $10^{12}$ protons. Estimated running time is about 20 days. Increasing efforts are being made to measure reliably the s-dependence of the extrapolated to $t=0$ differential cross section. (JKW)

Received: June 15, 1970 Approved: February 1971 Summary Revised: May 30, 1972 
MULTIBODY FINAL STATES IN pP COLLISIONS UP TO $500 \mathrm{GeV} / \mathrm{c}$

E. Malamud, D. Gordon, J. Lach, (NAL) P. Schlein, W. Slater, (University of California at Los Angeles)

\section{Summary:}

This proposal requests 100,000 pictures at the hiahest available proton energy with three or more tracks/picture. The number of tracks/picture will be determined by scanning early film. If a higher flux can be used they may request some of their pictures be taken at another energy. In addition to scanning all the film quickly in a search for new particles, they will study the statistical properties of inelastic pp collisions by measuring the relatively slow particles produced in the backward hemisphere in the c.m. The data from this proposal could be used in the design of the multiparticle detector system of proposal 110, which some of the proposers of this experiment are working on.

(FRH)

Received: June 15, 1970

Sumnary Revised: May 30, 1972

\#37 is for $15^{\prime}$ bubble chamber

\#37A is for 30" bubble chamber

8/26/71 37A approved pp 50K highest. 
Proposal to Study Neutrino Interactions with Protons Using the 15-foot Bubble Chamber at NAL

J. P. Berge, D. Bogert, F. A. DiBianca, R. Hanft, C. Kochowski,

F. A. Nezrick, Y. Ryabov, W. G. Scott, W. Smart (Fermilab);

R. J. Cence, F. A. Harrís, S.I. Parker, M. W. Peters, V. Z.

Peterson, V. J. Stenger (U of Hawaii); A. Barbaro-Galtieri, G. R. Lynch, J. P. Marriner, F. T. Solmitz, M. I. Stevenson, (LBI); J. W. Chapman, C. T. Coffin, R. N. Diamond, H. T.French, W. C. Iouis, B. P. Roe, A. A. Seidl, D. Sinclair, J. C. Vander Velde (U. of Michigan).

This experiment uses the $15^{\prime}$ bubble chamber filled with hydrogen exposed to a broad-band horn focused neutrino beam produced by $300 \mathrm{GeV}$ protons. An EMI would be used if it existed. 300,000 pictures have been approved which should yield about 15,000 events for $5 \times 10^{12}$ protons per pulse on the horn target. A large physics program can be accomplished without serious muon ambiguities even without the EMI information. A detailed neutrino flux calibration procedure is proposed which depends on measuring the $\pi$ and $K$ yields from the neutrino target as well as the muon distribution in the shield. This group has constructed the muon monitoring system in the shield and is involved in measuring the neutrino target meson yields using the muon beam line in the neutrino area.

This proposal is a detailed study of the individual channels produced in neutrino-proton interactions. The physics objectives of this experiment are:

1. New particle search--charm, quarks, monopoles, etc.

2. W-boson search via hadron and electron decay.

3. Four-fermion interactions.

4. Single pion production.

5. Vector meson production.

6. Multiple pion production.

7. Associated production and $\Delta S \neq O$ reactions.

8. $\Delta S / \Delta Q$ and $\Delta S=2$ and $T$ invariance tests.

9. Locality tests, V-A interference tests and Regge pole dominance tests.

10. $\nu_{e}$ interactions with protons.

11. Neutral current search.

12. Inclusive reaction information from all channels. 
A MEASUREMENT OF THE INTENSITY AND POLARIZATION OF MUONS PRODUCED DIRECTLY BY THE INTERACTION OF PROTONS WITH NUCLEI

R. Adair, H. Kasha, R. Kellogg (Yale Univ.); R. C. Larsen, I. Leipuner, L. Smith, H. Williams (BNL); R. Stefanski (NAI)

\section{Objectives:}

To measure the flux and polarization of prompt muons produced directly by the interactions of $300 \mathrm{GeV}$ and $400 \mathrm{GeV}$ protons with matter. The measurements will be made as a function of muon energy and transverse momentum. Such measurements will serve to:

a) Study the production of vector mesons in hadron-hadron collisions at high energy and large transverse momentum where the neutral vector mesons will be identified through their decays into muon pairs where the muons will have no net helicity.

b) Search for the production of unusual particles such as the $W$ (intermediate vector boson) and heavy leptons by examining the flux of prompt muons which have a net natural helicity (opposite to the helicity of muons from meson decays). The experiment will be sensitive to the production of such particles if they are produced with total cross sections of the order of $10^{-36} \mathrm{~cm}^{2}$.

\section{Experimental Technique:}

The proton beam will interact with a variable density copper beam-stop located at the end of the decay-pipe in the Proton Area. Directly downstream from the target a dipole magnet will select the sign of the muons and direct the beam to the detectors. A few hundred feet downstream from the target, hodoscopes will measure the transverse momenta of the muons. The muon detector is an aluminum-scintillator sandwich located at the end of the muon shield. The muon stops in the detector and decays. Measurement of the decay asymmetry of the muons establishes the polarization of the muon beam. 


\section{MASS SPECTRUM AND DECAY MODES FOR BOSONS WITH MASSES UP TO $15 \mathrm{GeV}$}

G. Blanar, C. Boyer, D. Earles, W. Faissler, D. Garelick,

B. Gottschalk, M. Jordan, E. Pothier, D. Potter, M. Ronan,

M. Tautz, E. von Goeler, J. Wang, R. Weinstein

(Northeastern University)

A "missing mass" spectrometer experiment is proposed to study the spectrum of hadrons produced in reactions

$\pi(K$ or $\mathrm{p})+\mathrm{N} \rightarrow \mathrm{N}+\mathrm{X}$ where $\mathrm{X}$ has mass $\mathrm{M}_{\mathrm{X}}$ up to $\sim 15 \mathrm{GeV}$.

This experiment makes use of a missing mass spectrometer to explore, at momentum transfer $\sim .2<-t<.4 \mathrm{GeV}^{2}$, mass spectra of hadrons up to $\mathrm{M}_{\mathrm{X}} \sim 15 \mathrm{GeV}$. At a beam momentum of $200 \mathrm{GeV}$ the resolution in mass squared is $\Delta M_{X}^{2} \sim 2 \mathrm{GeV}^{2}$. It is almost independent of $M_{x}$ and roughly scales with $s$. This phase is an ideal and sensitive (cross sections down to a few microbarns) probe for new heavy particles. It is worth noting that in the past all the well established heavy bosons, for instance, have shown up clearly in missing mass spectra. At the same time measurement of the missing mass "background" over the large range of $s$ and $M_{X}^{2}$ allows testing of current ideas about inclusive reactions. For example, in the triple Regge picture separation of the various contributions to the cross section becomes possible. 300 hours of beam time are necessary to map the complete range of $s, M_{X}^{2}$ accessible.

Summary reviewed by

E. von Goeler, February 1974 
SEARCH FOR THE INTERMEDIATE BOSON, LEPTON PAIR PRODUCTION, AND A STUDY OF DEEPLY INELASTIC REACTIONS UTIIIZING HIGH ENERGY NEUTRINO INTERACTIONS IN LIQUID NEON

C. Baltay, M. Kalelkar, J. Schmidt (Columbia); R. Palmer and N. Samios (BNL)

This experiment uses the 15' bubble chamber with (1) a hydrogen filling and a double plate for 100,000 pictures and (2) a neon filling and single plate for 900,000 pictures. A broad-band neutrino beam operating at the highest machine energy is required. Muons are distinguished from hadrons in this experiment by inserting in the chamber a stainless steel plate ( 30 tons) in which $\sim 998$ of the hadrons will interact. Since both sides of the plate are visible in the photograph, muon identification can be made on the scanning tables. It is proposed to measure the neutrino spectrum from (1) the $\pi$ and $K$ yields from the target and muon distribution in the shield and (2) from the distribution of events in the bubble chamber for reaction with known cross sections. It is expected to obtain a 58 absolute flux normalization.

The physics objectives of this experiment are:

I. The neon experiment:

1) Lepton pair production in a coulomb field.

2) W-boson decay modes.

3) Deep inelastic scattering to determine $W_{I}, W_{2}$ and $W_{3}$.

4) Total cross section measurement to $300 \mathrm{Gev}$.

5) Search for weak neutral currents.

6) Search for new particle production (charm?)

II. The hydrogen experiment:

1) $N^{*}$ events to determine the neutrino spectrum

2) Total cross section measurement.

3) Study the details of the hadronic component. 

A PROPOSAL TO MEASURE POLARIZATION IN pp, $\pi^{-} \mathrm{p}$ AND $\pi^{+} \mathrm{p}$
ELASTIC SCATTERING AT 50, 100, AND $150 \mathrm{GeV} / \mathrm{C}$

ANL, Harvard, LBL, Los Alamos, NAL, New Mexico State Univ., Wyoming, Yale 0 . Chamberlain (Spokesman)

It is proposed to measure the polarization parameter in pp, $\pi$ p and $\pi^{+} p$ elastic scattering at incident momenta of 50 . 100 , and $150 \mathrm{Gev} / \mathrm{c}$ over the range of $0.15 \leq|-t| \leq 1.5(\mathrm{GeV} / \mathrm{c})$ ?'. The apparatus consists of a polarized proton target, a double arm spectrometer which determines the angles and momenta of both outgoing particles, and on-line computer. Good angular and momentum resolution as well as high counting rate capability are achieved by the use of proportional wire chambers in both arms. The fractional momentum resolution $\Delta p / p$ is estimated to be $\pm 0.7 \%$ in the forward arm spectrometer and \pm 3.58 at large $|t|$ to \pm 68 at small $|t|$ for the recoil arm spectrometer. The apparatus is designed to make full use of the expected high beam intensities at NAL. There are no detectors used in the incident beam. A beam intensity of up to $10^{8}$ particles per pulse is expected to be utilized. Two cerenkov counters in the forward arm identify the detected particles as a $\pi, K$, or proton. The recoil arm identifies the detected particle as a proton by time of flight.

The anticipated error in the polarization parameter $P$ is $0.005 \leq \Delta P \leq 0.01$ over the range $0.15 \leq-t \leq 0.8(\mathrm{GeV} / \mathrm{c})^{2}$.

This experiment will measure a fundamental parameter, which is essential in understanding the elastic scattering of elementary particles in a new energy region. The high sensitivity of polarization phenomena to interference effects makes such measurements important in imposing constraints on theoretical models. 


\section{SURVEY OF PARTICLE PRODUCTION IN PROTON COLLISIONS AT NAL}

D. C. Carey, M. Goldberg, J. R. Johnson, D. J. Ritchie, A. Roberts, J. Sauer, R. Shafer, D. Theriot, J. K. Walker (NAL); F. E. Taylor (Northern Illinois University)

The purpose of this work is to measure the single photon inclusive cross section for the reaction $p+p \rightarrow \gamma+a n y t h i n g$ at incident proton energies from 50 to $400 \mathrm{GeV}$. The laboratory angles are from approximately 10 to $275 \mathrm{mrad}$. The experiment is in progress in the Internal Target Area using both gas jet and rotating foil targets. The basic equipment for examining the directly produced photons includes a scintillation counter for vetoing charged particles, a lead-glass total-absorption counter, and veto counters behind lead absorber for rejecting energetic muons.

Summary reviewed by

J. K. Walker, January 1976 


\section{$K^{\circ} \mathrm{P}$ AND $\bar{K}^{\circ} \mathrm{P}$ INTERACTIONS FROM $-20-60 \mathrm{GeV} / \mathrm{C}$ IN A LARGE LIQUID HYDROGEN BUBBLE CHAMBER}

H. A. Gordon, M. Habibi, K. W. Lai, P.V.C. Hough (BNL);

J. R. Albright, v. Hagopian, J. E. Lannuti (Florida State Univ);

M. S. Webster (Vanderbilt Univ.)

This proposal for the 15-ft. Hydrogen Bubble Chamber requests 106 pictures with tantalum plates at the downstream end of the chamber. The bubble chamber is to be exposed to a neutral beam created in a target $275 \mathrm{~m}$ from the chamber. The proponents request $10^{5} \pi^{-} /$pulse on this target.

The purposes of the experiment are:

1. $k_{L} p \rightarrow k_{S}^{O} p$ to investigate the difference in the elastic cross sections $\mathrm{K}^{+} \mathrm{n} \rightarrow \mathrm{K}^{+} \mathrm{n}$ and $\mathrm{K}^{-} \mathrm{n} \rightarrow \mathrm{K}^{-} \mathrm{n}$.

2. Particle and antiparticle cross-section comparison.

3. Test of one-pion-exchange in high energy by $K_{L p}^{O} \rightarrow K * \Delta$.

4. Search for high-mass $\rho$-like and $\phi$-like mesons via double Regge exchange.

5. Test of $\left(K^{*}, K^{* *}\right)$ exchange degeneracy from the process $\overline{\mathrm{K}}^{\circ}+\pi^{+} \Lambda$ and a measurement of the $\Lambda$ polarization.

6. Search for new particles. 
SEARCH FOR BARYON RESONANCES UP TO $10 \mathrm{GEV}$ MASS PRODUCED in $p+p \rightarrow p+M M$ WITH A RESOLUTION OF $\pm 25 \mathrm{MeV}$.

K. Abe, J. Alspector, K. Cohen, B. Maglich, B. Robinson, F. Sannes, (Rutgers): G. Cvijanovich, J. Oostens (Upsala College)

It is proposed to investigate the mass spectrum of nonstrange baryonic resonances of isospins $1 / 2$ and $3 / 2$ in the mass range from 4 to $10 \mathrm{GeV}$. If the emperical interval rule is valid, 84 resonances are expected in this range. The missing mass Jacoblan peak method is used, namely the kinematic region is employed where the magnitude of the missing mass is almost solely dependent on the angle of the recoil proton and relatively independent of its momentum.

In this experiment, for which no magnets are required, only the recoil proton is measured. Its angle and momentum are measured with hodoscopes by time-of-flight, and with range and pulse height analysis, (g) The resultant missing mass resolution is $\pm 25 \mathrm{MeV}$ or better. The Jacobian peak method applies to only one set of kinematical conditions, so that the momentum transfer ( $t$ value) cannot be varied or chosen freely.

The experiment is being performed in the internal proton beam using the hydrogen jet target.

A peak with a signal to background ratio of $1 / 100$ could be detected with five standard deviations in one day of running.

Summary Reviewed by F. Sannes, May 1973 


\section{ELASTIC SCATTERING OF THE LONG-LIVED HADRONS}

L. Fajardo, A.J. Slaughter, R. Majka, J. Marx, P. Nemethy,

J. Sandweiss, A. Schiz (Yale); M. Atac, P. Gollon, J. Lach,

J. MacLachlan, A. Roberts, S. Ecklund, C. Ankenbrandt,

R. Brown, G. Shen (Fermilab)

The objective of this experiment is to measure the elastic and quasi-elastic scattering of the charged long-lived hadrons on hydrogen and selected heavy nuclei in the angular region from 0.2 to 24 milliradians. In the case of pions of both signs and protons it is planned to gather more than $10^{5}$ events at several energies from $50 \mathrm{GeV}$ to $200 \mathrm{GeV}$ to give measurements of the real part of the forward scattering amplitude to \pm .01 by observation of the coulomb interference. Measurements with $K$ 's and antiprotons will give a somewhat less accurate measurement of the real part. The quasi-elastic scattering in the region of small missing mass is recorded at the same time as the elastic. The mass resolution is of the same order as the widths of the known peaks, and the measurements should allow determination of the cross sections for the production of the prominent low mass states. The data on nuclei would allow accurate measurements of the elastic scattering cross sections, made possible by the high angular resolution of the setup. It will be seen whether there is indeed a decrease in the cross section due to coherent intermediate states.

These goals require a beam of about $3 \times 10^{5}$ particles per second, with a momentum resolution of $0.05 \%$, a beam spot with a small intensity in the tail, and mass identification.

The detector consists of a high resolution $(\sim 70 \mu \mathrm{m})$ proportional chamber system to determine the scattering angle and a high resolution proportional chamber magnetic spectrometer to provide a measurement of the elasticity of the events. Data from the high resolution chambers is immediately encoded and used in the trigger tc define scattered particles. The system is capable of accepting over 800 triggers per machine pulse. 
STUDY OF LEPTON PAIRS FROM PROTON-NUCLEAR INTERACTIONS : SEARCH FOR INTERMEDIATE BOSONS AND LEE-WICK STRUCTURE

J. Appel, M. Bourquin, J.M. Gaillard, I. Gaines, D. Hom, L. Lederman, H. Paar, J.P. Repeliin, D. Snyder, J. Weiss, J. Yoh (Cl umbia); B. Brown, T. Yamanouchi (NAL)

The experiment will be performed in stages (see \#288). The first stage will be a single-arm study of $e^{ \pm}$at large $p_{\mathrm{T}}$. This is a study of the momentum continuum and of the angular distribution of single electrons arising from the reaction

$$
p+p+e^{+}+e^{-}+\text {anything. }
$$

Superimposed on the continuum there could be resonance-like bumps due to the two-body decays of massive objects, like the intermediate boson

$$
\mathrm{x}^{ \pm}+\mathrm{e}^{ \pm}+v
$$

or heavy charged vector bosons, neutral weak boson or Lee-Wick structure:

$$
x^{\circ}+e^{+}+e^{-} \text {. }
$$

This experiment is sensitive to objects in the mass range 2. 5-28 GeV.

The equipment consists of a magnetic spectrometer with counter hodoscopes $(\Delta \mathrm{p} / \mathrm{p} \sim 4 \mathrm{q})$, of an electron detector made of interleaved $\mathrm{dE} / \mathrm{dx}$ scintillation and lead glass counters followed by total absorption lead glass counters with good energy resolution ( 28$)$, by a hadron calorimeter and a muon detector.

They also look for the direct production of muons through a movable $30^{\prime \prime}$ tungsten plug.

During these runs they will accumulate information on $\pi^{\circ}$ and charged particle spectra. 
R. K. Adair and $H$. Kosha (Yale University), R. C. Larsen, I. B. Leipuner and L. S. Smith (Brookhaven National Laboratory).

\section{Summary:}

This quark search is interded to be sensitive not only to particles of charge $1 / 3 \mathrm{e}$ and $2 / 3 \mathrm{e}$ but also to particles of charge $4 / 3 e, 5 / 3 e$ and greater. The quarks are determined by their charge by measuring their ionization energy loss in a series of eight scintillation counters. The pulse heights from the eight counters are examined separately to minimize the effects of statistical fluctuations and of the Landau tail, which creates a bias toward large pulse heights. The search would be carried out in both positively and negatively charged beams at momenta determined by the primary proton beam.

With a primary energy of $500 \mathrm{GeV}$, quarks could be produced as heavy as $15 \mathrm{Gev} / \mathrm{c}^{2}$. There would be a good chance of detecting quarks if their production cross section is as great as $10^{-39} \mathrm{~cm}^{2}$. Total running time of 100 hours is estimated.

(JkW)

Received: June 1970

Approved: December 1970

Reviewed: Nay 30, 1972 


\section{A PROPOSAL TO SEARCH FOR FRACTIONALLY CHARGED QUARKS}

J. Sculli (New York University); T. Nash, T. Yamanouchi (NAL)

This is a counter experiment to search for fractionally charged quarks using one of the secondary beam channels in the Meson Area. By tuning the elements of the beam line to a momentum (for singly charged particles) higher than the primary proton beam momentum, no particle with charge $|Q|>1$ can pass through the entire length of the beam channel, unless it is scattered several times by collimator walls, magnet poles, or other elements in the beam channel. On the other hand, quarks with charge $|\mathrm{Q}|=2 / 3$ and momentum $\sim 2 / 3$ po or charge $|Q|=1 / 3$ and momentum $\sim 1 / 3$ Po, where $p_{0}$ is the tuned momentum value of the beam channel, would pass through.

An array of scintillation counters followed by an ionization calorimeter will measure the ionization loss and total energy of those particles transmitted by the channel. Further rejection against relativistic particle backgrounds will be attained by a threshold cerenkov counter.

Kinematically this experiment is sensitive for quarks with mass up to $9 \mathrm{GeV} / \mathrm{c}^{2}$. One event per hour in this experiment, assuming $2 \times 10^{12}$ interacting protons/pulse, $\Delta p / p=1 \%$, and $\Delta \Omega=.5 \mu$ ster, corresponds to

$$
\frac{d^{2} \sigma}{d p \cdot d^{\Omega}} \approx 4 \times 10^{-35} \mathrm{~cm}^{2} / \text { ster. GeV/c. }
$$

Summary sent to T. Yamanouchi

for review, May 1973 
SEARCH FOR MAGNETIC MONOPOLES PRODUCED AT FERMILAB. R. A. Carrigan, Jn. F. A. Nezrick, B. P. Strauss (Fermilab)

This experiment searches for monopoles with a rest mass up to $15 \mathrm{GeV} / \mathrm{c}^{2}$ and is capable of establishing an upper limit on the production cross section of $10^{-44} \mathrm{~cm}^{2}$. The experiment detects monopoles of charge $1 / 30$ to $24 g_{D}\left(g_{D}=e / 2 \alpha\right)$.

\section{Objective:}

Search for free monopoles produced in strong and electromagnetic interactions by using collectors placed in the proton beam dump and an electronic detection technique similar to one previously employed in cosmic ray searches.

In the experiment, an attempt is being made to find monopoles produced in Fermilab beam-stops and targets. The experimental collection technique is tailored to the kinds of beam-stops and targets available.

A monopole collector of paramagnetic or ferromagnetic materials such as aluminum or iron is inserted in the center of the interaction region of the beam stop or target. After exposure to the beam, the collector is placed at the end of an extraction solenoid. The magnetic field of the extractor overcomes the magnetic binding of the collector.

The experiment can handle proton exposures from $10^{18}$ to $5 \times 10^{18}$ protons.

An upper limit has been published for the monopole production cross seqtion of $\sigma=6 \times 10^{-42} \mathrm{~cm}^{2}$ based on an exposure of $3.4 \times 10^{16}$ protons at $300 \mathrm{GeV}$ and $\sigma=5.1 \times 10^{-42} \mathrm{~cm}^{2}$ at 400 $\mathrm{GeV}$,

We still would like a good exposure at $500 \mathrm{GeV}$ to complete the search at the highest available energy. Note that the same apparatus has been used recently to search for magnetic monopoles in air and sea water. Collectors have been exposed at the ISR for one year and will be processed shortly. 


\section{PRELIMINARY SURVEY OF $200 \mathrm{GEV}$ PROTON INTERACTIONS \\ WITH COMPLEX NUCLEI}

ANL, BNL, Univ. of Chicago, Carnegie-Mellon Univ., Purdue University, Los Alamos Scientific Laboratory, Univ. of Illinois at Chicago Circle, Orsay, Reid College

M. W. Weisfield (Spokesman)

This is a proposal for a broad program of nuclear chemistry submitted by the "Nuclear Chemistry Users Group." Three types of experiments are proposed:

1. Measurement of the absolute cross section for $\mathrm{c}^{11}$ production from $\mathrm{c}^{12}$ in a proton beam. These and other convenient foil activation studies will be useful to the calibration of beam intensities.

2. Use of mica and lexan track detectors to study multiplicities, ranges, and angular distribution of heavy fragments from proton interaction with high $z$ nuclei. These studies would determine fission cross sections.

3. A survey, by radioactivity measurements, of cross sections and momentum properties of reaction products for a few selected target elements exposed to high energy proton beams.

\section{objectives:}

1. To do a series of experiments as described above in the Neutrino and Meson Areas.

2. To establish a facility for quick access to targets and another for on line scattering chamber experiments in the high intensity beam obtainable in the Proton Area.

Status:

Some relative cross section experiments have been completed and others are now in progress as are some recoil experiments. Plans for the Proton Area facility are progressing. 
EXPERIMENTAL PROPOSAL FOR FNAL 15-FOOT BUBBLE CHAMBER ANTIPROTON-PROTON AND KAON-PROTON INTERACTIONS AT $100 \mathrm{GeV} / \mathrm{c}$

T. Kitagaki, S. Tanaka, H. Yuta, K. Abe, K. Hasegawa, A. Yamaguchi, T. Nozaki, K. Tamai, S. Noguchi, R. Kikuchi, T. Maruyama and $Y$. Unno (Tohoku University, Japan)

S. S. Pruss and J. Lach (Fermi National Accelerator Laboratory)

This is a proposal for studying $\bar{p} p$ and $K^{-} p$ interactions at $100 \mathrm{GeV} / \mathrm{c}$ using $100 \mathrm{~K}$ pictures from an exposure of the 15-foot hydrogen-filled bubble chamber to a $100 \mathrm{GeV} / \mathrm{c}$ unseparated negative beam. A beam kicker magnet is used for obtainning the clean pictures with the maximum beam tracks of 7 . About $10 K$ events of $\bar{p} p$ and $3.8 \mathrm{~K}$ events of $\mathrm{K}^{-} \mathrm{p}$ interactions are expected within the fiducial volume with using only the Gass tagging system. If the enriched beam device is used with the mass tagging system, about $34 \mathrm{~K}$ events of $\bar{p}$ interactions are expected. Physics interest resides in the following:

1. Measurement of charged multiplicity and $\pi^{\circ}$ multiplicity.

2. Inclusive studies of $v^{\circ}$ events such as $\gamma\left(\pi^{0}\right), K^{0}$, and $\Lambda_{+}^{0}$ Study of distributions of missing mass off proton, and $\Delta^{+7}$. Study of vector meson production.

3. Exclusive reactions of diffractive process including elastic scattering and $\Delta \Delta^{++}\left(\bar{\Delta}^{++}\right)$production.

4. Study of $K^{-} \mathrm{p}$ interactions similar to the $\overline{\mathrm{p}}-\mathrm{p}$ physics listed in the above. 
PROPOSAL FOR AN EXPOSURE OF THE 15-FOOT DEUTERIUM-FILLED BUBBLE CHAMBER TO A BEAM OF SEPARATED

$\pi^{+}$MESONS AT $40 \mathrm{GeV} / \mathrm{C}$ AT NAL

Purdue High Energy Physics Group

L. J. Gutay (Spokesman)

It is proposed to study the interaction of $40 \mathrm{GeV} / \mathrm{c} \pi^{+}$ in the NAL I5-foot bubble chamber filled with deuterium. A total of 600,000 pictures with a separated $\pi^{+}$beam is requested. The principal aim of the experiment is to study the production mechanism and properties in the full angular region of reactions where there are an even number of pions in the final state. These would be interactions on the bound neutron of the form

$$
\pi^{+} N+n \pi+p+p_{s}(n \text { even) }
$$

which are four constraint fits. With this sample of clean events it is proposed to study the multipion mass spectrum and production mechanisms. 
A PROPOSAL TO STUDY INELASTIC DIFFRACTIVE PROCESSES BY OBSERVING COHERENT PRODUCTION OF MULTI-PION FINAL STATES FROM He NUCLEI

T. Burnett, V. Cook, S. Csorna, L. Dunn, D. Forbush, R. Kenyon, H.J. Lubatti, A. Jonckheere, K. Moriyasu, B. Robinson, F. Rohrbach, w. Wallick (Univ. of Washington); D. Fournier, P. Heusse, P. Rancon, J.J. Veillet (Orsay)

This group proposes to study the diffractive dissociation of pions into multi-pion final states for the reaction $\pi^{-}+\mathrm{He}^{4} \rightarrow \mathrm{He}^{4}+\mathrm{X}^{-}$. Measurements of $\mathrm{d} \sigma / \mathrm{dtdm}^{2}$, the average multiplicity of charged particles and the momentum spectrum of the secondaries which are below $20 \mathrm{GeV}$ will be obtained. The t-range covered is .0l $<|\mathrm{t}|<.09(\mathrm{GeV} / \mathrm{c})^{2}$. It is planned to do this experiment at two energies, typically 100 and $200 \mathrm{GeV}$.

A streamer chamber 1 meter long by $50 \mathrm{~cm}$ wide filled with helium serves as the target as well as the detector of the recoiling helium nucleus. Measuring both momentum and range (or momentum and $\mathrm{dE} / \mathrm{dX})$, it is possible to identify the $\mathrm{He}^{4}$ in the final state and therefore assure that there are no contributions from nuclear break up.

Because $\mathrm{He}^{4}$ has $\mathrm{J}=0, \mathrm{~T}=0$, only isospin $=0, \mathrm{C}=\mathrm{G}=\mathrm{P}$ can couple. This means essentially that only the $P, P^{\prime}$, and $w$ trajectories can contribute. Including the $G$ parity constraint at the pion vertex requires that the inclusive cross section be given by $d \sigma \sim\left|P+P^{\prime}\right|^{2}+|w|^{2}$. In the triple Regge limit, there are a maximum of eight triple Regge couplings including all cross terms (to be compared with the more than one hundred terms obtained in pp inclusive when one includes $P, P^{\prime}, \omega, \pi, \rho, A$ trajectories). This experiment is thus in a good position to check the predictions of the various diffractive and triple Regge models.

More generally, a study will be made of the dependence of these nearly pure diffractive cross sections as a function of center-of-mass energy, momentum transfer, produced mass, and charge multiplicity. 
SEARCH FOR HEAVY LEPTONS AND INTERMEDIATE BOSONS FROM PHOTON-NUCLEON AND PHOTON-NUCLEI COLLISIONS

J. Bronstein, R. Coleman, L. Cornell, G. Gladding, M. Gormley, R. Messner, T. O'Halloran, J. Sarracino, A. Wattenberg, D. Wheeler (Univ. Of Illinois); M. Binkley, I. Gaines, R. Orr, J. Peoples, A.L. Read (Fermilab); B. Knapp, W. Lee, P. Leung, S. Smith, A. Wijanco (Columbia); J. Knauer, D. Yount (Univ. of hawaii); D. Nease (Cornell)

This is a counter (and proportional chamber) experiment using a high-energy, high-intensity, untagged photon beam. The objectives of this experiment are:

1. To determine the existence or non-extence of particles such as the heavy lepton ( $l$ ) and intermediate vector boson $(W)$, in 1 - $10 \mathrm{GeV} / \mathrm{c}^{2}$ mass range. Since the coupling of the photon to charged leptons is well-known, one can calculate the absolute rate and the angular distribution for the production of charged particle pairs of any mass. If the intermediate vector boson exists in this mass range, this experiment will provide a unique way of determining its anomalous magnetic moment from the observed production cross section.

2. To explore the mass range from 1 to $10 \mathrm{GeV} / \mathrm{c}^{2}$ for the presence of $\mathrm{JP}^{\mathrm{P}}=\mathrm{I}^{-}$states. Since the photon is coupled to the $\mathrm{J}^{\mathrm{P}}=1^{-}$ family of particles, the use of a high energy photon beam provides a natural mechanism for the diffractive production of such states. The detector can identify and analyze these states through their leptonic decay modes, multihadron decay modes or mixed decay modes involving the $\psi(3.1)$.

3. To measure the cross section of vector meson photoproduction. The $p(765)+2 \pi$ and $\rho^{\prime}(1000)+4 \pi$ states have been observed in sufficient number to measure their cross sections. The photoproduction cross section of the $\psi(3.1) \rightarrow \mu \mu$ has been measured in the momentum interval of $60 \mathrm{GeV} / \mathrm{c}$ to $220 \mathrm{GeV} / \mathrm{c}$ to $30 \%$, while the photoproduction $\psi(3.7)$ has been observed through the decay into $\psi(3.1)+2 \pi$. Additional measurements will be made to extend the energy domain and $t$ domain of these cross sections.

4. The observation of the $\psi(3.1)$ and $\psi(3.7)$ have raised the question of the existence of charmed particles and colored particles. A search for the former will be carried out as a by-product of I tem 3. by looking for diffractive production of a charm - anti charm meson pairs. Decays which involve $\mathrm{K}_{\mathrm{S}} \rightarrow \pi^{+} \pi^{-}$and leptonic decays are the most sensitive. The latter question will be examined by looking for $\pi^{+} \pi^{-} \gamma$ and $\pi^{+} \pi^{-} \pi^{+} \pi^{-} \gamma$ decay modes of $\psi(3.1), \psi(3.7)$ and of course entirely new states. Again the search is a by-product of Item 3 . A sensitivity of branching ratio times cross section of $2 \times 10^{-34} \mathrm{~cm}^{2}$ has been demonstrated for $300 \mathrm{GeV} / \mathrm{c}$ protons. The sensitivity improves markedly at higher proton energies. 


\section{INTERACTIONS OF $150 \mathrm{GeV} \pi^{-}$MESONS IN A LARGE NAL BUBBLE CHAMBER FILLED WITH $\mathrm{H}_{2}$ - $\mathrm{Ne}$}

H. Bingham, W. Fretter (University of California, Berkeley)

It is proposed to study $\pi^{-}$interactions in a mixture of $\mathrm{H}_{2}$ and neon ( $15 \% \mathrm{Ne}$ ) in the NAL 15-foot bubble chamber. A $\pi$ beam of the highest possible energy is requested in an exposure of 250,000 pictures with 3-4 beam tracks per picture. The riajor thrust of this experiment is to determine cross sections for diffractive production of three-pion, five-pion and possibly seven-pion systems by negative pions on neon nuclei. They will also study events where neutral pions are produced, both coherently and in interactions with protons in the liquid, and will use the $\gamma$-veto capabilities of the chamber to reduce the background in $4 \mathrm{C}$ fits on protons such as $\pi^{-} p+\pi^{+} \pi^{-} \pi^{-} p$. They will search for quarks among the beam particles and for other exotic states produced in nuclear cascades.

Summary reviewed by

W.B. Fretter, February 1976 


\title{
CRACOW NUCLEAR EMULSION EXPOSURES
}

\author{
J. Gierula \\ (Institute of Nuclear Reseaxch, Cracow, Poland)
}

Summary:

This proposal requests a nuclear emulsion exposure with protons and pions.
a. Beam:
Protons, $500 \mathrm{GeV}$
Pions, same energy as protons
b. Intensity: $1-3 \times 10^{5}$ particles/cm ${ }^{2}$.
c. Emulsion: Ilford G-5, 15 sheets each $600 \mu$ thick and $7.5 \mathrm{~cm} \times 15 \mathrm{~cm}$. Beam parallel to the $15 \mathrm{~cm}$ side.

a. Alignment: Better than \pm 10 millirad.

This group has obtained an exposure of emulsions to $60 \mathrm{GeV} / \mathrm{C} \pi$ at Serpukhov. Results presented at lund conference. Their primary interest is in jets.

Emulsion Processing: Perhaps ANL

Scanning: Cracow

Received: June 23, 1970

(JKH)

Approved: $\quad 8-70$ 


\title{
PROPOSAL FOR EXAMINATION OF WIDE ANGLE GAMMA RAYS ENERGY
}

\author{
MASS SPECTRA AND CHARGED PARTICLE CORRELATIONS AT FERMILAB
}

M. Binkley, B. Cox, T. Kondo, T. Murphy, T. Toohig (Fermilab); C. Y. Chien, L. Madansky, J. Nagy, A. Pevsner (Johns Hopkins University)

This experiment will initially measure the mass spectrum, dynamics, and both the coplanar and non-coplanar cross sections of two or more photon final states in the reactions

$$
\begin{aligned}
p p & \rightarrow \gamma \gamma+x \\
\text { and } & p p \rightarrow n \gamma+x
\end{aligned}
$$

using the high intensity proton beam available in the P-West section of the Proton Area at energies between 100 and $500 \mathrm{GeV}$ incident on $\mathrm{H}_{2}$ and different $A$ foils. Concurrently with the multiphoton phase of the experiment we will take data on single photon energy and angular distributions at a wide variety of angles. In the photon experiments we hope to investigate the pseudoscaler mass spectrum of two or more photons (with 5\% resolution) to investigate processes such as resonance formation (analogous to the vector $\psi$ production) or parton annihilation cross sections such as have been calculated by Paschos which lead to massive gamma pairs or $\pi^{\circ}$ f final states. Correlation cross sections of $10^{-33}$ $\mathrm{cm}^{2}$ are readily accessible to the experiment.

In addition, some charge particle-photon work will be done in the two arm spectrometer since momentum analysis and lepton $(e, m)$ identification can be done on the charged particle spectra present in the pp interactions in some kinematic regions. We are also to study the correlations both between charged particles and charged particles and photons.

We are currently performing a final installation of apparatus on the two arm spectrometer under construction in the P-West 50 foot pit. This includes two $\mathrm{Pb}$ glass arrays totaling fifty $24 \mathrm{r.1}$. counters, 65 lucite cerenkov and scintillator counters in various hodoscopes for charged particle and photon shower identifications, and 20 planes of proportional chambers totaling 4000 wires. Two 6-inch aperture magnets have been installed and the final shielding configuration is finished. We have made four preliminary runs to determine optimal proton beam configurations and modifications that have been made to correct halo problems, and one extensive data and calibration run in September was done october 1975. We will require a minimum of 100 hours of data taking at each energy for the photon work.

Summary Prepared By: B. Cox 
FOCUSING SPECTROMETER FACILITY

ANL, Bari, Brown, CERN, Cornell, MIT, NAL, Northeastern, Stanford D.M. Ritson (Spokesman)

This group has constructed a focusing spectrometer at the end of the $2.5 \mathrm{mrad}$ beam. The solid angle acceptance is variable from $15 \mu$ ster at $60 \mathrm{GeV} / \mathrm{c}$ to $5 \mu \mathrm{ster}$ at $200 \mathrm{GeV} / \mathrm{c}$. Momentum resolution is $\pm .1 \%$ at present and should improve. By suitable beam cptics techniques, it will be possible to operate the overall syster. in either an achromatic or energy loss mode. Changes in scattering angle are accomplished by steering some combination of the incident and outgoing particles. Particle identification is achieved with differential and threshold Cerenkov counters in both the beam and spectrometer. Proportional planes located at suitable points enable the experimenter to decode outgoing particle variables such as momentum and production angle. The estimate for programmed data taking is 1000 hours.

Major Physics Objectives:

1. Measure elastic scattering for the reactions of protons, antiprotons, positive and negative pions, and kaons on hydrogen in the region $0 \leq|\mathrm{t}| \leq 1.5 \mathrm{GeV} / \mathrm{c}^{2}$ and $50 \leq P_{\text {inc }}$ $\leq 200 \mathrm{GeV} / \mathrm{c}$ to obtain Regge $\alpha$ and $\alpha^{\prime}$ coefficients, crossovers, and overall behavior to a few percent.

2. Measure inelastic scattering in the same region for masses up to about $4 \mathrm{GeV}$ to cover the resonance and triple-regge region to about 58 .

3. Repeat the measurements with a deuterium target.

Summary prepared by

D.M. Ritson, March 1974 
C. Ankenbrandt, M. Atac, P. Gollon, J. Lach, J. MacLachlan,

A. Roberts, G. Shen (Fermilab); L. Fajardo, R. Majka, J. Marx, P. Nemethy, J. Sandweiss, A. Schiz, W. Willis (Yale).

This experiment proposes to measure small angle scattering of most of the hyperois. The $|t|$ range is between 0.1 and $0.6 \mathrm{GeV} / \mathrm{c}^{2}$. As part of the proposed program, production cross sections in the forward direction of the charged hyperons, $\Sigma_{-}^{-} \Xi^{-}$and $\Omega^{-}$, will be measured. Positive hyperons $\Sigma^{+}, \bar{L}^{+}, \bar{\Sigma}^{+}$and $\bar{\Omega}^{+}$will be produced along with a substantial proton flux. It is felt that cross section measurements are possible for those produced with appreciable cross sections. As a by product we would also measure their total cross section. Particle detection and identification will be done with two methods. One uses a high resolution gas cerenkov counter and the second relies on observing the hyperon decay products.

A second goal of this experiment its to search for new short lived exotic particles of lifetime $10^{-11}-10^{-10} \mathrm{sec}$.

One of the early engineering goals of the experiment will be the study of hyperon production cross sections as a function of hyperon momentum. This will determine at what momenta the experiment can be run most effectively.

An updated description of the detectors and layout is given in Proposal 353 which uses most of the same apparatus.

Two hundred running hours were requested in the original proposal. This should be considered as the time needed for only the first phase. 
MUON-PROTON INELASTIC SCATTERING EXPERIHENT AT THE FERMI NATIONAL ACCELERATOR LABORATORY

University of Chicago, Harvard University, University of Illinois, University of Oxford H.L. Anderson (Spokesman)

This is an experiment to study muon-proton (and muon-deuteron) inelastic scattering in the energy range of 100 to $225 \mathrm{GeV}$. Initially, the experiment will be performed with muons of energy $150 \mathrm{GeV}$. Measurements will be made with the momentum transfer squared, $q^{2}$, in the range of 0.01 to $30(\mathrm{GeV} / \mathrm{C})^{2}$ and the energy loss of the muon, $\nu$, in the range of 10 to $140 \mathrm{GeV}$. A large aperture magnetic spectrometer, which consists of the Chicago Cyclotron magnet, proportional wire chambers, and wire spark chambers, will detect the scattered muons and charged hadrons produced in the collision.

After the initial run of 400 hours at $150 \mathrm{GeV}$ with hydrogen, they propose to run for 200 hours at $100 \mathrm{GeV}$ and 400 hours at $225 \mathrm{GeV}$, then repeat these runs with deuterium.

The following information can be obtained:

1. The inelastic form factors $W_{1}\left(q^{2}, v\right)$ and $W_{2}\left(q^{2}, v\right)$ of the proton and the neutron;

2. Cross-sections in the photoproduction 1 imit;

3. The multiplicity and momentum spectra of the forward charged hadrons;

4. Partial information on the multiplicity of neutral pions; and

5. Measurement of some specific channels, e.g. $\rho$ and $\phi$ production. The cross-section as a function of $q^{2}$ and $v, t$ dependence to recoil proton, and dependence on azimuthal angle wi 71 be studied.

Suminary reviewed by

Herbert L. Anderson, January 1975 


\section{STUDY OF $\pi^{+} \mathrm{p} \rightarrow \mathrm{K}^{+} \Sigma^{+}$AND $\pi^{+} \mathrm{p} \rightarrow \mathrm{K}^{+} \mathrm{Y}^{+}$ USING THE FOCUSING SPECTROMETER FACILITY}

D. S. Ayres, D. Cohen, R. Diebold, S. L. Kramer, A. B. Wicklund (ANL); A. F. Greene, J. E. Elias (FNAL); R. L. Anderson, D. Gustavson, K. Rich, D. M. Ritson (Stanford University and SLAC); R. Prepost (University of Wisconsin)

It is proposed to use the Fermilab Single Arm Spectrometer facility to detect $\mathrm{K}^{+}$mesons produced in association with $\Sigma^{+}, \mathrm{Y}^{* *}(1385)$ and $\mathrm{Y}^{*}(1670)$ by $\pi^{+} p$ interactions. The study of these reactions is one of the easiest ways to obtain data at Fermilab on nondiffractive processes. The $s$ and $t$ dependence will give valuable information on $\mathrm{K}^{*}$ exchange as well as the importance of cuts at high energies.

These reactions will be studied at five incident momenta from 30 to $200 \mathrm{GeV} / \mathrm{c}$ and from $t_{\min }$ to about $0.8 \mathrm{GeV}^{2}$. The recoil baryon will be identified by the missing mass technique; a resolution at the $\Sigma$ mass of typically $\pm 50 \mathrm{MeV}$ is expected. The four Cerenkov counters in the beam plus four more in the spectrometer will be used to achieve the required particle identification.

The line-reversed reactions such as $\mathrm{K}^{-} \mathrm{p} \rightarrow \pi^{-} \Sigma^{+}$will also be studied at one or two momenta.

Several thousand $\mathrm{K}^{+} \Sigma^{+}$events are expected per momentum, and a total of 500 hours of running time is approved. 


\section{A PROPOSAL TO STUDY PARTICLE PRODUCTION AT HIGH TRANSVERSE MOMEINTA}

J. Cronin, H. Frisch, 14. Shocket (Univ. of Chicago); P. Piroué,

J. Boymond, R. Sumner (Princeton Univ.)

This proposal is for an exploratory search of the particle constituents at about 90 degrees in the c.m.s. of proton-proton collisions of $200-500 \mathrm{GeV}$ incicent energy $(80 \mathrm{mrad}$ in the $1 \mathrm{ab}$.$) .$

This data would provide information on:

a) Hadron production at high transverse momentum

b) The possible existence of the intermediate boson, heavy photons and heavy leptons by searching for leptons with high transverse momentum. Sensitivity to a $6 \mathrm{GeV}$ massive $W$-meson is sucin tilat lepton yield $\geq 10^{34} \sigma_{\text {B }}$ B/hour $\left(\sigma_{\text {- }}\right.$-prodiuction cross section,

c) The possible existence of long-lived particles (with or without fractional charge).

d) With snall modifications to the apparatus, shortlived particles anō also direct photon prociuction.

Required: Beam line capable of analyzing particles up to 200 $\mathrm{GeV} / \mathrm{C}$ at $80 \mathrm{mirad}$ from a one interaction length target. The beam line which is designed in the proposal has main ring quads and dipoles. The solid angle of acceptance is $16 \mu$ ster and the momentum acceptance is $10 \%$. Two independent measurements of momentum are made in the beam line. Due to the use of a focusing beam line, the counters are small and can be well shielcied. 


\section{INTRA NLCLEAR CASCADE PRODUCED BY $200 \mathrm{GEV}$ PROTONS}

\section{T. King, et.al. (University of Tennessee)}

\section{Sumnary:}

This proposal requests a nuclear emulsion exposure with $200 \mathrm{GeV}$ protons.
a. Beam:
Protons, $200 \mathrm{GeV}$
b. Intensity $5.0 \times 10^{4} / \mathrm{cm}^{2}$
c. Emulsion: Small stack of Ilford $\mathrm{K5}, 6001 \mathrm{~s}$ thick
d. Alignment: Parallel

These data would, through measurements of the angular distribution of the secondaries, provide information on the extent to which the incident proton interacted with either a single nucleon of a group of several nucleons in the nucleus. Secondly, the angular distributions will be measured as a function of multiplicity.

\footnotetext{
Emulsion Processing: U.T.

Scanning: U.T.

(JKW)

Received: December 21, 1970

Approved: February 1971

Exposure: Sept. 1972
} 


\section{MEASUREMENT OF TOTAL CROSS SECTIONS ON HYDROGEN AND DEUTERIUM}

R.L. Cool, O.D. Fackler (Rockefeller Univ.); A.S. Carroll, I-H Chiang, T.F. KYcia, K.K. Li, P.O. Mazur, D.C. Rahm (BNU); W.F. Baker, D.P. Eartly, P.F. Koehler, R. Rubenstein, A. Wehmann (NAL); P.M. Mockett (Univ. of Washington); K.F. Pretzl (Max Planck Inst., Munich); G. Giacomelli (Univ. of Padua)

It is proposed to measure total cross sections of $\pi^{ \pm}, K^{ \pm}$, $p$ and $p$ on hydrogen and deuterium between $20 \mathrm{GeV}$ and the maximum energy available at NAL. The apparatus consists of three Cerenkov counters (two differential and one threshold), three targets (one hydrogen, one deuterium and one dummy), a proportional wire chamber telescope in the incident beam, a set of transmission counters (scintillation counters) and proportional wire chambers downstream of the target and an on-line computer. The apparatus is designed to be able to measure the energy dependence of a given cross section over the full range with a relative accuracy of about 0.18 . The anticipated error in the absolute cross sections on hydrogen and deuterium is 0.38-0.58. This requires the absolute density of hydrogen and deuterium to be known to better than 0.18 .

The measurement of the total cross sections will play a basic role in the understanding of strong interactions in the new energy range. An absolute accuracy of a few tenths of one per cent will yield the energy dependence of cross sections, comparisons of cross sections within SU(3) supermultiplets, and stringent tests of high energy limiting theorems. The recent result from the CERN ISR that the p-p total cross section increases with energy emphasizes the importance of accurate total cross section measurements at NAL energies for all of the available strongly interacting hadrons. 


\title{
A PROPOSAL TO STUDY SOME CHARACTERISTICS OF PROTON NUCLEON
}

\section{AND PROTON NUCLEUS COLLISIONS AT 400 GeV USING NUCLEAR EMULSIONS}

\section{R. R. Daniel, et.al. (Tata Institute of Fundamental Research)}

\section{Summary:}

This proposal requests a nuclear emulsion exposure at the highest proton energy (200-500 GeV) possible.
a. Beam: Protons, 200-500 GeV; diameter of beam $25 \mathrm{~cm}$
b. Intensity: $(1+2,-0.5) \times 10^{4}$ protons $/ \mathrm{cm}^{2}$
c. Emulsion: 80 pellicles, 40 of normal 65
40 of hypersensitive $\mathbf{G 5}$
Each pellicle is $15 \mathrm{~cm} \times 12 \mathrm{~cm} \times 0.06 \mathrm{~cm}$ Beam is parallel to $15 \mathrm{~cm}$ edge
a. Alignment: Better than 6 milliradians

These data would:
a. Determine multiplicity in proton nucleus collisions.
b. Determine $\mathrm{K}^{+}+\mathrm{K}^{-} / \pi^{+}+\pi^{-}$xatio by measurements in the backward hemisphere in the c.m.s.
c. Determine characteristic transverse momenta of particles by measurements in backward hemisphere.
d. Search for quarks using hypersensitive emulsion.
e. Angular distributions of secondaries.
f. Interaction mean free paths.

\begin{abstract}
Emulsion processing: Tata Institute
Scanning: Tata Institute
\end{abstract}

Received: January 14, 1971

(JKW)

Approved: $4-71$ 


\section{A BEAM DUMP EXPERIMENT}

M. Awschalom, S. Baker, I. Coulson, P. Gollon, C. Moore A. Vanginneken (Fermilab)

This is a proposal to perform several related experiments whose over-all purpose is to obtain data relevant to the design of experimental devices and facilities from the point of view of radiation effects.

The investigation includes:

1. Spatial distribution of the density of energy deposition in beam dumps using various detectors.

2. Spatial distribution of radioactivation of various materials such as steel, soil, water, etc. in media such as steel and concrete.

3. Hadron cascade development in beam dumps by foil activation, scintillation counters and other detectors.

4. Undifferentiated total hadron yields at large angles by foil activation and other detectors.

5. Muon production and attenuation characteristics behind beam dumps, using Cerenkov and scintillation counters.

6. Measurements of some absolute cross sections needed for beam intensity monitoring to an accuracy of $\pm 5 \%$ or better.

A total beam time of 120 hours of good beam is requested. This is to be split between 200 and $400 \mathrm{GeV}$ operation. About 2 to 3 hours of beam time per week appears optimal. Beam intensities in the range 105 to $10^{12}$ protons per second are requested with the beam size about $3 \mathrm{~mm}$ diameter or 1 ess. 


\section{PROPOSAL TO STUDY MULTIPARTICLE PERIPHERAL PHYSICS AT NAL}

C. Bromberg, G. Fox, R. Gomez, J. Pine (Caltech); V. Ashford, R. Juhala, E. Malamud, S. Mori (NAL); E. Lorenz, P. Schlein, (UCLA); R. Abrams, H. Goldberg, S. Margulies, D. MCLeod, $\mathrm{J}$. Solomon (Univ. of Illinois, Chicago Circle); A. Dzierba, (Univ. of Indiana)

A magnetic spectrometer is proposed, to measure forwardgoing hadronic systems at 50 to $200 \mathrm{GeV} / \mathrm{c}$. The particle detectors are wire chambers, proportional counters, and threshhold Cerenkov counters. While momentum measurements are made only on forwardgoing particles, the remainder of the $4 \pi$ solid angle around the hydrogen target is covered by charged particle and gamma ray detectors.

Specific reactions will be isolated in order to study the $s$ and $t$ dependences of the cross sections for peripheral processes, search for new resonant states, measure $\pi-\pi$ and $\mathrm{k}-\pi$ elastic and inelastic scattering, and study the properties of high multiplicity events. The processes may be divided into two types, differing according to whether there is or is not charge exchange to the recoil nucleon. The non-charge exchange reaction events (e.g. proton recoils) further subdivide into two groups depending upon whether or not they are dominated by diffraction scattering. With the aid of signals from multiwire proportional chambers and other detectors various triggers will be defined to study a great variety of processes simultaneously. By computer control, each trigger will be limited to a preset maximum fraction of the "live time."

The initial proposal calls for a physics program for the spectrometer which is initially limited to those processes easiest to measure (e.g., processes where no neutral pions are produced), which nevertheless spans a large range of strong interaction problems. Priority of operation of the facility by the proposing group for 15 to 18 months is requested. After this time, the setup will be available for use by others.

Summary reviewed by

D. McLeod, January 1976 
PROPOSAL TO STUDY $\pi^{-} p+\pi^{\circ} n$ AND $\pi^{-} p \rightarrow \eta n$ AT HIGH ENERGY

A. Barnes, J. Mellema, A. Tollestrup, R. Walker (Caltech);

O. Dahl, R. Johnson, R. Kenney, M. Pripstein (LBL)

The asymptotic behavior of hadronic cross sections is one of the most important questions that NAL may be able to answer. Experiment \#11l is at present measuring the $\pi p$ charge exchange cross section up to the highest energies available in the Meson Area.

By using isotopic spin conservation and the optical theorem, an upper limit on the difference between the total $\pi p$ and $\pi^{+} p$ cross section can be established.

This cross section is also an excellent test of Regge theory since only the $p$ meson is exchanged. In addition, when the results of this experiment are combined with those of the total cross section measurements for $\pi^{+} p$ and $\pi^{-} p$, it will be possible to separate the amplitude in the forward direction into its real and imaginary parts and the consistency for the experiment with dispersion relations can be checked.

The experiment utilizes an elegant new detector that is composed of 140 narrow finger counters that locate a shower position to about $1 \mathrm{~mm}$ and integrate its total energy loss. This knowledge allows one to uniquely solve for the direction of a neutral particle that decays into $\gamma$-rays and to determine its mass squared. preliminary running has shown peaks for the $\pi^{0}+2 \gamma, n^{0}+2 \gamma, n^{0}+3 \pi^{0}, \omega^{0}+\pi^{0} \gamma$, and a large bump at the mass of the $f$.

The experiment at present has finished running at $100 \mathrm{GeV}$. It is planned that the following energies $20,40,65,100,150$, and 250 will be measured. The lower end will tie into existing measurements and should cover a range in $t$ between 0 and 1.5 $(\mathrm{GeV} / \mathrm{c})^{2}$. At the highest energy, due to the rapidly falling cross section, probably only data for $t$ less than 0.6 will be obtained with a beam of $10^{6} \pi^{-1}$ 's per pulse on the target.

A11 of the necessary equipment is now assembled and running and no additional extensive construction will be necessary. It is hoped that the energies below $100 \mathrm{GeV}$ will be finished in the current running period (through April, 1974). 
PRIMARY INTERACTIONS, MULTIPLICITY AND CROSS-

SECTION AS $t$ ( ( $\mathrm{P}$ ) FROM $200-500 \mathrm{GeV} / \mathrm{C}$.

$$
\text { P. L. Jain (SUNY at Buffalo) }
$$

\section{Summary:}

A proposal to make an unspecified number of exposures of Ilford G-5 emulsions to protons in the 200-500 Gev range. The object of the experiment is to study secondary particle multiplicities and the cross-section as a function of energy.

Beam density requirements are $10^{4}-10^{5}$ protons $/ \mathrm{cm}^{2}$.

Received: February 24, 1971

(TET)

Approved: March 6, 1972 


\begin{abstract}
A SEARCH FOR MASSIVE, LONG-LIVED, FRACTIONALLY CHARGED PARTICLES PRODUCED BY $300 \mathrm{GEV}$ PROTONS
\end{abstract}

Cal Tech Neutrino Group, M.L. Stevenson (LEL)

A search has been made for long-lived (25 $\mu \mathrm{sec}<\tau<1 \mathrm{msec})$ weakly interacting particles of rest mass greater than $5 \mathrm{GeV} / \mathrm{c}^{2}$ that come to rest and subsequently decay in the calorimeter portion of the CAL Tech-NAL neutrino detector. The 253 meters of steel, 3 meters of Al, and 178 meters of earth in front of the detector allow oniy fractionally charged particles of $2 / 3 \mathrm{e}(1 / 3 \mathrm{e})$ of moments between $184 \mathrm{GeV} / \mathrm{c}$ and $190 \mathrm{GeV} / \mathrm{c}(46 \mathrm{I} \mathrm{GeV} / \mathrm{c}$ and $47.5 \mathrm{GeV} / \mathrm{c}$ ) to stop. iro such particles wepe observed during a six-hour parasitic run, during which time $1.39 \times 10^{16} 300 \mathrm{GeV}$ protons struck the target of the "Single Horn" neutrino beam. The "90\% confidence level" upper limit to the invariant differential cross section $\mathrm{E} \frac{\mathrm{d}^{3} 0}{\mathrm{dp}^{3}}\left(0^{\circ}\right)$

times branching ratio into $5 \mathrm{GeV}$ of calorimeter energy is about $5 \times 10^{-37}$ $\mathrm{cm}^{2}(\mathrm{GeV} / \mathrm{c})^{-2}\left(4 \times 10^{-38} \mathrm{~cm}^{2}(\mathrm{GeV} / \mathrm{c})^{-2}\right)$ for lifetimes near $100 \mathrm{usec}$. 
MEASUREMENT OF TOTAL CROSS SECTIONS, MULTIPLICITY, ANGLLAR DISTRIBUTION AND CASCADE DEVELOPMENT

J. Hebert (University of Ottawa)

\section{Summary:}

A proposal to expose stacks of emulsion loaded with $\mathrm{B}^{10}$ and Lif to protons of energy $100 \mathrm{GeV}, 150 \mathrm{GeV}, 200 \mathrm{GeV}$, and $400 \mathrm{GeV}$. The purpose of the experiment to study the total cross-section for protons on complex nuclei as a function of energy; the charged multiplicity distribution as a function of energy; the angular distributions in p-nucleus collisions, and to gather statistical information on hadron shower development.

The beam density requirements are $10^{6}$ protons/pulse over a $10 \mathrm{~cm} \times 5 \mathrm{~cm}$ area with $\sim 5 \mathrm{~min}$. exposure at each energy.

Received: March 1, 1971

Approved: April, 1971 (TET) 
PHENOMENOLOGICAL STUDY OF PROTON INTERACTIONS AT $200 \mathrm{GeV} / \mathrm{C}$ IN

\section{EMULSION}

O.Kusumoto, S.Ozaki and M.Teranaka (Osaka City University);

G.Fujioka, H.Fukushima, K.Kobayakawa, M.Miyagaki and Y.Tsuzuki

(Kobe university); H.Okabe and J.Yokota (Science Education Institute of Osaka Prefecture); M.Ohta (kinki University).

\section{Summary:}

The purpose of the experiment is to study the general features of the proton-nucleon and proton-nucleus interactions at $200 \mathrm{GeV} / \mathrm{c}$ in emulsion. The cross sections, the contribution of diffractive interactions, the character of multiplicity distributions and the behavior of backward produced particles are mainly examined. The short-lived new particles are also investigated near the interaction point.

Procedure:

A stack of $\mathrm{K} 5$ pellicles of $3 " \mathrm{x} 6 \mathrm{~N} \times 600 \mathrm{\mu m}, 36$ sheets is exposed to the $200 \mathrm{GeV} / \mathrm{c}$ proton beam with a density of $10^{4} \sim 10^{5}$ per $\mathrm{cm}^{2}$. Several sandwich stacks each of which is made by three K5 pellicles of $5 \mathrm{~cm} \times 5 \mathrm{~cm} \times 600 \mathrm{~mm}$ and one metal foil (C, Al, $\mathrm{Ni}, \mathrm{Ag}$ or $W$ ) of $200 \sim 400 \mu \mathrm{m}$ thick are exposed yertically to the emulsion plane with a beam density of $\sim 5 \times 10^{4}$ per $\mathrm{cm}^{2}$. 


\section{HADRON SPECTRA FROM HIGH ENERGY INTERACTIONS}

Bari University: C. DeMarzo, L. Guerriero, P. La Vopa, G. Maggi,

F. Posa, G. Selvaggi, P. Spinelli, F. Waldner; Brown University:

D. Cutts, R. Lanou, J. Massimo; Fermilab: A. Brenner, D. Carey, J. Elias,

G. Mikenberg; M.I.T.: W. Aitkenhead, D. Barton, G. Brandenburg,

W. Busza, A. Dilmanian, J. Friedman, P. Garbincius, H. Kendali,

T. Lyons, B. Nelson, L. Rosenson, W. Toy, R. Verdier, C. Young

It is proposed to study inclusive reactions of the type $a+p($ or $n)+b+$ anything where $a=$ proton, antiproton, $\pi^{+}, \pi^{-}$, $\mathrm{K}^{+}, \mathrm{K}^{-}$, and $\mathrm{b}=$ any of the above particles and is the detected particle.

The over-all objective is to map out tise general behavior of these reactions over as wide a range of kinematic variables as possible, concentrating on those reactions which cannot be measured at the ISR. In particular, incident momenta of 50, 100, and $175 \mathrm{GeV} / \mathrm{c}$ would be used and the spectra of particle $b$ would be measured from the maximum momenta down to about $10 \mathrm{GeV} / \mathrm{c}$ in coarse steps. The range of transverse momenta covered will be determined primarily by counting rate but generally $21.5 \mathrm{GeV} / \mathrm{c}$. It is proposed to use the Fermilab focusirg spectrometer. Statistical errors will range from about $1.5 \%$ at small transverse momenta to $10 \%$ at large transverse momenta.

The target will be surrounded with detectors, the purpose of which is to study the correlation of the inclusive cross sections with charged particle multiplicities. The set-up will provide this information simultaneously with the measurements of the inclusive cross sections.

This experiment will provide a study of the approach to scaling of a number of different reactions, and will also provide information about the associated multiplicity as a function of the kinematics $\left(M^{2}, t, x\right)$ of the leading particle in these reactions. The multiplicity information can provide a very stringent test of factorization.

A DISC counter, a differential and threshold cerenkov counter will be used in the beam and three threshold cerenkov counters and a differential cerenkov counter in the spectrometer. This will permit the simultareous measurements of different reactions.

Running time is estinated at 1000 hours with 200 hours of test time in adaition. 


\section{EARLY $\pi^{\circ}$ PARTICLE PRODUCTION SURVEY WITH}

THE GAS JET TARGET

$$
\begin{aligned}
& \text { C. Rubbia, J. Pilcher (Harvard) } \\
& \text { D. Cline, R. Imlay (Univ. of Wisconsin) }
\end{aligned}
$$

\section{Summary:}

The proposal is to use lead glass counters to study the gamma ray spectrum at several angles produced from the gas jet target. The proposed angular range for detection of gamma rays is from 5 to $20 \mathrm{mrads}$. The physics objectives are:

a) Obtain the $\pi^{\circ}$ spectrum to determine the low energy neutrino yield.

b) Study anomalous large transverse momentum gamma rays which might result from $W$ meson production.

c) Study the yield of gamma rays emerging from downstream of the target to obtain the yield of $\mathrm{K}_{\mathrm{S}}$ mesons or any heavy particle that lives $10^{-10}$ secs or Ionger.

It is estimated that several days of running, with $10^{9}$ interactions in the gas jet per pulse are required.

(JKW)

Received: March 1971

Approved: June 1971

Summary Revised: May 30, 1972 


\section{A PROPOSAL TO SEARCH FOR VERY HEAVY STRANGE PARTICLES USING A SMALL HYDROGEN BUBBLE CHAMBER}

M. Alston-Garnjost (Lawrence Berkeley Lab); J. Friedman (Stanford Linear Accelerator Center); K. Hayes, W. Ko, R. Smith, R. Sommer, R. Lander, D. Pellett, P. Yager, J. Erwin, D. Faulkner (Univ. of California, Davis)

This is a revision to the original proposal after approval of $50 \mathrm{~K}$ pictures of $\pi^{+} \mathrm{p}$. It is suggested that a large bare chamber run in the 30 " bubble chamber could give important results, namely the confirmation or refutation of the isotropic and"black body" nature of meson production.

They have found at energies below $28 \mathrm{GeV}$ that the low energy pions or kaons are produced quite isotropically in the center of mass and that a "black body" boson distribution with a "temperature" of $140 \mathrm{MeV}$ (KT) fit the data quite well. If this persists at high energy it would violate all scaling models and so require a non-scaling component.

Summary sent to $\mathrm{R}$. Lander for review, May 1973 


$$
-60-
$$

PROPOSAL TO STUDY $\pi$ P REACTIONS at 60 and $200 \mathrm{GeV} / \mathrm{c}$

IN THE 30-INCH HYDROGEN BUBBLE CHAMBER AT NAL

V. T. Cocconi, T. Coghen, M. Counihan, U. Gensch, J. D. Hansen, G. T. Jones, V. Karimaki, G. Kellner, W. Kittel, A. Kotanski, D. Kuhn, D. R. O. Morrison, D. Sotiriou, R. Stroynowski and $H$. Wahl (CERN).

This modified proposal requests 50,000 pictures of $\pi^{-}$into the 30" H.B.C. at $100 \mathrm{GeV} / \mathrm{C}$. The aims of the experiment are to study missing mass spectra, diffraction dissociation and correlations. The energy dependence of these effects will be studied, in particular by using lower energy $\pi p$ data already obtained by this group at 8,16 and $23 \mathrm{GeV} / \mathrm{c}$. The analysis would be greatly helped by the use of a Cerenkov counter and hodoscope system upstream from the chamber. 


\section{0-Inch $\pi^{-}-p$ e $200 \# 137$$$
-61-
$$ \\ STUDY OF $\pi^{-}+$p INTERACTIONS AT HIGH ENERGY}

Lawrence Berkeley Laboratory, University of California, Berkeley, Frmilab, Caltech F.R. Huson (spokesman)

This is a proposal for the bare 30 " to be exposed to $\pi^{-}$at $200 \mathrm{GeV} / \mathrm{c}$. About 10,000 interactions are requested $(50,000 \mathrm{pix})$. This is an exploratory experiment to search for new phenomena and study diffraction dissociation.

Summary reviewed by

F. R. Huson, February 1975 
STUDY OF MULTI PARTICLE PRODUCTION IN A 30 " BUBBLE CHAMBER

J. Chapman, J. Cooper, N. Green, B.P. Roe, A. Seidl,

D. Sinclair, J. VanderVelde (Univ. of Michigan); J. Biel,

J. Bradick, C. Bromberg, T. Ferble, P. Slattery,

B. Wormington (Univ. of Rochester); J. Sanders (BNL)

The Michigan and Rochester bubble chamber groups are proposing a collaborative investigation of $\mathrm{pp}$ interactions at high energy. This proposal is for a general survey using the bare hydrogen bubble chamber. The request is for 50,000 pictures with a proton beam at each of two energies - 100 $\mathrm{GeV} / \mathrm{C}$ and $400 \mathrm{GeV} / \mathrm{c}$.

The purpose is to extract the energy dependence of the total cross section and of multiparticle and diffractive production processes; to measure such energy dependence, the analysis must be carried out using uniform scanning rules and normalization techniques, consequently, Michigan and Rochester will in effect function as a single group. In addition to looking for unusual events by means of a very rapid scan by physicists, the intent is also to measure all topologies and test current theoretical models of hadronic interactions.

Summary reviewed by

J. VanderVelde, January 1976 
STUDY OF PP INTERACTIONS IN THE ANL 30-INCH HYDROGEN BUBBLE CHAMBER AT NAL

G. Charlton, Y. Cho, M. Derrick, R. Engelmann, T. Fields, L. G. Hyman, B. Musgrave, H. Yuta (ANL); L. Voyvodic, R. J. Walker (NAI); Z. Ming Ma (Michigan State Univ.); H. B. Crawley (Iowa State Univ.); B. Kehoe (Univ. of Maryland)

Surumary:

This is a proposal for the first 50,000 pictures of the 30-inch chamber to be taken at NAL. The proponents desire the highest energy proton beam available when the chamber is operational. They give two major purposes for their experiment. First, they are responsible for putting the chamber into working order at NAI and these pictures would help them check out the readiness of the reassembled system and insure that the precision of the chamber has been realized. Second, since they are so familiar with the characteristics of this chamber they will be able to rapidly explore the particle physics contained in this initial experiment. The whole effort of the ANL film analysis facility will be utilized for the short period of this experiment. They expect preliminary results within one month and final results within three months of the run.

(FRH)

Received: June 25, 1971

Summary Revised: May 30, 1972

8/26/71 Approved 50K pictures pp highest available energy at turn-on of $30^{\prime \prime}$ and beam. 


\section{PROPOSAL FOR A SEARCH FOR SUPERHEAVY ELEMENTS BY IRRADIATIONS AT NAL}

J. Halperin, O.L. Keller, R.J. Silva, R.W. Stoughton (ORNL); K.L. WOlf (ANL)

This group proposes to use an existing multiple neutron detector consisting of $20 \mathrm{He}^{3}$ counters to look for superheavy elements in samples previously irradiated in a high energy proton beam. They assume that elements in the hypothetical transuranic island of stability above the known periodic table will have a large neutron excess and a large spontaneous fission energy and hence should give rise to high multiplicity $(\sim 10)$ neutron events; several theoretical studies support this assumption. They will search for elements produced by such mechanisms as those suggested in the Marinov experiment. They are flexible in the sample goemetry and location provided the samples can be fit into the 9-inch 18
diameter by $30-i n c h$ long sample volume. Exposures of 10 or more protons are desirable. They have previously searched samples exposed at a level of $10^{17}$ lower-energy $(\sim 25 \mathrm{GeV})$ protons. The method is non-destructive and hence would allow target-sharing collaboration with others.

Physics objective: Search for superheavy elements.

Summary reviewed by R.W. Stoughton, January 1976 
PROPOSAL FOR A RAPID SYSTEMATIC STUDY OF ALL INTERACTIONS IN A $\pi$ EXPOSURE OF THE BARE $30 "$ CHAMBER AT $120 \mathrm{GEV} / \mathrm{C}$

P. L. Connolly, G. R. Kalbfleisch, R. C. Strand, V. VanderBurg (Brookhaven National Laboratory)

This is a proposal for 50,000 pictures of the bare 30 -inch hydrogen bubble chamber exposed to a $\pi$ beam. The experimenters expect to use a $300 \mathrm{GeV} / \mathrm{c} \pi^{-}$beam.

Their purpose in doing the experiment is both to test current theories of the strong interaction and to look for new effects at higher momentum.

They expect to have preliminary results within six months of receiving the film and final results in one year.

Summary reviewed

R. Strand, Feb. 1975 
Super Heavy Elements 147

PROPOSAL OF AN EXPERIMENT ON THE FISSION OF VERY HEAVY NUCLEI INDUCED BY 200 PROTONS IN PLASTIC DETECTORS

M. Debeauvais, J. Ralarosy, G. Remy, J. Tripier (Strasbourg)

This group wishes to study binary and ternary fission and fragmentation of heavy elements induced by $200 \mathrm{GeV}, 300 \mathrm{GeV}$ and $400 \mathrm{GeV}$ protons. They will use U, Th, Bi, Pb and Au targets evaporated on makrofol detectors and sandwiched between layers of makrofol plastic. After etching the emitted heavy nuclear fragments can be observed in $4 \pi$ geometry and measure angular distribution and ranges. It is proposed to irradiate 2 stacks of 10 samples of different heavy elements. This requires an integrate flux of $10^{10}$ to $10^{11}$ protons and a defocused beam uniform over a few $\mathrm{cm}^{2}$. The targets are 20 to 100 micrograms pro $\mathrm{cm}^{2}$ thick.

Physics objectives : Cross sections measurement of fission with two or more fragments in relation with the deposit energy of the incoming proton.

\section{First Results}

The experiment was performed on June $11^{\text {th }} 1975$ with protons of $300 \mathrm{GeV}$ in the meson area. The makrofol sandwiches are developped and the scanning is beginning. The first result gives a fission cross section for Uranium targets of $\sim 850 \mathrm{mb}$ and 2 ternary cross section of about $11,6 \mathrm{mb}$. The scanning of the other targets are in progress. 


\section{A STUDY OF NEUTRINO INTERACTIONS WITH NEUTRONS AND PROTONS USING THE 15-FOOT BUBBLE CHAMBER AT FERMILAB FILLED WITH DEUTERIUM}

C.Y. Chang, R.G. Glasser, D.G. Hill, B. Sechi-Zorn, G.A. Snow, G.B. Yodh (Univ. of Maryland); R. Burnstein, C. Fu, D. Petersen and H.A. Rubin (Ill. Inst. of Tech)

This experiment uses the 15' bubble chamber with a deuterium filling, a broad-band horn-focused neutrino beam from $200 \mathrm{GeV}$ proton interactions. (The proton energy could as well be $300 \mathrm{GeV}$ if that energy is more convenient from the scheduling point of view.) No auxiliary muon or photon detectors are provided, but the group is amenable to collaborate with other groups that can provide downstream detection equipment. The proposal stresses the physics that can be obtained from the analysis of a 300,000 picture initial exposure which should yield roughly 25,000 vn and $25,000 v p$ interactions. The neutrino flux can be determined directly from the elastic $\nu p \rightarrow \mu-p$ events seen in the bubble chamber.

$$
\text { The physics possibilities in this experiment are: }
$$

1. New particle search, especially for "charmed" particles.

2. Elastic form factors and flux calibration.

3. $\nu-n$ and $\nu-p$ inelastic cross section comparison for the dominant charged current interaction.

4. Adler Tests of PCAC.

5. Limits on the neutral current event rate for $v-n$ and $\nu-p$ interactions.

Summary reviewed by

G.A. Snow, January, 1975 
PROPOSAL TO BUILD AN ELECTRON-PHOTON FACILITY AT NAL AND TO MEASURE PHOTON SCATTERING AT HIGH ENERGIES

D.C. Cheng, D.E. Dorfan, S.M. Flatte, C.A. Heusch, H. Meyer, A. Seiden, A. Breakstone, A. Grillo, V. Palladino (University of Calffornia, Santa Cruz)

Sumary: It is proposed to assist NAL in the setting up and testing of an electron-tagged photon facility; to test and optimize high-energy electron and photon detection devices; and to perform basic measurements on photon scattering.

Beam: An electron beam has been designed by our group and others to provide for energyand direction-tagged photons of energies up to $250-300 \mathrm{GeV}$. Hadronic contamination is negligibly small. Available phoţon fluxes vary rapidly with energy; they are, under reasonable assumptions, above $10^{5}$ photons/pulse at the highest energies considered here. Deficiencies in primary photon flux or energy can be easily absorbed by means of a shift in useful photon energy.

Apparatus: The basic detector consists of shower spectrometers/hodoscopes spanning a large solid angle, plus a $\Delta \phi=2 \pi$ recoil detector at large angles in the laboratory. The shower detector is sectioned such that highest precision is gained, at highest rates, in the forward direction. It consists basically of sandwich-type modules with active converters, interleaved hodoscope devices, and summed energy information. It is a state-of-theart detector for all showering particles, and adequate for hadron detection. The recoil detector serves to catch mainly the slow nucleons recoiling from a diffractively scattered or produced forward particles. No magnets are used.

Objectives: Determination of mostly hadronic features of real photons (to implement virtual photon work in muon beams, etc.).

Basic Measurement: Compton effect, elastic and "inelastic" $(\gamma N+\gamma+$ hadrons). In 350 beam hours, from 10,000 to 20,000 elastic events and up to 1,000 inelastic events can be fully determined. Elastic slope parameters will be determined to a statistical accuracy of $<5 \%$ in reasonably spaced intervals (better than present determinations). The Inelastic Compton graph with its parton theory implication can be firmly established or disestablished with reasonable assumptions.

Meson Production: As a by-product, radiatively decaying mesons will be detected; there will be clearly distinguished signals for inclusive $\pi^{\circ}, \omega^{\circ}, \eta^{\circ}$ production; each of these has special significance for inclusive phenomenology.

Hunting Novelties: Many of the more exotic, and hitherto unseen, particles of the theorist dream zoo have distinctive radiative (e or $\gamma$ ) decays: the intermediate boson, heavy leptons of various types, heavy photons, magnetic monopoles. Pair production from a clean kinematic initial state may mean a unique path to their detection. In such a case, they will figure in our "backgrounds" free of charge. In particular radiative decays of the $\Psi$ particles may be observed. 
Brown Univ,, Univ, of Cinn., Univ, of Illinois, Ill. Inst. of Tech., Indiana Jniv., Hohns Hopkins Univ., INAL, ORNI, M.I.T., Rutgers Univ., Stevens Univ., Univ. of Tenn, anç Yale Univ.

\section{I.A. Pless (Spokesman)}

Phase I: It is proposed to build an electronic beamdefining anc tagging system to become a facility with the 30 " bubble chamber. The system will identify and tag incoming beam particles. In adcition, the direction and momentum of eaci tagged particle will be measured and recorded.

The system includes Cerenkov counters, proportional planes, and data collection equipment, including interfaces, readouts, magnetic tape units and small conputer. It is proposed to install, debug, operate, and document the system (botil haraware and sofiware) before turning it over for use as a facility.

Phase II: In this phase, cownstream proportional chamber and associated equipment are added, to permit the analysis of complex evants.

Phase III: In this phase, it is proposed to take a 500,000 picture exposure. These will be taken at the single momentum ci $200 \mathrm{GeV} / \mathrm{c}$ with 200,000 pictures taken with incident $\pi^{-}$mesons anci 300,000 pictures taken with a mixture of incident $\pi^{+}$mesors and protons. 
DEVELOPMENT OF THE PHASE I EXTERNAL MUON IDENTIFIER (EMI) FOR USE WITH THE NAL $30 \mathrm{~m}^{3}$ BUBBLE- CHAMBER

R. J. Cence, F. A. Harris, S. I. Parker, M. W. Peters, V. Z. Peterson, V. J. Stenger (Univ. of Hawaii); A. Barbaro-Galtierf, G. Lynch, J. P. Marriner, M. L. Stevenson (Lawrence Berkeley Laboratory)

The purpose of the muon identifier is to identify which of the final state particles in neutrino-induced reactions is the muon. Processes requiring muon identification include deep inelastic scattering, for both charged current and neutral current processes, dilepton (e $\mu$ and $\mu \mu$ ) events, and all "unusual" events.

The Phase I EMI proposal includes (1) a passive internal absorber $(\approx 3$ collision lengths) filling the space between the bubble chamber coils and matching them in effective thickness, and (2) an active external detector module that provides $x, y$ and diagonal coordinates at a single plane for each emerging track. This is located just outside the bubble chamber vacuum tank. This combination of absorber and detector has a measured rejection efficiency for $3 \mathrm{GeV}$ pions of $94 \pm 1 \%$, and an acceptance of $96 \%$ for muons. At higher momenta the angular acceptance improves but hadron "punch-through" increases.

The present Phase I EMI includes 25 modules of about $1 \mathrm{~m}^{2}$ each. Delay-line readout minimizes the cost of the electronics. The initial coll-absorber has been thickened with zinc to interpose 3.5 pion interaction lengths $\left(515 \mathrm{gm} / \mathrm{cm}^{2}\right)$ to the average hadron.

EMI performance is being evaluated using neutrino events from E-45 (hydrogen) and E-28 (neon-hydrogen) neutrino exposures. A paper is in preparation. An EMI "School" was held in October 1975 for 60 interested users. 


\section{STUDY OF SECONDARY PARTICLES PRODUCED BY 200 AND}

500 GEV PROTONS IN EMULSION CHAMBERS

K.NIU (UNIV, OF NAGOYA)

T.OGATA (I.N.S. UNIV. OF TOKYO)

\section{SURMARY OF PROPOSAL}

The purpose of these exposures are 1 . To examine the nuclear interactions produced, in order to validate current methods of inferring primary energies from observations on secondaries. 2. To study the $\gamma$ ray spectrum and the elasticity of collisions. 3. Search for short-lived particles previously observed by the author in cosmic rays. 4. Investigation of high energy nucleon nucleus interactions.

For these proposes emulsion chambers consisting of emulsion absorber assemblies will be used.

\section{STATUS}

COMPLETED: September, 1972

EXPER IMENTAL RESULTS AND PUBLICATIONS.

1) DETAILED ANALYSIS OF JET SHOWERS OBSERVED BY ERTLSION CHAMBERS WITH PRODUCING LAYER.

S. Kuramata et al.

CONEERENCE PAPERS 13TH INTERNATIONAL COSNIC RAY CONFERENCE 3, (1973), 2239

Calibration of the relative scattering method for determination of momentum of charged particles was done by means of emulsion chambers exposed to protons with momentum of $205 \mathrm{GeV} / \mathrm{c}$ at the Fermi National Accelerator Laboratory. The method was applied to the study of super high energy jet showers induced by cosmic rays.

2) X PARTICLE PRODUCTION IN 205 GEV/C PROTON INTERACTIONS.

K. Hoshino et al.

Prog. Theor. Phys. 53, (1975), 1859 and DPNU-3 Feb. 1975

Among secondary particles of $205 \mathrm{GeV} / \mathrm{c}$ proton interaction two possible candidates of short lived particle were observed. One is neutral which show clear 2 body decay and the other is charged one. Estimated life time and mass of those particles are not inconsistent with the $X$ particlestobserved in cosmic ray jet showers.

* K.Niu et a1., Prog. Theor. Phys. 46, (1971) 1644 


\section{PROPOSAL TO SURVEY HIGH ENERGY PROTON COLLISIONS IN NEON AND TO SEARCH FOR ANOMALOUS PHOTON BUNDLES AT NAL}

A. Benvenuti, U. Camerini, A.R. Erwin, W.F. Fry, M. Robinson, and M.A. Thompson (Univ. of Wisconsin)

Using the $30^{\prime \prime}$ bubble chamber filled with neon-H2 mixture they wish to (I) Search for anomalous interactions of high energy protons producing multiple photon events, (2) Obtain information on the $\pi^{\circ}$ production by high energy protons, (3) Search for nuclear collisions between protons and Ne that involve large target masses. They request 50,000 pictures of the Ne bubble chamber at the highest available proton momentum with $(2-5)$ protons per pulse. 
PROPOSAT FOR A STUUY OF TITE ITTSRACTION OF HIGII ENERGY $\pi \stackrel{ \pm}{ \pm}$ VITH NITON

W. D. Walker, L. Fortney, J. Loos, A. T. Goshaw, W?. Robertson (Duke University); G. W. Meisner ard R. B. Muir (University of IJortin Carolina)

They propose to stuày $\pi^{-}$interactions in the 30" hybria (chamber plus wice gap sparli chamber) system with hycirogerneon in the bubble chaniber. They want $50 \mathrm{~K}$ pictures with $4 \pi^{-} /$ picture and rot more than 20 per cent neon. ithe purpose of the experiment is:

1) To determine the multiplicity distribution in neon and compare this with data obtained in hyarogen.

2) To stuciy the multiplicity of $\pi^{O}$ 's produced in $\pi^{-}$-p interactions.

3) To look at the inclusive momentum distributions of proauced pions and compare hyarogen anci neon.

4) To search for Quarks.

j) To search for electromagnetic sizowers.

6) To study diffraction dissociation on neon.

7) To look at the propoerties of stoppirg tracls -- to sce whether exotic stable particles are produced.

Sumuary reviewed by W. D. Walker, April 1975 
PROPOSAL TO MEASURE TWO-BODY AND QUASI TWO-BODY ELASTIC SCATTERING AT LARGE T-VALUES AND IN THE U-CHANNEL

R.L. Anderson, D. Gustavson, K. Rich, D.M. Ritson, A. Weitsch (SLAC); R. Prepost (Wisconsin); B. Gottschalk (Northeastern)

Objectives: It is proposed to utilize the SASG focusing spectrometer facility (NAI Proposal No. 96) to study two types of interactions:

1. Elastic scattering $\pi^{ \pm}, \mathrm{K}^{ \pm}, \mathrm{p}, \overline{\mathrm{p}}$ at incident energies of 50 to $175 \mathrm{GeV}$ to $t$-values of $5(\mathrm{GeV} / \mathrm{c})^{2}$.

2. Quasi-elastic processes both close to the elastic limit and over the triple Regge region.

In addition we would expect to make preliminary runs on elastic and inelastic u-channel scattering preparatory to main physics running.

These runs would complement and supplement the Experiment 96 running and would hopefully give a complete description of overall and moderate $t$ scattering in the region close to the kinematic boundaries.

Experimental Technique: The focusing spectrometer will tag with Cerenkov counters incident beam and outgoing secondary particles. Its resolution of \pm 0.038 will play a strong role in kinematic separation of elastic and various quasi-elastic final states. For the u-channel reactions, where additional resolution is needed, it is planned to utilize a recoil side arm with crude magnetic analysis.

It is planned to spend the bulk of the time on items (I) and (2) and would expect to make rough preliminary u-channel measurements with a view to making a subsequent "carryon" proposal for good u-channel coverage.

The proposal requests 800 hours of beam. 


\section{PROPOSED EIUULSION EXPERIMENT SEARCH FOR} SHORT LIVED PARTICLES AT HIGH ENERGIES

P. Kotzer and J. J. Lord (Univ. of Washington)

\section{Summary:}

This emulsion proposal is divided into two parts. The first is concerned with a search for massive new particles with ljfetimes between $10^{-17}$ and $10^{-12} \mathrm{sec}$. and also includes an $8 \%$ measurement of the $\pi^{\circ}$ lifetime. An emulsion stack $7 \mathrm{~cm} \cdot \times 15 \mathrm{~cm} \cdot \times 3 \mathrm{~cm}$. is to je exposed to $90 \mathrm{~K}$ protons $/ \mathrm{cm}^{2}$ at the highest available NAL energy. It is planned to examine $5 \mathrm{~K}$ interactions for evidence of a decay or pair production within $1 \mathrm{~mm}$. of a point of interaction. Interest in this search has been stimulated recentiy by a Japanese cosmic ray emulsion experiment with a suggestion of a new particle.

A second portion of the proposal uses a five pellicle production stack followed by a multi-layered pb sheet - pellicle sandwich. The objective is to search for new particles decaying to $\gamma$-rays. A particle flux of $10 \mathrm{~K}$ protons $/ \mathrm{cm}^{2}$ is requested.

(AFG)

Received: April 19, 1972

Summary Prepared: June 8, 1972 
ANTINEUTRINO INTERACTIONS IN THE $15^{\prime} \mathrm{H}_{2}$-NEON BUBBLE CHAMBER

H.H. Bingham, W.B. Fretter, G. Yost (Univ. of Calif., Berkeley); H.J. Lubatti, K. Moriyasu (Univ. of Washington)

It is proposed to study $\bar{v}$ interactions in the 15-foot bubble chamber filled with $\sim 30 \%$ neon, $708 \mathrm{H}_{2}$. The experimenters are approved for $50 \mathrm{~K}$ pictures using broad band focusing. They propose to study:

1. Possibly the coherent interactions on neon.

2. Deep inelastic events and compare with the Exp. \#28A study of $v$-neon interactions to obtain $w_{3}$.

3. Dependence of $\sigma_{T}$ on energy and $A$. Compare with \#28A.

4. $\Delta S=\Delta Q$ in single strange particle production.

5. New particles and phenomena, including $\psi$, $\psi$ etc., sensitivity via $e^{ \pm}, \mu^{ \pm}, \gamma$ detection.

These groups have experience with heavy liquids including neon-hydrogen.

The experimenters hope to work with the LBL-Hawaii group in using information from the EMI to improve their $\mu^{ \pm}$ sensitivity, and with the Wisconsin group (Exp. \#28A) to compare $v$ and $v$ interactions in the same chamber, same liquid mixture, same EMI configuration. 


\section{EARLY MEASUREMENT OF HIGH ENERGY PP LARGE ANGLE ELASTIC SCATTERING}

J. Hartmann, J. Orear, J. Vreislander (Cornell); S. Conetti, D. Staris, C. Hojvat, D. Ryan, J. Trischuk (McGill); M. Gettner, W. Faissler, B. Gottschalk, J. Johnson, T. Kephard, D. Potter (Northeastern); P. Baranov, S. Rusakov (Lebedev Physical Inst. and Cornell)

Physics Motivation: The proton-proton elastic differential cross section would be measured at $300 \mathrm{GeV} / \mathrm{c}$ in two runs/ $4<-t<10 \mathrm{GeV}^{2}$ and $10<-t<20 \mathrm{GeV}^{2}$. Results from the ISR at 500 and $1500 \mathrm{GeV} / \mathrm{c}$ equivalent suggest the cross section becomes independent of the energy, whereas it is decreasing with energy in the $30 \mathrm{GeV} / \mathrm{c}$ region. This experiment hopes to determine at what energy the energy dependence disappears. Also, IRS results show a very narrow and deep dip at $-t \sim 1.5$ $\mathrm{GeV}^{2}$. Other dips are expected in the region $|t|>4 \mathrm{GeV}^{2}$ and would be measured in this experiment.

Experimental Technique: The experiment fits in the ProtonWest area without modification. Two large aperture spectrometers will measure both the forward and recoil protons in coincidence. Momentum in each arm will be determined using proportional wire chambers with appropriate bending magnets (the recoil arm has a large amount of kinematical focusing). The entire angular distribution from $4<-t<10 \mathrm{GeV}^{2}$ is taken at one time. Intensities $-10^{11}$ /pulse will give 100 events per bin per week for cross sections $\sim 10^{-3} \frac{1}{6} \mathrm{~cm}^{2} / \mathrm{GeV}^{2}$. Phase I is a test of the forward spectrometer to insure that singles rates will be under one megacycle instaneous rate. Phase I was completed in September 1975. Phase II will be the "low" $t$ run. 


\section{A STUDY OF THE AVERAGE NULTIPLICITY AND MULTIPLICITY DISTRIBUTION IN HADRON-NUCLEUS COLLISIONS AT HIGH ENERGIES}

W. Busza, C. Young, M.I.T.; C. Halliwell, E.P. Hincks, Carleton University; J. Elias, F.N.A.L.

Experiment \#El78, as originally proposed, has been successfully completed. The results on the average multiplicity of charged particles in $\pi$-nucleus collisions at 100 and $175 \mathrm{GeV}$ were presented at the Topical Meeting on High Energy Collisions Involvirg Nuclei in Trieste, september, 1974. They will also shortly appear in the Physical Review Letters.

As a follow-up to these measurements it is planned to study the following:

a) the rapidity distributions in hadron-nucleus collisions at 50, 200 , and $175 \mathrm{GeV}$;

b) compare in detail the multiplicities in hadron-nucleus collisions for $\pi, k$, and $p$ inciajent hadrons;

c) study correlations in the multiplicities observed in the target and projectile fragmentation region for hadron-nucleus collisions.

This data is needed to differentiate between various models of the space-time development of particle production.

For these measurements, the beam will be incident on a nuclear target which will be surrounded by a 49 element hodoscope array. As before, particles in the projectile fragmentation region will be detected in a single high resolution cerenkov counter through the intensity of Cerenkov radiation.

The experiment will be located in the M6W beam line. Threshold and differential Cerenkov counter will be used to identify the incident particle.

It is estimated that 100 hours of prime time at $210^{4}$ particles/ pulse plus 50 hours of parasitic time will be required to complete the measurements. 
$\mathrm{K}^{+} \mathrm{P}$ Multiparticle Production in the NAL 15' Bubble Chamber at the Highest Possible Energy

\author{
A. R. Erwin, E. H. Harvey, R. Loveless and M. A. Thompson \\ University of Wisconsin \\ Madison, Wisconsin 53706
}

It is proposed to study $\mathrm{K}^{+} \mathrm{P}$ interactions using the NAL $15^{\prime}$ hydrogen-filled bubble chamber. Slight modifications will be made to the existing hadron beam to obtain an enriched sample of $\mathrm{K}^{+}$, and it is planned to observe the fast $K^{\circ}$ decays in the large downstream volume afforded by the 15' chamber. The physics interest involves a study of quark-quark interactions, particularly with the interaction of a strange quark which is flagged by the production of a leading $\mathrm{K}^{\circ}$. Information will also be gathered on inclusive reactions and on particle momentum spectra and correlation functions. Production of charmed mesons should be at least an order of magnituce greater $\left(\sim \cos ^{2} \theta\left(\sin ^{2} \theta_{c}\right)\right.$ for beams which supply a strange quark in the initial state.

The beam is to be designed and utilized as follows: $300 \mathrm{GeV} / \mathrm{C}$ protons will be incident on an initial target thereby producing $\pi^{-}$ at $200 \mathrm{GeV} / \mathrm{c}$. The $\pi^{-}$will then be incident on a second target where $\mathrm{K}^{+}$'s are produced and collected at about $160 \mathrm{GeV} / \mathrm{c}$. Such a beam might easily be obtained by switching the $\pi^{-}$in the neutrino train onto the present hadron target or by striking a second target at the first focus of $N 5$ in enclosure 103. It is estimated that 1012 protons would yield $2 \mathrm{~K}^{+}$'s in the $15^{\circ}$ chamber with a $\mathrm{K}^{+} / \pi^{+}$ ratio of 1:I when the second method is used. This should be an ideal flux. Use of the 15' chamber assumes a Cerenkov counter and a minimum tagging system.

A request for $300 k$ pictures with 4 tracks per picture (one of which is $\mathrm{a} \mathrm{K}^{+}$) is expected to yield approximately $3 \mathrm{~K} \mathrm{~K}^{+}$interactions with a visible $\mathrm{K}^{\circ}$ decay. 


\section{A STUDY OF ANTINEUTRINO INTERACTIONS IN THE FERMI LAB 15-FT BUBBLE CHAMBER, FILLED WITH HYDROGEN AND NEON}

Fermilab, ITEP-Mosccrv, IHEP-Serpukhov, Univ. of Michigan, F.A. Nezrick (Spokesman)

In a collaboration of USSR and USA physicists it is proposed to study $\bar{v}$ interactions in the 15-foot bubble chamber filled with a neon-hydrogen mixture. The approval is for $50 \mathrm{~K}$ pictures at the highest available accelerator energy to determine the best neon-hydrogen_mixture. At $350 \mathrm{GeV}$ these would yield approximately $3.75 \mathrm{~K} \bar{v}$ interactions. An external muon identifier would be used if available. This experiment can examine the following:

1. Total $\bar{v} \mathbf{N}$ cross section and energy dependence.

2. Structure functions of deep inelastic interactions.

3. Study of neutral current interactions; conservation law tests.

4. The existence of heavy leptons (mass up to $2-3 \mathrm{GeV}$ ) and the intermediate boson (mass up to 9-10 GeV) can be seen directly and much higher limits on the intermediate boson mass can be inferred from deep inelastic investigations.

5. Iimits on multifermion and weak-electromagnetic interactions.

6. Questions concerning purely leptonic weak interactions. 
THE DIRECT PRODUCTION OF ELECTRON PAIRS IN NUCLEAR EMULSION BY 100 AND $200 \mathrm{GeV}$ PROTONS

A.S. Cary, T.K. Oskarsson (Harvey Mudd College)

An exposure of emulsions to high energy protons is requested at NAL. The purpose of this exposure is for a study of production of electron pairs by protons interacting with the coulomb field of nucleus. The data can be used for a check at high energies of a fourth order process in quantum electrodynamics. The cross section for pair creation is expected to be approximately $10^{-25} \mathrm{~cm}^{2}$ at NAL energies. An intensity of $10^{4-10^{6}}$ protons $/ \mathrm{cm}^{2}$ is requested.

Summary sent to A.S. Cary for review, May 1973 


\section{A PROPOSAL OF TIE PHOTOEMULSION EXPERIMENT AT THE NATIONAL ACCELERATOR LABORATORY (BATAVIA)}

N.A. Dobrotin, G.B. Zhdanov, M.I. Tretyakova (Alma-Ata Moscow - Leningrad - Tashkent - collaboration)

Summary: A request is made for an exposure of two or three emulsion stacks to a beam of high energy protons at NAL. The interest in this exposure is based on the following:

1) A study of high energy interactions and comparison of predictions from a fireball model.

2) Measuring the energy dependence of the cross section for production of one or more charged particles. Similar analysis has originated from exposures by this group at CERN and Serpukhov and there is a strong interest to compare the NAL data with the lower energy results.

\section{(AFG)}

Proposal Received: May 20, 1972

Summary Prepared: November 7, 1972

Approved: August 25, 1972 (completed) 
SEARCH FOR A NEW CLASS OF PENETRATING MASSIVE PARTICLES AT C-O AND A MEASUREMENT OF DIRECT LEPTON PRODUCTION

A. Benvenuti, D. Cline, J. Curry, W.T. Ford, R. Imlay, A.K. Mann,

J. Pilcher, D. Bintinger, D.D. Reeder, C. Rubbia, L. Sulak,

P. Wanderer (Chicago-Harvard-Pennsylvania-Wisconsin Collaboration)

\section{Physics Motivation:}

Results from recently analyzed data from serpukhov and the ISR suggest that either direct muon production occurs at high $p$ transverse $\left(p_{\perp}\right)$, or that heavy particles which are stable or which decay into leptons are copiously produced. The production. of new particles can be sensitively observed by the observation of leptons at large $P_{\perp}$. Furthermore, an attendant threshold enhancement might provide an important signature of heavy particle production that is relatively independent of the detailed nature of the heavy particle. This is directly accomplished by means of the magnetic field ramp at NAL which allows a continuous sweep in energy and therefore a precision search for threshold effects. In addition, this experiment, using the internal target, has the unique features that:

(i) it allows a search for short-lived particles because of the compact nature of the detector ( $\leq 10$ meters), and (ii) it has the ability to extrapolate easily to zero decay length to eliminate background, and (iii) it utilizes the well-defined profile of the internal beam, and (iv) it has the ability to go to the highest energies of which the accelerator is capable.

Experimental Technigue:

The experiment utilizes a plunging, rotating polyethylene foil target at the Internal Target Area. Secondary particles are required to pass through a hadron absorber and a Cerenkov counter is used to search for penetrating particles with masses above $1.3 \mathrm{GeV}$. Subsequent momentum analysis is performed with an air gap magnetic spectrometer with scintillation counters and proportional chambers placed between. Lead glass Cerenkov counters at the end of the spectrometer separates muons from hadrons. The entire apparatus is located below the Main Ring beam pipe. The results of the experiment were published in Physical Review Letters: PRL 34, 982 (1975) and PRL 35, 72 (1975). 


\section{A PROPOSAL TO STUDY SMALL-ANGLE PROTON-DEUTERON SCATTERING}

Y. Akimov, L. Golovanov, S. Muhkin, G. Takhtamyshev, V. Tsarev (Dubna); E. Malamud, R. Yamada, P. Zimmerman (NAL); R.S. Cool, D. Goulianos, H. Sticker (Rockefeller Univ.); D. Gross,

A. Melissinos, D. Nitz, S. Olsen (Univ. of Rochester)

\section{Physics Motivation}

Act I.

(1) Measurement of p-d elastic scattering slope parameter.

(2) Excitation of resonances at small momentum transfer. (Data taking completed.)

Act II.

Measurement of the deep diffractive dissociation of protons up to masses $M^{2}=100(\mathrm{GeV})^{2}$.

(Data taking in progress.)

Act III.

(1) Measurement of the real part of $\mathrm{p}$-d small angle scattering.

(2) Same for $\mathrm{p}-\mathrm{p}$ at $500 \mathrm{GeV}$.

(3) Attempt at large $t$ elastic cross section.

(Data taking after move of jet, August 1974)

Experimental Technique:

The apparatus for this experiment is nearly identical to that used for the earlier elastic p-p scattering work. The gas jet will be operated with deuterium. The recoil particle detector is extended to accept particles at $45^{\circ}$ in order to reach diffractive states of high mass. The deuterons will be positively identified by using thin $\mathrm{dE} / \mathrm{dx}$ detectors in conjunction with the thicker total energy detectors. In this fashion the background is greatly reduced and the deuteron signature verifies the coherent nature of the interaction. It is expected to measure $\mathrm{d}^{2} \sigma / \mathrm{dtdm}^{2}$ to an accuracy of $0.05 \mathrm{mb} /$ $(\mathrm{GeV})^{4}$ in the range of $0.02<t<0.10(\mathrm{GeV} / \mathrm{C})^{2}$ and $I<M^{2}<$ $100 \mathrm{Gev}^{\frac{1}{2}}$. The absolute value of the inelastic cross section is obtained by comparison with the elastic cross section. 
PHASE 0.8-SEARCH FOR LONG-LIVED MASSIVE OBJECTS

L.M. Lederman, J. AppeI, D. Saxon, I. Gaines, D. Hom, H. Paar, M. Borquin, D. Snyder (Columbia Univ.); T. Yamanouchi (Fermilab)

During some of the early work for NAI Experiment 70 , it has been proposed to utilize some beam time in a search for new high mass particles. These particles, produced by protons with energies from 200 to $400 \mathrm{GeV}$, could have masses between 5 and $25 \mathrm{GeV} / \mathrm{c}$ and Iifetimes $\gtrsim 10^{-7} \mathrm{sec}$. It is planned to have the primary proton beam strike a target in the tunnel adjacent to Enclosure E. Secondary particles would be produced and subsequently detected by a counter telescope approximately 1000 meters downstream in Proton Lab-Center. Relying on the r.f. bunching of the beam, it is expected to separate particles of various masses utilizing the differences in arrival time. Assuming $10^{9}$ interactions per pulse, it is expected to detect $10^{4}$ particles per pulse. It is planned simultaneously to use the beam for calibration of counters for later stages of their experimental program. 


\section{A PROPOSAL TO MEASURE CROSS SECTIONS FOR $\mathrm{pp} \rightarrow \mathrm{pX}, \mathrm{nX}$ AS A FUNCTION OF $S$ AND $M^{2}$ USING THE INTERNAL TARGET \\ FACILITY AT NAL}

J. Alspector, K. Cohen, G. Cvijanovich, T. Delillo, B. Maglich,

A. Pagnamenta, B. Robinson, F. Sannes and R. Stanek (Rutgers):

J. Carr, J. Keyne and̄ I. Siotis (Imperial College)

Physics Motivation: It is proposed to study the single particle inclusive reactions $\mathrm{pp} \rightarrow \mathrm{px}, \mathrm{pp} \rightarrow \mathrm{nX}$ and $\mathrm{pd} \rightarrow \mathrm{pX}$ and, in partjcular, to investigate the $s$ and $s / M^{2}$ dependence of the invariant cross sections. The data will be useful for a test of the triple Regge formalism and can be used to determine the relative magnitudes of: triple Regge couplings. This work can also be used for understanding various diffractive models.

Experimental Technique: The experimental apparatus for the most part includes an extension of equipment being used for an experjment at the Internal Target Laboratory. The target for the expeximent will be the currently available gas jet, running both with hydrogen and with deuterium. Recoil protons are measured in a counter telescope using range, pulse height, and time-of-fight. The counters used for absorbers of recoil protons will. also serve as detectors of recoil neutrons. A check will be possible of the data from the reaction $p p+n x$ and the time reversed reaction $\mathrm{pn} \rightarrow \mathrm{pX}$ after appropriate screening corrections are made to the deuterium data. Approximately 200 hours of running is requested to study reactions from hydrogen at energies up to $300 \mathrm{GeV}$.

\section{(AFG)}

Proposal Received: October 24, 1972

Summary Prepared: November 7, 1972

Status: Completed May 9, 1973 
NUCLEAR EMULSION EXPOSURES TO $400 \mathrm{GeV}$

David M. Ritson (Stanford Univ.)

It is proposed to expose nuclear emulsions to protons of energies 300-400 GeV. These plates would then be used by physics students in a laboratory course at stanford University. 
NAL PROPOSAL FOR STUDY OF PHOTONS AND LEPTONS PRODUCED IN MESONNUCLEON COLLISIONS IN THE DEEP SCATTERING REGION: SEARCH FOR INTERMEDIATE BOSONS, HEAVY LEPTONS, ANAMALOUS HADRONIC PROCESSES AND STUDY OF MULTI-GAMMA-RAY FINAL STATES

Z.G.T. Guiragossian, R. Hofstadter, R.F. Schilling, M.R.Yearian, (Stanford); E.V. Hungerford III, G.S. Mutchler, G.C. Phillips, (Rice); B. Cox, J. Peoples (NAL); B.W. Mayes (Houston)

The experimenters propose to construct a pion/electron beam facility at NAI and for this phase of their work to construct a spectrometer useful for the following:

1) Study of meson-nucleon collisions in the deep scattering region by observing large angle high energy produced gammarays, electrons and muons, singly or in pairs.

2) Search for new particles including intermediate vector bosons, Lee-Wick heavy photons and heavy leptons produced in deep meson-nucleon collisions. The effective mass of lepton pairs will be measured to better than 18 for particles with masses up to $22 \mathrm{GeV}$ produced by $300 \mathrm{GeV}$ pions. The particles would be observable in cases where the product of cross section and branching ratio is better than $10^{-37}-10-38 \mathrm{~cm} 2$.

3) Analysis of multi-gamma-ray final states from pion-nucleon collisions with resolution less than 28 .

The $100-300 \mathrm{GeV}$ pion beam originates from interactions of protons with energy of $400-500 \mathrm{GeV}$. The beam has the following properties: acceptance of $9.5 \mu$ ster, $\Delta p / p$ and momentum bite of \pm 28 and a spot size at the target of $\pm 2 \mathrm{~mm}$. Intensities of $10^{8}$ - $10^{9}$ pions/pulse are available from 1012 protons/pulse. Beam tagging will be done with one threshold and two differential Cerenkov counters. Analysis of events induced by kaons and antiprotons will also be possible at a rate of production of two orders of magnitude lower than by pions.

The spectrometer components are to be arranged in three configurations for each of the three phases of the experiment outlined above. The main and stationary component is an $18 \mathrm{kG}$ superconducting magnet with modules of multi-wire proportional chambers (MWPC). Other moveable parts include electron and photon identifiers using NaI (TI) absorption counters with MWPC's and muon identifier modules. The experiment is approved for 400 hours to study the production of high mass electron pairs and inclusive $\pi^{\circ}$ production from $\pi^{-}-p$ interactions. 
PROTON DEUTERON INTERACTIONS IN THE 30-INCH BUBBLE CHAMBER

S. Dado, A. Engler, G. Keyes, R. Kraemer, G. Yekutieli (Carnegie-Mellon); J. Lys, C.T. Murphy, M. Binkley (Fermilab); R. Diamond, A. Seidl, J. VanderVelde (Univ. of Michigan);

R. Engelmann, J. Hanlon, T. Kafka, M. Wahl (SUNY at Stonybrook)

Two exposures are approved for the 30-inch bubble chamber filled with deuterium and using the upstream and downstream proportional wire chamber systems. A total of 100,000 pictures are approved at each of two energies, $100 \mathrm{GeV}$ and $400 \mathrm{GeV}$. The analysis of this experiment will be compared with the results from runs at the same energies in hydrogen in which one of the collaborators was involved. The analysis will concentrate on the following topics:

1) Measurement of odd-prong multiplicity cross sections and comparison with even-prong results from the hydrogen data.

2) Determination of single-particle distributions. Studies can be made of isotopic spin exchange between the beam and target for reactions where pions are produced within a restricted kinematic region.

3) Study of two-particle correlations for a sample of events where the space angles of all secondary tracks are measured.

4) Study of neutron diffraction dissociation and double dissociation.

5) Measurement of the elastic and inelastic cross sections for particles coherently produced from deuterium.

6) Determination of neutral pion multiplicity and study of neutral strange particle production.

Summary revised by

C.T. Murphy, February 1975 
PROPOSAL TO MEASURE THE LIFETIME OF THE NEUTRAL PION

Y.K. Lim (Univ. of Singapore); Bernardi (Emmanuel College); K. Fukui (AFCRL); P.S. Young (Mississippi State)

An experiment is proposed to measure the lifetime of the neutral pion using nuclear emulsions. Using $\pi^{\circ}$ production by 200 or $300 \mathrm{GeV}$ protons interacting in the emulsion material, it is planned to study decays into Dalitz pairs. Pion decay lengths are expected to be 20-30 microns in an emulsion with a resolution of $\sim$ micron. Approximately $100 \mathrm{Dalitz}$ pairs are said to be necessary for a lifetime measurement with a 258 error. Three emulsion stacks are to be exposed with intensities of approximately $10^{5}$ protons per $\mathrm{cm}^{2}$.

Summary sent to Y.K. Lim

for review, May 1973 
PROTON DEUTEFON INTERACTIONS IN THE 30-INCH BUBBLE CHAMBER

S. Dado, A. Engler, G. Keyes, R. Kraemer, G. Yekutieli

(Carnegie-Mellon); J. Lys, C.T. Murphy, M. Binkley (Fermilab);

R. Diamond, A. Seidl, J. VanderVelde (Univ. of Michigan):

R. Engelmann, J. Hanlon, T. Kafka, M. Wahl (SUNY at Stonybrook)

Two exposures are approved for the 30-inch bubble chamber filled with deuterium and using the upstream and downstream proportional wire chamber systems. A total of 100,000 pictures are approved at each of two energies, $100 \mathrm{GeV}$ and $400 \mathrm{GeV}$. The analysis of this experiment will be compared with the results from runs at the same energies in hydrogen in which one of the collaborators was involved. The analysis will concentrate on the following topics:

1) Measurement of odd-prong multiplicity cross sections and comparison with even-prong results from the hydrogen data.

2) Determination of single-particle distributions. Studies can be made of isotopic spin exchange between the beam and target for reactions where pions are produced within a restricted kinematic region.

3) Study of two-particle correlations for a sample of events where the space angles of all secondary tracks are measured.

4) Study of neutron diffraction dissociation and double dissociation.

5) Measurement of the elastic and inelastic cross sections for particles coherently produced from deuterium.

6) Determination of neutral pion multiplicity and study of neutral strange particle production. 


\section{A PROPOSAL FOR A MAGNETIC RECOIL SPECTROMETER FOR THE GAS JET TARGET}

D. Nitz, S. Olsen and D. Gross (Rochester); K. Abe, R. Bomberowitz, K. Cohen,

P. Goldhagen, F. Sannes (Rutgers); D. A. Garbutt, R. Rusach, I. Siotis (Imperial College); and B. Morozov (Dubna).

\section{Motivation:}

The proposed experiment is a measurement of the $s$ dependence of $p-p$ elastic scattering in the region of the first diffraction minimum.

The primary questions addressed by this experiment are, for elastic p-p scattering:

1. Does shrinkage persist at high $t$ ?

2. How fast does the break seen at $t \simeq 1.2(\mathrm{GeV} / \mathrm{c})^{2}$ at $24 \mathrm{GeV}$ develop into a dip? Does it move as $s$ increases?

3. What is the energy dependence of the height of the secondary maximum observed at $|t| \sim 2.4$ ?

and for inelastic $\mathrm{p}-\mathrm{p}$

1. What is the $\mathbf{s}$ and $\mathbf{x}$ dependence of the inclusive proton spectrum for $|t| \geqslant 0.5 \mathrm{Gev} / \mathrm{c}^{2}$ ?

2. How does the production of the $N^{\star}(1520)$ and $N^{\star}(1688)$ as a function of $\mathrm{s}$ and $t$ compare with elastic scattering?

similar measurements could be made from deuterium.

\section{Technique:}

The recoil spectrometer will consist of a superconducting quad doublet and a superconducting dipole interspersed with 12 proportional wire planes. The function of the quads is to focus parallel rays from the target to a point at the first wire plane where the machine vacuum ends. Thus the recoil angle measurement is made using one point, is independent of target size and is unaffected by multiple scattering. The recoil momentum is measured by bending through $25^{\circ}$ in the dipole. Pions in the spectrometer will be rejected by time of flight. A Monte carlo estimate of the mass resolution at $200 \mathrm{GeV}$ and $t=1.5 \mathrm{GeV} / \mathrm{c}^{2}$ is $+137 \mathrm{MeV}$. The $|\mathrm{t}|$ range extends to $|t|=3.0 \mathrm{GeV}^{2}$ and the rate of elastic events at $|\mathrm{t}|=1.5 \mathrm{Gev}^{2}$ are $\approx 4 / \mathrm{pulse}\left(\mathrm{do} / \mathrm{dt}=10^{-32} \mathrm{~cm}^{2} / \mathrm{Gev}^{2}\right)$ with $10^{13}$ circulating protons. 
PROPOSAL TO SEARCH FOR WEAKLY PRODUCED MASSIVE IONG IIVED PARTICLES AT NAL

S. Frankel, W. Frati, L. Resvanis, and W. Yang, (University of Pennsylvania) and F. Nexrick (NAL)

\section{Summary:}

If new particles are produced at $>300 \mathrm{BeV}$ energies it is possible that they might possess new quantum numbers or that they are created by other than strong or electromagnetic interactions and hence are not copiously produced. Having new quantum numbers they might only decay with long half lives. one can search for such particles under "beam-off" conditions.

The key experimental requirement is to be met by a detector which can operate at extremely high radioactivity levels close to a large piece of target or beam dump and detect the decays of multi BeV particles above this high background and in the presence of cosmic rays.

\section{Procedure:}

In the first phase of this search a gas Cerenkov counter, possessing six phototubes, viewing independently the Cerenkov light from the same event, will be set at $\gamma>20$, well above the threshold for the most energetic electrons or $\gamma$ rays accompanying the radioactivity of the target.

Radioactivity background is suppressed by requiring a six fold coincidence to reduce accidental coincidences due to Cerenkov light in the quartz windows of the cerenkov counter.

The directional properties of the gas counter, aimed at the target from above, will be used to suppress cosmic ray background.

A thick accelerator target will be removed from the beam line and transported to our apparatus to search for activities in the hour-month range.

Summary reviewed by

5. Frankel, January 1976 


\section{SEARCH FOR TACHYON MONOPOLES IN COSMIC RAYS ABOVE 15-FOOT BUBBLE CHAMBER}

D.F. Bartlett, G. Schultz, D. Soo (University of Colorado); M.G. While, K.E. Wright (Princeton University)

Two of the most speculative hypothetical particles are the magnetic monopole and the tachyon. It is believed that these particles exist as a combined "tachyon monopole" and the experimenters propose using the fringing field above the bubble chamber as a detector. It is assumed that a tachyon monopole will emit Cerenkov radiation when it is accelerated by a longitudinal magnetic field.

This radiation is viewed with 8 photomultiplier tubes placed on the sides of a $14 \mathrm{ft}$. square by $8 \mathrm{ft}$. high box. The box is mounted directly below the bubble chamber roof. The bottom of the half of the box can "telescope" into the upper to permit passage of the crane.

Data taken during the winter of 1975 showed several candidate events. But two events also occurred when the magnetic field was off. Detailed calculations showed that extensive air showers (EAS) could occasionally produce sufficient scintillation light in the air filling of the box to simulate a tachyon monopole.

Accordingly, two plastic scintillator counters were installed in the box in May, 1975. The scintillators are large enough to record EAS's but small enough so that a legitamate tachyon monopole would not veto itself. In three weeks of running, we found that every time a large signal was registered by the phototubes viewing the box, the EAS counters also registered large pulses. Our inability to find any tachyonic events unaccompanied by an EAS enabled us to fulfill a goal of the experiment; namely, the lowering of the existing limit on tachyon monopoles in cosmic rays by a factor of 100 .

We did, however, find several double triggers: two events separated by a few microseconds. One event was accompanied by an EAs; the other apparently was not. Our apparatus was not set-up to record such double events. Modifications made during the summer of 1975 are permitting us to explore these double triggers.

Summary reviewed by

D. F. Bartlett, January 1976 


\section{EXPLORATION OF RARE MUON-INDUCED PROCESSES}

A.R. Clark, E.S. Groves, L.T, Kerth, S.C. Loken, M. Strovink, W.A. Wenzel (U.C. Berkeley - LBL); R. Cester, F.C. Shoemaker, P. Surko, M.S. Witherell (Princeton); R。P. Johnson (Fermilab)

Initial Physics Objectives will be pursued concurrently:

1) Search for heavy neutral muons predicted by gauge theories in the mass range $2-10 \mathrm{GeV}$, and other new particles decaying into one muon. The sensitivity for analyzed events is $1-2 \times 10^{9}$ events/ub.

2) Measurement of deep-inelastic virtual Compton scattering and search for other deep-inelastic $\mu^{+} \mu^{-}$pair creation.

3) Measurement of the deep-inelastic muon scattering structure function at very high momentum transfers. For example, 8200 events exceeding $Q^{2}=160(\mathrm{GeV} / \mathrm{c})^{2}$ will be obtained.

4) Collection of $2-3 \times 10^{4} \psi+\mu^{+} \mu^{-}$decays and data at higher dimuon masses. The $\psi \mu \mu$ coupling will be measured in the range $-15(\mathrm{reV} / \mathrm{c})^{2}<\mathrm{q}^{2}<0$ with $7 \%$ statistics at $\mathrm{q}^{2}=-\mathrm{m}_{\psi}{ }^{2}$ 。

\section{Experimental Technique}

The spectrometer will realize a broader range of muon physics objectives than those accessible to the first-round muon scattering experiments. It consists of 18 modules, each containing 5 magnetized iron plates, 5 calorimeter counters, 1 proportional chamber, and $1 \mathrm{drift}$ chamber. Alternate modules contain 1 trigger hodoscope. The necessary sensitivity to rare processes is provided by high acceptance over the full length of the distributed target-spectrometer. Nearly continuous iron is used to suppress $\pi \rightarrow \mu \nu$ decay background and provide the medium for calorimetric determination of energy transfer to hadrons. Fast detectors will tolerate further increases in beam intensity while maintaining acceptance of multiple finalstate muons even within the beam area. The various background trigger rates have been estimated and found to be manageable.

Requirements for Operation

Existing space in the north portion of the Muon Laboratory is required, with an illumination of $10^{12}$ muons at the highest available energy (225 GeV has been assumed). The experiment is compatible with normal operation of the Muon Scattering Facility upstream. 
PHENOMENOLOGICAI STUDY OF MUON-NUCLEON COLLISIONS AT $150 \mathrm{GeV} / \mathrm{C}$

IN EMULSION

O. Kusumoto, S.Ozaki and M.Teranaka (Osaka City University);

H.Okabe and J.Yokota (Science Education Institute of Osaka Prefecture);

M.Ohta (Kinki University); K.Shibata (Okayama University); S.Mikamo

(National Laboratory for High Energy Physics).

Summary:

The phenomenological features of charged hadrons produced by the muon-nucleon interaction at $150 \mathrm{GeV} / \mathrm{c}$ are examined and the results are compared with those obtained by the proton-nucleon interaction at $200 \mathrm{GeV} / \mathrm{C}$ and, then, any proper feature of particle production by a virtual photon is probed. The phenomenological features of charged hadrons, that is, multiplicity, emission angle, and so on are examined over the ranges of low- $\mathrm{q}^{2}$ to the scaling region and of $\mathbf{s}$ value up to $100 \mathrm{GeV}^{2}$ or more $\left(\mathrm{q}^{2}\right.$ is the squared 4-momentum transfer of the muon and $s$ is the squared mass of the produced hadron system). The $A$ dependence ( $A$ is the mass number of the target nucleus) of multiplicity and emission angles of charged particles are also examined and compared with the results for the proton-nucleus collisions.

Procedure:

a) Stack and Exposure Condition: Ordinary and sandwich stacks are used. An ordinary stack consists of G5 pellicles of 3 " $x 6^{\prime \prime} \times 600$ $\mu \mathrm{m}, 48$ sheets, and is exposed with a beam density, $5 \times 10^{5} / \mathrm{cm}^{2}$. A sandwich stack consists of metal foil and emulsion, each of which is alternatively piled up and is exposed with a density, $5 \times 10^{5} / \mathrm{cm}^{2}$. $A 1, F e$ and $W$ of thickness 200 to $250 \mu \mathrm{m}$ are used as sandwiched foils.

b) Scan and Measurement: Area scanning method is employed. The charged particle with the highest momentum is assumed as the outgoing muon. $\mathrm{q}^{2}$ and $\mathrm{s}$ are approximately evaluated by the momentum measurement of the outgoing muon or by summing up the energies of the produced hadrons. 
DEVELOPMENT OF A TRANSITION RADIATION DETECTOR FOR DISCRIMINATING BETWEEN PIONS AND KAONS AT NAL ENERGIES

F. A. Harris, T. Katsura, S. I. Parker, V. Z. Peterson, V. J. Stenger (Hawaii); R. E1lsworth, G. B. Yodh (Maryland)

The proposal is to develop a practical Transition Radiation (TR) detector which should complement but may exceed gas-Cerenkov $\pi / \mathrm{K}$ discrimination at the highest NAL energies. The Hawafi-Marylandoxford collaboration has already obtained good (signal/noise) ratios from electron-induced TR $x$-rays, using argon-, krypton-, and xenonfilled MWPC. Both "sandwich" array (Interleaved radiators and detectors) and "magnetic separation" array (deflected charged particles) have been tested experimentally at LBL and SLAC.

The proposed NAL setup (in the 30 " BC beamline) includes a single radiator (thin $B e$ and Li foils), magnetic separation with existing beamline magnets, and a multi-cell xenon-filled MWPC detector $\sim 200$ feet downstream. At $400 \mathrm{GeV} / \mathrm{c}$ pions are expected to produce a $\sim 100 \mathrm{KeV}$ signal ( $\sim 5.2$ photons detected); the kaon signal should be $\sim 6 \%$ of the pion signal.

D1rect comparison of TR with the gas Cerenkov is proposed.

The reduced multiple-scattering of pions (as compared with electrons) offer the interesting possibllity of observing for the first time "diffraction-grating" coherence in TR.

At present the Lithium-foil radiator has been assembled and a solidstate detector is under construction. The xenon-gas detector design has evolved into a drift chamber providing both spatial and pulse-height information. 


\section{PROPOSAL TO STUDY $500 \mathrm{GeV}$ PP COLLISIONS}

IN THE 30 INCH BUBBLE CHAMBER AT NAL

R. Engelmann, T. Kafka, J. Lee-Franzini, C. Moore and M. Pratap (SUNY at Stony Brook)

A 50,000 picture exposure of the 30 inch bubble chamber, filled with hydrogen, to a $500 \mathrm{GeV}$ proton beam is requested. About 5 tracks per picture will yield about 10,000 events. A thin lucite plate is to be inserted in the downstream part of the chamber to assist in track matching.

The measurements of interest are:

1) A $1.7 \%$ measurement of $\sigma_{\text {tot }}$ at $500 \mathrm{GeV}$ (from the ISR data one predicts a 58 rise).

2) Multiplicity distribution, its moments and their energy dependence.

3) Single particle inclusive spectra for negative particles $(\pi)$ in the complete backward hemisphere (y) and for all charges in both hemispheres (logtan $\left.\frac{{ }^{0} \mathrm{LAB}}{2}\right)$.

4) Neutral strange particle production and $\pi^{\circ}$ production ( $n_{\pi}$ o vs.n-charged).

5) Two particle correlations.

6) The diffractive process $\mathrm{pp} \rightarrow \mathrm{p}$ slow $+\mathrm{MM}^{2}$ and its energy dependence.

7) $\Delta^{++}$production and its energy dependence.

Summary prepared by R. Engelmann, May 1973 
A PROPOSAL TO STUDY PROTON-PROTON INTERACTIONS

AT THE ENERGY OF 300 GEV OR HIGHER

AT THE NAL BUBBLE CHAMBER

M. A. Markov, J. S. Takibaev

(Academy of Sciences of the Kazakh, U.S.S.R.)

A request has been made for 75,000 pictures of an NAL bubble chamber exposed to protons with energy of $300 \mathrm{GeV}$ or higher. The purpose of this experiment is to study high energy proton-proton interactions. It is proposed by a group from the U.S.S.R. which ltas experience studying hadron-hadron interactions both in emulsions and in bubble chambers.

Summary reviewed by

M. A. Markov, January 1976 


$$
\text { -100- 30-Inch PD @ } 300 \# 209
$$

\section{A STUDY OF $300 \mathrm{GeV} / \mathrm{C}$ Pd INTERACTIONS IN THE}

THIRTY-INCH BUBBLE CHAMBER

C. Peck, A.P. Sheng (Caltech); W.E. Anderson, H.B. Crawler, A. Firestone, W.J. Kernan, D. Parker (Iowa); J.M. Canter, F.T. Dao, W.A. Mann, J. Schneps (Tufts); R. Cassel1, S. Jones, R.S. Panvini, J.S. Poucher, A. Rogers, S. Stone (Vanderbilt)

Picture-taking for experiment E-209 was completed in October of 1975. The number of participating institutions and physicists has increased from that mentioned in the original proposal. The physics objective has also been altered slightly and expanded to include a careful study of the double diffraction dissociation process involving the beam proton and the target neutron. So far we have worked on the following topics:

1. Charged multiplicity distributions and the effect of double scattering in deuterium. Paper completed and published in A. Sheng et al., Phys. Rev. D12, 1219 (1975).

2. Inclusive distributions in the target fragmentation region using reactions $p+n \rightarrow h+x$, where $h$ includes the hadrons p, $\pi^{+}$, and $\pi^{-}$. Paper presented at the Washington APS meeting April 28 - May 1 (1975). Since that meeting we have doubled our reaction samples and are presently preparing a paper for publication. The paper will also probably include the next topic :

3. Excitation states from the neutron target fragmentation. Paper also presented at the Washington APS meeting, April 28 - May 1 (1975).

4. Analysis of the spin structure of diffractively excited states and the correlations with the charged multiplicity. Paper to be presented at the New York APS meeting, February 2-5 (1976).

5. Study of double diffraction dissociation process from threeto six-pronged events in pd interactions. We are scanning for, and measuring the double diffraction candidates in the 30" bubble chamber; the joining of these bare chamber measurements with tracks detected in the downstream hybrid spark chambers is presently underway.

We have also considered other possible physics projects such as a detailed study of double scattering within the deutron using two-proton events and a search for new heavy resonances which decay into $\mathrm{K}^{\circ}$ or o plus other charged particles. Detailed consideration will be given to these new projects as analysis of our initial five topics nears completion. 


\section{PROPOSAL FOR RADIATION MEASUREMENTS AROUND A PROTON BEAM DUMP AT $300 \mathrm{GeV}$}

H.O. Wuster, M. Growley-Milling, ㅌ. Goebel (CERN); M. Awschalom (NAL)

A request has been made for use of the primary proton beam to check some Monte Carlo calculations for the $300 \mathrm{GeV}$ CERN project to determine particle flux densities, radiation heising, and absorbed dose. All of these measurements are included in the approved NAI Experiment \#108 but it is doubtful that the results from that experiment would be available before these measurements are needed. The spokesman from Experiment \#108, M. Awschalom, will participate in this work.

The specific measurements can be broken down as follows:

1. Measurement of energy deposition.

Measurement of the temperature rise of a cylindrical beam stop; $10^{11}$ ppp for 4 hours.

2. Measurement of extranuclear cascade in a beam stop. Exposure of a number of steel plates with foils for activation analysis perpendicular to a proton beam with 1011 - 1012 ppp; 24-40 hours of exposure.

3. Measurement of beam losses and extranuclear cascade in Exposure of a main ring magnet to a proton beam of $10^{12}$ PpP for -10 hours.

Summary prepared by

A. F. Greene, June 1973 
PROPOSAL TO STUDY HIGH ENERGY

NEGATIVE HOPEROÑ INIERACTIOISS WITH

THE NAL 15-FOOT BUBBLE CHAMBER

Cambridge, U. of Michigan (Ann Arbor), Strasbourg

\section{J.G. Rushbrooke (Cambridge)- Spokesperson}

It is proposed to transport $10^{5}$ primary protons/pulse to the vicinity of the 15-foot bubble chamber in order to produce a negative hyperon beam suitable for analysis in the chamber. The hyperon beam would rely on the use of high field pulsed quadrupole magnets which have been supplied by the Rutherford Laboratory. The proton beam is incident on a heavy target. Pulsed magnets focus and bend the negative particles of interest through a series of collimators which comprise a total length of $6.5-13.0 \mathrm{~m}$ depending on momentum. The momentum resolution of the hyperon beam is $u \pm 5 \% \delta \mathrm{p} / \mathrm{p}$. $\Sigma^{-} / \pi^{-}$ratios vary from $6 / 4$ to $2 / 8$ at the chosen mowenta. Further selection of events can be made by requiring the observation of a secondary $\Sigma^{-}$decay.

The physics motivation is based on systematic studies of hyperon interactions including topological cross sections, multiplicity distributions, inclusive distributions and unanticipated phenomena. The specific request is for $50 \mathrm{~K}$ pictures of $200 \mathrm{GeV} / \mathrm{c}$ hyperons, $75 \mathrm{~K}$ pictures at $100 \mathrm{GeV} / \mathrm{c}$, and $150 \mathrm{~K}$ pictures at $50 \mathrm{GeV} / \mathrm{c}$. Within the 100 and $200 \mathrm{GeV} / \mathrm{c}$ exposures there are expected $\sim 1000 \Xi^{-}$and $\sim 50 \Omega^{-}$decays. 


\title{
STUDY OF $400 \mathrm{GeV} \pi^{-} p$ INTERACTIONS IN THE
BARE $30^{\circ}$ BUBBLE CHAMBER
}

\begin{abstract}
G. Abrams, C. Friedberg, G. Goldhaber, A.D. Johnson, J. Kadyk, G. Trilling, F. Winkelmann (LBL); H. Bingham, D. Chew, W. Fretter, W. Graves, L. Stutte, G. Yost (Univ. of Calif., Berkeley); D. Bogert, R. Hanft, F.R. Huson, D. Ljung, C. Pascaud, S. Pruss, W.M. Smart (Fermilab)
\end{abstract}

It is proposed to obtain a $50 \mathrm{~K}$ picture exposure of negative pions incident on the 30-inch hydrogen-filled bubble chamber. This work is proposed by the same group which obtained data of $\pi-p$ interactions at $200 \mathrm{GeV}$ in the 30 -inch chamber (Experiment \#137). With a primary beam momentum of $400 \mathrm{GeV}$ it is believed that the pion flux at $380 \mathrm{GeV}$ will be sufficient for the exposure. It is expected that about 800 elastic and 6,000 inelastic events will be available for analysis. The primary interests include measurements of total and elastic cross sections, multiplicity distributions, diffractive processes, strange particle production, inclusive distributions, double-Pomeron exchange processes, and characteristics of copiously produced channels. 


\section{A MEASUREMENT OF THE PION FORM FACTOR BY DIRECT PION-ELECTRON SCATTERING}

D. Drickey, C. May, E. Dally, H.K. Ticho, C. Buchanan, D. Stork, (UCLA) ; P. Shepard, (Univ. of Pittsburgh); J. Poirier, C. Rey, R. Wojslaw (Univ. of Notre Dame); A. Wehmann, T. Toohig, J. Tompkins, A. Lennox, (Fermilab).

The objective of this experiment is to measure the pion electromagnetic form factor in the spacelike region of momentum transfer by direct observation of pions from a beam scattered from stationary electrons in a hydrogen target.

A previous experiment conducted at Serpukhov yielded a pion radius squared of $(0.61 \pm 0.15) \mathrm{F}^{2}$. The initial data for the present experiment will be taken at $100 \mathrm{GeV}$, twice the Serpukhov value. The momentum transfer range will be extended to $0.08(\mathrm{GeV} / \mathrm{c})^{2}$ and thus the effect of pion size will be significantly increased and systematic errors reduced.

The apparatus is being installed in the Ml bean line at the Meson lab. The trajectory of the incident pion entering a $50 \mathrm{~cm}$ long liquid hydrogen target and the momenta and scattering angles of the recoiling $\pi$-e pairs will be measured with a combination of proportional wire chambers, spark chambers and bending magnets (2 BM 109's with $8^{\prime \prime} \times 18^{\prime \prime}$ aperature and $\left.\int B d 1=80 \mathrm{~kg}-\mathrm{m}\right)$. Electrons and hence the $q^{2} 1 \mathrm{abel}$ for each event will be identified through kinematic analysis of the recoiling particles made possible by the high angular precision of the apparatus $( \pm 0.2 \mathrm{Mr})$. A redundancy check on the electron identification is supplied by total absorption $\mathrm{Pb}$-glass shower counters and any $\mu-\mathrm{e}$ events are rejected in a muon filter.

An approved 100 hours for testing and running is consistent with the above objectives. We are further encouraged to subsequently rm at the highest beam energy practical in the Meson Area. We anticipate that $200 \mathrm{GeV}$ running will be practical and hope to thereby extend the pion form factor measurements to $\mathrm{q}^{2}=0.18(\mathrm{GeV} / \mathrm{c})^{2}$.

A measurement of the $K$ meson form factor is experimentally difficult at $100 \mathrm{GeV}$. We will attempt to make such a measurement to a precision of $20 \%$. It becomes considerably easier at $200 \mathrm{GeV}$ and we hope that the higher energy running proves practical.

D. Stork

January, 1975 
A COMPARISON OF 100 AND $200 \mathrm{GeV}^{+}-$p INTERACTIONS

J. Erwin, W. Ko, R. Lander, D. Pellett, P. Yager (Univ. of Calif., Davis); M. Alston-Garnjost (LBL); J.H. Friedman (SLAC)

An exposure of $50 \mathrm{~K}$ pictures of tagged $200 \mathrm{GeV}$ positive pions is proposed for the 30-inch hydrogen-filled bubble chamber. This would match a similar exposure at $100 \mathrm{GeV}$ (Experiment \#121A).

Summary prepared by

A. F. Greene, June 1973 


\section{PION-DEUTERON INTERACTIONS AT $200 \mathrm{GeV} / \mathrm{C}$}

J. Erwin, W. Ko, R. Lander, D. Pellett, P. Yager (Univ. of Calif., Davis); I. Dunn, H.J. Lubatti, K. Moriyasu, W. Podolsky (Univ. of Washington)

A proposal is made for $50 \mathrm{~K}$ pictures of the 30-inch deuteriumfilled bubble chamber exposed to a $200 \mathrm{GeV} / \mathrm{c}$ negative pion beam. Use would be made of a downstream spectrometer system if it were available. The purpose of the experiment is to utilize the properties of the deuteron to study reactions which cannot easily be studied on protons. This may include (1) inclusive reactions such as $\pi^{-}+n \rightarrow \pi^{ \pm}+$anything, (2) diffractive dissociation of the neutron, (3) diffractive dissociation of pions on deuterons.

Summary prepared by

A.F. Greene, June 1973 
S. Childress, P. Franzini (Columbia Univ.); J. Lee-Franzini, R. McCarthy, R. Schamberger (SUNY, Stony Brook)

It is proposed to move the apparatus used for NAL Experiment \#14A to the Internal Target Area. They have taken data at a location in the Neutrino Target Hall at energies of 200,300 , and $400 \mathrm{GeV}$. Their $200 \mathrm{GeV}$ results have been prepared for publication and indicate that the proton-proton inelastic cross section exhibits unusual behavior in the region of low $|t|$. At low $\mathrm{M}^{2}$ the cross section is small at small $|t|$ and appears to approach a maximum at $|t| \approx 0.1 \mathrm{Gev}^{2}$. They propose to extend their measurements to include a careful study of the s-dependence of the inelastic cross section. The target will consist of rotating polyethylene foils and carbon filaments. The basic detectors are solid state and enable

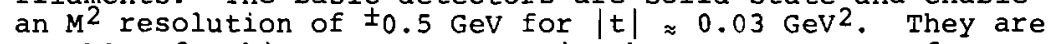
capable of making measurements in the momentum transfer region $0.02<|t|<0.2 \mathrm{Gev}^{2}$. 
STUDY OF DIFFRACTION DISSOCIATION (COHERENT AND INCOHERENT) AND INCLUSIVE PARAMETERS OF THE INTERACTION OF HIGH ENERGY PIONS AND PROTONS ON NUCLEI HEAVIER THAN HYDROGEN

L. W. Jones, J. VanderVelde and D. E. Lyon, Jr. (University of Michigan)

This experiment is proposed to study the multiplicities, momenta, and angular distributions of charged hadrons produced by protons and pions of $200 \mathrm{GeV}$ on nuclei. The objective is to make a systematic study of the inclusive parameters of strong interactions as functions of nuclear size (e.g. atomic weight). Such studies provide in principle a unique means of exploring the early evolution of the final state of strong interactions at very high energies, wherein the nucleus itself is used as a high-density micro-laboratory. A secondary motivation is to determine the phenomenological parameters of nuclear interactions in order to better interpret neutrino and cosmic ray data wherein direct knowledge of the interaction energy is unknown.

The experiment would employ a target assembly containing four small plates of $\mathrm{C}, \mathrm{Al}, \mathrm{Cu}$, and $\mathrm{Pb}$ within the sensitive volume of the 30-inch bubble chamber. Two sets of 50,000 pictures each are requested in order to have a sample of 4,000 interactions in each element with each beam. Interest in the results is enhanced if additional runs at divergent energies could also be obtained.

Summary reviewed by

I. W. Jones, January 1975 


\section{COHERENT $K_{S}^{0}$ REGENERATION BY ELECTRONS}

J. Roehrig, E.I. Rosenberg, V.L. Telegdi, B. Winstein (Univ. of Chicago); H. Kobrak, R.A. Swanson (Univ. of California, San Diego); S.H. Aronson (Univ. of Wisconsin); H. Hofer (Swiss Fed. Inst. of Technology, Zurich)

It is proposed to extend the use of the E-82 spectrometer in the neutral beam M4 to a determination of the amplitude for regeneration of $\mathrm{K}_{\mathrm{S}}$ by electrons. This quantity is directly proportional to the rms neutral kaon charge radius, $R^{2}$. NAL is uniquely suited for such a determination since the electron contribution to regeneration is $\varepsilon \approx 78$ at $100 \mathrm{GeV}$, and grows as the square root of the kaon momentum. It is proposed to measure this number to a precision $\Delta E \simeq 1.8 \%$. For this purpose they need to do some ancillary measurements which are also of interest:

a. Measurement of $\sigma_{\text {tot }}\left(K_{I}^{0}-n u c l e i\right)$ in good geometry to a precision $\leq 18$, by inserting material in the $\mathrm{K}_{\mathrm{L}}$-beam far upstream.

b. Measurement of the $\mathrm{k}^{0}-\mathrm{p}$ elastic scattering cross section, by measuring the angular dependence of $\mathrm{k} g$ events, and determining the $t=0$ point in absolute value from $\sigma_{\text {tot }}$ via the optical theorem.

c. Measurement of $\mathrm{K}_{\mathrm{S}}^{0}$ diffractively regenerated from a moderately thin regenerator.

d. Measurement of inelastic regeneration. One of the many ways possible is to trigger only on inelastic events.

They require $10^{5}$ regenerated events at $100 \mathrm{GeV} / \mathrm{c}$, which in principle could be taken in 5 days of good running. They estimate a need for 30 days of running time. 
PROPOSAI TO INVESTIGATE NEUTRINO INTERACTIONS

IN DEUTERIUM USING THE FERMILAB 15-FT BUBBLE CHAMBER

R. Engelmann, T. Kafka, J. Lee-Franzini, M. Pratap (SUNY at Stony Brook); J. Canter, W.A. Mann, J. Schneps, G. Wolsky (Tufts University)

A 300,000 picture exposure of the 15-foot bubble chamber to a broad band neutrino beam from 350 GeV protons is requested. The neutrino flux will be determined from elastic and $\Delta^{++}$production events to about $13 \%$. The $\mu$ can be identified in most of the cases without external $\mu$ identification and the final state hadron energy can be estimated on a statistical basis. The experimenters are interested though to collaborate with other groups for the use of an external $\mu$ identifier.

The main physics interest is:

1. Extension of elastic form factor fits to higher $q^{2}$ at Fermilab energies.

2. Simultaneous investigation of un and $v p$ inelastic reactions in one exposure with one neutrino flux, i.e., comparison between $\sigma(v n)$ and $\sigma(v p)$ and the $\mathrm{n}$ and $\mathrm{p}$ weak structure functions.

3. Search_for isotensor currents by measuring the ratio $\nu \mathrm{p} \rightarrow \mu^{-} \Delta^{++} / \nu_{n} \rightarrow \mu^{+}$in one experiment.

4. Search for and study $\Delta S=-\Delta Q$ transitions, neutral currents, new particles.

5. Tests of sum rules.

Realistic estimates of available neutrino fluxes suggest that a large number of pictures will be needed in order to answer the physics questions of interest. The experimenters therefore feel justified to propose an exposure similar to one already proposed. 
PROPOSAL TO EXTEND THE ENERGY RANGE OF A STUDY OF MULTIPARTICLE PRODUCTION IN PP COLLISIONS

J. Chapman, J. Cooper, N. Green, B. Roe, A. Seidl,

J. Vandervelde (University of Michigan); C. Bromberg, D. Cohen, T. Ferbel, P. Slattery, D. Underwood (University of Rochester)

The Michigan-Rochester Collaboration, in a pair of coupled experiments, has obtained a total of about 31,000 exposures of the bare 30-inch ANL/NAL bubble chamber to $100 \mathrm{GeV} / \mathrm{c}$ protons (Proposal 252) and 12,000 exposures of this chamber to $400 \mathrm{GeV} / \mathrm{c}$ protons (Proposal 138). Since their motivation has been largely to study the energy dependence of various production processes they wish to substitute the remaining pictures at $100 \mathrm{GeV} / \mathrm{c}$ for an equivalent exposure at $60 \mathrm{GeV} / \mathrm{c}$. This will provide valuable measurements of the total cross section and of multiparticle production processes to compare with Serpukhov and ISR experiments.

They will emphasize the analysis of the diffractive part of the inelastic cross-section.

Summary reviewed by

T. Ferbel, February 1975 
A PROPOSAL FOR TESTING A TRANSITION RADIATION DETECTOR AT NAL

G.F. Dell, Jr., R. Keller, H. Uto, Luke C.L. Yuan (BNL)

It is proposed to use a high energy beam containing hadrons and muons to study transition radiation detectors at NAL energies. Some measurements have already been made using electrons at $\gamma^{\prime}$ 's which simulate NAL energies but uncertainties remain about the bremsstrahlung background and coulomb scattering of heavier particles. The specific objectives of the experiment are as follows:

1. Measurement of the yield of transition radiation from muons and hadrons using a large number of proportional wire chambers (PWC's) filled with Xe at the highest energy available $(280 \mathrm{GeV})$ and comparison with theoretical estimates.

2. Study of the relativistic rise effect also using PWC's.

3. Measurement of the yield of transition radiation from a NaI crystal with a central beam hole.

The specific request from NAL is for a space of 10-15 feet along a beam line in the Meson Area.

Summary reviewed by

Luke C.L. Yuan, January 1975 


\section{A SEARCH FOR "SCHEIN EVENTS" AND \\ EVENTS WITH A HIGH MULTIPLICITY OF $\gamma^{\prime} s$}

D.L. Burke, H.R. Gustafson, L.W. Jones, and M.J. Longo

(University of Michigan)

Cosmic ray emulsion experiments by schein, et al. and by other groups have periodically found events which showed a large number of high-energy $\gamma^{\prime}$ s which are not accompanied by charged particles. Ruderman and Zwanziger suggested that these events could come from the annihilation of a magnetic monopoleantimonopole pair. T.D. Lee pointed out they could arise from other processes such as the annihilation of high-z lepton pairs.

They propose to use the wide-gap spark chambers which are already set up in the small-angle neutral beam. They will study the forward multigamma production by neutrons and photons in $\mathrm{CH}_{2}$ and $\mathrm{Pb}$ targets. In one beam pulse the target will be exposed to many more particles with energies greater than 100 $\mathrm{GeV}$ than the emulsion stack in which the first "Schein event" was discovered.

The trigger selects events where a neutral particle comes to the target, no charged particles come out, and many charged particles are produced in the lead converter. The wide gap spark chambers should be capable of handing the large number of tracks (up to 100) which may be expected. The proposed experiment is strictly exploratory in nature.

The minimum running time requested is 6 periods of one hour each to tune up the chambers and 4 periods of one shift each for data taking. 


\section{$400 \mathrm{GeV}$ Protons on Complex Nuclei}

D. T. King et al., University of Tennessee

Summary: This proposal requests a nuclear emulsion exposure to 400 $\mathrm{GeV}$ protons. The experiment was actually pursued at $300 \mathrm{GeV}$.

(i) Beam: Protons $300 \mathrm{GeV}$

(ii) Intensity: $1.5 \times 10^{4} / \mathrm{cm}^{2}$ and $3 \times 10^{4} / \mathrm{cm}^{2}$

(iii) Emulsion: Two small Ilford $\mathrm{K} 5$ stacks

(iv) Alignment: Parallel

These observations provide comparison of nuclear evaporation phenomena with similar data taken at $200 \mathrm{GeV}$. The protonnucleus electromagnetic interaction is also examined.

Emulsion processing, scanning: U.T.

Received: July, 1973

Approved: August, 1973

Exposure: October, 1973 


\section{$300 \mathrm{GeV}$ Proton Interactions in Nuclear Emulsion}

J. Hebert, C. J. D. Hebert, H. Areti (Ottawa), M. A. Vincent (Quebec), G. Baumann, R. Devienne (Nancy), J. Lory, C. Meton, D. Schune, B. Willot, B. Charquet, Tsai-Chu, M. Morand (Paris), R Kaiser, J. P. Massue, R. Pfahl, C. Jacquot (Strasbourg), I. Otterlund, (Lund), A. Van Ginneken (Fermilab), J. Juric, o. Adamovic (Belgrade), R. Schmitt (Lyon), J. M. Bolta, J. Pellicier, G. Rey, F. Tejerina (Valencia), F. Fernandez, J. Medena, A. Dura, V. Gardia (Valencia), E. Villars (Santanber).

This study extends the work of Experiment $116(200 \mathrm{GeV}$ protons on emulsion and largely completed) to higher energies. The emphasis is on establishing the general characteristics of inelastic p-nucleus interactions: interaction length, average multiplicity (differentiated by track density), multiplicity and angular distributions, angular and multiplicity correlations, coherent production, etc. Where feasible interactions will be differentiated by target ( $\mathrm{Ag}$ and $\mathrm{Br}$ versus $\mathrm{H}, \mathrm{C}, \mathrm{N}$ and $\mathrm{O}$ ). Results are to be compared with results obtained with emulsion at lower energies, with results obtained with other target elements (including hydrogen), with empirical scaling laws as well as with theoretical predictions.

In the course of these measuremerts special attention will be given to the possibility of ellcountering new (or poorly established) short-lived particles. 
ENGIINESRING RUN FOR TUE 15-FOOT BUBBLE CHAMBER-E-234

D.Bogert, R. Hanft, F.R.Huson, M.Johnson, S.Kahn S.Pruss and W.Smart

This proposal was approved for 50,000 pictures of one sign and one monentum. Without a beam tagging system, the choice was limited to a $\pi$ - or proton beam. We have chosen $\pi$ - as the high energy $\pi$ - beam is unique to FERMILAB.

our data analysis shall commence with the following:

1. Measure all gamma, Vee and kink events to study inclusive neutral distributions and correlations of mul.tiple gamma and Vee events.

2. Measure all 4 prong events on the FERMLAB CRT measuring device SAMM. This will give diffractive crois sections. Also with the high statistics available various Pomeron models can be tested and $\pi$ in scattering can be studied.

3. Scanising and measuring of a sample of the film for an inclusive study of charged prongs. 


\section{A PROPOSAL TO EXPLORE THE LARGE-P DOMAIN: INCLUSIVE CROSS SECTIONS AND POSSIBLE JET STRUCTURE}

V. Cook, G. Hicks, P.M. Mockett, W.P. Oliver, J.E. Rothberg, R.W. Williams, K.K. Young (Univ. of Washington); P. Limon, P. Mantsch, R. Orr, S. Pruss (National Accelerator Laboratory)

This experiment plans to achieve the following goals:

i) High statistics measurements of the single particle inclusive cross sections above $\mathrm{P}_{\mathrm{t}}=2 \mathrm{GeV} / \mathrm{c}$ produced by $\pi^{ \pm}, \mathrm{K}^{ \pm}$and $\mathrm{p}^{ \pm}$.

ii) Search for hadron jets associated with the large-Pt events. The momentum of each charged particle in the jet will be measured so that a full correlation study will be possible. In a large fraction of cases the outgoing species will also be identified by three

Cerenkov counters.

iii) Observe the recoil structure opposite the large- $P_{t}$ particle (or jet) with a photon and hadron calorimeter subtending about 2.7 steradians.

iv) Observe the rapidity distribution for all charged particles accompanying the large- $\mathrm{P}_{\mathrm{t}}$ event.

This experiment will run in beam Ml-East of the Meson Area following Experiment \#7. It utilizes a large aperture magnetic spectrometer ( 1 sr aperture) followed by three segmented threshold Cerenkov counters. This is designed to study in detail the charged particle component of large $\mathrm{P}_{t}$ events. Three hodoscope arrays are employed to select events where at least one charged particle has a $P_{t}>1.5 \mathrm{GeV} / \mathrm{c}$. Particle species will be identified over the full kinematic range of the spectrometer. The spectrometer will explore the center of mass angular region of $45^{\circ}$ to $110^{\circ}$.

The spectrometer arm will be complemented by a photon and hadron calorimeter on the recoil side. This will cover an angular range of $60^{\circ}$ to $135^{\circ}$ in the center of mass. The calorimeter will be segmented so that a trigger on large $P_{t}$ can be employed. It will allow the study of jet-jet correlations as well as a measurement of the neutral component of large-P $t$ events.

The experiment will utilize a 13,000 wire MWPC system to allow high data rates with good duty cycle. PWPC's will also give high efficiency for the large multiplicity events expected in this domain.

Employing a 12-inch $\mathrm{LH}_{2}$ target and $5 \times 10^{6}$ incident particles per pulse at $200 \mathrm{GeV} / \mathrm{c}$, the experiment will obtain $\sim 2 \times 10^{5}$ events with $\mathrm{P}_{t} \geq 3$ $\mathrm{GeV} / \mathrm{c}$ from the spectrometer trigger and $\sim 10^{6}$ from the calorimeter trigger per 100 hours of data-taking.

In 500 hours of data taking the experiment is expected to cover three beam momenta of both sign and all the six types of beam particles. It will explore how $P_{t}$ is balanced in large- $P_{t}$ events and $100 k$ for the predicted jet-like structure associated with large- $P_{t}$. 
EMULSION EXPOSURE TO $300 \mathrm{GeV}$ PROTONS

J. J. Lord (University of Washington)

Proposed Continuation and Extension at $300 \mathrm{GeV}$.

1. The first phase of our experiment \#17l was carried out at $200 \mathrm{GeV}$ in Sept. 1972. The purpose of the experiment was the search foi new shcrt-lived particles produced in nuclear collisions in emulsion.

2. We would like to extend our search for snort-livec particles to $300 \mathrm{GeV}$ in case there is a higher threshold energy for their production.

3. A side-line found during the $200 \mathrm{GeV}$ experiment was the study of intranuclear cascades in proton-tungsten nuclear collisions. We would like to extend this to collisions with other nuclei from $Z=5$ to $Z=90$ in order to compare with the Independent Particle Model and the coherent Particle Model theories of J.S. Trefil. A portion of this experiment could be carried out at energies of either or both $200 \mathrm{GeV}$ and $300 \mathrm{GeV}$.

4. The experiment can be carried out vith $\epsilon$ separate packets of plates each $2 " \times 6 "$ and 10 " long.

5. An exposure of abcut 75,000 to 200,000 protons per square centimeter would be needed.

Summary prepared by

J. J. Lord, August 1973 
EMULSION EXPOSURE TO $400 \mathrm{GeV}$ PROTONS

J. J. Lord (University of Washington)

Proposed Continuation and Extension at $400 \mathrm{GeV}$

1. The first phase of our experiment 171 was carried out at $200 \mathrm{GeV}$ in Sept. 1972. The purpose of the experinent was the search for new short-livea particles produced in nuclear collisions in emulsion.

2. We would like to extend our search for short-lived particles to $\leq 00 \mathrm{GeV}$ in case there is a higher threshold energy for their production.

3. A side-line found during the $200 \mathrm{GeV}$ experiment was the stucy of intranuclear cascades in proton-tungsten nuclear collisions. We would like to extend this to collisions with other nuclei from $z=5$ to $z=90$ in orcer to compare with the Independent Farticle Model and the Coherent Particle Model theories of J.S. Trefil. A portion of this experiment could be carried out at $400 \mathrm{GeV}$.

4. The experiment can be carried out with 6 separate packets of plates each 2" $x$ " and 10" long.

5. An exposure of about 75,000 to 200,000 protons per square centimeter would be needed.

Summary prepared by

J. J. Lord, August 1973 
PROPOSAL FOR A FURTHER SEARCH FOR LONG-IIVEI PARTICIES AT NAL

S. Frankel, W. Frati, L. Resvanis, W. Yang

(Univ. of Pennsylvania); F. Nezrick (NAL)

The recently completed experiment 199 was sensitive to long-lived particles produced by 300 and $400 \mathrm{BeV}$ protons if their lifetimes were in the range of 1 to 1000 hours. The upper limit on their production and capture cross-section was less than $10^{-36} \mathrm{~cm}^{2}$.

The authors have just completed taking data which pushed this search down to particles with mean lives in the 10 millisecond to 10 hour region. This was accomplished by looking between spills for ultrarelativistic decay particles, with a gas Cerenkov counter aimed directly at the target, at $90^{\circ}$ with respect to the incoming beam. Preliminary analysis shows an upper limit of $10^{-36} \mathrm{~cm}^{2}$ for production and capture of particles with mean lives between 10 milliseconds and 10 hours.

Summary reviewed sy

W. Frati, iebruery 1976 


\author{
STUDY OF SECONDARY PARTICLES PRODLCED BY $300 \mathrm{GEV}$ \\ PROTONS IN EMULSION CHAMBERS. \\ K. Hoshino, S. Kuramata, K.Niu, K.Niwa, S.Tasaka \\ and Y.Yanagisawa (Nagoya University) \\ Y.Maeda (Yokohama National University) \\ N. L'shida (Aichi Educational University)
}

SUMMARY OF PROPOSAL

The purposes of this exposure are 1. Calibration of relative scattering method for determination of momentum of charged particles. 2. The distribution of energy given to $Y$ rays and the distribution of elasticity of collisions. 3. Search for short-lived particles as found by author in an enulsion chamber exposed to cosmic rays. Tro emulsion chamber consisting of nuclear emulsions and thin lead plates the size of which $1 \mathrm{~s} 12 \mathrm{~cm} \times 9.5 \mathrm{~cm} \times 7 \mathrm{~cm}$ will be exposed.

\title{
STATUS
}

COMPLETED: October, 1973

EXPERIMFNTAL RESULT AND PUBLICATIONS.

1) APPLICATION OF THE RELATIVE SCATIERING METHOD TO THE ANALYSIS OF SUPER HIGH ENERGY JET SHOWERS.

K. Hoshino et al.

PROCEEDINGS OF INTERNATIONAL COSHIC RAY SYNPOSIUM ON HIGH FNFPGY PHENOMENA. COSMIC RAY LABORATORY, UNIVERSITY OF TOKYO. MOVEILLR 1974 , p. 155

Calibrations of the relative scattering method for determination of momentum of charged particles were carried out exposing emulsion chambers to protons of $303 \mathrm{GeV} / \mathrm{c}$ at the FNAL and to negative $\pi$ mesons of $8 \mathrm{GeV} / \mathrm{c}$ at the ANL. 


\author{
STUDY OF SECONDARY PARTICLES PRODUCED BY $400 \mathrm{GEV}$ \\ PROTONS IN EMTLSION CHAMBERS. \\ K. Hoshino, S.Kuramata, K.Niu, K.Niwa, S.Tasaha \\ and Y.Yanagisawa (Nagoya University) \\ Y.Maeda (Yokohama National iniversity) \\ N.Ushida (Aichi Educational University)
}

\title{
SUMMARY OF PROPOSAL
}

The purposes of this exposure are 1 . Search for short-lived particles as found by author in an emulsion chamber exposed to cosmic rays. 2. The distribution of energy given to $\gamma$ rays and the distribution of elasticity of collisions. 3. Calibration of relative scattering method for determination of moment um of charged particles.

Several emulsion chambers consisting of nuclear emulsions and thin low and high $Z$ materials the size of which is $12 \mathrm{~cm} \times 9.5 \mathrm{~cm} \times 7 \mathrm{~cm}$ will be exposed.

\section{STATUS}

COMPLETED: December, 1975

Exposed emulsion chambers are now on the scanning and analysing stages at each member university. 


\section{STUDY OF $300 \mathrm{GeV}$ PROTON INTERACTION WITH NUCLEAR EMULSION}

Piyare L. Jain (State Univ. of New York at Buffalo)

In $300 \mathrm{GeV}$ proton interacting with nuclear emulsion, we are interested in the study of 1) white stars as was done 1 at $200 \mathrm{GeV}, 2$ ) coherent production of particles, 3) characteristics of multiplicity distribution, 4) electron-pair production, 5) study of those events which interact with complex nuclei $\left(\mathrm{N}_{\mathrm{L}} \geq 2\right)$, the multiplicity of their shower tracks as a function of $\left.\mathrm{N}_{h}, 6\right)$ behavior of backward particles for which momenta and masses are calculated.

We shall expose a small stack of 20-25 pellicles of size $10 \times 20 \times 600$ thick to a proton beam with a flux density $\sim 5 \times 10^{4}$ particles $/ \mathrm{cm}^{2}$. We shall collect the data by scanning along the track and by area scanning.

1. Coherent production of particles by $200 \mathrm{GeV} / \mathrm{c}$ protons in Nuclear Emulsion, N.C. Lett (1974).

2. Correlation of particles at $200 \mathrm{GeV}$ proton-proton $\rightarrow$ interaction, N.C. Lett (1974)

3. International Conference on New Results from Experiments on High Energy Particle Collisions, Vanderbilt, Tenn. (1973).

4. Electron pair production by $200 \mathrm{GeV}$ proton in nuclear emulsion ADS meeting, Washington, D.C. Apine 1974 . 


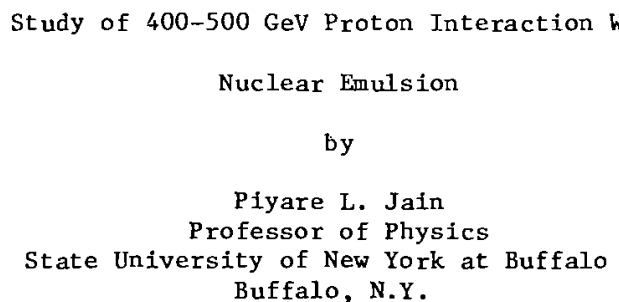

In 400-500 Ge V proton interacting with nuclear emulsion, we are interested in the study of (i, white stars as was done ${ }^{1}$ at $200 \mathrm{GeV}$, (ii, coherent production of particles, (iii, characteristics of multiplicity distribution, (iv, electron-pair production, (v, study of those events which interact with complex nuclei $\left(\mathrm{N}_{2} \geq 2\right)$, the multiplicity of their shower tracks as a function of $\mathrm{N}_{h}$, (vi, behavior of backward particles for which momenta and masses are calculated.

We shall expose a small stack of $20-25$ pellicles of size $10 \times 20 \times 600$ thick to a proton beam with a flux density $\sim 5 \times 10^{4}$ particles/cm ${ }^{2}$. We shall collect the data by scanning along the track and by area scanning.

1. Coherent production of particles by $200 \mathrm{GeV} / \mathrm{c}$ protons in Nuclear Emulsion, N.C. Lett (1974).

2. Correlation of particles at $200 \mathrm{GeV}$ proton-proton $\rightarrow$ interaction, N.C. Lett (1974)

3. International Conference on New Results from Experiments on High Energy Particle Collisions, Vanderbilt, Tenn. (1973).

4. Electron pair production by $200 \mathrm{GeV}$ proton in nuclear emulsion Aps meeting, Washington, D.C. Apine 1974. 


\section{SEARCH FOR DIFFERENCE IN PION/PROTON INTERNAL STRUCTURE}

W. Selove, L. Kroger, T. Kondo, W. Kononeko, E.M. O'Neil (University of Pennsylvania); A.R. Erwin, E. Harvey, R. Loveless, M. Thompson (University of Wisconsin)

Bjorken et al. suggested that parton-parton collisions might produce jet pairs at large angles, separable from jets along the beam direction. If found, these jet pairs could give direct evidence of parton-parton collisions and of the internal momentum distribution of partons in hadrons. Because the pion is made of fewer components (2 quarks) than the proton ( 3 quarks) the probability for high-momentum components inside the pion should be greater than for high-momentum components inside the proton. This effect would cause the products of a parton-parton collision from a $\pi p$ initial state to exhibit a forward-backward asymmetry in the over $\pi p$ c.m. system (resulting from the 2 quark versus 3 quark collision). This asymmetry should be quite large in high-p $p_{t}$ events.

They propose to study jet-pairs in $\pi p$ and pp collisions with the following objectives:

i) To look for an asymmetry in the production of forward pairs compared to backward pairs;

ii) To measure the ratio of event rates with jets of given $\mathrm{p}_{\mathrm{t}}$ compared to single particles of the same $\mathrm{p}_{\mathrm{t}}$.

iii) To study the distribution of multiplicities in jets and correlations in these multiplicities for jet pairs.

The detector chosen is a calorimeter hodoscope. A pair of calorimeters on the two sides of the beam is proposed to detect typical jets of 3 particles with total energy of $60 \mathrm{GeV}$ and angular separation of 10-20 mrad. Each calorimeter will be made of about 20 elements, each with a diameter of a cascade shower (about 8" to 16"). The calorimeters will be located about 20 meters from the target and should give $\delta \mathrm{E} / \mathrm{E} \sim 10 \%$ at $50 \mathrm{GeV} / \mathrm{c}$. The angular resolution of the hodoscopes will be $\delta \theta \sim$ few mrad.

They estimate counting rates of about $30 \pi^{\prime} \mathrm{s}$ per hour in each hodoscope with $p_{t}>6 \mathrm{GeV} / \mathrm{c}$ and coincidence rates of about 1 per hour, assuming 109 negative pions per burst. The beam momentum spread could be $\pm 5 \%$ and have an angular divergence of a few mrad. If $10^{9}$ pions per burst cannot be achieved, they will lower the transverse momentum requirement. 


\section{A PROPOSED EXPERIMENT TO SEARCH FOR CHARMED PARTICLES AND HEAVY LEPTONS}

Universities of Brussels, Dublin, London, Rome, Strasborg and the Fermi National Accelerator Laboratory

E.H.S. Burhop (Spokesman)

If charmed particles and/or heavy leptons exist and have a mass in the range $1-3 \mathrm{GeV}$ it appears that their mean life is likely to lie in the range $3 \times 10^{-12}-3 \times 10^{-15}$ sec. This lifetime is outside that which could be directly detected in bubble chambers and in electronic detectors; it is instead within the capabilities of the photographic emulsion technique.

It is proposed to search for such short-lived particles produced in neutrino interactions, with a hybrid emulsionspark chamber arrangement. Three scintillation counters would trigger two optical spark chambers; the neutrino interaction in emulsion can be readily located by following back into the emulsion the tracks of the spark chamber. Such a system proved to be workable at CERN.

The experimenters aim to have about 1000 neutrino interactions in a 20 litre emulsion stack.

Summary reviewed by

E.H.S. Burhop, January 1976 


\section{NEUTRON PROTON DIFFRACTION SCATTERING}

C. DeHaven, C. Ayre, T. Roberts, P.V.R. Murthy, R. Gustafson, I.W. Jones, M. Longo, J. Pluta, J. Stone, J. Chanowski (University of Michigan)

In this experiment it is planned to measure differential cross sections for $\mathrm{np}$ scattering in the diffraction region $\left[0.1<t<3.5(\mathrm{GeV} / \mathrm{c})^{2}\right]$ in the energy range 50 to $400 \mathrm{GeV}$. The small-angle neutron beam will be used. The apparatus will be set, up in and downstream of the detector building. Forwardgoing neutrons will be detected in an array of wire spark chambers interspersed with steel plates. [This array has already been used in a similar experiment at Argonne.] The recoil protons coming off near $90^{\circ}$ in the lab will be detected in a wire spark chamber spectrometer. The incident neutron energy for each event can be reconstructed (to $\approx 5 \%$ accuracy) from the measured angle of the scattered neutron and the proton angle and momentum.

The precision of the differential cross section results should permit determination of the slope of the diffraction peak to a few percent, should be sensitive to changes in slope with $s$ and $t$ of the magnitudes expected in pp elastic scattering, and should permit study of the possible development with $s$ of a diffraction minimum and secondary maximum (as seen in the ISR pp data). 


\section{CRACOW EMULSION EXPOSURE TO $400 \mathrm{GeV}$ PROTONS}

W. Wolter (Inst. of Nuclear Physics, Krakow, Poland)

There is at present a widespread opinion $1,2,3$ that the multiple production in hadron-nucleus collisions at high energies provides a sensitive test for the mechanism of multiple production in hadron-nucleon collision. Our previcus investigations performed in nuclear emulsion exposed to $200 \mathrm{GeV}$ protons at NAL 4,5 have shown that photographic emulsion method offers a unique possibility of selecting events according to the actually realized number of collisions inside the nucleus. This is due to the fact that in nuclear emulsion we know what happens with the hit target nucleus through the direct observation of the number of heavily ionizing particles $\mathrm{N}_{\mathrm{h}}$ which are the products of spallation or evaporation of the nucleus.

In the proposed experiment we would like to extend our observations of regularities in rapidity distributions as a function of the target nucleus excitation 5 , to higher energies. Therefore we ask for the exposure of 5 stacks of IIFORD G-5 stript emulsion to $400 \mathrm{GeV}$ proton beam at NAL. Emulsions will be prepared in plastic containers (about $5 \mathrm{~cm} \times 5 \mathrm{~cm} \times 10 \mathrm{~cm}$ ) with an engraved straight line which will be used for optical adjusting of the stack parallel to the beam $( \pm 10 \mathrm{mrad})$. The optimum density of proton tracks in the stack is $10^{5}$ to $3 \times 10^{5}$ tracks $/ \mathrm{cm}^{2}$.

Within 6 months after the exposure we expect to analyze about 1000 interactions in our laboratory in Cracow.

\section{References}

1. A. Dar, J. Vary, Phys. Rev. D6, 2412 (1972)

2. P.M. Fishbane, J.S. Trefil, Phys. Rev. Lett. 31, 734 (1973)

3. K. Gottfried, Ref. TH. 1735-CERN

4. J. Babecki et al., Phys. Lett. B47, 268(1973)

5. J. Babecki et al., INP Report 854/PH Cracow 1973; to be published in Acta Fhysica Polonica B, 1974. 
PHENOMENOLOGICAL STUDY OF PROTON-NUCLEUS COLLISIONS AT $300 \mathrm{GeV} / \mathrm{C}$

IN EMULSION

O.Kusumoto, S.Ozaki and M.Teranaka (Osaka City University); G.Fujioka, H.Fukushima, K. Kobayakawa, M.Miyagaki and Y.Tsuzuki (Kobe University); H.Okabe and J.Yokota (Science Education Institute of Osaka Prefecture); M.Ohta (Kinki University).

Summary:

The first purpose of the experiment is to derive the mean free paths of the coherent productions in emulsion at $300 \mathrm{GeV} / \mathrm{c}$ and to derive the energy dependence of these processes by comparing with the results so far obtained at lower energies. The second purpose is to derive the mass number dependence of the cross section for the inelastic proton-nucleus collision at this energy.

Procedure:

A stack of $\mathrm{K} 5$ pellicles of $3^{\prime \prime} \times 6^{\prime \prime} \times 600 \mu \mathrm{m}, 24$ sheets, is exposed to the proton beam with a density of $2 \times 10^{4}$ protons $/ \mathrm{cm}^{2}$. The coherent events are detected using along-the-track scanning and generally accepted criteria. 


\section{PHENOAENOLOGICAI STUDY OF PROTON-NUCLEUS COLLISION}

\section{A $400 \mathrm{GeV} / \mathrm{C}$ IN ENULSION}

S. Ozaki, S. Higashi, O. Kusuroto and M. Teranaka (Osake City University); G. Fujioka, H. Fukushime, K. Kobayakawa, K. Miyagaki and Y. Tsuzuki (Kobe Lniversity); $M$. Ohta (Kinki University)

\section{Summary:}

Main aim of this experiment is to study the following features of the proton-nucleus collision in nuclear emulsion at $400 \mathrm{GeV} / \mathrm{c}$.

1) Mass number dependence of the cross section for the inelastic protonnucleus collision.

2) Energy dependence of the cross section for the coherent production.

3) Distributions of multiplicity and of emitted angles with fast charged particles produced.

4) Search for any short-lived particle.

1) and 3) are stidied as a relating problem to the recent result with small mean rultiplicity of fast charged particies produced in tie hïgh energay protonnucleus collision ${ }^{1}$ which may open a new way to study elementary processes.

2) is the study of the coherent production in which the enrgy dependence of its cross section is the main problem in this analysis. Any short-lived particle with a life between $10^{-12}$ and $10^{-16} \mathrm{sec}$ is searched during the course of the measurement 3 ). This experiment is made as an extension of the experiments at $200 \mathrm{GeV}$ ("III) and at $300 \mathrm{GeV}(\$ 250)^{2}$.

1. L. Van Hove, Th. 1746-CERN (1973); K. Gottfried, Th. 1735-CERN (1973)

2. The results obtained in the experiment \#117 on the problems 1) and 2) mentiond above is submitted to the Physical Review Ietters. The analysis of the experiment $\$ 250$ is now going on.

\section{Procedure:}

An ordinary stack which consists of 24 sheets of pellicles of nuclear emulsion, each having a size of $3^{\prime \prime} \times 6^{\prime \prime} \times 600$ micron, is exposed to the 400 $\mathrm{GeV} / \mathrm{c}$ proton beam with a density around $10^{4}$ protons per $\mathrm{cm}^{2}$. The events induced by the proton-nucleus collision in emulsion are detected by alongthe-track scanning. It is scheduled that the main part of the analyses will be finished within a year. 


\section{STUDY OF MUL'RIPARTICLE PRODUCTION}

IN A 30" BUBBLE CIIAYBER

J. Chapman, M. Green, B. Roe, D. Sinclair, J. Vandervelce, (Michigan); C. Bromberg, D. Cohen, T. Ferbel, P. Slattery, D. Underwood (Rochester)

The Michigan and Rochester bubble chamber groups are proposing a collaborative investigation of pp interactions at high energy. This proposal is for a general survey using the bare hydrogen bubble chamber. The request is for 50,000 pictures with a proton beam at each of two energies - $100 \mathrm{GeV} / \mathrm{C}$ and $400 \mathrm{GeV} / \mathrm{c}$.

The purpose is to extract the energy dependence of the total cross section and of multiparticle production processes; to measure such energy dependence, the analysis must be carried out using uniform scanning rules and normalization techniques, consequently, Michigan and Rochester will in effect function as a single group. In addition to looking for unusual events by means of a very rapid scan by physicists, the intent is also to measure all topologies and test current theoretical models of hadronic interactions. 
NEUTRINO-ELECTRON SCATTERING AT NAL

H.L. Anderson, L.W. Mo, S.C. Wright (Univ. of Chicago); N.E. Booth (Univ. of Oxford)

It is proposed to measure the cross sections for the reaction $v_{\mu} e^{-}+v_{\mu} e^{-}$and eventually for the antineutrino counterpart. These reactions and the $v_{e} e^{-\rightarrow v} e^{e^{-}}$reaction have not yet been observed experimentally. Their measurements will answer the following questions:

1. The existence of lepton-lepton current interactions.

2. The distinction between various weak interaction theories such as:

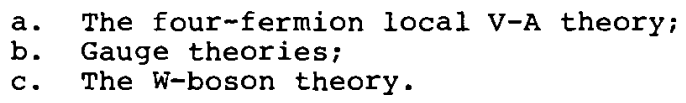

3. The existence of a lepton neutral current.

The ve interactions will be detected in a 50-cell calorimeter, $1 \mathrm{~m}^{2}$ in area. Each cell is made of one multiwire proportional chamber (MWPC) with a $2 \mathrm{~cm}$ aluminum plate support and $1.38 \mathrm{~cm}$ of steel ( $1 \mathrm{r.1.}$ ). A few steel plates may be replaced with lead-glass.

The anode signals from the MWPC will be used to trigger the system, when the signals from several adjacent chambers show the characteristics of an electron shower.

Assuming a cross section

$$
\sigma\left(E_{\nu}\right)=10^{-41} \mathrm{E}_{\nu}(\mathrm{GeV}) \mathrm{cm}^{2}
$$

they should obtain 137 events in 1000 hours of running with $10^{13}$ protons of $350 \mathrm{GeV}$ on the primary target. These events should be adequate for distinguishing among the various theories. They propose to set up the equipment behind Experiment \#IA and to run in parallel with other neutrino experiments under any beam conditions. 
PROPOSAL TO SEARCH FOR A SECOND MUON NEUTRINO, $\lambda_{\mu}$ G.R. Kalbfleisch, (BNL); E.C. Fowler (Purdue) and NAL-Caltech Group (Exp. \#2IA)

It is proposed to check experimentally whether the neutrino from kaon decay $\left(\mathrm{K}^{+} \rightarrow \mu^{+}+\lambda_{\mu}\right)$ is different from the neutrino from pion decay $\left(\pi+\rightarrow \mu+\nu_{\mu}\right)$. If $\lambda_{\mu} \neq \nu_{\mu}$ then it is probably that the
ratio

$$
R=\frac{\sigma\left(\lambda_{\mu} N\right)}{\sigma\left(\nu_{\mu} N\right)}
$$

of the interaction cross section of the neutrinos with nucleons at the same energy, E, differs from unity. Some indications in this direction may already exist in the Gargamelle bubble chamber neutrino experiment at CERN.

They propose to use the Caltech apparatus of Experiment \#2IA in the NAL narrow-band neutrino beam. Assuming a value of $R$ close to unity, $R$ may be measured to a $\pm 10 \%$ precision at neutrino energies around 50-70 GeV, For this purpose they request $3 \times 10^{17}$ protons onto the primary target. The running will be done at two different charged particle momenta, in order to have one overlapping energy for both $\lambda_{\mu}$ and $\nu_{\mu}$. The accuracy in the determination of the number of neutrinos will be assured by monitoring the muon flux in the shielding as well as the protons on target and the pions and kaons decaying in the vacuum pipe.

The experiment could later be extended to $10^{18}$ protons ( 1500 events), in order to reach a precision of $\pm 5 \%$, and to two different neutrino momenta. 
INTERACTION OF $150 \mathrm{GEV}$ MUONS WITH EMULSION NUCLEI

Piyare L. Jain (State Univ. of New York at Buffalo)

Recently we have reported the direct-electron-pair production from the coulomb field of an emulsion nucleus by 200 $\mathrm{GeV}$ protons ${ }^{1}$ from the Fermi Iraboratory and $15.8 \mathrm{GeV} / \mathrm{c}$ muons 3 from the Brookhaven National Laboratory, in order to test the predictions of the theories based on quantum electrodynamics at small distances. From most of the present theories $4-7$, the predictions for electron production cross section depends on the ratio $Y(=E / m)$ and not on $E$ and $m$ separately or on the type of the incoming particle. Our recent results indicates that the mass of the incoming particle is perhaps playing an

important role. In order to check this point we woule like to have two or three more muon beam exposures between $150 \mathrm{GeV}$ at FNAL. These experiments will help us to settle the mass dependence question very clearly.

References

1. P.L. Jain, M. Kazuno, B. Girard, and z. Ahmad, Phys. Rev. Lett. $32,797(1974)$.

2. P.I. Jain, M. Kazuno, and B. Girard, Phys. Rev. Lett. 32 , $1400(1974)$.

3. P.I. Jain, M. Kazuno, and B. Girard, Phys. Rev. (accepted Feb. 1975).

4. H.J. Bhabha, Proc. Cambricge Pril. Soc. 31, 394 (1935) and Procd. Roy. Soc. A, 152, 559 (1935).

5. G. Racah, N.C. 14, 93 (1937).

6. T. Murota, A. Weda and H. Tanada, Progress Theor. Physics, 16. $482(1956)$.

7. M.M. Block, D.T. King and W.W. Wada, Phys. Rev. 96, 1627 (1954). 


\section{A PROPOSAL TO MEASURE PARTICLES PRODUCED AT HIGH TRANSVERSE MOMENTUM BY PIONS}

J.w. Cronin, H.J. Frisch, M.J. Shochet (Univ. of Chicago); J.P. Boymond, P.A. Piroué, R.L. Sumner (Princeton Univ.)

It is proposed to measure single particle inclusive spectra at high transverse momenta for negative pions incident on a heavy nuclear target (and also hydrogen if possible).

$$
\begin{aligned}
\pi^{-}+\mathrm{Z} & +\pi^{ \pm}+\text {anything } \\
& \rightarrow \mathrm{K}^{ \pm}+\text {anything } \\
& +\mathrm{p}^{ \pm}+\text {anything } \\
& \rightarrow \mu^{ \pm}+\text {anything }
\end{aligned}
$$

The main purpose of the experiment is the comparison of particle production at large transverse momenta in p-nucleus and $\pi^{-}$-nucleus collisions. If quarks or partons play a role in large $p_{t}$ phenomena, it would seem that replacing incident protons by pions should lead to significant differences in the ratios of secondaries produced. Also direct muon production might be enhanced because of the definite anti-quark cortent of a pion beam.

They propose to build a high intensity pion beam, using a quadrupole triplet and one bending magnet. Such an arrapgement should allow the production of a $200 \mathrm{GeV} / \mathrm{c}$ beam of 10 lf $\pi$ per $10^{13}$ interacting protons of $400 \mathrm{GeV} / \mathrm{c}$. The momentum bite of the $\pi^{-}$beam would be $\Delta \mathrm{p} / \mathrm{p} \simeq \pm 4 \%$.

For the detection of the reactions above they will use the same heavy targets and the same spectrometer presently employed in Experiment \#100A for studying charged particle production by protons at $\theta=80 \mathrm{mrad}$. The expected rates should allow observation of particle production up to $p_{t} \simeq 6 \mathrm{GeV} / \mathrm{c}$. 


\section{A PROPOSAL TO STUDY HIGH P PHYSICS WITH A MULTIPARTICLE SPECTROMETER}

G. Fox, R. Gomez, J. Pine, C. Bromberg (Caltech); E. Lorenz, P. Schlein (UCLA); R. Abrams, H. Goldberg, S. Margulies, D. McLeod, J. Solomon (University of Illinois, Chicago Circle); A. Dzierba (Univ. of Indiana); v. Ashford, R. Juhala, E. Malamud, S. Mori (Fermilab)

It is proposed to use the multiparticle spectrometer system, now being constructed for Experiment \#110A, to gain a global view of interactions in which one or more particles carry off high values of $\mathrm{p}_{\mathrm{T}}$.

Recent experiments at the CERN-ISR and at NAL have shown that the expotential transverse momentum distribution for single particle spectra apparently becomes a power law distribution at high values of $\mathrm{p}_{\mathrm{T}}$. The interest in these results has been intensified by the interpretation that in high transverse momentum particles are the result of hard parton-parton collisions. While the parton picture is qualitatively appealing, the theory is in no sense detailed and there is also the possibility that the non-parton picture is more correct. It is thus very interesting to look at the large $\mathrm{P}_{\mathrm{T}}$ phenomena in a more detailed way, in particular to see what the entire interaction looks like when one of these high $\mathrm{P}_{T}$ particles is emitted.

The multiparticle spectrometer of Experiment \#I10A will be complemented by three calorimeters for both photons and hadrons. Two will be on either side of the beam line, covering c.m. angles from about 60 to 120 degrees, each subtending about 90 degrees in azimuth. The third calorimeter, for more forward directions, spans the c.m. angular range from about 15 to 50 degrees, with almost complete azimuthal coverage. The $\mathrm{p}_{\mathrm{T}}$ for charged particles will be determined with the spectrometer, the calorimeters being used merely to generate a high $\mathrm{P}_{\mathrm{T}}$ trigger. For photons and neutrons the calorimeters will provide the $\mathrm{p}_{\mathrm{T}}$ determination.

The spectrometer will give $4 \pi$ acceptance for detection of charged particles, and large solid angle for momentum measurement and particle identification. Several triggers are discussed, but the main emphasis is on the calorimeters, which detect high $\mathrm{p}_{\mathrm{T}}$ particles, or groups of particles, over a large part of the c.m. angular range from 0 to 120 degrees.

The apparatus is expected to give detailed information in range $2<\mathrm{pq}_{\mathrm{T}}<6 \mathrm{GeV} / \mathrm{c}$. They request $100-150$ hours at $200 \mathrm{GeV} / \mathrm{c}$ for a first test. To pursue the high $\mathrm{P}_{T}$ study further, they will require 300-500 hours. The running for this experiment may be interleaved with the running for Experiment \#IIOA. 


\section{PROPOSAL TO TEST TRANSITION COUNTERS AT NAL}

J. Fischer, S. Iwata, V. Radeka, C.I. Wang (Brookhaven National Laboratory)

A test is proposed of transition radiation counters to differentiate pions from kaons at NAL energies. One proposed arrangement involves radiation foils with a NaI transition radiation detector downstream of a magnet used to deflect away the beam. Another scheme is a counter comprised of an array of wire proportional chambers and radiator foils without such a deflecting magnet.

It is proposed to place this detector in the $M 6$ beam in the Meson Area to be run parasitically with Experiment \#69A. Total running time is estimated to be 200 hours.

Summary reviewed by

C.L. Wang, February 1974 
NEUTRAL CURRENT INVESTIGATIONS AT NAL

\section{B. C. Barish and F. Sciulli (Caltech)}

They propose to run the equipment of experiment $21 \mathrm{~A}$ in the narrow band neutrino beam for a verification of the recently reported neutral currents. The ratios of neutral-to-charged current contributions is at present:

$$
\begin{aligned}
& R=\frac{\sigma\left(\nu_{\mu}+\mathrm{N} \rightarrow \nu_{\mu}+\text { hadrons }\right)}{\sigma\left(\nu_{\mu}+\mathrm{N} \rightarrow \mu^{1}+\text { hadrons }\right)}=0.20 \pm 0.03
\end{aligned}
$$

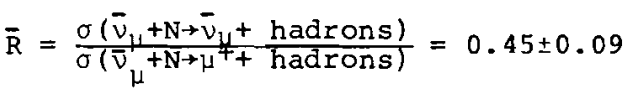

The major background in the Gargamelle bubble chamber experiment at CERN was due to neutron interactions, while in the experiment IA at NAL it consisted of the small efficiency for observing low energy muons.

The proposed experiment does not have any background arising from neutrons and minimizes the misidentifications of muons. This last point comes about because

i) The neutrinos are of high energy; thus there are no low energy muons, which go at large angles and thus may go undetected. Moreover the background coming from the wide angle component of neutrinos can be measured by increasing its contribution in a separate run.

ii) The experiment is proposed for the antineutrino beam, which has a more favorable situation than the neutrino case because of the larger fraction of energy which goes to the muon and because $\bar{R}$ seems to be larger than $R$.

iii) The calorimeter of experiment $21 \mathrm{~A}$ has a good acceptance for muons.

They request a total of $3 \times 10^{17}$ protons on target at an average intensity of $2 \times 1012$ protons/pulse with fast spill.

Summary reviewed by

B. Barish/F. Sciulli, February 1974 
A PROPOSAL TO STUDY PHOTOPRODUCTION OF $\phi$ MESONS

U. Becker, J. Burger, M. Chen, J. Leong, S.I. Wu, S.C.C. Ting (MIT); T. Nash, R.F. Stiening (Fermilab); E. Coleman, E. Marshak (Univ. of Minnesota)

It is proposed to measure the differential cross section for $\phi$ mesons and search for new narrow resonances produced in the reactions

$$
\begin{aligned}
& \gamma+\mathrm{p} \rightarrow \phi+\mathrm{p} \rightarrow \mathrm{K}^{+} \mathrm{K}^{-}+\mathrm{p} \quad \text { (1) } \\
& \gamma+p+\text { narrow resonances }+x \\
& \text { + multiple hadrons and/or electromagnetic } \\
& \text { particles (2) } \\
& \text { and } \gamma+\mathrm{A} \rightarrow \ell^{+}+\ell^{-}+\mathrm{A} \text { where } \ell^{ \pm} \text {represents high mass leptons }
\end{aligned}
$$

in the 20 to $195 \mathrm{GeV}$ range using a tagged photon beam.

Reaction ( 1 ) is the best approximation to a pure Pomeron exchange reaction. Interesting features versus energy are:

a. From the observed increase in the $\mathrm{K}^{+} \mathrm{p}$ and $\mathrm{pp}$ total cross sections one expects that the forward differential cross section for (1) to increase by about $40 \%$ in the above energy range.

b. At low energies the slope of the forward peak in reaction (1) is measured to be $b \simeq 4(\mathrm{GeV} / \mathrm{c})-2$, contrary to most other reactions where it is $b \simeq 8-10$ $(\mathrm{GeV} / \mathrm{c})-2$.

c. No structure has been observed in the angular distributions of (1), again contrary to the scattering of other particles.

The equipment for measuring reaction (1) will consist of three sets of chambers for detection of the recoil protons. Two standard magnets (of the BM109 type) with opposite magnetic fields bend the forward-going particles horizontally, refocusing particles with the same production angle at a point 120' downstream of the target. Four proportional chambers and two drift chambers measure the momenta of the forward-going particles. Two multiple tube Cerenkov counters distinguish $\pi, K, p$ up to $120 \mathrm{GeV}$ and can detect a maximum of 16 final state charged particles. With shower counters in the back one can also detect electromegnetic particles such as e, $\gamma, \pi^{\circ}, \eta^{\circ}$, etc.

With 480 hours of running with $e^{-}$at $200 \mathrm{GeV}$ and 120 hours with $e^{-}$at 160 $\mathrm{GeV}$, we expect to obtain $10^{5}$ events of reaction (1), covering the range 20 $<\mathrm{k}<195 \mathrm{GeV}$ and $0<|\mathrm{t}|<2(\mathrm{GeV} / \mathrm{c})^{2}$. The background is expected to be negligible.

The same apparatus as proposed to detect $\phi+\mathrm{K}^{+} \mathrm{K}^{-}$can also detect narrow resonances and high mass lepton pairs - similar to the $J$ particle recently found at BNL and Spear and the mysterious je events found at spear - decaying into hadronic as well as leptonic final states.

We expect $\sim 50 \mathrm{~J} \rightarrow$ ee/day from $5 \cdot 10^{5}$ effective quanta/burst. The decay of $\mathrm{J}$ or higher mass $\mathrm{J}-\mathrm{like}$ particles into multihadrons $(\pi, \mathrm{K}, \mathrm{p}$ detected) is expected to be $1(-3)$ orders of magnitude bigger.

For a resonance of $3.1 \mathrm{GeV}$ decaying into: $2 \mathrm{e} \quad 4 \mathrm{~K} \quad 6 \pi \quad 6 \mathrm{~K}$ the mass resolution is $\delta \mathrm{m}=110 \quad 50 \quad 38 \quad 7 \mathrm{MeV}$

The signature of reaction ( 3 ) is: a) obvious missing energy and momenta, b) $\mathrm{z}^{2}$ dependence of cross section. Better particle identification is another advantage for doing it at NAL rather than at Spear. 
EXPOSURE OF EMULSIONS TO 200-300 GeV $\pi^{-}$for NEW DETERMINATION OF MEAN LIFE OF $\pi^{\circ}$

P.S. Young (Mississippi State University)

Objectives: To determine the $\pi^{\circ}$ mean life by use of the 200-300 Gev $\pi$ interactions in nuclear emulsion.

Parameters to be measured: The quantities to be measured are the path lengths of $\pi^{0}$-decay through the Dalitz Mode. The precision in the measurement is primarily determined by statistical errors which depend on the number of Dalitz pairs analized. With 100 pairs we expect to obtain a total accuracy of better than 258 error.

Advantages of using $\pi^{-}$Beam: The production of $\pi^{\circ}$ from $\pi^{-}$makes use of the charge exchange reaction. Consequently we expect to obtain much longer path lengths than those in the pp collision as described in Experiment No. 195.

Exposure and Beam Time Requested: Three exposures are requested hereby for three emulsion stacks, consisting of 10 pellicles of $7.5 \mathrm{~cm} \times 18 \mathrm{~cm} \times 600 \mu$ each. The desired total intensities are $5 \times 10^{4}, 1 \times 10^{5}, 1 \times 10^{6}$ per $\mathrm{cm}^{2}$.

Remarks: This experiment is an extension of Experiment No. 195 for which the exposures of emulsion stacks to the $300 \mathrm{GeV}$ protons were carried out in last October. 


\section{EXPOSURE OF EMULSIONS TO $400 \mathrm{GeV}$ PROTONS FOR NEW DETERMINATION OF MEAN LIFE OF $\pi^{\circ}$ \\ P. S. Young (Mississippi State University)}

Objectives: To determine the $\pi^{\circ}$ mean life by use of the 400 GeV proton interactions in nuclear emulsion.

Parameters to be measured: The quantities to be measured are the path lengths of $\pi^{0}$-decay through the Dalitz Mode. The precision in the measurement is primarily determined by statistical errors which depend on the number of Dalitz pairs analyzed. We anticipate to obtain a total accuracy of better than $25 \%$ error with 100 pairs.

Advantages of using $400 \mathrm{GeV}$ Protons: The main difficulty in the determination of the $\pi^{\circ}$ mean life lies in the measurability of the path lengths which were of the order of several microns in the previous experiments. With the $400 \mathrm{GeV}$ protons beams, we have reason to expect to observe longer path lengths.

Exposure and Beam Time Requested: Three exposures are requested hereby for three emulsion stacks, consisting of 10 pellicles of $7.5 \mathrm{~cm} \times 18 \mathrm{~cm} \times 600 \mu$ each. The desired total intensities are $5 \times 10^{4}, 1 \times 10^{5}, 1 \times 10^{6}$ per $\mathrm{cm}^{2}$.

Remarks: This experiment is also an extension of Experiment No. 195 for which the exposures of emulsion stacks to the 300 GeV protons were carried out last October. 
A PROPOSAL TO STUDY MESON PRODUCTION AT I.ARGE $P_{\perp}$ WITH A $Y$-RAY DETECTOR

A. Barnes, K.W. Lai, J. Mellema, A.V. Tollestrup, R. L. Walker (Caltech); O. Dahl, R. Johnson, R. Kenney, M. Pripstein (LBL)

It is proposed to use the $\gamma$-ray detector and the 24 " long liquid hydrogen target now being used for Experiment \#lll to study production of $\pi^{\circ}, n^{\circ}$ and other mesons at large $P_{\perp}$ from $\pi^{ \pm} \mathrm{p}$ and $\mathrm{pp}$ interactions for $\mathrm{CM}$ angles from $30^{\circ}$ to $110^{\circ}$.

High transverse momentum $\left(P_{1}\right)$ phenomena have traditionally been associated with interactions at small distances. For the case of hadron-nucleon collisions they provide a tool to probe the structure of the nucleon, in particular to investigate its possible constituents. However, most of the observations obtained thus far are confined to the proton-nucleon interaction and very little data exist for meson-nucleon interactions. From the simple quark model of hadrons, only qq interactions were thus probed for producing large $P_{\perp}$ events. Whereas, in the case of meson-nucleon collisions one probes $\bar{q} q$ interactions also. There is a strong suggestion from the constituent picture of hadrons that inclusive meson production at high $P_{1}$ could be qualitatively different and considerably more copious for incident mesons than for incident baryons.

It is proposed to perform the experiment in two steps.

Phase I is to study pp $\rightarrow \gamma+x$ at $300 \mathrm{GeV} / \mathrm{c}$ in the $x-r e g i o n$ from 0 to 0.5 , using the same setup of Experiment \#11l in the $M 2$ beam without any essential change. There is good enough angular resolution to be able to identify the production of mesons decaying into two or more $\gamma$-rays, such as $\pi^{\circ}(2 \gamma), \eta^{\circ}(2 \gamma$ and $6 \gamma)$, $\omega^{\circ}(3 \gamma), f^{\circ}(4 \gamma)$, etc. The results of Phase I should help for the final planning of Phase II. A 100 hour run should be sufficient for this purpose.

Phase II is to measure large $P_{\perp}$ photons produced in $\pi^{ \pm} \mathrm{p}$ collisions at 100 and $200 \mathrm{GeV} / \mathrm{c}$, also in the M2 beam. Two positions of the $\gamma$-ray detector are anticipated, one around $\theta_{\mathrm{CM}}=$ $50^{\circ} \quad(x \simeq 0.5)$ and the other around $\theta_{\mathrm{CM}}=90^{\circ} \quad(x \simeq 0)$. To estimate a rate for observing $\pi^{ \pm p} C^{M} \pi^{\circ}+x$ at large $P_{1}$, they take $E \frac{\mathrm{d}^{3} \sigma}{\mathrm{d}^{3}}$ to be the same as for $\mathrm{pp} \rightarrow \pi^{\circ}+\mathrm{x}$ and independent of $\mathbf{x}_{11}$. With $2.5 \times 10^{12}$ protons/pulse on the Meson Area targer, they then obtain a rate of $\sim 1200 \pi^{\circ}$ events in the range $3<\mathrm{P}_{\perp}<4 \mathrm{GeV} / \mathrm{C}\left(-50 \pi^{\circ}\right.$ events above $\left.4 \mathrm{GeV} / \mathrm{c}\right)$ for a 200 hour run in either $\theta$ position with $200 \mathrm{GeV} / \mathrm{c} \pi^{-}$incident. An initial alf̧cation $8 \mathrm{f} 400$ hours is requested to study the dependence of $\mathrm{E} \frac{\mathrm{d} 3 \mathrm{\sigma}}{\mathrm{dp}^{3}}$ on incident momentum for pion-induced interactions and to make a comparison with proton-induced interactions. 


\section{MULTIPARTICLE PRODUCTION IN NUCLEI BY PROTONS OF SEVERAL HUNDRED GeV}

J. Cohen, E.M. Friedlander, M. Marcu, A.A. Martin, R. Nitu (Bucharest); A.J. Herz, O. Kofoed-Hansen (CERN); K. Gottfried (Cornell) Bucharest-CERN-Cornell Collaboration

It is proposed to study the multiplicity and angular distribution of particles produced in collisions of protons with heavy nuclei at 200 and $400 \mathrm{GeV}$. The targets will be very" thin wires of tungsten $(225 \mu \mathrm{m})$ and steel $(50 \mu \mathrm{m})$ which will be embedded in nuclear emulsion. The extremely high spatial resolution of the emulsion makes it relatively easy to observe and measure the fast particles in the projectile system, and the geometry of the experiment is such as to allow the simultaneous observation of the slow particles emitted in the process. We thus expect to be able to obtain detailed information on the fate of the projectile system as function of the atomic number of the target, and on the excitation of the target nucleus as determined from observation of the tracks of evaporated particles and knock-ons.

Note: The $400 \mathrm{GeV}$ part of the proposal is No. 292 .

Summary prepared by

$K$. Gottfried 
PROPOSAL TO MEASURE COHERENT DISSOCIATION OF $\pi, K$ AND $P$

INTO STRANGE PARTICIES AT NAL ENERGIES

P. Koehler (Fermilab); H. Courant, M. Marshak, E. Peterson, K. Ruddick (Univ. of Minnesota); T. Ferbel, Y. Fukushima, T. Jensen, B. Kern, F. Lobkowicz, W. Mollet, P. Slattery, E. Thorndike, D. Underwood (Univ. of Rochester).

Cross sections for the coherent excitation of particles impinging on nuclei rise with incident bombarding energy. Two processes are held responsible for these projectile excitations: (1) the interaction of the projectile with the nuclear coulomb field, and (2) the hadronic interaction between the colliding objects. The first process can be interpreted through an analysis paralleling the Primakoff effect, and can yield valuable information pertaining to radiative widths of resonances produced through coulomb dissociation. The second process, usually referred to as diffractive dissociation or Pomeranchukon exchange, is not currently understood in any fundamental way. The two separate types of interactions responsible for coherent dissociation are most readily distinguished through their differing dependences on nuclear mass, nuclear charge, scattering energy and momentum transfer. It is intended to examine both processes in sufficient detail so as to gain a significant advance in the understanding of the overall dissociation phenomenon.

Specifically, the objective of the experiment is the investigation, with high statistics, of the dependence of the cross sections on mass, momentum transfer, target and energy, for the two-body dissociation reactions:

$$
\pi^{-}+\pi^{-} \pi^{0}, \pi^{-} \rightarrow \mathrm{K}^{-} \mathrm{K}_{\mathrm{s}^{\circ}}^{0} \mathrm{~K}^{-}+\mathrm{K}^{-} \pi^{0}, \mathrm{~K}^{-} \rightarrow \mathrm{K}_{\mathrm{s}}^{0} \pi^{-}, \mathrm{K}^{-}+\Lambda_{\mathrm{p}}^{-}, \overline{\mathrm{p}} \rightarrow \bar{\Lambda}_{\mathrm{K}}^{-}
$$

The decay angular distributions and the states of $\Lambda(\bar{\Lambda})$ polarization will also be examined. This study will provide important new information concerning knowledge of radiative widths and of coherent processes, and will probe the detailed nature of the Pomeranchukon trajectory in the inelastic regime. It is proposed to execute this experiment utilizing a drift-chamber spectrometer system in conjunction with a high-precision $\pi^{\circ}$ detector. The experimenters request 900 hours of data taking in the M-l beam line to initiate these studies. 
PROPOSAL FOR A NEON EXPOSURE IN THE 30-INCH BUBBLE CHAMBER

G. Alexander, I. Bar-Nir, O. Benari, S. Dagen, J. Grunhaus,

L. G. Hyman, A. Levy, L. Lissauer, Y. Oren, L. Rosenstein (Tel-Aviv University)

It is proposed to study high energy proton-nucleon interactions (at several energies) in the $30 "$ bubble chamber when it is filled with neon. The principal objectives of this study are:

1) It is expected that the study of multiplicity, rapidity distributions, transverse momentum, correlations etc. when compared with the same quantities measured in $p-p$ collisions will yield valuable information on the role played by cluster formation in hadron-hadron interactions. There is particular interest in:

a. The mean number of clusters per interaction as a function of energy as well as their distribution.

b. The mean number of particles per cluster including neutrals.

c. The lifetime of clusters.

d. Information on the cluster-nucleon cross section.

2) Good $\gamma$-ray detection efficiency permits a high statistics study of the number of $\pi 0^{\prime} ' s$ as a function of the multiplicity, $\mathrm{f} O \mathrm{O}$ and higher moments. One can also study charged-charged, charged-neutral, neutral-neutral etc. correlations in a single experiment. It will be possible to search for $n^{\circ}$ and $\omega^{\circ}$ production.

3) It will be possible to study diffractive processes, with the neutral component visible. By comparing $n\left(\pi^{\circ}\right)$ with $\frac{n\left(\pi^{+}\right)+n(\pi)}{2}$ one verifies that only $\Delta I=0$ contributes.

4) Finally, the experiment will investigate the process $p p \rightarrow p p e^{+} e^{-}$as well as provide useful calibrations on total cross section and multiplicity for people working with cosmic rays.

Summary prepared by Y. Oren, February 1974 


\section{STUDY OF EVAPORATION AND SPALLATION EFFECTS IN PLASTIC VISUAL TRACK DETECTORS}

\section{R. Beaujean, W. Enge (University Kiel)}

Four stacks of plastic visual track detector sheets have been exposed to the $300 \mathrm{GeV}$ proton beam. The stack size was $5 \mathrm{~cm} \times 5 \mathrm{~cm} \times 3 \mathrm{~cm}$ the proton fluence about $10^{3}$ protons $/ \mathrm{cm}^{2}$ and $10^{5}$ protons $/ \mathrm{cm}^{2}$.

The intention of the experiment was to study the angular and energy distribution of secondary particles with charge $\mathrm{z}>2$ which are evaporation and spallation products originated from nuclear interactions of the protons with carbon and oxygen nuclei of the detector material. Simular processes induced by cosmic ray protons occur in our measurements on the isotopic composition of light cosmic ray nuclei. Since the detector is sensitive only to energy losses above a certain threshold the incoming proton is not detected. But some of the secondaries have the same registration range and therefore the same image as stopping light cosmic ray particles. The accelerator experiment was planned to find corrections on such secondaries in our cosmic ray measurements.

After irradiation part of the sheets were etched in $6 \mathrm{n}$ $\mathrm{NaOH}$ and scanned for tracks of heavy secondaries. Unfortunately the number of tracks was too small for a worthwhile analysis due to a too low fluence of primary protons. 


\section{A SEARCH FOR STABLE INTEGRALLY CHARGED, MASSIVE PARTICLES (HAN-NAMBU QUARKS)}

A. Van Ginneken (Fermilab), H. R. Heydegger (Purdue Univ. and Univ. of Chicago), C. M. Stevens (ANL).

A search for stable (or long lived), integrally charged $(+1$ or -1$)$, massive particles is proposed. These could be the fundamental particles of the type proposed by Han and Nambu. The detection method exploits the chemical properties of the atoms these particles would form when they are brought to rest. Positive particles would form hydrogen-like atoms, negative particles would attach themselves to ordinary nuclei and form an atom having one electron less than the original nucleus. Following chemical separations their identification and a rough measure of their mass would be obtained by mass spectroscopy.

On the basis of mass spectroscopic analysis, isotopic composition, chemistry and practical considerations suitable targets for the search of $(-1)$-charged particles appear to be $\mathrm{NaCO}_{3}$ and $\mathrm{CaO}$. These are to be placed, securely contained, in a beamdump. For (+1)-charged particles the target choice is less critical. However, the mass spectroscopic search would be more difficult since $\mathrm{H}, \mathrm{H}^{2}$ and $\mathrm{H}^{3}$, all copiously produced during the irradiation, are likely to interfere.

Assuming a sensitivity for the mass spectrometer of $10^{5}$ atoms/sample an upper limit of $10-35 \mathrm{~cm}^{2}$ for the production cross section of $(-1)$-charged particles could be established.

Between July and October of 1975 three vessels each containing about $1 / 3 \mathrm{~kg}$ CaO were present in the main dump of the dichromatic train of the Neutrino Lab. The dump received in excess of 1018 protons of $400 \mathrm{GeV}$. Chemical analysis of the targets is presently being performed.

Summary prepared by G. Giacomelli, March 1974 Updated. January 1976 A. Van Ginneken 
$\pi^{+}$AND $\mathrm{K}^{+}$INTERACTIONS AT $300 \mathrm{GeV}$ IN THE 30-INCH HBC

V.E. Barnes, D.D. Carmony, R.S. Christian, J.A. Gaidos, A.F. Garfinkel, L.J. Laszio, A.T. Laasanen, F.J. Loeffler, R.L. McIlwain, D.H. Miller, W. Morse, T.A. Mulera, T.R. Palfrey, K.L. Rangan, R.B. Willmann (Purdue University)

We plan to study $\pi^{+}$interactions on protons at an energy near $300 \mathrm{GeV}$ in the NAL 30-inch HBC using a tagged beam enriched by differential absorption of protons. In a 300,000 picture exposure at $270 \mathrm{GeV}$, for example, we estimate that there would be $26,000 \pi^{+} \mathrm{p}$ interactions, 7,300 $\mathrm{K}^{+} \mathrm{p}$ interactions, and $96,000 \mathrm{pp}$ interactions. We would measure all meson interactions and 108 of the pp interactions to give a direct comparison in the same system, which in addition to the bubble chamber would include the downstream proportional wire spectrometer plus whatever neutral particle detectors and particle identifiers may be available at the time. A second gas Cherenkov counter $\sim 350$ feet long will be used to separate $\mathrm{K}^{+}$from $\pi^{+}$, thereby turning an unacceptable $\mathrm{K}^{+}$ contamination into a useful (and very interesting) data sample.

The physics motivation is a general survey of the interactions of positive mesons at new energies, and includes the following:

1. Multiplicity distribution and its moments.

2. Single particle inclusive distributions, including neutrals, if the spark chamber spectrometer is used.

3. Particle correlations in multiparticle processes, including neutrals.

4. Diffractive dissociation of target and of meson beam: cross sections, mass distribution versus multiplicity, tests of factorization.

5. $\mathrm{v}^{\circ}$ production, hyperon production.

6. Resonance production, e.g. $\Delta$ using slow identifiable proton recoils.

7. Intercomparison of our $100 \mathrm{GeV} \pi^{+}, \mathrm{K}^{+}$data with the new $300 \mathrm{GeV} \pi^{+}, \mathrm{K}^{+}$data.

Summary revised by

V. E. Barnes, February 15, 1976 
A.S. Cary (Harvey Mudd College); J.E. Butt, D.T. King, C.R. Vane (University of Tennessee)

The proposal requests a nuclear emulsion experiment with $400 \mathrm{GeV}$ protons:

1. Beam: Protons $400 \mathrm{GeV}$

2. Intensity: $3 \times 10^{4} / \mathrm{cm}^{2}$

3. Emulsion: Two small stacks of Ilford K5, 600 thick, mounted on glass

4. Alignment: Parallel

These observations examine the proton-nucleus electromagnetic interaction cross section in a region where it is supposed to be rising with increasing energy. Previous experiments (NAL \#103, \#232) suggest that it may actually be declining.

Emulsion processing and scanning: Univ. of Tennessee

Received: January, 1974

Approved: March, 1974

Exposure: December, 1975. Beam intensity $\sim$ right.

Processed: Univ. of Tennessee

Comparison studies continues.

Additional data has been taken on $\pi^{-}$beam: $\pi A \rightarrow \pi \mathrm{Ae}^{+} e^{-}$at $200 \mathrm{GeV}(\# 264)$ in collaboration with P.S. Young.

Summary prepared by

D.T. King, January 1976 


\section{PROPOSAL TO STUDY P-D INTERACTIONS AT $205 \mathrm{GeV} / \mathrm{C}$ IN THE 30-INCH BUBBLE CHAMBER}

S. Barish, M. Derrick, T. Fields, L. Hyman, R. Miller,

B. Musgrave, J. Phelan, P. Schreiner, R. Singer and R. Smith

(Argonne National Laboratory); N. Fadeev, V. Grishin,

G. Jancso, T. Kanarek, E. Kladnitskaya, A. Kuznetsov,

N. Melnikova, M. Sabau, L. Shcheglova, M. Soloviev and

A. Solomin (Joint Institute for Nuclear Research, Dubna).

This is a proposal for the $30 "$ bubble chamber with the following requirements:

Bubble chamber

Hybrid system

filled with deuterium will make use of information from the downstream spark chambers, but do not consider the hybrid system essential for most of their objectives.

Beam

Tracks per picture

Number of pictures

Total No. of events

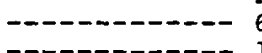

(1) $200 \mathrm{GeV} / \mathrm{c}$ protons 6 100,000

pn events with visible spectator- 11,000

The main emphasis of the proposal is the study of pn interactions, with an initial classification of events with seen and unseen spectator proton. The physics objectives are similar and complimentary to those of the pp exposure at $200 \mathrm{GeV} / \mathrm{c}$. In particular, they will study (a) charged particle multiplicity distributions; (b) diffraction dissociation, including $I=1$ exchange; (c) double charge exchange reactions; (d) inclusive $\mathrm{K}^{\circ}, \Lambda^{\circ}, \pi \pm$ production; (e) coherent events.

They should be able to have the first results 6 months after receiving the film; the analysis should be completed in two years.

Summary prepared by

G. Giacomelii, March 1974 


\section{STUDY OF HIGH ENERGY PI-MINUS \\ PROTON INTERACTIONS WITH THE NAL 30-INCH \\ BUBBLE CHAMBER-WIDE GAP SPARK CHAMBER HYBRID SYSTEM}

Iowa State Univ., Univ. of Maryland

Michigan State Univ., Univ. of Notre Dame

\section{G. A. Smith (Spokesman)}

This is an experiment of 300,000 pictures of $\pi^{-}$at $360 \mathrm{GeV} / \mathrm{c}$ in the 30" NAL bubble chamber with the wide gap spark chamber system of Experiment \#2B. The pictures were taken in August, September 1975 and scanning and measuring analysis is currently underway.

Besides the bubble chamber, the downstream experimental apparatus consists of four wide-gap spark chambers. The trigger is provided by five upstream beam deflection counters in conjunction with five downstream ones. The trigger requires that the incoming beam particle does not go through corresponding upstream and downstream beam deflecting counters, thus indicating that there was an elastic or inelastic event with $|\mathrm{t}| \approx 0.04(\mathrm{GeV} / \mathrm{c})^{2}$.

The data taken to date in Experiment \#2B have demonstrated that the hybrid system is a workable and a valuable instrument. It improves the momentum resolution of the forward going particles by more than a factor of ten over the bare bubble chamber measurement and provides information on $\pi^{\circ}$ 's produced in the entire forward hemisphere. study of:

The experiment will yield high statistics for a more significant

(i) correlations among charged particles;

(ii) exclusive channels;

(iii) inelastic diffractive and non-diffractive components;

(iv) individual topologies

Summary prepared by

G. A. Smith, January 1976 
SURVEY OF PARTICLE PRODUCTION IN PROTON COLLISIONS AT NAL

D. Carey, J. Johnson, R. Kammerud, D. Ritchie, J. Sauer,

R. Shafer, D. Theriot, J.K. Walker (NAL)

A $2.4 \mathrm{GeV} / \mathrm{c}$ double focusing magnetic spectrometer has been constructed to study the yields of single particles in the kinematic range $0 \leqslant|x| \leqslant 1$ and $0 \leqslant p_{\perp} \leqslant 2 \mathrm{GeV} / \mathrm{c}$ for proton-proton collisions up to $500 \mathrm{GeV}$. This experiment makes use of the forward-backward hemisphere symmetry in the proton-proton center of mass system. The reactions that will be studied are:

$$
\begin{array}{r}
p+p+p+\text { anything } \\
\bar{p}+\text { anything } \\
\pi^{ \pm}+\text {anything } \\
\mathrm{K}^{ \pm}+\text {anything } \\
\mu^{ \pm}+\text {anything } \\
\mathrm{e}^{ \pm}+\text {anything }
\end{array}
$$

The $s-$ and $t$-dependence of these single particle inclusive distributions will be studied over the ranges indicated. Approximately 150 hours of beam time are required at each beam energy that is available. It is hoped that measurements will be made from 50 to $500 \mathrm{GeV}$. In addition, 50 hours for beam tuning and 100 hours for equipment testing are requested. 
A SEARCH FOR A NEW STATE OF MATTER IN THE ANALYSIS OF A NAL BEAM DUMP

L.M. Lederman, I. Price (Columbia University); P. Limon (Fermilab)

This is a proposal designed to test a speculative implication of a theory due to T. D. Lee and G. C. Wick on the possible formation of an esoteric object with very high baryon number $(\mathrm{N}>400)$ which is stable and whose constituents are lower mass baryons. It could be possible that such a state be formed in a two-stage process initiated by high energy protons in a uranium beam dump.

$$
\begin{aligned}
& \mathrm{p}+\mathrm{U} \rightarrow \mathrm{U}^{\star}+\mathrm{p} \\
& \mathrm{U}^{*}+\mathrm{U} \rightarrow(\text { esoteric object })+\text { anything }
\end{aligned}
$$

The proposal requests:

1. To make available a high-z target already bombarded with $>10^{17}$ protons;

2. To install a uranium block surrounded by a lower-z material in an NAL beam dump.

3. To calibrate the exposure by a study of the probability of emission of heavy fragments from the bombardment of high $\mathrm{Z}$ nuclei with $300 \mathrm{GeV}$ protons using Lexan and Quartz detectors of very heavy ionizing particles.

The esoteric objects would be looked at by means of successive separations with diffusion processes, centrigues, and mass spectroscopy. 


\section{A STUDY OF DI-LEPTON PRODUCTION IN PROTON COLLISIONS AT NAL}

J.A. Appel, M.H. Bourquin, D.C. Hom, L.M. Lederman,

J.P. Repellin, H.D. Snyder, J. Weiss, J.K. Yoh (Columbia) ;

B.C. Brown, W. Innes, T. Yamanouchi (Fermilab)

It is proposed to study di-lepton production in protonnuclei collisions:

$$
\mathrm{p}+\text { nucleon } \rightarrow \mathrm{e}^{+}+\mathrm{e}^{-}+\text {anything }
$$

For this purpose the single arm electron spectrometer of Experiment \#70 will be transformed into a large solid angle, two-arm spectrometer, each with a hadron calorimeter and a muon filter. The equipment will be complemented with proportional wire chambers (about 5,000 wires).

The physics objectives may be summarized as follows:

1. Search for structure in the di-lepton spectrum.

2. The continuum of the effective di-lepton mass distribution is a measure of the spectrum of virtual photons emitted in the p-nucleon collisions. It may also be used for a test of parity violation due to interference of electromagnetic and weak interactions.

3. Qualitative study of the mass spectrum of hadron pairs $(\pi \pi, \pi p$, etc).

4. Check ue universality, studying $\mu^{+} \mu^{-}$pairs.

5. Extend the study of single lepton production of Experiment \#70.

6. Study of the $\pi^{\circ} \pi^{\circ}$ pairs via the conversion of the photons. 
SMALI ANGLE PROTON-HELIUM ELASTIC AND INELASTIC SCATTERING FROM 8 to $500 \mathrm{GeV}$

A. Kuznetsov, S. Mukhin, V. Nikitin, Y. Pilipenko (JINR, Dubna); E. Malamud, R. Yamada (Fermilab); S. Olsen, D. Nitz (Rochester); E. Jenkins (Arizona)

It is proposed to measure $\mathrm{p}-\mathrm{He}^{4}$ elastic scattering in the $0.1<|t|<0.3(\mathrm{GeV} / \mathrm{c})^{2}$ range and at energies varying continuously from 8 to $500 \mathrm{GeV}$. This proposal represents a logical continuation of work on $\mathrm{p}-\mathrm{p}$ and $\mathrm{p}-\mathrm{d}$ scattering.

The physics motivation may be summarized as follows:

1. Study of the energy dependence of $p$-He diffraction scattering, that is, of the slope for $|t|<0.2$ and of the minimum at $|t| \simeq 0.25(\mathrm{GeV} / \mathrm{c})^{2}$.

2. Computation of the part of the nucleon-nucleon scattering amplitude at $t=0$ as well as at $t \neq 0$ using Glauber theory. In particular, one is interested in knowing if the ratio of the real to the imaginary parts of the nucleon-nucleon scattering amplitude, $\rho(t \neq 0)$, goes through zero at an energy of 280 $\mathrm{GeV}$ as does $\rho(t=0)$.

3. Investigation of Glauber theory.

4. The scattering from a $J=0$ object, such as $\mathrm{He}^{4}$, at $t \neq 0$ may add useful information on spin effects at high energies.

5. Investigation of inelastic scattering, in particular, of the production of $\mathrm{N}^{*}(1400)$.

6. Determination of the jsobar-nucleon interaction cross section inside the $\mathrm{He}^{4}$ nucleus.

The experimental technique is similar to that used for $p-p$ (Experiment \#36A) and p-d (Experiment \#186) scattering using the c-0 internal target. The helium gas jet will require a special molecular sieve or condensed $\mathrm{CO}_{2}$ or Argon added to the tap cryosurface to contain the helium. Sandwiches of silicon (dE/dx, E) solid-state detectors will be used to separate $\alpha$-particles from background. The $0.1<|t|<0.3$ range corresponds to $40<\theta<70 \mathrm{mrad}$ from $90^{\circ}$, requiring better angular resolution than in the $p-p$ and $p-d$ scattering. This will be achieved by placing the detectors further away; quadrupoles may be added.

The experimenters request 200 hours of testing time and 500 hours for data taking, including some running at 400 and $500 \mathrm{GeV} / \mathrm{c}$. 
BACKWARD PION-PROTON ELASTIC SCATTERING

W.F. Baker, D.P. Eartly, A. Lennox, R. Rubinstein (Fermilab) and R. M. Kalbach, A. E. Pifer (Univ. of Arizona)

It is planned to measure the backward scattering of $\pi^{+}$ and $\pi^{-}$mesons on hydrogen at 10,50 and 80 (or 100 ) GeV/c in the angular range of $-0.8<u<0(\mathrm{GeV} / \mathrm{c})^{2}$.

They will study the energy and angular dependences of the elastic differential backward cross sections. Both will yield tests of Regge pole models in a relatively simple configuration where only two specific trajectories may be exchanged $\left(\Delta_{\delta}\right.$ in $\pi^{-} \mathrm{p} \rightarrow \mathrm{p}^{-}, \Delta_{\xi}$ and $\mathrm{N}_{\alpha}$ in $\left.\pi^{+} \mathrm{p} \rightarrow \mathrm{p} \pi^{+}\right) ;$the presently available high energy data disagree with the predictions.

The beam required should have $5 \times 10^{6}$ pions per burst. Such rates are possible in the $\mathrm{Ml}$ and $\mathrm{M} 6$ beam.

The incoming beam momentum will be defined to an accuracy of about \pm 0.28 by a scintillation counter hodoscope located at a momentum dispersed focus.

The experimental equipment consists of a liquid hydrogen target $1 \mathrm{~m}$ long, of a forward and of a backward spectrometer.

The forward spectrometer uses two NAL 24D72 (BMI09) analysis magnets and proportional wire chambers. The total length of the spectrometer will be increased with momentum according to the formula $\ell$ (feet) $=3 \mathrm{Po}(\mathrm{GeV} / \mathrm{c})$. The spectrometer will allow a momentum resolution $\Delta p / p= \pm 0.28$. Forward going pions will be placed in anticoincidence by a gas threshold cerenkov counter.

The backward spectrometer will use a 72018 magnet and proportional wire chambers. Its aperture is matched to that of the forward spectrometer and its momentum resolution is expected to be of the order of $\pm 4 \%$.

They will measure a complete angular distribution at one time. The rates anticipated from extrapolation of lower energy data are about 800 events/hour at $10 \mathrm{GeV} / \mathrm{c}, 50$ at $50 \mathrm{GeV} / \mathrm{c}$ and 5 at $100 \mathrm{GeV} / \mathrm{c}$. The highest momentum will be lowered or raised according to the rates experimentally found. The background is expected to be small at all energies and angles.

They request 200 hours of testing time and 900 hours of data taking. 
PROPOSAL TO STUDY MULTIPARTICLE PRODUCTION USING A NEON-HYDROGEN EXPOSURE

IN THE 15-FOOT BUBBLE CHAMBER

R. Ammar, R. Davis, C. Eklund, N. Kwak, L. Loos, R. Stump (University of Kansas); J. Whitmore (Michigan State University); J. Canter, F. T. Dao, W. A. Mann, J. Schneps (Tufts Untversity).

It is proposed to take two exposures of the FNAL 15-foot bubble chamber with a light neon-hydrogen fill ( 20\% mole neon) for a quantitative examination of the space-time structure of hadronic interactions at high energy. From a comparison of data for charged and neutral particle multiplicities, single particle inclusive spectra and multiparticle correlations, 8.3 observed in hadron-neon interactions, with analogous data from hydrogen bubble chamber exposures, the nature of multiparticle production in proton-nucieon collisions can be probed for small space-time intervals. Furthermore, the bydrogenic interactions are expected to provide unique information on neutral particle spectra and correlations by using the high probability for gamma-ray conversion and neutron interactions from individual events. The specific request is for 25,000 plctures with $400 \mathrm{GeV} / \mathrm{c}$ protons and 25,000 pictures with a $100 \mathrm{GeV} / \mathrm{c}$ Cerenkov-tagged secondary beam of protons and $\pi+1$ s. 


\section{MULTIPARTICLE PRODUCTION IN NUCLEI BY} PROTONS OF SEVERAL HUNDRED GEV

J. Cohen, E.M. Friedlander, M. Marcu, A.A. Marin, R. Nitu (Bucharest); A.J. Herz, O. Kofoed-Hansen (Cornell); K. Gottfried (Cornel1) Bucharest-CERN-Cornell collaboration

It is proposed to study the multiplicity and angular distribution of particles produced in collisions of protons with heavy nuclei at 200 and $400 \mathrm{GeV}$. The targets will be very thin wires of tungsten $(-25 \mu \mathrm{m})$ and steel $(50 \mu \mathrm{m})$ which will be embedded in nuclear emulsion. The extremely high spatial resolution of the emulsion makes it relatively easy to observe and measure the fast particles in the projectile system, and the geometry of the experiment is such as to allow the simultaneous observation of the slow particles emitted in the process. We thus expect to be able to obtain detailed information on the fate of the projectile system as function of the atomic number of the target, and on the excitation of the target nucleus as determined from observation of the tracks of evaporated particles and knock-ons.

Note: The $200 \mathrm{GeV}$ exposure has been designated No. 271. 


\section{A $\pi^{+}-\mathrm{d} 200 \mathrm{GeV} / \mathrm{c}$ BUBBLE CHAMBER EXPERIMENT AT FERMILAB}

Y. Eisenberg, B. Haber, D. Hochman, U. Karshon, E. Kogan, E.E. Ronat,

A. Shapira, R. Yarri and G. Yekutieli, (Weizmann Institute, Rehovot); and

H. Braun, D. Brick, F. Etienne, A. Fridman, E. Jegham, P. Juillot and

c. Voltolini (Centre de Recherches Nucleaires, Strasbourg)

Purpose: The main purposes of the experiment are:

a. Comparison of multiplicity distributions in $\pi^{+} \mathrm{p}, \pi^{+} \mathrm{n}$, pp and pn collisions.

b. Study and comparison of single particle spectra of slow charged $\left(\pi^{+}\right.$and $\left.\pi^{-}\right)$and neutral $\left(\pi^{\circ}, \Lambda^{\circ} / \Sigma^{\circ}\right.$ and $\left.k^{\circ}\right)$ particles in $\pi^{+} p$ and $\pi^{+} n$ collisions. The data will be compared with similar spectra in pp and pn collisions.

c. Study the fragmentation of neutrons into protons and measurement of the inclusive spectrum of protons in $\pi^{+} n$ and pn collisions for $-1<x<-0.3$. The inclusive reactions $\pi^{+} n \rightarrow\left(p \pi^{-}\right)+x$ and $\mathrm{pn} \rightarrow\left(\mathrm{p} \pi^{-}\right)+\mathrm{x}$ will also be studied in order to observe the possible production of the $\mathrm{N}^{*}(1470)$ isobar.

d. Study of the coherent reaction $\pi^{+} d \rightarrow$ (fast prongs) $+d$. In this reaction the incident proton is presumably fragmented by diffraction dissociation into fast particles with no isospin exchange. Study of the multiplicity distribution in this reaction can be used to test the various fits of high energy multiplicity distributions, based on the two component model.

e. Study of elastic coherent scattering $\pi^{+} d \rightarrow \pi^{+} d$, and measurement of do/dt for $|t| \geq 0.12 \mathrm{GeV}^{2}$.

Procedure:

a. Exposure: 150,000 pictures with $200 \mathrm{GeV}$ tagged beam of protons, $\pi^{+}, \mathrm{\mu}^{+}$and $\mathrm{k}^{+}$in the 30 in. bubble chamber filled with deuterium. The experiment will also use the downstream P.W.C. hybrid system. With 6 protons per picture 40,000 good $\pi^{+}$events are expected, namely about 500 events/mb.

b. Scanning: All types of events will be scanned for. The number of charged prongs, associated stopping tracks, vo decay and $\mathrm{e}^{+} \mathrm{e}^{-}$pairs will be recorded. The identify of the incident particle $\left(\mathrm{p}, \pi^{+}\right.$or $\left.\mathrm{K}^{+}\right)$ will be found from the data of the upstream tagging system.

c. Measurements: The momentum of all charged particles ( $<<15 \mathrm{GeV} / \mathrm{c}$ ) and associated $\mathrm{K}^{\circ}, \Lambda^{\circ}$ and $\mathrm{e}^{+} \mathrm{e}^{-}$pairs $(\mathrm{p}<20 \mathrm{GeV}$ ) will be measured. Protons and $\pi^{+}(p<1.4 \mathrm{GeV})$ will be identified by ionization measurements. The momentum of energetic charged particles in the forward direction will be found from the downstream P.W.C. data.

d. Coherent d-events: Deutrons having a range $\geq \mathrm{cm}$ can be identified in the bubble chamber by measurements performed on the slow particle, alone. This will correspond to coherent d-events with $|t|-0.16 \mathrm{GeV}^{2}$. By demanding coplanarity, coherent elastic d-events with $|t| \sim 0.12$ $\mathrm{GeV}^{2}$ could also be identified.

Progress: In september 1974, 53,000 pictures were taken in the 30" bubble chamber filled with deuterium and exposed to the tagged $200 \mathrm{GeV} / \mathrm{c}$ positive secondary had̆ron beam of proton, $\pi^{+}$at $\mathrm{K}^{+}$. 
QUARK SEARCH USING 400-500 GeV PROTONS (By Measuring Ionization Energy Loss)

L.B. Leipuner, R.C. Larsen, L.W. Smith, H.H. Williams (BNL); R. Kellogg, H. Kasha, R.K. Adair (Yale)

In June, 1973, data-taking for a quark search was completed in the M4 beam in the Meson Area. The experiment was done with the accelerator operating at $300 \mathrm{GeV}$, hence yielding a quark mass limit of approximately $12 \mathrm{GeV}$. More specifically, assuming a general production mechanism, they found that the production cross section for quarks of $1 / 3$ e or $2 / 3$ e was smaller than $10^{-35}$ $\mathrm{cm}^{2}$. A search was also made for particles of charge $4 / 3 \mathrm{e}$ achieving an upper limit on the cross section of $5 \times 10^{-31} \mathrm{~cm}^{2}$. It is planned to continue the search in the 30-inch hadron beam in the Neutrino Area and to push the mass limit higher (up to $\sim 15 \mathrm{GeV}$ for quarks of charge $1 / 3$ e and $2 / 3 \mathrm{e}$ ).

The basic equipment used in the search includes eight scintillation counters with outputs fed to two sets of discriminators. Their thresholds are set to a specific range of ionization corresponding to fractional charge. The recorded pulse heights further assist in the identification.

Approximately 24 hours of beam time is requested with the accelerator operating at $400 \mathrm{GeV}$ or above. Roughly half the time will be spent looking for $1 / 3$ e and $2 / 3$ e particles and half the time looking for those with charge $4 / 3 \mathrm{e}$. Through this run it will be possible to raise the mass limit and place an upper limit on the production cross section of approximately $10^{-35} \mathrm{~cm}^{2}$.

Summary prepared by

A.F. Greene, May 1974 
A PROPOSAL TO STUDY HADRON-NUCLEUS COLLISIONS AT FNAL ENERGIES USING 30" CHAMBER FILLED WITH PURE NEON

S. N. Ganguli, A. Gurtu, P. K. Malhotra, U. Mehtani, R. Raghavan and $A$. Subramanian (Tata Inst. of Fundamental Research)

\section{Sumnary:}

a. Bubble Chamber: 30" bubble chamber filled with pure neon and with $32 \mathrm{KG}$ field.

b. Beams: (i) Protons at $100 \mathrm{GeV} / \mathrm{c}$

(ii) Protons at $400 \mathrm{GeV} / \mathrm{c}$

(iii) Negative pions at $100 \mathrm{GeV} / \mathrm{c}$

c. Pictures: $10 \mathrm{~K}$ pictures (35 mm cameras - 3 views) for each of the three beams with about 3 tracks/picture

\section{Objectives:}

a. Hadron-neon absorption cross sections (to study multistep regeneration effects).

b. Multiplicity distributions of charged particles and $\gamma$-rays.

c. Effective cross-section of a system of hadrons against a nucleon for different multiplicities of the system.

d. Coherent inelastic production cross section.

e. Rapidity distribution of charged particles and $\gamma$-rays in the target fragmentation region. 
PRECISION STUDY OF HIGH ENERGY COLIISIONS INDUCED BY INCIDENT $150 \mathrm{GeV} / \mathrm{C}$ PIONS AND PROTONS

Brown University, Univ. of Cincinnati, Fermilab, IIT, Indiana University, University of Illinois, Johns Hopkins University, MIT, SUNY Albany, Oak Ridge, Rutgers University, Stevens Inst. of Technology, University of Tennessee, Yale University Irwin A. Pless (Spokesman)

The experiment will be performed using the Fermilab proportional Wire Hybrid $30^{\circ}$ Bubble Chamber System. They require 400,000 pictures each of $\pi^{+}, \pi^{-}$and protons with incident momentum of $150 \mathrm{GeV} / \mathrm{c}$. However, they have preliminary approval for only 600,000 pictures.

The objective of the experiment is the study of the following topics.

1. Charged particle multiplicity moments

2. Production spectra for charged and neutral particles

3. Leading particle and diffraction phenomena

4. Correlating in momentum and rapidity among the produced particles

5. Factorization of production amplitudes with respect to the quantum numbers of the incident particle.

In addition, we expect to make a preliminary study of $\mathrm{R}^{ \pm} \mathrm{p}$ and $\mathrm{\text {In }}$ interactions.

Summary prepared by

I.A. Pless, February 1975 


\section{STUDY OF PARTICLE PRODUCTION AT HIGH TRANSVERSE MOMENTA USING HYDROGEN AND DEUTERIUM TARGETS}

J.W. Cronin and H. Frisch (Univ. of Chicago); P.A. Piroué (Princeton University)

It is proposed to study the particle constituents of a beam produced at $80 \mathrm{mrad}$ lab angle $\left(\sim 90^{\circ}\right.$ in the p-p c.m. system) by 200-500 GeV protons striking a liquid hydrogen/ deuterium target. This work is basically an outgrowth of the investigation carried out in Experiment \#100A using targets of complex nuclei. The objectives of the proposed investigation with a hydrogen/deuterium target are:

1. To unfold the hadron production cross section for $p-p$ and $p-n$ collisions;

2. To make a more precise determination of the $\mathbf{s}$-dependence of the cross section than can be done with complex nuclear targets due to the effects of Fermi momentum;

3. To establish without ambiguity that no complex nuclear effects are affecting the data sample acquired in Experiment \#100A.

It is planned to complete the experiment in the P-East line of the Proton Area. The experimenters wish to make measurements for incident proton energies of $200,300,400$, and if possible $500 \mathrm{GeV}$. The estimated time required is 3 weeks for each energy. 
MEASUREMENTS OF HADRON-HADRON ELASTIC SCATTERING AT LARGE FOUR-MOMENTUM TRANSFER SQUARED

Cornell University, McGill University, FERMILAB, Northeastern University Marvin Gettner (Spokesman)

It is proposed to measure the hadron-proton $\left(\pi^{ \pm} p, K^{ \pm} p, \bar{p} p\right)$ elastic scattering differential cross sections at large angles to a sensitivity of $10^{-35} \mathrm{~cm}^{2} /(\mathrm{GeV} / \mathrm{c})^{2}$. As a first step it is planned to measure $\pi^{-}$p elastic scattering at 50 and $200 \mathrm{GeV} / \mathrm{c}$ in the $2<|t|<8(\mathrm{GeV} / \mathrm{c})^{2}$ range.

The measurement of hadron-hadron scattering at large $s$ and large $t$ provide information on strong interactions at small distances. Present high energy data includes mainly pp elastic scattering. The purpose of this experiment is to obtain data for the scattering of the other hadrons.

The proposed experimental method is the same as the one used for Experiment \#177A to measure pp elastic scattering. The equipment consists of a two-arm spectrometer system where the momenta and angles of scattered and recoil particles are measured. The forward spectrometer is made of two septum magnets which will yield a resolution of $\Delta t= \pm 0.5(\mathrm{GeV} / \mathrm{c})^{2}$. The large angle spectrometer is made of two BM-109 magnets, providing $\Delta \mathrm{p} / \mathrm{p} \simeq \pm 18, \Delta \theta / \theta= \pm 0.5 \mathrm{mr}$ and $\Delta \mathrm{t} \simeq \pm 0.1(\mathrm{GeV} / \mathrm{c})^{2}$.

The beam required is of $10^{8} \pi^{-}$per machine pulse. It is expected to have $\Delta \mathrm{p} / \mathrm{p} \simeq \pm \ldots .8, \Delta \theta_{\text {horiz. }}= \pm 0.5 \mathrm{mr}, \Delta \theta_{\text {vert. }}= \pm 0.3 \mathrm{mr}$ and a beam spot of \pm 0.1 " horizontal and \pm 0.2 " vertical. The incident beam will not be counted.

Because of the relatively large angular acceptance of the apparatus they expect to cover the $t$-range in two settings, one from 2 to $4(\mathrm{GeV} / \mathrm{c})^{2}$ and the other from 4 to $8(\mathrm{GeV} / \mathrm{C})^{2}$. The expected event rates should be of the order of 1 event per hour at $t=4$ with $\Delta t=0.1$ and 1 event $/ 20$ hours at $t=8$ with $\Delta t=1(\mathrm{GeV} / \mathrm{c})^{2}$. Backgrounds due to scattering from collimators and pole faces, inelastic collisions, randoms, and from neutrons should be small.

They request 200 hours for testing and 800 hours for data-taking for the $\pi^{-} p$ studies. 


\section{TEST OF C AT SMALL DISTANCES}

R. Cester-Regge, V. Fitch, M. Witherell (Princeton University); R. Turlay (Saclay)

It is proposed to measure a possible charge asymmetry in the momentum spectra of pions and kaons produced in pp inclusive reactions at $\theta_{\mathrm{c} . \mathrm{m} .}=90^{\circ}$ initiated by $200 \mathrm{GeV} \overline{\mathrm{p}}$ :

$$
\begin{array}{lrr}
\overline{\mathrm{p} p} \rightarrow \pi^{-}+\cdots & \overline{\mathrm{p} p} \rightarrow \pi^{+}+\cdots & \mathrm{R}_{\pi}=\pi^{+} / \pi^{-} \\
\rightarrow \mathrm{K}^{-}+\cdots & \rightarrow \mathrm{K}^{+}-\cdots & \mathrm{R}_{\mathrm{K}}=\mathrm{K}^{+} / \mathrm{K}^{-}
\end{array}
$$

Ten years after its discovery, the source of $C P$ violation in $K$ decay is still unknown. One may speculate that $C P$ violation could be a short distance phenomenon; in this view it is interesting to test other discrete symmetries at the shortest distances and at the highest momentum transfers.

The proposed experimental apparatus consists of two symmetric magnetic spectrometers, each viewing a $3 \mathrm{ft}$. long liquid hydrogen target at $97 \mathrm{mrad}$ in the laboratory, corresponding to $90^{\circ}$ in c.m. Each spectrometer has one 18D72 magnets, proportional wire chambers and two differential Cerenkov counters capable of separating $\pi$ from $K^{\prime} \mathrm{s}$ up to $50 \mathrm{GeV} / \mathrm{c}$. The range of transverse momenta which could be covered will be up to $5 \mathrm{GeV} / \mathrm{c}$. Great care will be exercised in reducing asymmetries. In principle the experiment could be done with a single arm spectrometer, but in all previous asymmetry measurements it was found high desirable to have two symmetric spectrometers.

The beam required is a $200 \mathrm{GeV}$ negative beam groduced by $400 \mathrm{GeV}$ protons. The intensity should be about $10 ? \pi$ and $4 \times 10^{5} \overline{\mathrm{p}}$ per $10^{12}$ protons on target. The incoming $\overline{\mathrm{p}}$ will be counted, while $\pi^{-}$and $k^{-}$will be anticoincidiced with Cerenkov counters.

In 400 hours of data taking with $1.6 \times 10^{5} \bar{p} /$ pulse they hope to achieve a charge asymmetry measurement of \pm 0.25 at $p_{t}=2$ $\mathrm{GeV} / \mathrm{c}$ for the production of pions and twice less accurate for kaons.

The apparatus is capable of yielding as by-products:

(i) The inclusive spectra at large transverse momenta (up to $\mathrm{p}_{\mathrm{t}}=4.5 \mathrm{GeV} / \mathrm{c}$ for $\mathrm{pp}$ collisions).

(ii) The mass spectrum of high mass objects (up to $\sim 10 \mathrm{GeV}$ ) which decay into two particles. 
PROPOSAL FOR A STUDY OF THE INTERACTION OF HIGH ENERGY $\pi \pm$ WITH URANIUM

W. D. Walker, L. Fortney, J. Loos, A. T. Goshaw, W. Robertson and J. Lamsa

(Duke University)

The proponents request $200 \mathrm{~K}$ pictures of the $30-$ inch Bubble Chamber filled with hydrogen and exposed to a $300 \mathrm{GeV} / \mathrm{c} \pi^{-}$ beam. Two thin heavy metal plates ( $\mathrm{Pb}$ or $\mathrm{U}$ ) would be placed in the chamber at the upstream end. Use of the downstream PWC Hybrid System and the new $\mathrm{Pb}$-Glass gamma-detecting spectrometer is requested.

The proponents state as their purpose for doing the experiment, the following six items:

1. To determine the multiplicity distribution produced in high energy $\pi$-Uranium interactions and to compare this data with data obtained in hydrogen and neon.

2 . To measure the inclusive momentum distributions of produced pions and protons and compare these data with the distributions found for neon and hydrogen.

3. To measure nuclear coherent $\pi A \rightarrow M A, M+3 \pi, 5 \pi$, etc.

4. To look at high $P_{\perp}(>1 \mathrm{GeV})$ events that are produced in the heavy nucleus.

5. To do a feasibility study for looking at $\pi-\gamma$ collisions. The $\gamma^{\prime}$ s being the virtual gammas produced by the high charge of a heavy nucleus.

6. To measure direct electron pair rates in a dense medium.

The proponents expect $\sim 5,000$ events in a $1-\mathrm{mm}$ plate of Uranium per $100 \mathrm{~K}$ pictures with four beam tracks per picture. They assume $1 \pi-\gamma$ interaction per every 20 events in the Uranium, thus an experiment of $200 \mathrm{~K}$ pictures should provide them with a data sample of 1000 such events. 
PROPOSAL TO STUDY THE COHERENT DISSOCIATION OF NEUTRONS

E. Bleser (FERMILAB); D. Freytag (Univ. of Massachusetts);

B. Gobbi, I. Kenah, R. Lipton, J. Rosen, R. Ruchti, H. Scott, D. Spelbring (Northwestern Univ.); J. Biel, C. Bromberg,

T. Ferbel, P. Slattery, D. Underwood (Rochester)

The participants in this proposal have recently completed an experiment (\#27A) to study diffraction dissociation from neutrons. This proposal is for an extension of that work with modifications to the equipment described below.

Experiment \#27A involved a study of the reaction $\mathrm{nz} \rightarrow \pi^{-} \mathrm{pz}$ over the large energy range of the neutron beam $(50-300 \mathrm{GeV})$ and with various nuclear targets from $B e$ to $U$. It is required that the interaction with the nucleus be coherent and the forward $V$ (from $\pi^{-p}$ ) is detected in a spectrometer system which includes an analysis magnet and wire spark chambers.

The preliminary data from Experiment \#27A shows the difference between the Coulomb dissociation (photon exchange) and diffraction dissociation (Pomeranchukon exchange) and the variation with target material (z). A study of the effective mass of the $\pi^{-} p$ system shows $\Delta^{\circ}(1236)$ production associated with Coulomb dissociation and $N^{*}(1470)$ production with diffractive dissociation. This study is presently limited to energies $2150 \mathrm{GeV}$ because of background. As part of this new experiment a neutron and a photon counter will be added to enable a study of the $n \rightarrow \pi^{+} \pi n$ and $n \rightarrow p \pi^{-} \pi^{\circ}$ diffractive processes and to eliminate them as background.

The physics interest in the new proposal includes:

1. Further study of the diffraction dissociation process including further examination of the $s$ - and $t$-dependence and mass dependence. Some of this will be done with a high pressure hydrogen gas target.

2. Study of dissociation of partially polarized neutrons involving changes in the $M 3$ beam.

3. Measurement of neutron and anti-neutron total cross sections from various targets including hydrogen and deuterium.

4. Search for two body decay of narrow mass resonances in the high mass region of neutron dissociation.

The experiment would utilize approximately five months of beam time interspersed with that for other experiments over the next year and a half. 
15-FOOT BUBBLE CHAMBER PROPOSAL FOR $485 \mathrm{GeV} / \mathrm{c} \pi^{-}$- p INTERACTIONS USING A TRACK SENSITIVE TARGET

A. R. Erwin, M. A. Thompson, E. H. Harvey and R. Loveless (University of wisconsin)

The proponents request $250 \mathrm{~K}$ pix of the 15-ft Bubble Chamber filled with a 338 atomic mixture of neon in hydrogen and exposed to a $\pi^{-}$beam of $485 \mathrm{GeV} / \mathrm{c}$. Use of a Track Sensitive Target and an upstream beam tagging system is assumed.

The proponents draw attention to four energy dependent phenomena which they would plan to study:

1. The average charged prong multiplicity as a function of the produced mass $M^{*}$.

2. The ratio of neutral particle to charged particle multiplicities.

3. The integrated correlation functions, $\mathrm{f}_{2}$.

4. The properties of events with $\pi^{\circ}$ 's produced at extremely large $\mathrm{p}_{\mathrm{T}^{*}}$

In each of these areas, the proponents emphasize the increased analysis power that will come as a result of the $\pi^{\circ}$ detection that will result in the $4 \pi$ geometry of the bubble chamber filled with the $\mathrm{NeH}_{2}$ mixture. In particular, they stress the importance of the correlations involving $2 \pi^{\circ} \mathrm{s}$. They believe it will also be possible for them to search for resonances which decay predominantly via a $\pi^{\circ}$ or electromagnetic decay mode.

The proponents believe the film can be analyzed using the SATR automatic measuring device at wisconsin. 


\section{FURTHER STUDY OF HIGH ENERGY NEUTRINO INTERACTIONS AT FERMILAB}

A. Benvenuti, D. Cline, A. Entenberg, W.T. Ford, R. Imlay T.Y. Ling, A.K. Mann, F. Messing, D.D. Reeder, C. Rubbia, R. Stefanski, L. Sulak, P. Wanderer and H.H. Williams Harvard-Pennsylvania-Wisconsin-FERMIIAB

This experiment has two specific physics goals, to study the matrix element for the muonless events and to study the deep inelastic scattering by charged currents at large $y$ (and $x$ ) over the neutrino energy range of 20-300 GeV.

These goals are accomplished by use of the present Exp. \#IA detector augmented with two new components, an energy flow device that measures the direction of the hadronic cascade that emerges from the muonless collision and a large angle magnetic muon spectrometer with large drift chambers. The complex of target-calorimeter-energy flow device is made of 8 units; each unit consists of a three-element system of liquid scintillator calorimeter cell, wide gap spark chambers and a Pb plate array that serves as a hodoscope and a $\gamma$-ray calorimeter simultaneously. An iron-plate calorimeter is placed at the end of the liquid scintillator-Pb calorimeter (at the beginning of the muon spectrometer) in order to completely contain the hadronic shower.

Other neutrino processes, such as those listed below, have a unique experimental signature in this detector and feasibility studies for measuring them will be carried out.

The experimenters plan to make full use of the versatility of neutrino beams which can be sent to their apparatus; they specifically mention the possibility of running with neutrino and antineutrino beams produced by 300 and $400 \mathrm{GeV}$ protons in the one-horn, two-horn, quadrupole triplet and dichromatic configurations.

Estimates are given below for the event rate per $10^{17}$ protons of $400 \mathrm{GeV}$ using the two-horn system (the second column is for the same reaction initiated by antineutrinos). $\nu_{\mu} \mathrm{N}+\mu^{-}+$hadrons $\nu_{\mu}^{\mu} \rightarrow v^{N}+$ hadrons $\nu_{\nu}^{\mu} e_{\mathrm{Pb} \rightarrow \nu}^{-} \mu_{\mu} e^{-}{ }_{\mu}{ }^{-} \mathrm{Pb}$ $\nu_{\mu}^{\mu} \nu_{\mathrm{N} \rightarrow \mathrm{e}^{-\mu}}+$ hadrons 46,000 events 4.600 10,000 events $\nu_{e}^{e} e^{-}+\nu_{e} e^{-}$

1.8

3.100

1.2

3.5

460

23

For the first reaction it should be possible to investigate the $\left(Q^{2}, E_{H}\right)$ region, $Q^{2}<200(\mathrm{GeV} / \mathrm{C})^{2}$ and $E_{H}<200 \mathrm{GeV}$.

Use of a large hydrogen target is also envisioned as a possible future addition. 
PROPOSAL TO STUDY MULTIPARTICLE PRODUCTION IN HIGH ENERGY ANTIPROTON-PROTON INTERACTIONS WITH THE NAL 30-INCH BUBBLE CHAMBER

R.E. Ansorge, J.R. Carter, J.G. Rushbrooke and B.R. Webber (Cavendish Laboratory); W.W. Neale, L. Voyvodic, R.J. Walker and $J$. Whitmore (Fermilab); Z. Ming Ma, W. Morris, B.Y. Oh, D.L. Parker and G.A. Smith (Michigan State)

We propose to study multiparticle production in the interactions of $100 \mathrm{GeV} / \mathrm{c}$ antiprotons in the 30 -inch hydrogen bubble cahmber. We request 100,000 pictures with a Cerenkov tagged. secondary beam containing roughly equal numbers of $\bar{p}$ 's and $\pi^{-}$'s. We also plan to use the downstream wide gap spark chamber hybrid system with a few minor modifications to study neutral particle producton in the forward direction.

Summary prepared by

w. W. Neale, May 1974 


\section{HADRONIC INTERACTIONS IN COMPLEX NUCLEI}

R.M. Edelstein, D.R. Green, H.J. Halpern, J.S. Russ (Carnegie-Mellon University)

It is proposed to study several types of hadronic interactions in complex nuclei using the Multiparticle Spectrometer Facility (MPS) in M6 (West) at FERMILAB. Broadly speaking, these experiments are for the purpose of learning about the following:

1. Diffraction dissociation, through the use of the unique filter of coherent hadron dissociation.

2. The interaction of high energy hadronic systems in nuclear matter, including production, absorption, and cascade.

3. Manifestly incoherent beam dissociation reactions such as those with charge exchange.

4. Details of nuclear models for coherent and incoherent scattering.

The MPS facility is a magnetic spectrometer with proportional wire chambers, wire spark chambers and Cerenkov counters for particle detection. It will be used first to perform approved Experiments \#110A and \#260. This experiment is proposed for a time when it is expected that the spectrometer as well as beam detection equipment will be functioning. Most revisions of the apparatus are required in the form of $\mathrm{Pb}-$ scintillator sandwich counters 1) downstream of the spectrometer for $\pi^{\circ}$ detection, and 2) surrounding the target for $\pi^{\circ}$ and nuclear breakup detection.

The running time requested is 200 hours for testing and 800 hours for data taking. A minimum of 6 calendar months is needed for setting up, testing and running. 
POLARIZATION IN P-P ELASTIC, INELASTIC AND INCLUSIVE REACTIONS AT NAI ENERGIES

G.W. Abshire, B.B. Brabson, R.R. Crittenden, A. Dzierba, S. Gray, R.M. Heinz, J.E. Mott, H.A. Neal, D.R. Rust (Indiana University)

It is proposed to utilize the expanded Internal Target Area and the hydrogen jet target and recoil spectrometer facility to measure the polarization in $p-p$ elastic, quasi-elastic, and inclusive scattering. The motivation for such a study originates with the wish to determine the energy dependence of the fixed-t polarization over an energy range corresponding to beam energies from 20 to $400 \mathrm{GeV}$.

In Regge models the recoil proton polarization in $p-p$ elastic scattering is due to the interference of participating Regge trajectories and the prediction for the variation of the fixed-t polarization at large $s$ is particularly simple. A significant deviation of the fixed-t polarization from a power law in s would be of substantial interest. It will also be of interest to determine if the double zero in the polarization $t$ distribution observed near $|t|=.7(\mathrm{GeV} / \mathrm{c})^{2}$ at all momenta below $20 \mathrm{GeV} / \mathrm{c}$ remains as a systematic feature of the protonproton system at very high energies. This feature has been accounted for in certain optical and parton models.

The new item of equipment for the experiment is a carbon polarimeter capable of measuring the recoil polarization to an accuracy of $\sim 18$. The analyzing efficiency for this device will be greater than .005 at all proton energies covered by the experiment. The polarimeter consists of a block of carbon (of variable thickness) preceded and followed by groups of proportional chambers.

Estimates of running time are for 800 hours to collect data on $p-p$ elastic scattering at -6 t-values from .2 to 1.0 $(\mathrm{GeV} / \mathrm{C})^{2}$ and 5 s-values from 63 to $752(\mathrm{GeV})^{2}$. The polarization in $\mathrm{N}^{*}$ and inclusive production would require an additional 200 hours for data-taking to cover t-values below $1(\mathrm{GeV} / \mathrm{c})^{2}$. The inclusive studies are expected to lead to a proposal to pursue such work in detail at a later time. 


\section{PROTON DIFFRACTION DISSOCIATION ON HYDROGEN AND DEUTERIUM}

JINR (Dubna), FERMILAB, Rockefeller Univ., Univ. of Rochester S. Mukhin (Spokesman)

It is proposed to extend the study of inelastic scattering begun in Exp. \#186 and to compare the behavior of the inelastic cross sections for $p d+X d$ to that for $p p+X p$ over the same mass and $t$ range. The earlier work of Exp. \#186 was done with a deuterium gas jet and involved a study of coherent scattering of protons from deuterons. In subsequent analysis the inelastic proton-nucleon cross sections have been unfolded using the devteron form factor. Those results show a $1 / \mathrm{M}_{\mathbf{X}}^{2}$ dependence of $\mathrm{d}^{2} \sigma / \mathrm{dtdM}_{\mathrm{x}}^{2}$ as is predicted by the triple-Regge formula with pure Pomeron only. Also the $t$-dependence of the cross section for a specific range of $\mathrm{M}_{\mathrm{x}}^{2}$ is linear, suggesting a discrepancy with the earlier results studying inelastic reactions from protons (Exp. \#14A) which showed a turnover in do/dt for $|t|<0.1(\mathrm{GeV} / \mathrm{c})^{2}$.

It is planned to continue the study of these inelastic reactions using hydrogen, where the $t$-dependence of the deuteron form factor would not have to be considered, and comparing these results with coherent deuterium measurements. Some additional solid state detectors would be added to the existing system enabling a $t$-coverage of $0.01<|t|<0.1 \quad(\mathrm{GeV} / \mathrm{c})^{2}$ and an $\mathrm{M}_{\mathrm{X}}{ }^{2}$ out to $60 \mathrm{GeV}^{2}$ at a beam momentum of $300 \mathrm{GeV}$.

The running time request is for approximately two months of data-taking with the jet operating at 4-6 shifts per week.

Summary prepared by

A.F. Greene, June 1974 


\section{PION DIFFRACTION DISSOCIATION}

G. Ascoli, R. Klanner, L.J. Koester Jr., V.E. Kruse, R.D. Sard (Univ. of Illinois), D. Garelick, M. Glaubman, E. von Goeler, D. Potter, H. Johnstad (Northeastern Univ.)

The broad aim of this proposal is to study pion diffraction dissociation at the highest available energies. Diffraction dissociation is here meant as $\pi \pm \mathrm{p} \rightarrow \mathrm{pX} \pm$ (with $\mathrm{x}^{ \pm}$observed in detail) at modest values of $p_{t}$ and of $M^{2} x$. Specific purposes will be to confirm the diffractive nature of $\mathrm{A}_{2}$ production as observed at Serpukhov energies and to test particle production models like the triple Regge formula.

They plan to use the Chicago Cyclotron spectrometer in the present configuration used by Experiment \#98, with the following modifications: (i) use of $30 \mathrm{~cm}$ liquid hydrogen target, (ii) addition of a proton recoil detector consisting of one proportional wire chamber, three magnetostrictive wire chambers and two scintillation counter hodoscopes, and (iii) ignoring the last part of the equipment, after the $\gamma$-ray detector.

The setup will be triggered in different ways: (i) "bubble chamber mode" in which the spectrometer is triggered by any inelastic interaction in the hydrogen target. They expect 750,000 of these events. They will be used to study single particle spectra, correlations in inclusive distributions, and general features of the interactions. (ii) proton trigger events. In the reactions $\pi{ }^{ \pm} p \rightarrow p x \pm$ the proton has to be detected in the proton recoil detector. This limits the kinematically accepted ranges to $0.05<|\mathrm{t}|<0.45(\mathrm{GeV} / \mathrm{c})^{2}$ and $\mathrm{M}_{\mathrm{X}}<4 \mathrm{GeV}$. They propose to take 2.5 million triggers. Particular care will be reserved to the fully measured reactions The acceptance of the spectrometer, though not as good as in a bubble chamber, covers most of the forward hemisphere, and is better than for previous spectrometers. The presence of a photon detector will help in separating the type of reaction. (iii) coherent production of $3 \pi, 5 \pi$ systems from nuclei $(\pi \mathrm{Z} \rightarrow(3 \pi) \mathrm{z})$. For this purpose the hydrogen target and proton recoil detector will be replaced by a heavy $\mathrm{z}$ target surrounded by anticoincidence counters designed to remove all events with particles produced at large angles and with $|t|>0.2$ $(\mathrm{GeV} / \mathrm{c})^{2}$.

They plan to run with negative pions at momenta of 50,100 and $150 \mathrm{GeV} / \mathrm{C}$ and at one momentum for positive pions. Beams of $\lesssim 10^{5}$ pions per burst are adequate. They estimate a need for about 300 hours of testing and 1000 hours of data-taking. 
FURTHER TEST OF SCALING AT HIGH MOMENTUM TRANSFERS IN DEEP INEIJASTIC MUON SCATTERING

C. Chang, K.W. Chen, A. Kotlewski, I. Litt, R. Renninger, and P. Schewe (Michigan State University)

This proposal is a follow up of Experiment \#26. The main purpose is to test scale invariance in muon-nucleus deep inelastic scattering at the highest $\mathrm{q}^{2}$. During the past three years the Exp. \#26 group originated, developed, and operated a magnetized iron spectrometer in the Muon Laboratory. The preliminary results of the experiment indicate a possible deviation from scaling at large $q^{2}$.

It is proposed to increase the $\mathrm{q}^{2}$ and $v$ ranges covered by the experiment by: (i) placing the targets and magnets closer together, (ii) running at higher muon energy and higher intensities (for this purpose proportional wire chambers will be placed upstream and downstream of the target where the rates are higher), (iii) changing the target to 20 feet of heavy $z$ target which will be segmented with scintillation counters every 4" to aid in vertex identification. Using a muon beam of $240 \mathrm{GeV}$, they expect to be able to cover the range $0<q^{2}<160$ $(\mathrm{GeV} / \mathrm{C})^{2}$ and $40<v<210 \mathrm{GeV}$.

Three muon beam energies are contemplated for the test of scaling: 90,150 and $240 \mathrm{GeV}$. The original method of Exp. $\$ 26$, to geometrically scale the apparatus, will be used again. Both $\mu^{+}$and $\mu^{-}$are desired so as to measure the ratio of $\mu^{+}$to $\mu^{-}$deep inelastic cross sections. It is also planned to use $\mathrm{Be}, \mathrm{Fe}, \mathrm{Pb}$ and $\mathrm{W}$ targets.

The data-taking will require $5 \times 10^{11}$ muons corresponding approximately to 1100 hours of running.

Sumnary prepared by

G. Giacomelli, June 1974 
PROPOSAL TO MEASURE NEUTRAL CURREIJT CROSS-SECTIONS AIND ASSOCIATED INELASTIC DISTRIBUTIONS IN THE NARROW-BAND BEAM

B.C. Barish, K. Brown, F. Bartlett, D. Buchholz, F. Jacquet, F. Merritt, F. Sciulli, L. Stutte, H. Suter (Caltech), G. Fisk, G. Krafczyk (FERMILAB)

They propose to continue the neutral current investigation begun in Exp. \#262 with measurements of the absolute cross section and of the $y$-distribution of neutral current events.

In the Weinberg model the $y$-distribution of neutral current events is expected to have the following form

$$
\frac{d \sigma^{v}}{d y} \simeq N\left[r+s(1-y)^{2}\right] \quad \frac{d \sigma^{v}}{d y} \simeq N\left[r(1-y)^{2}+s\right]
$$

where is depends on the neutral current coupling and on nuclear structure functions; $r$ and $s$ depend on the weinberg angle. Thus both the $y$-distribution and the overall normalization are of considerable theoretical importance.

Experimentally it is difficult to measure events with low hadron energies and thus at low $y=E_{h} / E_{v}$. In order to minimize experimental biases it is preferable to use high energy neutrinos. The setup of the dicromatic neutrino beam allows a cha ge of the relative number of $\pi$ - and $k$-neutrinos, by steering the hadron beam relative to the neutrino detector (the kaon neutrinos are enhanced by a factor of 10 relative to the pion neutrinos if the angle is changed from $0 \mathrm{mrad}$ to 1.75 mrad).

Using the same equipment of Exp. \#21A (and \#262) and an enhanced (monochromatic) kaon neutrino beam of high energy they estimate they are capable of measuring the absolute angular distribution down to $y=0.06$. Based on the rates from Exp. \#262 they request $3 \times 1018$ protons with a fast spill on the neutrino target to measure the absolute neutral current and inelasticity distributions for both neutrinos and anti-neutrinos with different neutrino beam conditions. They could start running in September 1974 . 
A HIGH PRECISION EXPERIMENT TO MEASURE THE INELASTIC $p-p$ CROSS SECTION AND ITS ASSOCIATED FORWARD MULTIPLICITIES AT SMALI MOMENTUM TRANSFER

S. Childress, P. Franzini (Columbia Univ.); F. Bletzacker, J. Lee-Franzini, R. McCarthy, R.D. Schamberger (SUNY, Stony Brook)

This group has completed an experiment to study inelastic proton-proton collisions (Exp. \#14A) at 200, 300, and $400 \mathrm{GeV}$ using an external proton beam in the Neutrino Area. Exp. \#221, now in progress, is a further study of these processes using the internal proton beam at the Internal Target Area. The group is proposing yet another extension of this work, still at the Internal Target Area but now using a hydrogen gas jet instead of relying on carbon-polyethylene subtraction. They also plan to study the associated forward multiplicity of the inelastic collisions.

Preliminary results from Exp. \#221 show fits to the inelastic cross section, $\mathrm{d}^{2} \sigma / \mathrm{dtdM}_{\mathrm{x}}{ }^{2}$, to require a constant term plus a $1 / M_{X}^{3}$ term; the former is energy dependent and the latter is not. This compares with results from other experiments covering a similar kinematic range which suggest an energy independent $1 / M_{x}{ }^{2}$ behavior consistent with pure pomeron exchange in a tripleRegge formula. Results from Exp. \#14A also show a non-exponen$t i a l$-dependence below $|t| \approx 0.1$.

The experiment progosed will cover the regions: $0.011<|\mathrm{t}|<0.234(\mathrm{GeV} / \mathrm{c})^{2}, 0.75<\mathrm{x}<1.0$ and $\mathrm{Mx}^{2}$ up to $200 \mathrm{GeV}^{2}$ at $480 \mathrm{GeV}$ beam momentum. Measured resolution is expected to be: $\Delta M_{x}{ }^{2}=0.5 \mathrm{GeV}^{2}, \Delta t / t \simeq 2 \%, \Delta s / s \simeq 2.5 \%$. The following changes in equipment are proposed: i) a new low intensity hydrogen jet $\left(10^{3}-10^{4}\right.$ times smaller than the present jet target), ii) an extended ion chamber with radiation protection to be provided by the expansion of the Internal Target Area, and iii) a downstream scintillator hodoscope for multiplicity measurements.

The experimenters ask for 3-6 months for testing and 6-9 months for data-taking assuming beam time availability of $30 \%$.

Summary proposed by

A.F. Greene, June 1974 


\section{SINGLE-PARTICLE SPECTRA IN HIGH-ENERGY COLLISIONS OF CHARGED HADRONS}

E. Beier, H. Brody, G. Featherston, K. Raychaudhuri, H. Takeda, R. Thern, R. Van Berg and H. Weisberg (University of Pennsylvania)

This experiment is proposed to measure the differential cross section for the inclusive reactions $a+b \rightarrow c+$ anything, where $a, c=p, \bar{p}, \pi^{ \pm}$and $k^{ \pm}$, and $b$ is a target proton. Threshold Cerenkov counters in the incident beam and in the scattered particle spectrometer are used for particle identification. In each phase incident beam momenta for 40,80 , and $160 \mathrm{GeV} / \mathrm{c}$ will be used.

The beam requirements are for maximum available intensity that the Cerenkov counter can handle (for most of the run), with a $1 \mathrm{~cm} \times 1 \mathrm{~cm}$ spot size and angular divergence better than $\pm 2.5 \mathrm{mrad}$. It is proposed to place the equipment in the "front porch" area of the $3.5 \mathrm{mrad}$ beam (M1).

The spectrometer is now being used at the AGS in Experiment 555 (Single Farticle spectra). It pivots mechanically around the target and uses proportional chambers. A small C-magnet (12C24), an on-line computer link, a $\mathrm{LH}_{2}$ target and certain beam-line instrumentation are required from Fermilab.

The experiment covers secondary moments $p_{1 a b} \leqslant 20 \mathrm{GeV} / \mathrm{c}$ corresponding to the "target fragmentation" and "central" region of particle production, $-1.0 \leqslant x \leqslant 0.1$.

The total estimated time for running is 800 hours. 


\section{STUDY OF DI-MUON PRODUCTION EMPLOYING A PROTON BEAM STRIKING A COPPER TARGET}

J. P. Boymond, P. A. Piroue, R. L. Sumner (Princeton University); J. W. Cronin, H. J. Frisch, M. J. Shochet (University of Chicago)

As part of the recent interest in direct production of muons in high energy interactions including their possible origination from decays of vector mesons, one of the groups which originally observed these phenomena (in Exp. \#100A) is proposing a further examination of these reactions in subsequent work with the same apparatus. Experiment \#300 is intended as an extension of the particle production work from heavy nuclear targets but now using hydrogen and deuterium targets. As part of that running a copper target would be used to look for di-muon production. A second arm to detect direct muons coincident with a count in the main spectrometer will be constructed. This arm will consist of $1012^{\prime}$-high by $4^{\prime}$-wide liquid scintillation detectors placed in an array of $4^{\prime}$ diameter $\times 20^{\prime}$ deep holes. The detectors are placed on a line displaced $15^{\prime}$ from the west edge of the Proton-East pit. Muons produced at the target are required to have $p_{+} \approx 2 \mathrm{GeV}$ to penetrate the earth between the target and the detectors. In the $\mathrm{cm}$ system this detector system (Multi-Hole Spectrometer) will subtend a polar angle of between $45^{\circ}$ and $120^{\circ}$ and an azimuthal angle of $33^{\circ}$. A movable absorber placed near the target will enrich the direct muons arriving at the detector.

Using a parton-antiparton annihilation model it is estimated that about $2 \%$ of the events with a direct muon in the primary spectrometer with $p_{L} \simeq 3 \mathrm{GeV} / \mathrm{c}$ would have a muon coincidence in the second arm. Chance coincidences in the muon detector are estimated to be $3 \times 10^{-3}$. The effective mass of the muon pair will be calculated from the angle and momentum of the muon in the spectrometer arm and from the angle of the second muon measured with the other arm. The mass resolution, $\delta M / M$, is estimated to be about $10 \%$. The mass range covered is $3<M_{\mu \mu}<15 \mathrm{GeV} / \mathrm{c}^{2}$ with a cross section sensitivity $\sim 10^{-39} \mathrm{~cm}^{2}$. 
A PROPOSAL TO MEASURE MUON PAIRS PRODUCED AT HIGH TRANSVERSE MOMENTUM BY PIONS

J.W. Cronin, H.J. Frisch, M.J. Shochet (Univ. of Chicago); J.P. Boymond, P.A. Piroué, R.L. Sumner (Princeton)

It is proposed to measure the direct di-muon production by $200 \mathrm{GeV}$ pions

$$
\pi^{-} \mathrm{p} \rightarrow \mu^{+}+\mu^{-}+\text {anything. }
$$

Experiments \#70, \#100A and \#184 have recently observed direct muon production at large $p_{t}$ in nucleonnuclei collisions. The yields are $210^{-4}$ of the pion yield, essentially independent of $E_{O}, p_{t}$ and $\theta_{\mathrm{cm}}$. These muons could come from the decays of vector mesons or from new phenomena. It is thus important to study the direct production of di-muons, in particular in pion initiated reactions. The parton model predicts that di-muon production by pions should be considerably larger than in nucleon-nucleon collisions, because the pion has a fast antiquark content, which facilitates the quarkantiquark annihilation into a high mass virtual photon which then decays into a muon pair.

They will use the high intensity pion beam and the focusing spectrometer of their approved Experiment \#258, to which they will add a second arm, made of scintillation counter hodoscopes placed after some shielding. The focusing spectrometer will be at an angle of $100 \mathrm{mr}$, while the added muon counters will cover the angular range between 35 and $160 \mathrm{mr}$ in the lab. This experiment will be intrinsically parasitic on Experiment $\$ 258$. They anticipate a counting rate of 8 di-muon events per shift when the incoming pion momentum is $200 \mathrm{GeV} / \mathrm{c}$ and the focusing spectrometer is set at $\mathrm{p}_{t}=3 \mathrm{GeV} / \mathrm{c}$ (invariant mass of $6 \mathrm{GeV}$ ). 
TESTS OF PARTICLE IDENTIFICATION BY IONIZATION LOSS (ISIS)

W.W.M. Allison, C.B. Brooks, N.N. Bunch, P. Shield (Oxford); R. Yamamoto (MIT)

The mnemonic ISIS is to describe "identification of secondaries by ionization sampling." The oxford group has been experimenting with a large multi-wire drift chamber to distinguish between pions, kaons, and protons with momenta from $5 \mathrm{GeV} / \mathrm{c}$ to about $100 \mathrm{GeV} / \mathrm{c}$. Cerenkov counters can furnish only a limited number of cells and consequently have difficulty in separating multiple secondary particles, and transition radiation detectors are believed to be useful only in the region of several hunared $\mathrm{GeV} / \mathrm{c}$.

The ISIS effort has been directed toward using the relativistic rise of ionization loss in gases at atmospheric pressure. This rise is due to the effect of the relativistic expansion of the transverse electric field of a particle.

One difficulty is Landau fluctuations which tend to mask the correlation between particle energy and ionization. However, it is believed that with the selection of a proper gas and by measuring many samples on each track that this technique could succeed.

These data will also allow detailed quantitative comparison with theoretical calculations of loss distributions at different velocities. Significant differences between available data and earlier calculations have been understood and better agreement achieved by Monte Carlo calculation based on the single scattering collision probability as described by U. Faro. These calculations give absolute predictions for the results of this experiment.

The experimenters propose to test a prototype ISIS chamber in the hadron beam of the Neutrino Area at Fermilab. It would be possible to compare their results with a conventional tagging Cerenkov counter also in that beam. There is a need for approximately one shift of data-taking at the positive beam momenta of $25,50,100$ and $175 \mathrm{GeV} / \mathrm{c}$, one negative momentum and some miscellaneous checks.

Summary prepared by

W. Allison, January 1975

Addendum, January 1976: The analysis of the data is complete and the final results have been written up (oxford University preprint $67 / 75$ and $68 / 75$ ) and are to be published (Nuclear Instruments and Methods). The results indicate that the Monte Carlo model calculations are correct and that particles may be identified using this technique. 


\section{THE INTERACTIONS OF $\pi^{-}$-MESONS IN NUCLEAR EMULSION AT $200 \mathrm{GeV} / \mathrm{C}$}

M.I. Adamovich, N.A. Dobrotin, M.I. Tretjakova (P.N. Lebedev Physical Institute of the Academy of Science, USSR)

The principal interest in this experiment is to investigate the characteristics of $\pi^{-}$-nucleon and $\pi^{-}$-nucleus coherent and incoherent interactions at $200 \mathrm{GeV} / \mathrm{c}$. Comparison will be made with observations previously by this group using lower energy pions, at 17 and $60 \mathrm{GeV}$, as well as with $200 \mathrm{GeV}$ proton exposure.

Summary prepared by

L. Voyvodic, May 1976 
THE INTERACTIONS OF PROTONS IN NUCLEAR EMULSION AT $400 \mathrm{GeV} / \mathrm{C}$

M.I. Adamovich, N.A. Dobrotin, M.I. Tretjakova (P.N. Lebedev Physical Institute of the Academy of Science, USSR)

The purpose of this experiment is to continue the study of interactions in nuclear emulsions by high energy protons. An exposure with $400 \mathrm{GeV}$ protons in this experiment will allow systematic studies, and comparison with previous studies at 67 and $200 \mathrm{GeV}$, on multiplicities and angular distributions of secondary particles for both proton-nucleon and protonnucleus interactions.

Summary prepared by

L. Voyvodic, May 1976 
Search for Massive Neutral Particles

C.A. Ayre, H.R. Gustafson, I.W. Jones, M.J. Longo,

P.V. Ramana Murthy, (University of Michigan)

It is proposed to search for new massive neutral particles in the M3 and M4 beams of the Meson Laboratory. The search would be sensitive to neutral particles with mass $\gtrsim 2 \mathrm{GeV}$ and with proper lifetimes $\geq 10^{-7}$ sec. The masses of the particles would be determined by measuring their time-of-flight relative to the R.F. and their energy with a total absorption calorimeter.

There are at present many discussions about the possible existence of particles with new quantum numbers, such as charm or color. Most guesses suggest that their masses would be of. the order of several $\mathrm{GeV}$ and their lifetimes of the order of $10^{-13} \mathrm{sec}$. However, it is quite possible that new particles exist with a combination of quantum numbers such that much longer lifetimes would be possible. Furthermore no previous experiment could have detected neutral heavy particles with $10^{-8}<\tau<10^{-3}$ sec.

The apparatus would be principally the Michigan calorimeter, which is presently located at the end of the M3 line for use by Experiment \#248. They plan to tune up the calorimeter in the M3 beam and take actual data in the M4 beam, which should allow a more sensitive test because of the reduced neutron background.

They request approximately 2 months for testing in the M3 beam line (with some low intensity runs) and 2-3 weeks of data taking in the M4 line. 


\section{A PROPOSAL FOR A DETAILED STUDY OF DI-MUON PRODUCTION}

K. Anderson, D. Bintinger, J. Curry, J. Pilcher, J, Sidles (University of Chicago); G. Sanders, A.J. Smith, J.J. Thaler (Princeton University)

It is proposed to measure the detailed character of di-muon production in hadron-nucleon collisions (hN $\rightarrow^{+}+\mathrm{H}^{-}+$anything) In particular they will study the di-muon mass distribution down to low masses, the angular distribution and density matrix elements as a function of bombarding energy and beam particle species. The experiment will use the Chicago cyclotron spectrometer in the Muon Laboratory.

Experiment E-70, E-100A, and E-184 have recently observed direct muon production in nucleon-nuclei collisions. The yields are $10^{-4}$ of the pion yield, the ratio being apparently independent of $p_{t}$ and $\theta \mathrm{cm}$. These directly produced muons could come from the decay of vector mesons or from new fundamental phenomena Since the single prompt muons may be one of a pair, it is important to study the direct production of di-muons.

The experimental equipment consists of a heavy target (later on hydrogen and deuterium targets will be used), a hadron shield, a magnetic spectrometer with large acceptance and muon identifier The magnetic spectrometer and muon identifier are those of the E-98 muon scattering experiment. Additional proportional chamber are used between the target and hadron shield to allow a mass resolution which is unaffected by scattering in the shield. The triggering will be done in two modes:

(i) A non-selective trigger to study all muon pairs traversing the spectrometer and

(ii) A selective mode where low di-muon masses and low pt pairs are not recorded.

The only background which cannot be easily rejected is that of two muons originating from two pions from the same event. This will be monitored by recording triggers having 2 muons of the same sign, and by studying the u-pair rate as a function of distance between the production point and the hadron shield. Di-muon mass resolution is expected to be better than \pm 5 \% over the range $0.0<x_{u}<0.7$. The range of production angles to be studied corresponds to $0.2<x_{\mu \mu}<1$ and $p_{t}<3 \mathrm{GeV} / \mathrm{c}$. Mass acceptance extends to $10 \mathrm{GeV}$.

They request a beam with $10^{7} \pi$ /burst at $50,100,200$ and 300 GeV. Their sensitivity with such a beam is 1 event/hour at a cross section of $10^{-3} 4_{\mathrm{cm}^{2}}$. They estimate a need for 100 hours 0 data-taking with heavy targets and 400 hours with hydrogen and deuterium targets. The actual event yield will depend on the production cross section which is unknown at this time. Should the single prompt muons be one of a pair, then several million events would be collected with the requested beam flux.

Initial running will be parasitic with a beam flux of approximately $3 \times 10^{5}$ particles/pulse. 


\section{STUDY OF THE INTERAC'ITONS OF $200 \mathrm{GeV} / \mathrm{C} \mathrm{K}^{-}$IN THE FERMILAB 15-FOOT HYDROGEN BLBBLE CHAMBER \\ Birmingham, Cambridge, Florida state J. G. Rushbrooke (Spokesperson)}

Using $300 \mathrm{GeV} / \mathrm{c}$ protons incident on a target high momentum negative secondaries, being mainly $\Sigma^{-}$hyperons, will be focussed onto a second target in Enclosure 100 using a system of high gradient pulsed quadrupole magnets. The $\mathrm{K}^{-} / \pi^{-}$ratio obtained from the second target should be much higher than that obtainable at the first and is expected to be $20 \%$ or more at the bubble chamber. The number of pictures requested is 100,000 with approximately one incident $\mathrm{K}^{-}$per picture. The particle tagging system using the upstream P.W.C's and Cerenkov counters would be essential.

In a fiducial volume of length 2 metres slightly more than $10,000 \mathrm{~K}^{-\mathrm{p}}$ interactions would be obtained. As this is to be regarded as a survey experiment all the events would be measured and all aspects of $\mathrm{K}-\mathrm{p}$ interactions at $200 \mathrm{GeV} / \mathrm{c}$ studied. Special emphasis would be given to the study of processes involving visible strange particle decays, for which the 15 foot bubble chamber is an excellent detector.

Summary updated by

J. G. Rushbrooke, January 1976 
STUDY OF THE INTERACTIONS OF $200 \mathrm{GeV} / \mathrm{C} \mathrm{K}^{+}$IN THE FERMI LAB 15-FOOT HYDROCLN BUEBLE CHAMBER

Bonn, Cambridge, CERN, Aachen

J.G. Rushbrooke (Spokesperson)

Using $400 \mathrm{GeV} / \mathrm{c}$ incident protons and a polyethylene particle filter in enclosure 100 it should be possible to make a $200 \mathrm{GeV} / \mathrm{c}$ particle beam containing at least $20 \%$ positive kaons. The number of pictures requested is 100,000 with approximately one incident $\mathrm{K}^{+}$per picture. The particle tagging using the upstream P.W.C.'s and Cerenkov counters would be essential.

In a fiducial volume of length 2 metres a little over $10,000 \mathrm{~K}^{+} \mathrm{p}$ interactions would be obtained. As this is to be regarded as a survey experiment all the events would be measured and all aspects of the $\mathrm{K}^{+} \mathrm{p}$ interactions studied. Special emphasis would however be given to processes involving visibel strange particle decays for which a large bubble chamber is ideal.

Summary upaated by

J. G. Rushbrooke, January 1976 


\section{A SEARCH FOR DIRECT MUON PRODUCTION}

IN THE FORWARD DIRECTION

D. Buchholz (Caltech); R.P. Johnson (Fermilab); R.L. Sumner (Princeton); O. Fackler (Rockefeller); H. Frisch, M. Shochet (Chicago)

The experimenters propose to search for direct muon production in the forward direction from $300 \mathrm{GeV}$ protons incident on a heavy nuclear target. They would use the first stage of the MI beam as a diffracted proton beam and the second and third stages of MI as a spectrometer. This would allow measurements of the direct muon to pion ratio at values of $x$ between 0.3 and 0.75 , at a fixed and small value of $p_{t}$ $(<0.3 \mathrm{GeV} / \mathrm{c})$.

The recent measurements at a number of laboratories gave a relatively abundant production of directly produced leptons at large angles with a ratio of $10^{-4}$ of the pion rate, the rate being essentially independent of energy and transverse momentum (at large $p_{t}$ ). It is possible that these leptons come from the dileptonic decays of vector mesons, such as the $\rho, \omega$ and $\phi$. If the direct lepton $/ \pi$ ratio were equal to $10^{-4}$ also at low $p_{t}$ for a wide range of $x$, the vector mesons would have to be produced at least as copiously as pions in strong interactions. The purpose of this search would be to extend the measurements of direct muon production over a wide range in $x$.

The first stage of the MI beam would be transformed into a diffracted proton beam with an intensity of $5 \times 10^{8}$ protons/ pulse. The beam would strike a variable density target followed by a steel muon filter. The second and third stages of the beam line would be used as a spectrometer with $\Delta \Omega \Delta \mathrm{p} / \mathrm{p}=5 \times 10^{-8}$ steradians. A simple muon detector would be located at the end of the spectrometer. Event rate calculations show approximately 800 direct
muons detected per hour at $x=0.5$ and assuming $\mu$ direct $/ \pi=10^{-4}$.

Backgrounds are expected to come from muon decays of $\pi^{\prime} s$ and $\mathrm{K}^{\prime} \mathrm{s}$ inside the target and absorber, muons from hadron punch through, and muon contamination in the beam. The first source can be measured by varying the target density, the second and third by using a cerenkov counter downstream of the target to measure pions and muons at that location.

It is claimed that the experiment would involve only a minor perturbation on the MI beam line and the addition of only a few items of equipment. The request for running time includes approximately one week for testing and one week for data-taking.

Summary prepared by

A.F. Greene, September 1974 
MULTIPARTICLE PRODUCTION IN NUCLEON-NUCLEUS

COLLISIONS AT $400 \mathrm{GeV}$

F. Fumuro, R. Ihara, T. Ogata and Y. Yukimasa (Kwansei Gakuin University)

The object of our experiment is to investigate the mass number (A) dependence of various quantities such as absorption cross section, multiplicity, inelasticity, angular distribution etc. at $400 \mathrm{GeV}$ proton-nucleus interactions.

To investigate the nucleon-nucleus interactions, emulsion chambers are used. The emulsion chamber consists of nuclear emulsion and various metals piled up in an alternative way. The total size of this chamber is $10 \mathrm{~cm} \times 10 \mathrm{~cm} \times 2.5 \mathrm{~cm}$. Four chambers are prepared for this experiment.

The chambers are exposed to proton beam in the vertical direction to the emulsion plane.

Intensity need is $10^{5}$ particles $/ \mathrm{cm}^{2}$.

Summary prepared by

J. Lach, May 1976 
MEASUREMENT OF DI-MUON EVENTS IN THE MESON AREA

D. Eartly, G. Giacomelli (Fermilab); K. Pretzl (Max-Planck)

The experimenters propose to study the direct dimuon production with the double arm muon-range telescope located in the hall downstream of the target train in the Meson Area. Each telescope consists of six scintillation counters. The telescopes are at present looking at the Meson Area beam dump at an angle of $76 \mathrm{mrad}$ with respect to the incoming proton beam, covering a transverse momentum range of $1.4<\mathrm{p}_{\mathrm{t}}<2.7 \mathrm{GeV} / \mathrm{c}$ and a dimuon mass range of $2.5<\mathrm{m}_{\mu \mu}<5.2 \mathrm{GeV}$.

For this study they request two periods of running time totaling 1.5 hours, with no Meson Area target. If successful they may wish to move the range telescopes to other angles and would require two extra runs of half an hour each.

Summary prepared by

G. Giacomelli, September 1974 


\section{PION - DEUTERON INTERACTIONS AT $400 \mathrm{GeV}$}

J. Erwin, J. Klems, W. Ko, R. Lander, D. Pellett, P. Yager (Univ. of California, Davis); L. Dunn, H. Lubatti, K. Moriyasu, W. Podolsky (Univ, of Washington); J. Bartke, A. Eskreys, P. Malecki, K. Zalewski (Cracow); R. Sosnowski, M. Szczekowski, A. Wroblewski, A. Zieminski (Warsaw)

It is proposed to take 50,000 pictures of the 30 -inch deuterium filled bubble chamber exposed to a $400 \mathrm{GeV}$ negative pion, beam. Due to the manner in which the accelerator and the beam are operated, it is likely the actual energy will be at about $375 \mathrm{GeV}$. Use will be made of the downstream PWC hybrid spectrometer. The purpose of this experiment is to utilize the properties of the deuteron to study reactions which cannot easily be studied on protons. This may include: 1) inclusive reactions such as $\pi^{-}+n+\pi^{ \pm}+$anything, 2) diffractive dissociation of the neutron, and 3) diffractive dissociation of pions on deuterons.

Summary prepared by

A. F. Greene, June 1975 
CRACOW EMULSION EXPOSURE TO 200 GEV PIONS

$$
\text { Institute of Nuclear Physics, Krakow (Poland) }
$$

Summary:

In the last two years we were dealing with the interactions of $67 \mathrm{GeV}, 200 \mathrm{GeV}$ and about $3000 \mathrm{GeV}$ protons with nuclei of photographic emulsion. The results of the analysis performed have been summarized in a few publications listed below. These results contain new informations about some regularities in the rapidity distributions, as a function of the target nucleus excitation. It seems for us very interesting to perform similar analysis with primary pions. It may occur that this analysis applied to the final states in proton - nucleus and in pion nucleus interactions may easily detect differences between them. Therefore we would like to expose to the $200 \mathrm{GeV}$ pion beam in NAL two stacks of Ilford G5 stripped stripped pellicles.

Emulsions will be prepared in plastic containers $/ 5 \mathrm{~cm} \times 5 \mathrm{~cm} \times$ $10 \mathrm{~cm} /$, with engraved straight linc, which will be used for optical adjusting of the stack parallel to the beam / $\pm 10 \mathrm{mrad} /$. The optimum density of pion tracks should be between $5 \times 10^{4} /$ $\mathrm{cm}^{2}$ and $3 \times 10^{5} / \mathrm{cm}^{2}$ at the entrance side of the stack.

\section{References :}

J. Babecki et al. Physics Letters B $47268 / 1973 /$

J. Babecki et al. Acta Physica Polonica B 5, 315/1974/

R. Holynski et al. Acta Physica Polonica B 5, 321/1974/

J. Babecki et al. Physics Letters B 52, 24771974/ 
STUDY OF THE ELECTRON-PHOTON CASCADE SHOWER IN LEAD ABSORBER

Kobe University, Konan University, Nagoya University, Saitama University, University of Tokyo, Utsunomiya University, wasede University

S. Dake (Spokesman)

The purpose of our experiment is to study the three-dimensional behaviour of electron-photon cascade shower in lead, using nuclear emulsion plates inserted at various depth in lead absorber. Measuring the shower electrons on each shower in nuclear emulsion with a microscope, the following informations are obtained: (a) lateral and longitudinal development of shower electrons in lead absorber, (b) fluctuations of shower development at various depth and (c) effects of the inhomogeneous media on the shower development. These results are compared with the three-dimensional cascade theory, the Monte Carlo calculations and the results obtained with detectors such as ionization chambers, scintillation probes, X-ray films and thermoluminescent dosimetry.

We are preparing 30 units of detectors. The geometrical size of each detector is about $14 \mathrm{~cm} \mathrm{x} 12 \mathrm{~cm} \times 10 \mathrm{~cm}$, and it is constituted of nuclear emulsion plates and lead plates (thickness of $0.5 \sim 5.0 \mathrm{~mm}$ ) which are piled up alternately. The parallel and mono-energetic electron beams are irradiated perpendicular or oblique to the front of each shower detector. In order to suppress the room background, the whole of the detector must be surrounded by lead bricks of thickness of about $10 \mathrm{~cm}$. Following are the summary of the conditions required in this experiment.

1. Beam; parallel and mono-energetic electron beams,

2. Energy of the irradiated beam; several points between about $50 \mathrm{GeV}$ and the maximum energy,

3. Accuracy of the beam energy; better than $\sim 3 z$ (absolute) and $\sim \pm 3 \%$ (relative),

4. Amounts of irradiation; 5-10 electrons $/ \mathrm{cm}^{2}$ on each detector,

5. Running time; about two hours,

6. Shield material; lead bricks (about $0.5 \mathrm{~m}^{3}$ in total volume) enough to shield each detector. 


\section{-194- $\quad$ 15-Foot $\pi^{+} \& \mathrm{p}-\mathrm{p}$ @ $150 \# 341$}

INTERACTIONS OF $\pi^{+}$MESONS AND PROTONS IN HYDROGEN

W. Ko, J.H. Klems, R.L. Lander, D.E. Pellett, P.M. Yager (Univ. of California, Davis); R.P. Ely, G. Gidal, W.B. Michael (Lawrence Berkeley Laboratory)

The exposure of 25,000 pictures at $150 \mathrm{GeV} / \mathrm{c}$ will be used to explore the usefulness of the large geometric volume chamber for hadron physics. In particular the 15-foot chamber offers improved momentum resolution for charged particles allowing a detailed study of correlations. The large volume makes possible the detection and measurement of the $\gamma$-rays fro:n $\pi^{\circ}$ decay or decays of other particles. Thus, direct measurement of the momentum of neutrals is possible, so there is the possibility of kinematic constraints when such neutral particles are present. These conditions make possible the study of the following topics:

- correlation studies.

- neutral particle multiplicities by direct observation.

- inclusive distributions of vector mesons, including in particular $\rho^{\circ}$ and $\omega^{\circ}$.

- inclusive distributions and correlations of other neutral particles ( $\mathrm{K}^{\circ}$ and $\Lambda^{\circ}$ and $\bar{\Lambda}^{\circ}$ ).

- heavy resonance mass spectroscopy, since all decay products, charged and many neutral, can be measured.

The photographs will be scanned and pre-digitized at Davis and LBL, and measured at LBL. The track reconstruction and fitting programs are presently being developed. We will develop both TVGP and Hydra-Thresh for the 15-foot geometry. 
PROPOSAL TO STUDY NEUTRAL PION PRODUCTION IN $200 \mathrm{GeV} / \mathrm{c} \pi \pi^{-} \mathrm{P}$ INTERACTIONS IN THE

15-FOOT BUBBIE CHAMBER WITH A TRACK-SENSITIVE TARGET

R. Louttit and J. Sondericker(BNL); N.N. Biswas, N.M. Cason, V.P. Kenney, W.D. Shephard, J.M. Bishop (Univ. of Notre Dame)

It is proposed to study neutral pion production in 200 $\mathrm{GeV} / \mathrm{c} \pi^{-} \mathrm{p}$ interactions in a track-sensitive liquid hydrogen target mounted in the 15-foot bubble chamber with neon/ hydrogen filling. The experimenters are interested in exclusive channels in which no neutral pions are produced, single-inclusive production of $\pi^{\circ} ' s$, two-particle inclusive production and correlations of $\pi^{\circ} \pi^{\circ}$ and $\pi^{\circ}$ pairs, and cluster-model analyses of events with no missing neutral pions. They request a preliminary engineering run of $\sim 10,000$ pictures and a final data run of 200,000 pictures with an average of 4 tracks/picture through the target. The tracksensitive target, with instrumentation and control system, will be supplied by the experimenters.

Summary prepared by

V. Paul Kenney, January 1976 


\section{PROPOSAL TO STUDY NEUTRAL PARTICLE FRODUCTION \\ IN $250 \mathrm{GEV}$ PP INTERACTIONS IN THE FERMILAB 15-FOOT BUBBLE CHAMBER}

K. Jaeger (ANL); R. Ammar, R. Davis, L. Loos, R. Stump and N. Kwak (Univ. of Kansas); A. Brody, R. Engelmann, J. LeeFranzini, J. Hanlon and T. Kafka (SUNY at Stony Brook); J. Canter, F.T. Dao, A. Mann and J. Schneps (Tufts Univ.)

A 25,000 picture exposure is proposed of the hydrogen-filled Fermilab 15-foot bubble chamber to a $250 \mathrm{GeV}$ proton beam.

The main physics objectives are:

1. To study the dependence of neutral particle production $\left(\pi^{\circ}, \mathrm{K}^{\circ}, \Lambda\right)$ on the charged multiplicity in diffractive and non-diffractive processes.

2. To measure integrated correlations $\left(f_{2}^{ \pm 0}\right.$ and $f_{2}^{00}$ ) for neutral pions in a "clean" experiment.

To make these measurements reliably requires neutral particle detection in both $\mathrm{CM}$ hemispheres and the $\mathrm{CM}$ symmetry of the PP system. For example, $f_{2}^{0}$ will be determinea to \pm 0.2 .

The event analysis of the neutral secondaries together with the measurement of the slow laboratory protons in $\mathrm{pp} \rightarrow \mathrm{p}_{\text {slow }}+$ $x$ for the aistinction between diffractive and nondiffractive events will be done in $1 / 2$ year.

Further objectives are:

3. To measure differential neutral-charged correlations.

4. To measure multiplicities, spectra and correlations ("inclusive program") inside the mass $\mathrm{M}_{\mathrm{X}}$ in $\mathrm{pP} \rightarrow \mathrm{P}_{\text {slow }}+\mathrm{x}^{-}$.

Surmary prepared by

R. Engelmann, October 1974 


\section{PROPOSAL TO SUEVEY CENTRAL COLLISIONS IN THE REACTION $\overline{\mathrm{p}}+\mathrm{p} \rightarrow$ MESONS BETWEEN 30 AND $60 \mathrm{GeV} / \mathrm{C}$ \\ IN THE 30" HYDROGEN BUBBLE CYAMRER AT N.A.L.}

V.E. Barnes, D.D. Carmony, R.S. Christian, C.R. Ezell, J.A. Gaicios, I.J. Gutay, R.L. McIlwain, A.T. Laasanen, P. Leong, D.H. Miller, T.R. Palfrey, R.B. Willman

(Purdue University); F. Turkot (Fermilab)

This is a detailed survey of antiproton interactions with particular emphasis on the annihilation process.

Annihilation interactions could give an insight into the behavior of hadrons at small distances and shed light on the detailed structure of the nucleon. This physics may well be closely related to the high $p_{t}$ phenomena and to the recent results on $e^{+} e^{-}$annihilation both of which have exhibited unexpected features which may indicate a common underlying structure. This study of central collisions is ideally suited to the bubble chamber since the cross section is high and the interactions are dominated by high multiplicities. The choice of beam momentum is such that $\sqrt{\mathrm{s}}(\sqrt{\mathrm{s}}=7.9 \mathrm{GeV})$ centers on the energy range of SPEAR; and such that the annihilation cross section is large enough to be accessible to bubble chamber study.

The 30" hydrogen bubble chamber would be used in an enriched antiproton beam at $30 \mathrm{GeV} / \mathrm{c}$. The incident antiprotons are tagged and the downstream hybrid system is used in analyzing the interaction products. With $3 \times 10^{5}$ antiprotons traversing the chamber, $19 \times 10^{3}$ interactions are expected with $2.8 \times 10^{3}$ of these being annihilation events. The expected number of photographs is $100 \mathrm{~K}$. 
PROPOSAL TO STUDY MULTIPARTICLE PRODUCTION IN $100 \mathrm{GeV} / \mathrm{C}$ ANTI-PROTON-DEUTERIUM INTERACTIONS WIITH THE FERMILAE 30-INCH BUBBLE CHAMBER

Stockholm-Liverpool-Vanderbilt

G. Ekspong (Spokesperson)

The experiment will take 100,000 pictures in the deuterium filled 30-inch bubble chamber and use the downstream PWC system. The beam will bc a tagged antiproton beam with $100 \mathrm{GeV} / \mathrm{c}$ momentum. The analysis will include the following topics:

1. Determination of $\bar{p}$ cross sections and multiplicities. Comparison of the features of $100 \mathrm{GeV} / \mathrm{c} \mathrm{pp}, \overline{\mathrm{p} p}$ and $\mathrm{pn}$ reactions.

2. An s-dependent analysis of the pn reactions making use of the data at lower energies.

3. Study of the $\pi^{\circ}$ production and two-particle correlations.

4. Study of the diffractive systems in $n \rightarrow p \pi^{-}$and $n \rightarrow p \pi_{-}^{-} \pi_{-}^{+} \pi_{+}^{-}{ }_{-}^{-}$ which could be obtained in fits to $\overline{\mathrm{p}} \mathrm{n} \rightarrow \overline{\mathrm{p}} \mathrm{p} \pi^{-}$and $\overline{\mathrm{p}} \mathrm{p} \rightarrow \overline{\mathrm{p}} \mathrm{p} \pi^{-} \pi^{+} \pi^{-}$

5. The fragmentation processes in $n \rightarrow \vec{p} \pi^{-}$and $n \rightarrow \vec{p} \Lambda^{\circ}$ will add to the understanding of the fragmentation of the nucleon. The study of the corresponding fragmentations of the proton $\mathrm{p} \rightarrow \mathrm{p} \pi$ has given interesting results.

6. Properties of the multiplicity distributions. There is a marked difference in the s-dependence of for instance the $\langle\mathrm{n}\rangle / \mathrm{D}$ ratio for $\mathrm{pp}$ and $\overline{\mathrm{p}} \mathrm{p}$ reactions. A study of the s-dependence of the $\langle n\rangle / D$ ratio for $\bar{p} n$ reactions would contribute to the understanding of this feature.

7. The possible existence of a $\Delta-\Delta$ component in the deuteron wave function. It has been suggested that a way to test such a picture is to study inelastic reactions on the deuteron. It is a great advantage to have access to the data from the $\bar{p} p \rightarrow \bar{p} p \pi^{+} \pi^{-}$reaction in order to be able to estimate the kinematical background.

8. The strange particle cross section is rising in $\mathrm{pp}$ interactions being $\sigma(\Lambda)=3.2 \pm 0.4, \sigma(\mathrm{KO})=9.2 \pm 1.0$ and $\sigma(\bar{\Lambda})=0.23 \pm 0.10 \mathrm{mb}$ at $100 \mathrm{GeV} / \mathrm{c}$. Similar cross sections in $\bar{p}$ will make a fairly detailed study of the production of $\mathrm{K}^{\circ}, \Lambda^{\circ}$ and charged hyperons possible. Antiproton annihilations to kaons are likely to be the best reactions to search for the production of charmed mesons. 
SEARCH FOR HEAVY, SHORTLIVED PARTICLES

G. Ekspong, S. Nilsson, N. Yamdagni (Stockholm Univ., Sweden)

The aim is to search for new, possible charmed particles with lifetimes around $10^{-13} \pm 1 \mathrm{sec}$.

Nuclear emulsions will serve as target and detector. The signal will be a secondary interaction (neutral decay, charged decays of several topologies) close to the primary protonnucleus vertex.

The distances will be measured in microscopes. After background elimination the remaining signals should show a distance distribution which is exponential in the first approximation. From this, the existence of new particles and their lifetime can be investigated.

Beam: $400 \mathrm{GeV}$ protons in the Neutrino Area.

Flux: $\quad\left(2-3 \times 10^{5}\right.$ protons $/ \mathrm{cm}^{2}$ over about $10 \mathrm{~cm}^{2}$

Emulsion stacks: One stack with entrance area exposed equal to $30 \mathrm{~cm}^{2}$.

Summary prepared by

G. Ekspong, January 1976 
D. Carey, R. Rammerud, D. Ritchie, J. Sauer, R. Shafer, D. Theriot, J.K. Walker (Fermilab); J. Johnson (Northeastern); F.E. Taylor (Northern Illinois University)

It is proposed to study elastic scattering of $\pi^{ \pm}, \mathrm{K}^{ \pm}, \mathrm{p}$, and $\bar{p}$ at energies of from 50 to $400 \mathrm{GeV}$ and over a $t-r$ ange of from 2 to $15(\mathrm{GeV} / \mathrm{c})^{2}$ or to a differential cross section limit of $10^{-37} \mathrm{~cm}^{2} /(\mathrm{GeV} / \mathrm{c})^{2}$. It is emphasized that measurements of the elastic cross sections over these kinematic ranges are of fundamental importance, possibly providing information on the mechanism of rising total cross sections. Tests can also be made of models which attempt to relate the dynamics of elastic and inelastic inclusive scattering. It is also mentioned that the apparatus could be useful for studies of backward scattering or of inclusive reactions.

The unseparated incident beam would have an intensity of approximately 109 particles/pulse and because of the high intensity would not have any identifying counters. The beam is incident on a 16" liquid hydrogen target. The spectrometer consists of two arms and is designed for large acceptance to be sensitive to the lowest possible cross sections. The forward arm contains a sweeping magnet, 6" $\times 3 " \times 72 "$, with $18 \mathrm{kG}$ to protect the front proportional wire chambers. This is followed by the main momentum analyzing magnet $6.5^{\prime \prime} \times 9.5^{\prime \prime} \times 20^{\prime}$ with $18 \mathrm{kG}$. Proportional wire chambers and scintillation hodoscopes are distributed throughout the system. Particle identification is accomplished using the threshold Cerenkov counters; $\mathrm{C}_{1}$ to detect pions is $65 \mathrm{~m}$ long and $c_{2}$ to detect pions and kaons is $25 \mathrm{~m}$ long. The momentum resolution in this arm will be $\leqslant 18$ and angular resolution at $100 \mathrm{GeV}$ will be $\pm 0.25 \mathrm{mr}$.

The recoil arm will have a magnet $24 " \times 15^{\prime \prime} \times 60 "$ with $15 \mathrm{kG}$. Momentum and angular resoltuion are expected to be $\leqslant 1.5 \%$ and $\pm 2.5 \mathrm{mr}$ respectively. Triggering is to be done at three levels. The first involves a scintillation hodoscope coincidence, the second a hardware matrix of hodoscope information, and the third a hardware processor of proportional chamber information.

Running time for the first phase of the experiment is described in the proposal. The elastic cross sections would be measured at $200 \mathrm{GeV}$ for $2<|\mathrm{t}|<13(\mathrm{GeV} / \mathrm{c})^{2}$, requiring 600 hours, and at 100 and $400 \mathrm{GeV}$ for $3.5<|\mathrm{t}|<7$ (Gev/c) 2 , requiring 200 hours. Also, 200 hours are requested for testing. Further studies of the s-dependence and measurements to the highest $|t|$-values will await Phase II of the experiment. 
PROPOSAL TO STUDY SCATTERING OF MUONS FROM PROTONS AND DEUTERONS AT MOMENTUM TRANSFERS UP TO $\mathrm{q}^{2}=150(\mathrm{GeV} / \mathrm{C})^{2}$

T. Loomis, F.M. Pipkin, A. Sessoms, L. Verhey, R. Wilson (Harvard); W. Francis, T. Kirk (Univ. of Illinois)

It is proposed to extend the measurements of Experiment \#98 on inelastic scattering by protons and deuterons to $\mathrm{q}^{2}=150$ $(\mathrm{GeV} / \mathrm{c})^{2}$. For this they require a higher momentum muon beam and a much longer hydrogen (deuterium) target. Besides allowing higher $\mathrm{q}^{2}$ values, these changes will also allow considerably higher rates on several interesting hadronic channels at moderate momentum transfers.

They plan to add a 10 meter long (130 liter) liquid hydroger. target in front of their present $1.2 \mathrm{~m}$ target and have both filled with $\mathrm{H}_{2}$ or $\mathrm{D}_{2}$ or one with $\mathrm{H}_{2}$ and the other with $\mathrm{D}_{2}$. A set of proportional counters will be placed between the two targets. It is not planned to study the hadrons escaping from the sides of the $10 \mathrm{~m}$ target. The trigger will be basically the same trigger used by Exp. \#98: an incident beam muon must be found in a scattering hodoscope without having any beam halo particle nor an undeflected muon forward.

They request $0.6 \times 10^{11}$ muons at each of $150 \mathrm{GeV}$ and $300 \mathrm{GeV}$ energies and each of $\mathrm{H}_{2}$ and $\mathrm{D}_{2}$ targets. Assuming a beam of $2 \times 10^{6}$ muons per pulse inside a 4 " diameter they estimate a need for total running time of 400 hours. They would favor higher muon energies if the energy of the accelerator has been increased. 
A PROPOSAL TO STUDY $\pi^{\circ}$ AND $\eta$ INCLUSIVE PRODUCTION WITH INCIDENT $\pi^{-}$IN THE TRIPLE REGGE REGION

Caltech, LBL, BNL

R. Kenney (spokesperson)

It is proposed to measure the inclusive reactions

$$
\begin{aligned}
& \pi^{-} p \rightarrow\left(\pi^{\circ}, \eta^{\circ}, \omega, \ldots\right) x^{\circ} \quad, x^{\circ} \rightarrow \text { charged + neutrals } \\
& \pi^{-} \mathrm{p} \rightarrow\left(\pi^{\circ}, \eta^{\circ}, \omega, \ldots\right) \mathrm{x}^{\circ}, \mathrm{x}^{\circ} \rightarrow \text { neutrals }
\end{aligned}
$$

in the essentially unexplored kinematic region $0 \leqslant|t| \leqslant 3(\mathrm{GeV} / \mathrm{c})^{2}$ (or $p_{t} \leqslant 1.7 \mathrm{GeV} / \mathrm{c}$ ) and $0.8 \leqslant x \leqslant 1$. This is a further continuation of the work begun in Experiment \#11l (study of exclusive $\pi$ charge exchange) and Experiment \#268 (production of $\pi^{\circ}, n$, etc. at $p_{t}$ up to about $5 \mathrm{Gev} / \mathrm{c}$ ).

The purposes of the newly proposed work are: (1) to measure the $\rho$ and $\mathrm{A}_{2}$ Regge trajectories to larger $t-v a l u e s$ than can be reached in exclusive $\pi^{-}$charge exchange; (2) to obtain data in the triple Regge region for comparison with theory for reactions involving relatively simple exchanges and where the theory makes unambiguous predictions, and (3) to study the relation between high $-\mathrm{p}_{\mathrm{t}}$ and low $-\mathrm{p}_{\mathrm{t}}$ phenomena.

The experiment would utilize the $M 2$ beam in the Meson Area with $\pi$ at 100,150 , and $200 \mathrm{GeV} / \mathrm{c}$. The experimental apparatus basicly consists of the photon detector utilized for the other experiments with the addition of a charged particle hodoscope (also used in Exp. \#268). Two set ups would be required for this experiment, one with the photon detector system on the beam line and the other with the system displaced to gne side. The respective $t$-regions covered are $1<|t|<3(\mathrm{GeV} / \mathrm{c})$ and $|\mathrm{t}|<2(\mathrm{GeV} / \mathrm{c})^{2}$.

The running time request is for approximately 5 weeks (or about 500 hours) in two periods. 


\section{$\mathrm{K}^{\circ}$ REGENERATION AT THE HIGHEST FERMILAB ENERGIES}

S. H. Aronson, R. H. March, L. G. Pondrom (Univ. of Wisconsin); J. Roehrig, V. L. Telegdi, B. Winstein (Univ. of Chicago); H. Hofer (Swiss Fed. Inst. of Tech.)

It is proposed to measure $\mathrm{K}^{\circ}$ regeneration in hydrogen and carbon in a short neutral beam where the $\mathrm{K}_{\mathrm{S}}^{O}$ component is still present. The experiment will determine the magnitude of the regeneration parameter, $|\rho|$, and its phase, $\phi \rho$, from 150 to 300 $\mathrm{GeV} / \mathrm{c}$ with incident proton energies of $400 \mathrm{GeV}$.

In $\mathrm{K}^{\circ}$ regeneration experiments one measures the regeneration parameter $\rho$ and by this determines the $K^{\circ}-\bar{K}^{\circ}$ total cross section difference. Experiment $\# 82 / 425$ by this group has made measurements up to about $120 \mathrm{GeV} / \mathrm{c}$ using the $\mathrm{M4} \mathrm{Kf}$ beam in the Meson Area. Difficulties occur in obtaining sufficient statistics in regeneration measurements when going to higher energies with a $\mathrm{K}_{\mathrm{L}}^{\mathrm{O}}$ beam. This happens when the magnitude of the regeneration parameter, $\rho$, becomes comparable with $n_{+-}$, the decay parameter for the CP-violating process $\mathrm{K}_{\mathrm{L}}^{\mathrm{O}} \rightarrow \pi^{+} \pi^{-}$. One can avoid this difficulty by performing the regeneration measurements in a beam where there is a sizable fraction of $\mathrm{K}_{S}^{O}$. Then one can measure the interference of $\rho$ and the original $k \&$ component of the beam.

The experiment would utilize a $\mathrm{K}^{\circ}$ beam from the magnetized beam dump also used for the neutral hyperon experiments. This is located in the $M 2$ beam of the Meson Area, but another similar $\mathrm{K}^{\circ}$ beam could be constructed in another location such as the Proton Area. The $\mathrm{K}^{\circ}$ detection spectrometer would consist of wire proportional chambers or drift chambers and an analyzing magnet with a gap of $0.2 \mathrm{~m} \times 0.6 \mathrm{~m}$. A $7.2 \mathrm{~m}$ liquid hydrogen regenerator (currently in use in E-425) is required. This regenerator will have to be moved from a location close to the production target to further downstream in order to study the interference as a function of distance. The extremities of the regenerator location are $50 \mathrm{~m}$ and $200 \mathrm{~m}$ downstream of the target.

The running time request includes 1,200 hours for testing and 1,800 hours for regeneration measurements from carbon and liquid hydrogen with a $400 \mathrm{GeV}$ primary proton energy. There is also interest in measurements at the highest proton beam energies. 


\section{VERY STRANGE HYPERON DECAYS}

C. Ankenbrandt, M. Atac, R. Brown, S. Ecklund, P. Gollon,

A. Lennox, J. Lach, J. MacLachlan, A. Roberts (Fermilab); J. Johnson, R. Majka, J. Marx, P. Nemethy, J. Sandweiss (Yale)

It is proposed to pursue a comprehensive program to study decays of the charged hyperons with an emphasis on the properties of the $\Omega^{-}$. While most of their effort will be devoted to decays of negative hyperons or lambdas coming from $\equiv^{-}$decay they hope to spend some effort studying leptonic decays of the $\Sigma^{+}$.

The apparatus consists of the equipment needed for Experiment \#97. The addition of superconducting quadrupole magnets to the magnetic channel to enhance the intensity of the hyperon beam is discussed in the proposal and interpreted to be useful also to the goals of Experiment \#97. The charged hyperons can be identified using two separate methods: a high resolution gas cerenkov counter and a spectrometer to observe the decay products. For this new proposal there would be the addition of detectors for electrons and possibly gamma rays.

1011 The running requirements are for a beam of approximately protons/pulse at $300 \mathrm{GeV} / \mathrm{c}$. About 600 hours of beam time will be needed for the data-taking part of the experiment. It is also asked that this study of hyperon decays be carried out immediately following Experiment \#97. 


\section{SURVEY FOR EXOTIC PARTICLES IN THE MI BEAM}

BNL, Fermilab, Rockefeller Univ. W.F. Baker (Spokesperson)

As a continuation of their beam survey in the Ml beam line, they propose to perform a survey for exotic particles, that is the production of $\bar{d}, \bar{t}, \overline{\mathrm{H}}_{\mathrm{e}} 3$, search for $\overline{\mathrm{H}}_{\mathrm{e}} 4$ and heavy mass particles with charges -1 and -2 , and search for heavy quasistable muons. For these purposes they shall use the equipment of Experiment \#104 already set-up in the Ml beam.

In particular they will use extensively the two Brookhaven gas Cerenkov counters and will be able to test their ultimate capabilities.

It has been proven at Serpukhov, Fermilab and at the ISR that the efficient production of antinuclei requires that the bombarding proton energy be considerably greater than the threshold value. The Fermilab accelerator offers the possibility of testing models of antiparticle production and establishing the existence of as yet unobserved antiruclei or heavier particles. As far as the heavy muon search, such heavy muons are required by certain gauge theories of weak interactions.

Specifically they plan to do the following searches with 300 or preferably $400 \mathrm{GeV}$ protons:

i) Detection of charge 1 particles with heavy mass. They plan to measure $d$ and $\bar{d}$ production at 60,80 and $100 \mathrm{GeV} / \mathrm{c}$. The study of $\bar{t}$ production and the search f.or heavier masses will be done at $80 \mathrm{GeV} / \mathrm{c}$ to a sensitivity of approximately 1 in $10^{10}$ pions. For this purpose two gas Cerenkov counters will be placed in anticoincidence; one will be placed on the $\bar{t}$ mass and time of flight will be used for heavier masses.

ii) Detection of antihelium nuclei. The study of $\overline{\mathrm{H}}_{\mathrm{e}}^{3}$ production and the search for $\overline{\mathrm{H}}_{\mathrm{e}} \mathrm{will}$ be done with the $\mathrm{Ml}$ beam set at a momentum of $\mathrm{p} / \mathrm{e}=40 \mathrm{GeV} / \mathrm{c}$, corresponding to $80 \mathrm{GeV} / \mathrm{c}$ for antihelium nuclei. The search will be carried out to 1 particle in $3 \times 10^{10}$ pions.

iii) Search for heavy quasi-stable leptons. For this search the first collimator of the MI beam line will be closed and the muon detector of Experiment \#104 will be used. The search will be carried out to 1 particle in 1011 pions.

They request $1-3$ weeks of stable running at $300 \mathrm{GeV}$. An additional week at $400 \mathrm{GeV}$ will be requested if the beam spill is adequate. 
MEASUREMENT OF TOTAL $v, \bar{v}$ CROSS SECTIONS AT HIGH ENERGIES

B.C. Barish, J.F. Bartlett, A. Bodek, K.W. Brown, D. Buchholz, F.S. Merritt, F.J. Sciulli, L. Stutte, H. Suter (Caltech);

E. Fisk and G. Krafczyk (Fermilab)

It is proposed to measure the total cross sections for $v$ and $\bar{v}$, with at least one muon in the final state, from 30 to $300 \mathrm{GeV} / \mathrm{c}$ with an accuracy of better than \pm 108 .

The proposed work represents a logical continuation of the research program initiated by the group associated with Exp. \#21A. Accurate total cross sections for neutrino and antineutrino interactions have been a prime goal since the construction of the Fermilab accelerator.

The participants have already measured the total cross sections for $v$ and $v$ using the dichromatic beam at 40 and $105 \mathrm{GeV}$. The present limitations are purely systematic and do not allow measurements to better than \pm 208 . In order to achieve precision of \pm 108 they need to (i) improve the dichromatic beam, (ii) change to a new larger toroidal magnet in order to have a better efficiency for detecting large angle muons, (iii) perform an accurate and extensive beam survey, and (iv) improve the flux monitors.

They plan to measure the total cross sections at 10 neutrino energies between 30 and $300 \mathrm{GeV}$. For this program they request 400 hours for beam survey with a low intensity and long spill beam and 1000 hours of data-taking with the dichromatic neutrino and antineutrino beams. This experiment should logically follow that of Proposal \#356.

Summary prepared by

G. Giacomelli, October 1974 


\section{STUDY OF DEEP INELASTIC DIFFERENTIAL DISTRIBUTIONS AT HIGH ENERGIES FOR $v, \bar{v}$ BEAMS}

B.C. Barish, J.F. Bartlett, A. Bodek, K.W. Brown, D. Buchholz, F.S. Merritt, F.J. Sciulli, L. Strutte, H. Suter (Caltech); E. Fisk and G. Krafczyk (Fermilab)

It is proposed to make a systematic study of deep inelastic cross sections using a new dichromatic v-beam and improved equipment of the Caltech-Fermilab collaboration.

The participants have already measured in Exp. \#21A the $\mathrm{x}-, \mathrm{y}^{-}$, and $\mathrm{q}^{2}$-distributions in deep inelastic neutrino-nucleon interactions

$$
\begin{aligned}
& v_{\mu}+N+\mu^{-}+x \\
& \bar{v}_{\mu}+N+\mu^{+}+x
\end{aligned}
$$

Because of the small acceptance of their toroidal magnet they had a reasonable acceptance only up to $y=0.6$ and had a considerable distortion in the $x$-distributions. The improved setup should allow reasonable acceptances to larger values of $y(y \sim 0.8), x$ and $q^{2}$ and should provide a significant step towards a quantitative study of deep inelastic neutrino and antineutrino scattering.

The apparatus will be the same of Exp. \#2lA but will have a larger toroidal magnet and an improved neutrino beam. These improvements will result in larger acceptances as well as in improved resolution for the muon momentum $\left(\Delta \mathrm{p}_{\mu} / \mathrm{p}_{\mu} \simeq 0.14\right.$ instead of the present 0.21$)$ and neutrino energy $\left(\Delta E_{\nu} / E_{\nu} \simeq 0.10\right.$ instead of the present 0.30 ).

They request 1000 hours of running time with the dichromatic $v$ and $v$ beams. In this time they should be able to collect approximately 10,000 v-events and $3,000 \mathrm{v}$-events. 
A PROPOSAL TO SEARCH FOR CHARMED PARTICLES AND MEASUREMENTS OF TWO-PARTICLE INCLUSIVE CROSS SECTIONS AT LARGE $P_{T}$

D. Jovanovic, R. Lundy (Fermilab); D. Finley, F. Loeffler,

E. Shibata, K. Stanfield (Purdue Univ);R. Loveless, D. Meyer,

R. Thun (Univ. of Michigan) reaction

They propose to search for charmed mesons in the following

$$
\text { hadron + hadron }+\underset{L}{C}+\pi K
$$

where $\mathrm{C}$ is a charmed meson. They will use a two-arm symmetric spectrometer. The $C$ meson would be identified as a narrow spike in the $\pi \mathrm{K}$ effective mass spectrum. As a byproduct they will obtain data on inclusive two-particle production as a function of the two particle momenta and types.

The possibility of a fourth "charmed" quark, suggested several years ago to provide a more symmetrical relationship between the four known leptons and the quarks, has recently received considerable attention since it allows one to construct a consistent theory of weak interactions. At present there is no direct experimental evidence for charmed hadrons. They are expected to have a mass between 2 and $10 \mathrm{GeV}$ and a lifetime of the order of 10-12 sec. Charmed mesons should have a sizeable $\mathrm{K} \pi$ branching ratio (about $108-50 \%$ ); this decay tends to give pions and kaons at large transverse momentum $\left(p_{t} \simeq m_{C} / 2\right)$.

The study of two-particle correlations at large $p_{t}$ should give information on the production mechanism (for instance on cluster production) and on the dependence of the correlation on the types of particles produced and on their $\mathrm{p}_{t}$.

The experiment is using the M2 beam, with intensities of the order of $10^{8}$ particles per burst, impinging on a heavy target. The two spectrometers will be identical; they will be aimed at \pm 100 mrad with respect to the incident beam direction. They will each employ one $8 \times 24 \times 72$ " bending magnet, three gas differential cerenkov counters and several planes of drift chambers. This set-up covers the mass range $2<\mathrm{m}_{\mathrm{C}}<10 \mathrm{GeV}$, with a mass resolution of approximately $\Delta M_{C} \approx 0.005 \mathrm{f}_{C}$, and an efficiency of the order of $10^{-3}$ of the $C$ production cross section.

They estimate a need for about 6 months of running time. 
Columbia Univ., Cornell Univ., Univ, of Hawaii, Univ, of Illinois (Urbana), Fermilab W. Lee (Spokesperson)

It is proposed to measure the invariant cross section of dimuons produced by neutrons in a nuclear target.

Direct lepton production has already been observed in both photon-nucleon and neutron-nucleon collisions using the equipment of Experiment \#87A. The neutron induced events indicate the presence of a rho meson and a $\psi(3.1)$ signal.

The experimental equipment consists of the apparatus of Experiment \#87A supplemented with a hadron calorimeter and a steel shield placed $5^{\prime}$ after the target. When it is used, the total energy of the electromagnetic and hadronic secondaries accompanying each interaction is measured. In the absence of the production of neutrinos the total energy of the event is measured. Data can also be taken without the steel; in this instance some information can be obtained about the high energy secondaries which accompany the dilepton. The $0^{\circ}$ neutral beam can be easily changed from a photon beam to a hadron beam by appropriate insertion of 6 radiation lengths of lead and removal of the deuterium filter.

It is proposed to measure dimuon production with neutrons procuced by 300 and $400 \mathrm{GeV}$ protons. In particular data would be taken:

(a) At $400 \mathrm{GeV}$ with low intensity $\left(2 \times 10^{6}\right.$ neutrons per second) to study dimuons with $\mathrm{m}_{\mu \mu}^{2}<10 \mathrm{GeV}^{2}$ and $t_{\mu \mu}<10(\mathrm{GeV} / \mathrm{c})^{2}$;

(b) At $400 \mathrm{GeV}$ with high intensity $\left(\sim 10^{7} \mathrm{n} / \mathrm{sec}\right)$ to study dimuon production with $\mathrm{m}_{\mu \mu}^{2}<25 \mathrm{GeV} / \mathrm{c}$ and $t_{\mu \mu}<20(\mathrm{GeV} / \mathrm{c})^{2}$;

(c) Running with a $300 \mathrm{GeV}$ front porch to get $300 \mathrm{GeV}$ data at the same time as the $400 \mathrm{GeV}$ running. 
G. Bunce, R. Handler, R. March, L. Pondrom, M. Sheaff

(Wisconsin); K. Heller, O. Overseth (Michigan); T. Devlin,

R. Edwards, P. Yamin (Rutgers)

Recent progress in the gauge theory of weak and electromagnetic interactions provides motivation for improving the data on hyperon beta decays. The Fermilab neutral hyperon beam is capable of producing a sample of lambda beta decays significantly larger than what has previously been possible. Current yield measurements from Experiment \#8 in the M-2 beam in the Meson Area predict $3 \Lambda \rightarrow$ pe $\nu$ events per $10^{8}$ incident protons. The experiment would be performed with the apparatus already in place for Experiment \#8. A study is being made of the feasibility of using synchrotron radiation from bending the outgoing electron in the magnetic field of the analysis magnet to identify this particle. It is proposed to add a xenon multi-wire proportional chamber behind the magnet to measure the electron and its corresponding radiated photons. It is also planned to study possibilities for producing polarized $\Lambda^{\prime} s$ (or $\bar{\Lambda}^{\prime} s$ ) from the decay of $\Xi^{\prime}$ 's (or $\bar{E}{ }^{\prime} ' s$ ). The experimental setup is compatible with that for the $\Lambda-p$ total cross section measurements to be made in $E-8$. Total requested running time is 300 hours. 5 It is estimated that in about 200 hours approximately $4 \times 10^{5} \Lambda \rightarrow$ pe $v$ decays will be observed with I,200 polarized $\Lambda^{\prime} s$ and $400 \bar{\Lambda}^{\prime} s$.

Summary prepared by

A. F. Greene, June 1975 


\section{INTERACTION OF 200-400 GeV PIONS WITH EMULSION NUCLEI \\ Piyare L. Jain (State Univ. of New York at Buffalo)}

Recently we have studied proton-Nucleon and proton-Nucleus interactions with 200 and $300 \mathrm{GeV}$ proton beams at FNAL. These studies have been confined to the multiplicities and to the angular distributions of the shower particles produced in protonnucleor: and proton-nucleus interactions. Low multiplicity observed in complex nuclei does not depend upon the size or the excitation of the target nuclei and is not expressed by the internuclear cascade models. It has been found that in the target nucleus $A$, the ratio $\mathrm{R}_{\mathrm{A}}=\left\langle\mathrm{N}_{\mathrm{S}}\right\rangle_{\mathrm{pA}} /\left\langle\mathrm{N}_{\mathrm{Ch}}\right\rangle \mathrm{pp}$, where $\left\langle\mathrm{N}_{\mathrm{S}}\right\rangle_{\mathrm{pp}}$ is the mean $\mathrm{p}-\mathrm{p}$ charge multiplicity and $\left\langle\mathrm{N}_{\mathrm{S}}\right\rangle \mathrm{pA}$ is the mean p-nucleus multiplicity in nucleus $A$ is practically constant arid increases very slowly with $\mathrm{N}_{\mathrm{h}}$ value. The scaling relation in emulsion is given by $R_{E}=a+b N_{h}$ where $a=1$ and $b=0.06$. We also find that the rapidity distributions for $p$-nucleon and p-nucleus interactions are the same in the forward airection and are slightly different in the backward direction.

We would like to use pion as a primary particle and compare the results of $(200-400) \mathrm{GeV}$ proton beams with the pion $(200-400) \mathrm{GeV}$ beams results.

Summary prepared by

P. L. Jain, March 1976 


\section{A PROPOSAL TO SEARCH FOR CHARMED PARTICLE PRODUCTION NEAR THRESHOLD}

D. Gross, D. Nitz, S. Olsen (Univ. of Rochester); K. Abe, R. Bomberowitz, K. Cohen, M. Margulies, F. Sannes (Rutgers); A. Colleraine (Florida State); I. Siotis (Imperial College)

Since the discovery of $J$ or $\Psi$ (3105) there has been speculation as to the classification of this new particle. It has been suggested that the $\Psi$ (3105) is a charmed vector singlet, member of the SU(4) symmetry group. From the known properties of the $\Psi$ there are predictions of charmed vector and pseudoscaler mesons with masses of approximately $2.2 \mathrm{GeV}$ and of charmed baryons with masses of 4-5. GeV. It is also expected that charmed mesons are produced in pairs in strong or electromagnetic interactions and that they decay into $K \pi$ final states. This proposed_experiment would carefully examine the energy dependence of the $\mathrm{K}^{-} / \pi^{-}$ratio, looking specifically for abrupt changes or anomalies possibly generated from reaching the threshold energy for production of a particle.

The experimental apparatus would consist of a spectrometer located within the Internal Target Area and utilizing the upstream rotating target. The spectrometer would consist of an $18 \mathrm{kG}$ bending magnet with a position variable from $15^{\circ}$ to $40^{\circ}$ with respect to the beam line. On either side of the magnet are scintillation hodoscopes to provide trajectory information and threshold gas Cerenkov counters. The acceptance is limited by the aperture of the rear cerenkov counter and expected momentum error, $\Delta \mathrm{p}$, is expected to be about $\pm 0.07 \mathrm{GeV} / \mathrm{c}$ at the $15^{\circ}$ angle setting. Using a rotating carbon target they expect in about one day of running to obtain lo statistics in each energy bin, variable from about $20 \mathrm{GeV}$ to the maximum energy of the accelerator. This must be multiplied by the number of angle settings. Tuning time is expected to take 2-3 weeks.

Follow-up running could include measurements using the hydrogen jet target and a coupling of this apparatus to the spectrometer under construction for Experiment \#198 to look for Kp decays of charmed baryons.

Summary prepared by

A.F. Greene, May 1975

Reviewed by

S. Olsen, January 1976 
A PROPOSAL TO SEARCH FOR THE PRODUCTION OF CHARMED MESONS IN TP INTERACTIONS

Northeastern University

D. Garelick (Spokesperson)

An experiment is proposed which searches for the production of charmed mesons, $D$, produced in the reactions

$$
\begin{aligned}
& \pi^{-} p+x^{-} p+D \bar{D} p \\
& \pi^{-} p+X^{-} p+D \bar{D}(n \pi) p
\end{aligned}
$$

Reactions(2) differs from reaction (1) in that $n$ additional pions are produced. For each event the final state proton as well as one or more of the muons from the $D$ or $\bar{D}$ decays will be measured. The experiment looks for $D^{\prime} s$ in the mass range $1.0 \leq M_{D} \leq 4.5 \mathrm{GeV}$. A total production cross section for reaction (1) plus reaction (2), $\sigma_{1}$, , of $5 \mu \mathrm{b}$ for $2 M_{D} \leq M_{X} \leq\left(2 M_{D}+1 \mathrm{GeV}\right)$ and a branching ratio for $D+\langle\mu v$ hadrons) of 0.1 is expected to give in one day of running a more than 7 standard deviation effect in the observed mass, $M_{X}$, spectrum from $\mu p$ coincidence events.

The experiment uses the existing apparatus of Experiment \#51A in addition to a $\mu$ detector yet to be installed in the $M 2$ beam line. The experiment can be carried out in a three week running period with 200 hours of beam consisting of 40 hours for testing and 160 hours for data-taking. 


\section{STUDY OF HEAVY, NARROW MESONS USING}

A MASS FOCUSING SPECTROMETER

M. A. ABOLINS, J. A. J. Matthews, R. A. Sidwell (Michigan State University), N. W. Reay, K. Reibel, N. R. Stanton (Ohio State University), K. W. Edwards, M. Harrison (Carleton University); P. Brindza, H. R. Barton, Jr. (Fermilab)

It is proposed to search for and study two-body decays of narrow, massive, neutral mesons produced near the forward direction in neutron-Be collisions. The apparatus is a high resolution, mass-focusing spectrometer capable of focusing masses up to $-3 \mathrm{GeV} / \mathrm{c}^{2}$ with resolution of $15-30 \mathrm{MeV} / \mathrm{c}^{2}$ FWHM; it consists of a proportional chamber-spark chamber spectrometer constructed around a BM-109 bending magnet. A two-cell 10m long Cerenkov counter allows the identification of decay particles.

The apparatus is sensitive to peripherally or diffractively produced objects and is thus complementary to those that would search in the central region. (For a mass of $2.5 \mathrm{GeV} / \mathrm{c}^{2}$ we detect with good efficiency particles produced with $P_{1} \leq .6 \mathrm{GeV} / \mathrm{c}^{2}$ and $.2 \leq x \leq 1$.) The neutron beam has the advantages that (1) the heavy objects need not be restricted to quantum numbers accessible in $\mathrm{e}^{+} \mathrm{e}^{-}$collisions; (2) beam intensities an order of magnitude higher than those tolerable with charged beams may pass through the detectors.

Summary prepared by

M. ABOLINS, January 1976 


\section{A SEARCH FOR CHARMED PARTICLES}

Harvard University, University of Illinois, University of Oxford, Max Planck Institute, Munich

\section{T. Kirk (Spokesperson)}

The experiment has three basic goals:

i) a search for charmed particle production in strong interactions

ii) mesonic psi production plus measurements on associated hadrons

iii) pion diffraction dissociation at high energy.

In i), we search for peripheral production of $\bar{D} \bar{D}$ containing states using a muon from a semileptonic $D$ decay in coincidence with a kinematically restricted recoil proton. The other $D$ is assumed to decay hadronically and have a fully reconstructable mass some fraction of the time. The $D$ will reveal itself as a narrow ( $50 \mathrm{MeV}$ FWHM) mass peak on a continuum background. We will be sensitive to two and three body decays in which one of the products is identified as a charged or neutral kaon. This trigger is sensitive to peripheral production cross sections of order $0.3 \mu \mathrm{b}$.

In ii), we trigger on 2 opposite sign $\mu^{\prime} s$ in the final state. From this trigger we will acquire a sample of about 800 psi mesons with their accompanying hadrons. We will use these events to look for $\psi n \pi$ resonances and for evidence of associated charmed particles (Zweig Rule). This experiment is dominated by sma11 x (central) production.

In iii) we gather parasitically a sample of 32,000 pion diffraction dissociation events for study of the $A_{1}, A_{2}, A_{3}, A_{4}$ mass region at high energy. This will represent an increase of two orders of magnitude in statistics over present bubble chamber samples and farilitatepartial wave analysis of the results. AIl of the items i), ii), ili) can be obtained simultaneously by using several low rate triggers in logical OR.

We propose to use the CCM spectrometer in the muon laboratory. In addition to the present equipment, we will add a new $\mathrm{LH}_{2}$ target flask and vacuum chamber and two new recoil proton arms.

We request a beam of $\pi^{-}$at $150(\mathrm{GeV} / \mathrm{c})$ and $10^{6}$ per burst. We will need $2 \times 10^{5}$ bursts to complete the experiment as proposed. 
CONTINUED SEARCH FOR NEW PARTICLE PRODUCTION USING ELA DETECTOR

A. Benvenuti, D. Cline, W.T. Ford, R. Imlay, T.Y. Ling, A.K. Mann,

F. Messing, R. Orr, D.D. Reeder, C. Rubbia, R. Stefanski,

L. Sulak and P. Wanderer; Harvard University, University of

Pennsylvania, University of Wisconsin, Fermi National Accelerator Laboratory

During previous running of various types for Experiment \#lA there have been observed several hints of possible new particle production. The indications are as follows:

1) A distortion of the $y$-distribution of antineutrino events at high energy.

2) A possible breakdown of charge symmetry invariance at small $x$.

3) A distortion of the expected distribution of the mass of the recoiling hadrons.

4) The existence of dimuon events at the level of $-5 \times 10^{-3}$ of the total number of events.

In order to pursue this matter further the experimenters have requested a running period with about 1018 protons on target while using the quadrupole triplet train load. A spill time of $1 \mathrm{msec}$ is also requested. 
THE INVESTIGATION OF THE PRODUCTION OF HEAVY FRAGMENTS INDUCED BY PARTICLES AT HIGH ENERGIES

M. Jurić, R. Antanasijević, $\stackrel{Z}{Z}$. Todorović

(Institute of Physics, Belgrade)

Solid state track detectors will be exposed to a high energy hadron beam to investigate the production of heavy nuclear fragments. The cross sections for these reactions will be studied as a function of the energy of the incident particle and of the atomic number of the target nucleus. Particular emphasis will be placed on the fission processes for which a dependence is already observed in the range of 10-25 GeV.

A $3 \times 3 \times 1 \mathrm{~cm}^{3}$ stack of solid state track detectors would be exposed to a beam with a flux of $1011 / \mathrm{cm}^{2}$. The detectors permit the study of fragment emission under very favorable conditions: a low level of background tracks, good spatial resolution, precise measurement of track length and reaction angles. The stack will be analyzed by the Institute of Physics, Belgrade.

Summary prepared by

W.F. Baker, February 1975 
PROPOSAL TO SEARCH FOR LONG LIVED PARTICLES AT FERMILAB

U.J. Becker, J. Burger, M. Chen, R. Forber, J. Leong, T. Rhoades, S.C.C. Ting, and S.L. Wu (Massachusetts Institute of Technology)

This group proposes to use a two armed magnetic spectrometer system to search for two-and three-body decays of long-lived particles. Following their Brookhaven run it would extend the mass range from $5.5 \mathrm{GeV}$ up to $10 \mathrm{GeV}$.

The questions they will attempt to answer are:

1. Are there long-lived particles which have the quantum numbers $0^{+}, 2^{+}, 3^{-}$etc. and which decay into pp, $\mathrm{K}^{+} \mathrm{K}^{-}$, etc., but not to $\mathrm{e}^{+} \mathrm{e}^{-}$?

2. Are there long-lived baryon states $\pi^{-} p, K^{-} p$, etc.?

3. Are there long-lived strange mesons $\mathrm{K}^{+} \pi^{-}, \mathrm{K}^{-} \pi^{+}$, etc.?

4. Are there charged $\mathrm{J}$ particles, such as $\mathrm{J}^{+}+\mathrm{e}^{+} \mathrm{K}^{+} \mathrm{e}^{-}$or $\mathrm{J}^{-}+\mathrm{e}^{-} \mathrm{K}^{-} \mathrm{e}^{+}$, which would show up as a threshold cutoff in mass at $\mathrm{m}_{\mathrm{J}^{+}}$when one detects $\mathrm{e}^{+} \mathrm{K}^{+}$in coincidence?

5. Are there charmed particles which decay to Ke $v$, which will also distinguish themselves with a cutoff behavior at the mass $m_{J}$ when one detects $\mathrm{ke}$ in coincidence?

The two spectrometer arms would look in the region of $90^{\circ}$ in the center-of-mass. Each arm would bend vertically to measure momentum, would have two Cerenkov counters to identify pions and kaons, proportional wire chambers, scintillation counter hodoscopes, two banks of lead glass counters, and one bank of lead lucite shower counters. The angular acceptance would be $\Delta \theta^{*}= \pm 6^{\circ}$ and $\Delta \phi^{*}= \pm 8^{\circ}$. The mass acceptance would be $\Delta \mathrm{M}=3.0 \mathrm{BeV}$, permitting the mass range from 2 to $10 \mathrm{GeV}$ to be covered in three steps. The mass resolution would range from $\pm 4 \mathrm{MeV}$ at $\mathrm{m}_{\mathrm{J}}=2 \mathrm{GeV}$ to $\pm 7 \mathrm{MeV}$ at $\mathrm{m}_{\mathrm{J}}=8 \mathrm{GeV}$

They request 1200 hours of running and 200 hours of beam tuning. This would yield a sensitivity down to $10^{-34} \mathrm{~cm}^{2}$. 


\section{INTERACTION OF 50-100 GeV MUONS WITH EMULSION NUCLEI}

Piyare I. Jain (State Univ. of New York at Buffalo)

Recently we have reported the direct-electron-pair production from the coulomb field of an emulsion nucleus by 200 $\mathrm{GeV}$ protons ${ }^{1}$ and $150 \mathrm{GeV}$ muons ${ }^{2}$ from the Fermi Laboratory and $15.8 \mathrm{GeV} / \mathrm{c}$ muons ${ }^{3}$ from the Brookhaven National Laboratory, in order to test the predictions of the theories based on quantum electrodynamics at small distances. From most of the present theories $4-7$, the predictions for electron production cross section depends on the ratio $\gamma(=E / m)$ and not on $E$ and $m$ separately or on the type of the incoming particie. Our recent results indicates that mass of the incoming particle is perhaps playing an important role. In order to check this point we would like to have two or three more muon beam exposures between 50-100 GeV at FNAL. These experiments will help us to settle the mass dependence question very clearly.

\section{References}

1. P.L. Jain, M. Kazuno, B. Girard, and 2. Ahmad, Phys. Rev. Lett. $32,797(1974)$.

2. P.L. Jain, M. Kazuno, and B. Girard, Phys. Rev. Lett. 32, $1400(1974)$.

3. P.L. Jain, M. Kazuno, and B. Girard, Phys. Rev. (accepted Feb. 1975).

4. H.J. Bhabha, Proc. Cambridge Phil. Soc. 31, 394 (1935) and Procd. Roy. Soc. A, 152, 559 (1935).

5. G. Racah, N.C. 14, $93(1937)$.

6. T. Murota, A. Weda and H. Tanada, Progress Theor. Physics, 16, $482(1956)$.

7. M.M. Block, D.T. King and W.W. Wada, Phys. Rev. 96, 1627 (1954).

Summary prepared by

P.L. Jain, March 1976 
A SEARCH FOR CHARMED PARTICLES ORIGINATING FROM THE INTERACTIONS OF $300 \mathrm{GeV} / \mathrm{C}$ PROTONS IN EMULSION NUCLEI

G. Coremans-Bertrand, J. Sacton (Université Libre de Bruxelles);

T. Cantwell (College of Technology, Dublin); A. Breslin,

G. McMahon, A. Montwill (Univ. College, Dublin); D.R. Davis,

D.N. Tovee (Univ. College, London); F.R. Stannard (Open Univ.);

N. Kurtz, M. Jung, M. Paty (Université de Strasbourg);

G. Baroni, S. Di Liberto, A. Manfredini, S. Petrera, G. Romano (Universita di Roma); T. Pniewski (Universytet Warszawski)

The exposure of the emulsion stack was made in June 1975 with the object of locating possible examples of the associated production of charmed particles by high energy proton collisions. The high spatial resolution of the nuclear emulsion technique, a few microns, should enable the direct observation of the decays of such particles with lifetimes as small as $10^{-14} \mathrm{~s}$. To date no possible examples of pairs of charmed particles decays have been seen within a $45^{\circ}$ forward cone extending some $150 \mu \mathrm{m}$ from the primary interaction vertex for a sample of 30,000 proton interactions. This preliminary result implies, provided that charmed particle lifetimes are in the range $10^{-14}$ to $10^{-12} \mathrm{~s}$, an upper limit of $\sim 1 \mu$ for their production by $300 \mathrm{GeV} / \mathrm{c}$ proton interactions. The scanning of the emulsions is continuing.

Summary preparea by

D. H. Davis, March 1976 


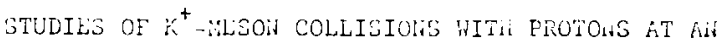

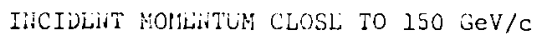

L. Dunh, H. lieller, A.r. Shapiro, A. Widgoff (brown Univ.); F. Druyant, Y. Goldschridt-Clemont, L. Montanet, A. Stegiou (CLRii); R.A. Burnstein, C. Fu, H. Vazaretz, li.A. Rubin (IIT); J. Brau. J. Grunhaus, L. Hafen, R.I. Hulsizer, U. Karshon, V. Kistiakowsky, A. hapier, I.A. Fless, J. Holfson R.K. Yamanoto (iII); W. Kittel, K. Hetzger, C. Yols, J. Schotanus, K. Van de Walle (ifijmegen Univ.); it. Braun, A. Fridman, J.P. Gerber, P. Juillot, G. Vaurer, A. Michalon M.E- Michalon-Menter, C. Voltolini (Strashourg). b. Logert, M. Johnson,

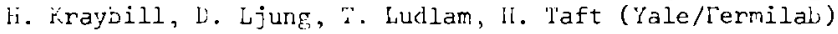

It is proposed to make exposures of approximately $0.5 \mathrm{ev} / \mu \mathrm{L}$ equivalent for $\mathrm{K}^{+}$ Inesons as close as possible to $150 \mathrm{GeV} / \mathrm{c}$ in the Fermilab 30 -inch bubble chamber proportional wire hybrid systen. These exposures are requested as soon as suitably enriched beams of $\mathrm{k}^{+}$mesons are available to that facility. For beams with $\mathrm{k}^{+} / \mathrm{con}^{-}$ taminant ratio, $P$, the exposure will require $1 / R \times 100,000$ pictures anci will yield approximately $10.000 \mathrm{~K}^{+} \mathrm{p}$ interactions for study.

The purpose of these experiments is to carry out a detailed study, with modest statistical accuracy, of kan proton collisions at energies significantly above those at wich such data currently exists, and to furtner extend the range of incicient channel quantum numbers over which multiparticle production has been explored at Fermilai energies. The choice of cnergy is such as to allow us to make a reasonable cross particle comparison with existing data at $147 \mathrm{GeV} / \mathrm{c}$. Some of the specific topics to be studied are toinological cross sections, elastic cross sections, leading particle effects at low-t, resonance prociuction, central emission, and douvle diffraction dissociation.

An interral part of this proposal is the development of the $\mathrm{h}^{+}$meson bean. During tile sumner of 1975 the experimenters nacie a study of a scinerie for producing an enriched $\mathrm{h}^{+}$Leam, and have proposed the installation of a polyetiylene filter in the ii beam line to produce a beam with $155<R<20 \%$. It is expected that this proposal will proceed in two phases. The first phase will be a further feasibility study of the $\mathrm{r}^{+}$beam and the second phase, contingent on the success of the first phase, will be the proposed physics exposure. 
STUDIES OF $\mathrm{K}^{-}$-MESON COLLISIONS WITH PROTONS AT AN INCIDENT MOMENTUM CLOSE TO $150 \mathrm{GeV} / \mathrm{C}$

D. G. Fong, M. Heller, A. M. Shapiro, M. Widgoff (Brown Univ.);

F. Bruyant, Y. Goldschmidt-Clermont, L. Montanet, A. Stegiou

(CERN); R. A. Brunstein, C. Fu, D. V. Peterson, R. M. Robertson, H. A. Rubin (IIT); J. Grunhaus, E. Hafen, R. I. Hulsizer,

U. Karshon, V. Kistiakowsky, A. Napier, I. A. Pless,

P. Trepagnier, J. Wolfson, R. K. Yamamoto (MIT); W. Kittel,

W. Metzger, C. Pols, J. Schotanus, R. Van de walle (Nijmegen

Univ.); Y. Eisenberg, B. Haber, D. Hochman, G. Mikenberg,

E. E. Ronat, A. Shapira, R. Yaari, G. Yekutieli (Weizmann Inst.);

D. Bogert, M. Johnson, H. Kraybill, D. Ljung, T. Ludlam,

H. Taft (Yale/Fermilab)

It is proposed to make exposures of approximately 0.5 ev/ $\mu \mathrm{b}$ equivalent for $\mathrm{K}^{-}$mesons as close as possible to $150 \mathrm{GeV} / \mathrm{c}$ in the Fermilab 30-inch bubble chamber proportional wire hybrid system. We request these exposures as soon as suitably enriched beams of $\mathrm{K}^{-}$mesons are available to that facility. For beams with $\mathrm{K}^{-}$/contaminant ratio $\mathrm{R}$, each exposure will require $1 / R \times 100,000$ pictures and will yield approximately $10,000 \mathrm{Kp}$ interactions for study.

The purpose of these experiments is to carry out a detailed study, with modest statistical accuracy, of kaonproton collisions at energies significantly above those at which such data currently exists, and to further extend the range of incident channel quantum numbers over which multiparticle production has been explored at Fermilab energies. The choice of energy is such as to allow us to make a reasonable cross particle comparison with existing data at $147 \mathrm{GeV} / \mathrm{c}$. Some of the specific topics to be studied are topological cross sections, elastic cross sections, leading particle effects at low-t, resonance production, central emission, and double diffraction dissociation.

A fundamental part of this proposal is the development of the $\mathrm{K}^{-}$meson beam. The experimenters are prepared to explore the particle enrichment schemes being studied for the hadron beams to the bubble chambers, either alone or in collaboration with other interested parties. We are also prepared to study alternate $\mathrm{K}^{-}$beam producing techniques. It is expected that this proposal will proceed in two phases. The first phase will be a feasibility study of the $\mathrm{K}^{-}$beam and the second phase, contingent on the success of the first phase, will be the proposed physics exposure.

Summary prepared by

T. W. Lualam/H. A. Rubin, January 1976 


\section{A PRECISION STUDY OF LEADING PARTICLE AND DIFFRACTIVE EFFECTS IN $\pi{ }^{-} \mathrm{p}$ INTERACTIONS AT $300 \mathrm{GeV} / \mathrm{C}$}

M. Heller, A. M. Shapiro, M. Widgoff (Brown Univ.); F. Bruyant, Y. GoldschmidtClermont, L. Montanet, A. Stergiou (CERN); J. Grunhaus, E. Hafen, R. I. Hulsizer, U. Karshon, V. Kistiakowsky, A. Napier, I. A. Pless, J. Wolfson, R. K. Yamamoto (MIT); G. Alexander, I. Bar-Nir, O. Benary, S. Dagan, A. Levy (Tel-Aviv);

Y. Eisenberg, B. Haber, D. Hochman, G. Mikenberg, E. E. Ronat, A. Shapira, R. Yaari, G. Yekutieli (Weizmann Institute); H. Kraybill, D. Ljung, T. Ludlam, H. Taft (Yale).

A 200,000 picture exposure is proposed for the Fermilab 30-inch proportional wire chamber hybrid bubble chamber system, including the Forward Gamma Detector, exposed to a $300 \mathrm{GeV} / \mathrm{c} \pi^{-}$beam. It is expected to use the high precision of this system to make a significant contribution to the following topics:

1. Leading particle effects and diffraction phenomena.

2. Charged particle multiplicity moments.

3. Production spectra for charged and neutral particles.

4. Correlations in momentum and rapidity among the produced particles.

j. Total cross section for $\mathrm{K}^{-}$interactions.

6. Semi-inclusive distributions for interactions with an associated forward $\pi^{0}$.

7. Inclusive cross-sections for $\rho^{0}$, forward $\rho^{+}, \rho^{-}$.

8. Charged clusters.

9. Constrained fits to the $\mathrm{p}^{+} \pi^{-} \pi^{-}$final state.

This exposure is expected to yield about 40,000 interactions for our study. The rough breakdown by prong topology (based on a linear extrapolation from previous 30 -inch exposures at $147 \mathrm{GeV} / \mathrm{c}$ and $205 \mathrm{GeV} / \mathrm{c}$ ) is:

\begin{tabular}{|c|c|c|c|}
\hline Type & Number of Events & $\begin{array}{l}\text { Events with } \\
\text { leading p }\end{array}$ & $\begin{array}{l}\text { Events with } \\
\text { leading } \pi\end{array}$ \\
\hline Total & 40,000 & 2,400 & 2,400 \\
\hline 2 prong elastic & 5,000 & & \\
\hline 2 prong inelastic & 2,000 & 800 & 800 \\
\hline 4 prong & 4,000 & 1,000 & 1,000 \\
\hline 6 prong & 4,000 & 400 & 400 \\
\hline 8 prong & 7,000 & & \\
\hline 10 prong & 7,000 & & \\
\hline 12 prong & 5,000 & & \\
\hline 14 prong & 4,000 & & \\
\hline 16 prong & 1,000 & & \\
\hline$\geqq 18$ prong & 1,000 & & \\
\hline
\end{tabular}

In addition, it is expected to use the Cerenkov tagging system to detect and measure about $800 \mathrm{~K}^{-} \mathrm{p}$ and $300 \overline{\mathrm{p} p}$ interactions, assuming beam contaminations of $2 \%$ and $0.6 \%$, respectively.

Data taking should require about 2 weeks. 


\section{A PROPOSAL TO STUDY LEADING PARTICLE PRODUCTION IN $\pi^{-} \mathrm{p}$ INTERACTIONS AT $75 \mathrm{GeV} / \mathrm{c}$}

D. G. Fong, M. Heller, A. M. Shapiro, M. Widgoff (Brown Univ.);

F. Bruyant, Y. Goldschmidt-Clermont, L. Montanet, A. Stegiou

(CERN); J. Grunhaus, E. Hafen, R. I. Hulsizer, U. Karshon,

V. Kistiakowsky, A. Napier, I. A. Pless, P. Trepagnier,

J. Wolfson, R. K. Yamamoto (MIT); W. Kittel, W. Metzger,

C. Pols, J. Schotamus, R. Van de walle (Nijmegen Univ.);

A. Levy, D. Lissauer, Y. Oren, I. Bar-Nir, I. Stumer (Tel-Aviv);

D. Bogert, M. Johnson, H. Kraybill, D. Ljung, T. Ludlam,

H. Taft (Yale/Fermilab)

It is proposed to investigate leading particle effects in the $\pi^{-} \mathrm{p}$ interactions at $75 \mathrm{GeV} / \mathrm{c}$. For this purpose a 200,000 frame exposure is requested to be made with the proportional wire hybrid system (PWHS). The results thus far obtained by the PWHS consortium in a study of $\pi^{-} p$ interactions at $147 \mathrm{GeV} / \mathrm{c}$ demonstrate the ability of the system to measure pion momenta at $75 \mathrm{GeV} / \mathrm{c}$ with the accuracy to resolve the leading particle peak near $x=1$. For a given $x$ of the leading particle, either proton or $\pi^{-}$, measurements can be made of:

1. $d^{3} \sigma / d p$ for the other charged particles produced,

2. multiplicity of other charged particles,

3. multiplicity of other charged particles as a function of recoiling invariant mass.

4. invariant masses of subsets of other particles.

They will investigate to what extent quasi-two body processes occur at this momentum.

In addition to a study of these topics at $75 \mathrm{GeV} / \mathrm{c}$, the proposed experiment, in conjunction with the earlier experiment at $147 \mathrm{GeV} / \mathrm{c}$ and another proposed experiment at $300 \mathrm{GeV} / \mathrm{c}$, will contribute to an understanding of the energy dependence of the leading particle production mechanism. In particular, these experiments will provide a test of the assumption that the leading particle peak is independent of energy. The $s$-dependence of related effects can also be studied in the framework of specific models, such as the double-Pomeron exchange contribution, or the various contributions of the Pomeron and Reggeon couplings within the Triple-Regge Limit Model. 


\section{SEARCH FOR SHORT LIVED STATES DECAYING WEAKLY VIA LEPTONIC MODES}

B. Barish, F. Bartlett, A. Bodek, K. Brown, D. Buchholz,

F. Merritt, F. Sciulli, L. Stutte (Caltech); P. Dishaw,

M. Faessler, A. Hall, D. Hitlin, J. Kirkby, J. Liu,

R. Piccioni, S. Wiesner, S. Wojcicki (Stanford University

This search is for high mass, short lived states decaying weakly via leptonic or semi-leptonic modes. A hadron beam interacts in a fine grain calorimeter permitting the detection of missing energy presumably carried off by neutrinos. There is a large solid angle acceptance for muons which are analyzed in a toroidal spectrometer. The experiment would detect charmed hadrons, color states, heavy leptons and $W$ bosons up to a mass of $20 \mathrm{GeV}$. It would also be sensitive to production phenomena involving single muons and direct neutrinos.

Proportional chambers in the beam line would determine the position of the incident particle and measure its momentum to a precision of 1 or $2 \%$. The calorimeter would be of three parts of increasing size with a total length of 20 absorption lengths. About $70^{\circ}$ of the total energy would be deposited in the first section and the most precise measurements would be made here. The density at the front end would be variable to calibrate the number of pion and kaon decays. The second section would be a scaled up version of the first; wide angle components would be identified in it. The last section would be coarse grained and in it would be analyzed the anomalously long hadron showers. The calorimeter resolution should be about $\pm 4 \%$ at $400 \mathrm{GeV}$. The muon spectrometer consists of two magnetized toroids 11.5 feet in diameter followed by one 5 foot diameter toroid. Wire chambers are placed on both sides of each toroid. Momentum resolution of $\pm 9 \%$ is expected for any two toroids. The overall energy resolution of the system would be $\pm 20 \mathrm{GeV}$.

Three types of triggers would be used. The simplest, the energy loss trigger, would require a significant deposition of energy in the calorimeter, indicating the production of muons or neutrinos. The single muon trigger would require at least one muon with some minimum transverse momentum. At lower transverse momenta a di-muon trigger would be used to study multi-muon states. With no low-pt cutoff, the raw trigger rate would be 30-50 per pulse; with such a cutoff, this rate would be reduced an order of magnitude. An estimate indicates that a background of some tens of events per day is to be expected. A run of 1000 hours is requested in a hadron beam of $10^{6}$ protons/pulse at the highest energy possible. This would yield a sensitivity of $10^{-36}-10^{-37} \mathrm{~cm}^{2}$. 
STUDY OF THE PROPERTIES OF WEAK NEUTRAL CURRENTS IN THE INTERACTIONS OF A NARROW BAND NEUTRINO BEAM IN LIQUID NEON

C. Baltay, M. Kalelkar (Columbia); R. B. Palmer,

N. P. Samios (BNL)

Since it has already been established that neutral currents exist, the next step is to study their detailed properties. For this experiment the main features will be a) knowledge of the incident neutrino energy and direction, and $b)$ detection and identification of the individual hadrons produced through study of their behavior in a liquid neon fill of the 15-foot bubble chamber. The primary physics questions to be answered are:

1) What is the primary structure of the neutral currents, $(V, A)$ or $(S, P)$ ? This should be answered through the shape of the $y$ distribution (where $y=E$ outgoing hadron/E incident neutrino).

2) What is the total cross section for the neutral current events for several values of the incident neutrino energy? It will be possible to make more reliable cross section measurements when the $y$-distributions are better known.

3) What are the structure functions for inelastic neutral current interactions? These can be crudely determined from the $\mathrm{x}$ - and $\mathrm{y}$-distributions.

4) What is the nature of the specific hadrons produced in the neutral current events? These may be determined from the behavior of the secondary particles in the chamber.

The proponents of this proposal have designed a narrow band beam to use two pulsed magnetic horns. This would primarily involve construction of new inner conductors for the existing horn focusing wide-band train load. A momentum bite of $+5 \%$ is expected with an integrated neutrino flux comparable to those from the train loads mentioned above. In a 200,000 picture exposure, with a 24 metric ton neon-hydrogen fill of the 15-foot chamber, with an average of $5 \times 1012$ protons/ pulse on target at $300 \mathrm{GeV}$, and the existing narrow band trainload, one would expect about 5,200 charged current and 1,300 neutral current events. A new beam would provide an improved momentum spread and a larger number of events.

Summary prepared by A. F. Greene, March 1975

Modified by

C. Baltay, January 1976 
MEASUREMENT OF THE REAL PART OF THE $\mathrm{p}-\mathrm{n}$ and $\mathrm{p}-\mathrm{p}$ FORWARD SCATTERING AMPLITUDES; PRODUCTION OF LOW MASS ISOBARS IN THE VERY SMALL MOMENTUM TRANSFER REGION

University of Arizona, Joint Institute of Nuclear Research, USSR, Fermi National Accelerator Laboratory, University of Rochester, Rockefeller University

E. Malamud (Spokesperson) goals:

An internal target experiment is proposed with the following

1. A measurement of the real part of the p-d forward scattering amplitude from $8 \mathrm{GeV}$ to $\geq 400 \mathrm{GeV}$. the $\mathrm{p}-\mathrm{n}$ real part would be extracted from the data using Glauber theory and compared with measurements of the $\mathrm{p}-\mathrm{p}$ real part. The highest energy $\mathrm{p}-\mathrm{n}$ real part measurements to date are $70 \mathrm{GeV}$.

2. A remeasurement of the real part of the $p-p$ forward scattering amplitude from $8 \mathrm{GeV}$ to $400 \mathrm{GeV}$ using the same apparatus as the $p$-d measurements to facilitate the $p-p, p-n$ comparison. By using a long ion guide and position sensitive detectors the accuracy attainable will be significantly improved over previous measurements in Experiment \#36A.

3. Measurements of the production of low mass isobars $1.0<M_{x}<2.0 \mathrm{GeV} / \mathrm{c}^{2}$ in the very small momentum transfer region $.005<|t|<.025(\mathrm{GeV} / \mathrm{c})^{2}$. The use of a 7.5 meter, long ion guide and position sensitive detectors will result in a mass resolution of $16 \mathrm{MeV}$, a significant improvement over previous measurements in Experiments \#186 and \#317.

No additional equipment is required beyond what is already proposed and partly under construction for Experiment \#289 by the same participants involving proton-helium scattering. The performance of these proposed measurements with hydrogen and deuterium is a logical first step in commissioning the apparatus for Experiment \#289. The proposed measurements would require approximately 300 hours. There is a need for one week of data up to $400 \mathrm{GeV}$, and interest in collecting data above $400 \mathrm{GeV}$. 
A SEARCH FOR CHARMED HADRONS PRODUCED BY MUON DEEP INELASTIC SCATTERING IN TAGGED NUCLEAR EMULSIONS

K. Gottfried, L. Hand, S. Herb (Cornell Univ.); L. Read and

L. Voyvodic (Fermilab); L. Litt (Michigan State Univ.);

J. Lord, J. Florian, J. Wilkes (Univ. of washington)

A relatively simple and efficient method for searching for charmed hadrons is proposed. The experiment has three basic ingredients: 1) deep inelastic muon scattering which is conjectured to be a rather copious source of charmed particles, 2) an emulsion stack to explicitly display the decay vertex, and 3) very precise triangulation with drift chambers to locate the event and greatly reduce scanning time. The inelastically scattered muons would be identified with the muon spectrometer from Experiment \#26; the drift chamber system is similar to one already in operation at the cornell synchrotron. The goal is to obtain 500-1000 emulsion stars known to have been produced by deep inelastic muon scattering. The experimenters have reason to believe that 10-20\% of these may contain charmed hadrons, or other new phenomena. An inevitable byproduct of this experiment would be the first determination of how multiple production in a heavy target depends on $\mathrm{q}^{2}$. A secona important byproduct would be a search for "extra" muons in deep inelastic scattering.

The work needed to complete the exposure includes reactivation of the spectrometer from experiment \#26, a prototype exposure and the main exposure consisting of about $10^{8}$ incident muons ( 100 pulses).

Summary prepared by

L. Hand, February 1975 
A PROPOSAL TO STUDY THE INCLUSIVE PRODUCTION OF $\mathrm{K}_{\mathrm{S}} \mathrm{BY} \mathrm{K}$ ON HYDROGEN

H.G.E. Kobrak, R.E. Pitt, R.A. Swanson (University of California, San Diego)

2 It is proposed to measure the inclusive cross section $\mathrm{d}^{2} \sigma / \mathrm{dxd}$ for the reaction $\left(K^{-} \mathrm{P}\right) \rightarrow\left(K \mathrm{~K}_{S} \mathrm{X}\right)$ with incident kaons of 20 to $150 \mathrm{GeV} / \mathrm{c}$. The experiment is intended to study the triple Regge region of the $x, t$ plane $(0.8<x<1,0<-t<3)$. In this region results will be compared with the triple Regge theory. This process should involve only $\rho$ and $A_{2}$ exchange, which implies a relatively simple situation for analysis. By adding the constraints of $\mathrm{SU}_{3}$ and factorization, one can make direct comparison with the production of $\pi^{\circ}$ and $n$ by $\pi^{-}$.

The experiment would be suited for the proposed charged mode of the $M 4$ beam line. The $K \&$ would be detected by the $\left(\pi^{+}, \pi^{-}\right)$decay in a pair spectrometer such as that used in the $\mathrm{Kg}$ regeneration experiment $\mathrm{E}-82$. Downstream from the $30 \mathrm{~cm}$. hydrogen target is a vacuum drift space whose length is adjusted to maximize the detection efficiency and minimize the incident beam interference. Then follows a standard wire chamber spectrometer with two $10 \mathrm{~m}$. legs bracketing the M4B8 analysis magnet. Sweeping magnets before and after the target will remove all charged particles from the detection apparatus.

The experimenters expect to participate in the installation and tuneup of the new charged beam. This time is difficult to estimate because of access problems. Once the beam is ready, they request two separate running periods. 200 hours for setup and initial data taking, followed by a period of data analysis before a final run of 300 hours. Typical event rates would be $2000 / \mathrm{hr}$ at $100 \mathrm{GeV} / \mathrm{c}$ for $0<-t<1$ and 17 for $2<-t<3$.

Summary prepared by

W.F. Baker, March 1975 


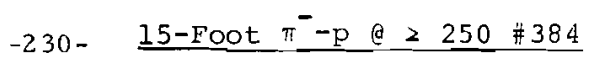

PROPOSAL TO STUDY $\pi^{-}$p INTERACTIONS AT HIGHEST ENERGY IN THE FEPMILAB 15' BUBBLE CHAMBER

D. Bogert, R. Hanft, R. Harris, F.R. Huson, S. Kahn, W. Smart (Fermilab); J.R. Albright, S. Hagopian, V. Hagopian, P. Hays, J. Lannutti (Florida State Univ); C.Y. Chang, R. Glasser, D.G. Fill, M. Kazuno, G. Snow (Univ. of Maryland)

This is a proposal to study hadronic physics with 300,000 pictures in the Fermilab 15-foot bubble chamber filled with hydrogen and exposed to a $\pi^{-}$beam at the highest practicable energy $(\geq 250 \mathrm{GeV})$. The objectives of the experiment are (i) to search for charm particles, (ii) to investigate cluster formation in events containing more than one strange particle, (iii) to study inclusive production of strange particles, (iv) to study inclusive processes involving photons, and (v) to measure parameters of diffraction dissociation.

Negative pions are chosen because they allow the study of direct meson interactions and secondly because the identification of particles in the final state is easier since the beam does not introduce strangeness or baryon number. The highest energy is desired so as to allow for the highest mass of products.

A total of 300,000 pictures are requested in the $15^{\prime}$ hydrogen filled chamber. Assuming 4 beam tracks per picture, and that the $\pi^{-}$p cross section at $35 \mathrm{GeV} / \mathrm{c}$ is $\sim 24 \mathrm{mb}$, they expect a total of $\sim 200,000$ primary interactions yielding an overall rate of 8 events per $\mu b$.

The film will be analyzed beginning with a first pass scan for double $V$ events, using the effective mass of the two V's as a "trigger" to reduce the amount of measurement and will produce preliminary physics results on the first 3 subjects mentioned above in about six months from the conclusion of the run. The second pass scan will concentrate on finding four-prongs and six-prongs, measuring them on an automatic device and getting preliminary physics results in about a year. The full extent of the experiment will take 2-3 years. 
STUDY OF $400 \mathrm{GeV} / \mathrm{C}$ PROTON-NUCLEUS INTERACTIONS

IN NUCLEAR EMULSION

I.S. Mittra, J.M. Kohli, P.M. Sood, B.K. Agrawal, J. Singh (Punjab Univ.); B. Bhowmik, S. Singh, P. Sen Gupta (Univ. cf Delhi); S. Lokanathan, S. Bhalla (Rajasthan Univ.); N.K. Rao, G.L. Kaul, V.K. Gupta, Gyan Singh, S.K. Sharma, S.K. Badyal, Balvinder Kour, Y. Prakash (Jammu University)

Studies of proton-nucleus interactions up to $200 \mathrm{GeV} / \mathrm{c}$ indicate that no single model of multiparticle production seem to explain all the experimentally observed features. Hence, for the experimental data on the multiplicity of charged particles, angular distribution of charged secondaries, rapidity distribution of shower particles, particle correlations and fluctuations etc. are necessary. The present experiment is aimed towards studying these problems.

Recently there have been a revival of the interest in that the fire ball mechanism of high energy interactions. The experimental data will also be used for the study of this problem.

It is assumed that the transverse momentum $\left(p_{t}\right)$ is practically constant in such interactions. It is proposed that $p_{t}$ measurements will be made on part of the data.

In the first phase, measurement of the multiplicity of charge particles, angular distribution of secondaries and rapidity of shower particles is being studied. At present various models for production of charged particle secondaries in hadron-nucleus collisions are in vogue; e.g. Statistical model, Gottfried's energy flux model, multi-pe-ipheral interactions including KNO or Feynmann scaling etc. The data will help in checking the precictions of these models and in understanding the behavior of such interactions as compared to hadron-hadron interactions.

Recently, a lot of interest has been aroused in the search of charm particles. It is likely that such particles may be produced in such interactions which are termed as "Core-core" interactions. Such interactions may also be high pt events. Efforts are being made to study such events and look for the existence of 'charm' like particles.

The technique used for these studies is Nuclear Emulsion.

Summary prepared by

Y. Prakash, April 1976 
A SEARCH FOR LOW ENERGY NEUTRAL PARTICLES AND PARTICLE INTERACTIONS INVOLVING SMALL ENERGY EXCHANGES IN THE NEUTRINO BEAM

Jere J. Lord, Richard J. Wilkes (University of Washington)

A proposal is presented to search for any new type of low energy neutral particles capable of passing through the large neutrino shield and producing nuclear interactions in s stack of nuclear emulsions. The experiment exploits the capability of the nuclear emulsion to detect interactions involving energy exchanges of the order of several MeV which could not be detected with other instruments.

We would like to get a site near the end of the neutrino shield and in line with the beam where a search could be made for new particles of low energy or processes involving small energy exchanges. This experiment obviously is not intended to compete with the large scale neutrino experiments in progress but is aimed at the search for several MeV interactions which could not be detected by conventional means. In addition, the experiment could be carried out with negligible interference with other experiments.

A crude shelter would be needed near the end of the neutrino shelter in order to keep the emulsions out of the weather as well as to provide an area where the emulsions could be coated. Shielding would be required to the extent of providing a box of about 1 ft $^{3}$ surrounded by a shield of several feet of iron or concrete. We would try to keep emulsions in the beam for all periods when large beams are striking the target.

Summary prepared by

J. Lord, J. Lach May 1976 
J. R. Florian, M. Y. Lee, J. J. Lord and R. J. Wilkes (University of Washington)

Target layers consisting of tungsten, silver, chromium, and silver bromide powders (typical target granule diameter $\leqslant 20 \mu$ ) were embedded in $600 \mu$ thick nuclear emulsion plates, and exposed to a $200 \mathrm{GeV} \pi^{-}$beam at ENAL in June, 1975. The plates are currently being scanned for multiparticle production events. For each event found, the multiplicities $n_{s}$ (number of minimumionizing secondaries) and $\mathrm{N}_{\mathrm{h}}$ (number of heavily-ionizing secondaries) are determined, and the angular distribution of minimum-ionizing particles is measured. The experiment is expected to yield valuable information on the $A$-dependence and, by comparison with the results of earlier proton-beam exposures (E-17I, E-237), projectile-dependence of hadron-nucleus multiparticle production processes.

Summary prepared by

R. J. Wilkes, January 1976 


\section{$-234-$ \\ $15-$ Foot $\nu \& \bar{\nu} / \mathrm{H}_{2} \& \mathrm{Ne} \# 388$}

PROPOSAL TO STUDY NEUTRAL CURRENT NEUTRINO AND ANTI-NEUTRINO INTERACTIONS IN THE 15-FOOT BUBBLE CHAMBER USING THE EXTERNAL MUON IDENTIFIER AND A DICHROMATIC BEAM

University of Hawaii, LBL, Fermilab M.L. Stevenson (Spokesperson)

It is proposed to use dichromatic neutrino (antineutrino) beams to study neutral currents (NC), possible breakdown in scaling of charged currents (CC), evidence for lepton pairs and their suggestion of charm-particle production, and other phenomena that require "one-constraint" analysis. Backgrounds and systematic errors would be smaller for these events and it is hoped to use them to reexamine the "zero-constraint" analysis of the broad band beam events that will have been analyzed in earlier experiments, thereby, reducing the systematic errors of those previous analyses. Ne- $\mathrm{H}_{2}$ mixtures (e.g. $1 / 3 \rho$ Ne to $2 / 3 \rho \mathrm{Ne}$ ) in the 15-foot bubble chamber are necessary in order to determine the hadronic final state four-momentum. An exposure of 200,000 $(200,000)$ neutrino (antineutrino) pictures using a monohorn of the type that accepts $100 \pm 5 \mathrm{GeV}$ mesons between 2 and 8 milliradians, produced by $300 \mathrm{GeV}$ protons (1013 per pulse) produces the following number of events:

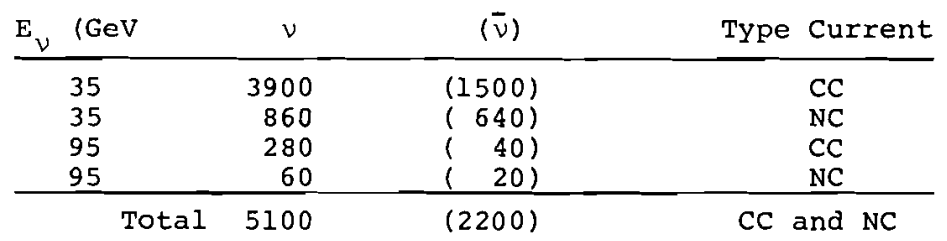

There is a possible need to run the antineutrinos at a higher density, $\rho \simeq 2 / 3 \rho \mathrm{Ne}$, because of event rate and other reasons. The use of other types of dichromatic beams is also considered.

When studying neutral currents the Weinberg-Salam gauge symmetry model can be tested. In the search for scale breakdown the possible existence of a low mass neutral intermediate boson will be examined. Such a particle would distort the $x, y$ distribution, where $x=Q^{2} /(2 M \nu)$ and $y=\left(E_{\nu}-E_{\mu}\right) / E_{\nu}$, and reduce the number of events above $Q^{2} \simeq M_{W}$. A different form of scale breakdown would be caused by the production of new massive hadrons in neutral current events. Here, one expects an increase of neutral current events at high $y$ and low to medium values of $x$.

The $\mathrm{x}$ and $\mathrm{y}$ distributions will also be examined for charged current events. Total cross sections would be more reliably measured with dichromatic beams. Events in exclusive channels would be analyzed as $1-C$ events in the dichromatic beams. Some 40 or 80 dilepton events would be detected, depending on the $\mathrm{Ne}-\mathrm{H}_{2}$ mixture. The external muon identifier would be used in this study. 
PROPOSAI FOR STUDYING NEUTRINO INTERACTIONS IN NEON WITH A NARROW BAND NEUTRINO BEAM IN THE 15' BUBBLE CHAMBER

University of Amsterdam, Universita di Bologna, CEN-Saclay, Universita di Torino A. G. Tenner (Spokesperson)

An experiment is proposed to study neutrino interactions in the $15^{\prime}$ bubble chamber filled with a heavy neon-hydrogen mixture using a narrow band neutrino beam. The main purpose of the experiment is the detailed study of the weak neutral current interactions and the cross section measurement of specific reaction channels.

A comparison with cross section measurements at other energies would enable one to see if the ratio of neutral to charged current events is constant or depends on energy. Knowing the incident energy one can make truly inclusive measurements, particularly of the form: $\quad N N \rightarrow+$ hadrons. A study of the $Y$ distribution of each event is sensitive to the nature $(V, A)$ or $(S, P)$ of the neutral current. Combined $x$ and $y$ distributions are dependent on the structure functions for neutral current events. A comparison would be made of inclusive neutral current events and inclusive charged current events. An additional study would be made to see if individual inelastic channels scale separately. Although the statistical accuracy obtainable in this exposure is admittedly not high, a search would be made for any new phenomena.

With $2 \times 10^{13}$ protons per pulse at $300 \mathrm{GeV}, 1$ event every 10 pictures is expected. A total of 100,000 pictures (or 10,000 neutrino events) is requested.

It is assumed that the chamber fill is from $80 \%$ to 908 neon and that a $30 \mathrm{kG}$ field is available. Possible beams are the existing narrow band beam of E-2l, the proposed dipolequadrupole narrow band beam designed by E2I people, and the proposed two-horn narrow band beam. Any of these would be run at $90-120 \mathrm{GeV} / \mathrm{c}$ if the accelerator is at $300 \mathrm{GeV}$. The experimenters would like to use the external muon identifier.

As a feasibility and rate study the experimenters would like to have an early run of 5,000 pictures with the existing narrow band beam and whatever neon-hydrogen bubble chamber filling is available.

Summary prepared by

W. F. Baker, May 1975 


\section{ANTINEUTRINO INTERACTIONS IN THE DEUTERIUM FILLED 15-FOOT BUBBLE CHAMBER}

Purdue University, Carnegie-Mellon University and Argonne National Laboratory A.F. Garfinkel (Spokesperson)

It is proposed to study the interactions of antineutrinos in deuterium. The initial request is for 300,000 pictures out of a total of one million pictures using the two-horn broad band system with $10^{13}$ protons $(300 \mathrm{GeV})$ per pulse. We plan to use the external muon identifier.

The physics motivation includes the following:

1. Search for charmed mesons.

2. Study of elastic and inelastic hyperon production. Only in antineutrino interactions can we study the $\Delta S=1$ charged current in quasi two-body interactions at energies and momentum transfers higher than that available in decays.

3. Study of the quasi elastic and pion production reactions. These data will be combined with the vp data of our experiment $(E-31)$.

4. Inclusive study of charged current interactions off neutrons and protons.

5. We will study the structure of the neutral current interaction. We will check the absence of $\Delta \mathbf{S}=1$ neutral currents.

6. We will also look for di-muon events using the EMI.

7. We are interested in the possibility of inserting a threeradiation length metal plate in the bubble chamber to serve as a gamma converter and an electron detector.

Summary prepared by

A. F. Garfinkel, April 1975 


\section{EXPLORATION OF RARE MUON-INDUCED PROCESSES}

A.R. Clark, E.S. Groves, L.T. Kerth, S.C. Loken, M. Strovink. W.A. Wenzel (U.C. Berkeley - LBL); R. Cester, F.C. Shoemaker, P. Surko, M.S. Witherell (Princeton); R.P. Johnson (Fermilab)

Initial Physics Objectives will be pursued concurrently:

1. Search for heavy neutral muons predicted by gauge theories in the mass range 2 - $10 \mathrm{GeV}$, and other new particles decaying into one muon. The sensitivity for analyzed events is $1-2 \times 10^{9}$ events/ $\mu b$.

2. Measurement of deep-inelastic virtual Compton scattering and search for other deep-inelastic $\mu^{+} \mu^{-}$pair creation.

3. Measurement of the deep-inelastic muon scattering structure function at very high momentum transfer. For example, 8200 events exceeding $Q^{2}=160(\mathrm{GeV} / \mathrm{c})^{2}$ will be obtained.

4. Collection of $2-3 \times 10^{4} \psi+\mu^{+} \mu^{-}$decays and data at higher dimuon masses. The $\psi \mu \mu$ coupling will be measured in the range $-15(\mathrm{GeV} / \mathrm{c})^{2}<\mathrm{q}^{2}<0$ with 78 statistics at $\mathrm{q}^{2}=-\mathrm{m}_{\psi}^{2}$.

Experimental Technique

The spectrometer will realize a broader range of muon physics objectives than those accessible to the first-round muon scattering experiments. It consists of 18 modules, each containing 5 magnetized iron plates, 5 calorimeter counters, 1 proportional chamber, and 1 drift chamber. Alternate modules contain 1 trigger hodoscope. The necessary sensitivity to rare processes is provided by high acceptance over the full length of the distributed target-spectrometer. Nearly continuous iron is used to suppress $\pi \rightarrow \mu \nu$ decay background and provide the medium for calorimetric determination of energy transfer to hadrons. Fast detectors will tolerate further increases in beam intensity while maintaining acceptance of multiple final-state muons even within the beam area. The various background trigger rates have been estimated and found to be manageable.

Requirements for Operation

Existing space in the north portion of the Muon Laboratory is required, with an illumination of 1012 muons at the highest available energy $(225 \mathrm{GeV}$ has been assumed). The experiment is compatible with normal operation of the Muon scattering Facility upstream.

Summary prepared by

M. Strovink, February 1975

Note: The above summary describes the objectives of both Proposals \#203A and \#391. The specific physics objectives of Proposal \#391 are expressed in items 3. and 4. above. (AFG) 
PROPOSAL TO STUDY MULTIPARTICLE PRODUCTION AT THE HIGHEST AVAI LABLE MOMENTUM IN ANTIPROTON-PROTON INTERACTIONS USING THE FERMI LAB 30-INCH BUBBLE CHAMBER

R.E. Ansorge, J.R. Carter, W.W. Neale, J.G. Rushbrooke, D.R. Ward and T.O. White (Cavendish Laboratory); C. Moore, R. Raja, L. Voyvodic and R.J. Walker (Eermilab); W. Morris, B.Y. Oh, D.L. Parker, G.Z. Smith and J. Whitmore (Michigan State Univ.)

It is proposed to study multiparticle production at the highest available momentum in antiproton-proton interactions in the 30-inch hydrogen bubble chamber using the upstream tagging and the downstream F.W.C.'s. The antiprotons will be produced indirectly in $\Lambda^{\circ}$ decays. Directly produced charged secondaries will be swept away using a dipole magnet near the target. Selecting particles at 160 or $200 \mathrm{GeV} / \mathrm{c}$ depending on whether 400 or $500 \mathrm{GeV}$ incident protons are available should yield adecuate fluxes of particles in a beam containing $\sim 20 \%$ $\bar{p}$ 's. A_total of 100,000 pictures containing about 10,000 useful $\bar{p}$ interactions is requested.

In this experiment all $\bar{p}$ events would be measured and comparison_made with lower energy data (especially from E311$100 \mathrm{GeV} / \mathrm{c} \overline{\mathrm{p} p})$. The analysis would include.

1. $\bar{p}$ topological cross-sections and moments of the multiplicity distribution.

2. Inclusive single particle distributions including particles, $\pi^{c}$ 's and resonances.

3. Two particle correlations and cluster analysis.

4. Exclusive processes_(for_example we expect -200 events of the type pp $\left.\rightarrow \overline{p p} \pi_{\pi}^{+}\right)$.

5. Diffractive processes.

6. Investigation of the $\bar{p}$ annihilation contribution to various processes by comparing with pp data.

Summary revised by

W. W. Neale, January 1976 
PROPOSAL TO STUDY THE PROPERTIES OF THE FORWARD-GOING PHOTON ENERGY IN THE TWO-CHARGED PRONG TOPOLOGY CREATED IN $150 \mathrm{GeV} / \mathrm{C}$ $\pi^{-}-\mathrm{p}$ INTERACTIONS

Brown University, Fermilab, IIT, University of Illinois, Indiana University, Johns Hopkins University, MIT, Oak Ridge, Rutgers University, Stevens Institute, University of Tennessee, Yale University, Padova/Rome Irwin A. Pless (Spokesperson)

It is proposed to study the forward electromagnetic energy associated with the two-charged prong topology in $150 \mathrm{GeV} / \mathrm{c}^{-} \mathrm{p}$ interactions. It is believed that there is evidence of a new phenomenon associated with these photons.

In E154, when beam fragmentation events are examined, an unexpected distribution is observed in the momentum of the $\pi$; there are accumulations of events at 10,40 and $110 \mathrm{GeV} / \mathrm{c}$. These peaks are also reflected in peaks in the energy distributions of the missing neutrals (beam fragments) which pass through the gamma ray detector. There are accumulations at 100 and $140 \mathrm{GeV} / \mathrm{c}$. The questions to be answered are: What is the composition of the neutral energy? Does the accumulation at $140 \mathrm{GeV} / \mathrm{c}$ consist of a single $\pi^{\circ}$ or several $\pi^{\circ}$ 's? What are the invariant mass distributions between the neutral $\pi^{\circ}$ or $\pi^{\circ} \mathrm{s}$ and the $\pi^{-}$? Efforts to explain this phenomenon in terms of known reactions such as $A^{-}$production, have been unsuccessful.

A gamma ray detector would be added behind the last PWC plane in the FNAL 30" hydrid system. This gamma ray detector should have an energy resolution $\frac{\Delta E}{E}<18$ and a spatial resolution of less than $\pm 3 \mathrm{~mm}$. A total of $10^{6}$ expansions is requested in the $30^{\prime \prime}$ hybrid system with an incident $\pi^{-}$beam of $150 \mathrm{GeV} / \mathrm{c}$. The flash will be triggered when an electromagnetic shower energy greater than $20 \mathrm{GeV} / \mathrm{c}$ is detected. It is expected to take 100,000 pictures of which 9,000 will contain two-prong events and of which 3,000 will be the events of interest. This will be an increase of more than an order of magnitude over present data and in addition will have precise information on the proton energy and distribution. This precise information on the photons should allow the experimenters to determine what the character of the beam diffraction reaction is and whether or not it represents a new phenomenon.

In addition, we could take these pictures in an untriggered mode, using a software trigger in the post analysis. This would then be, in addition, a very high statistics experiment at a single energy and single particle. 
PROPOSAL FOR A HIGH STATISTICS STUDY OF ANTIPROTON-PROTON AND PROTON-PROTON INTERACTIONS AT $100 \mathrm{GeV} / \mathrm{C}$ WITH THE FERMILAB 30-INCH HYDROGEN BUBBLE CHAMBER SPECTROMETER AND EXTERNAL PARTICLE IDENTIFIER

R. E. Ansorge, J. R. Carter, W. W. Neale, J. G. Rushbrooke, D. R. Ward and T. O. White (Cavendish Laboratory), C. Moore, R. Raja, L. Voyvodic and R. Walker (Fermilab), B. Y. Oh, M. Pratap, G. A. Smith and

J. Whitmore (Michigan State University), G. Ekspong, S. 0. Holmgren, S. Nilsson and N. Yamdagni (University of Stockholm), plus additional U.S. and European institutions to be added later.

On the basis of our current analys is of E-311 (100 K pictures of $100 \mathrm{GeV} / \mathrm{C} \overline{\mathrm{p}}$ ), we propose to continue our studies of multiparticle production at high energies and request $10^{6}$ pictures of a $100 \mathrm{GeV} / \mathrm{c} \overline{\mathrm{p}} / \pi^{-}$beam and $10^{6}$ pjctures of a $100 \mathrm{GeV} / \mathrm{c} \mathrm{p} / \pi^{+}$beam into the Fermilab 30-inch Bubble Chamber spectrometer. Such a configuration of beams will result in significant studies of both $\pi$ and $p(\bar{p})$ interactions and thus is an efficient use of the facility. An important feature of this proposal is the planned use of a downstream External Particle Identifier (EPI) for $\mathrm{p} / \mathrm{K} / \pi$ separation to identify as many produced particles as possible. The system we propose is the CERN EPI which, with only a few minor modifications, is well suited for the proposed configuration at Fermilab. This system is based on detecting the relativistic rise of ionization loss in gases and successfur tests have been made at CERN. The system consists of 128 layers of 32 individual proportional cells. Ionization deposited in the detector is sampled by measuring the pulse height from each cell traversed by the charged particles. Monte Carlo calculations, substantiated by the tests, indicate that one can obtain $5.5 \%$ FWHM ionization resolution on a track which passes through al1 128 layers. The EPI is capable of separating $\pi$ 's and $K^{\prime} s$ of $\sim 3$ to $-40 \mathrm{GeV} / \mathrm{c}, \pi$ 's and $\bar{p}^{\prime} s\left(p^{\prime} s\right)$ from -3 to $-100 \mathrm{GeV} / \mathrm{c}$ and $K^{\prime} s$ and $\bar{p}^{\prime} s$ ( $p^{\prime} s$ ) from -6 to $\sim 70 \mathrm{GeV} / \mathrm{c}$ with efficiencies of $>90 \%,>90 \%$ and $>80 \%$ respectively.

In earlier considerations of an improved hybrid system for the 30-inch bubble chamber, the concept of particle identification was recognized as an important feature of the system (see Dec. 1975 NALREP). Our studies indicate that the EPI will fulfill the requirements of particle identification for a broad class of experiments at Fermilab. with minor adjustments to the plan of Plano et al (12 Sept. 1974), the overall requirements of a good hybrid system (precision momentum measurements, $\gamma$-ray detection and particle identification) may be readily met.

On the basis of $E-311$ events, we find significant differences between $\bar{p} p$ and $p p$ interactions. It is tempting to ascribe such effects to the baryon annihilation process. For any future high statistics exposures such as proposed here, it is extremely important to have more detailed information on the nature of the produced particles. With the proposed system we expect to study the following topics: (a) a measurement of the $\bar{p}$ annihilation cross section, a study of the multiparticle production in baryon annihilation events, and a high statistics comparison between $\bar{p}$ and $\mathrm{pp}$ interactions. (b) a study of the $K_{\pi}$ correlations in both $\overline{p p}$ and $\mathrm{pp}$ interactions. One of the interesting features observed at Fermilab is the strong dependence of two particle correlations on the azimuthal separation for a pair of 1 ike pions." It would be interesting to learn whether this effect is due to the Bose statistics of two identical particles. One may learn more about such effects by studying $K^{ \pm} \pi^{ \pm}$correlations in a similar manner. With the identification of $K_{S}, K^{ \pm}, \Lambda$ and $\vec{\Lambda}$ in a large number of events we expect to study the process of strangeness exchange in both $\overline{\mathrm{pp}}$ and $\mathrm{pp}$ interactions. For example, what is the rapidity gap distribution between the 2 strange particles? How locally is strangeness conserved? (c) a study of the low cross section processes such as exclusive channels and neutral particle production. (d) with the proposed beams, many of above topics will also be studied in $\pi^{ \pm} p$ reactions. 


\section{CALORIMETER-ARRAY STUDY OF HIGH $P_{T}$ EVENTS}

Fermilab-Lehigh-Penn-Wisconsin Collaboration

W. Selove (Spokesperson)

An experiment is proposed to study high-pt events with a calorimeter array of advanced design and of intermediate size relative to $E-246$. The objectives include detailed study of single- and multiple-particle high- $p_{t}$ events with an individually segmented detector. Major specific objectives are the measurement of :

1. frequency of $h i g h-p_{t}$ groups relative to high- $p_{t}$ single-particle events,

2. total- $p_{t}$ dependence for such groups,

3. some angular correlation information in a two-arm system.

(These objectives are discussed in more detail in the E-246 Proposal.) The detector will consist of a hadron calorimeter on one side of the beam--27 elements of 0.06 to $0.1 \mathrm{sr}$ each--. and a "$\pi$ " hodoscope of 20 elements on the other side. Emphasis is placed, in the detector, on obtaining the cleanest possible identification of very rare events, to a level of one event in $10^{9}$ to 1010 beam tracks. With a beam of $200 \mathrm{GeV} / \mathrm{c} \mathrm{p} / \pi$, $10^{6}$ per pulse, a substantial number of events can be obtained out to at least $5 \mathrm{GeV} / \mathrm{c} \mathrm{p}_{t}$.

Running time requested is 450 hours total, consisting of 150 hours testing and 300 hours data taking. Data taking is planned as 200 hours at $200 \mathrm{GeV} / \mathrm{c}$ and 100 hours at $300 \mathrm{GeV} / \mathrm{c}$. 


\section{ELASTIC SCATTERING AND DIFFRACTION DISSOCIATION AT SMALL MOMENTUM TRANSFER FOR $\pi^{ \pm}, \mathrm{K}^{ \pm}, \mathrm{p}^{ \pm}$AND $\mathrm{n}$}

\section{R. Cool, K. Goulianos, S. Segler, H. Sticker (Rockefeller Univ.)}

A simple apparatus is proposed for measuring the energies, angles, and identity of recoil $p$ or $d$ at small |t| from a gas target. Large solid angle coverage and target length are capable of extending $\mathrm{pp}$ and pd gas jet measurements to $\pi^{ \pm}, K^{ \pm}, \bar{p}$ and $n$ using typical Fermilab existing beams. In addition, the recoil detector will label each diffractively produced state with ' $x^{\prime}$ ' $|t|$ and $f$, the normal to the production plane, for study by downstream apparatus. Since diffractively produced states at very low $|t|$ carry the same quantum numbers as the incoming particle, each final state of mass $M_{X}$ is labeled as to isospin, parity and strangeness. The variety of quantum numbers provided by existing beams then allows a sensitive method of searching for new particles within specified mass ranges with specific quantum numbers. Apart from the particle search potentiality, the systematic study of the important diffraction dissociation production process itself with a variety of incoming particles is emphasized. In addition to measuring $d^{2} \sigma / d M_{x}{ }^{2}|t|$ as a function of $s,|t|$ and $M_{x}{ }^{2}$ for inelastic processes, $d \sigma / d|t|$ for elast $\underset{\sim}{i c}$ scattering will be measured in the range $0.01<|t| \approx 0.12(\mathrm{GeV} / \mathrm{c})^{2}$ thus giving the ratio of the real to the imaginary amplitudes.

A hydrogen (deuterium) gas target at atmospheric pressure is located between planes of proportional and drift chambers and scintillation counters; these measure the recoil proton (deuteron). With a beam of $5 \times 10^{6}$ particles/pulse, a total interaction rate of $86 / \mathrm{sec}$ is expected for pp interactions of which 5 would be detected elastic events. Some $4 \times 10^{5}$ elastic events and $4 \times 10^{4} / \mathrm{M}_{\mathrm{x}}^{2}$ diffraction dissociation events per $(\mathrm{GeV}){ }^{2}$ bin at $M_{X}{ }^{2}$ are expected in one day, spread over incident $\pi ' s, K ' s$ and $\mathrm{p}^{\mathrm{s}}$. The error introduced by coulomb scattering ranges from $\Delta \mathrm{M}_{\mathbf{X}}=100 \mathrm{MeV}$ at $\mathrm{M}_{\mathrm{X}}=1.5 \mathrm{GeV}$ to $\Delta \mathrm{M}_{\mathrm{X}}=20 \mathrm{MeV}$ at $\mathrm{M}_{\mathrm{X}}=7.5 \mathrm{GeV}$.

Phase I of this experiment would have a simple downstream detector to record the number and position of charged particles and $\gamma$-rays in a plane perpendicular to the beam $\approx 10$ meters from the recoil detector. Phase II would add a magnet and $\mathrm{pb}$ glass (or calorimeter) detectors for momentum (and energy) determination in the downstream arm.

For Phase I, 1,000 hours are requested in a beam like Ml or M6. The goal is to measure (i) $\mathrm{d}^{2} \sigma / \mathrm{d}|\mathrm{t}| \mathrm{dM}_{\mathrm{x}}{ }^{2}$ for $50<\mathrm{s}$ $<400 \mathrm{GeV}^{2}, 0.01 \approx|t| \gtrless 0.12(\mathrm{GeV}){ }^{2}$ and $\mathrm{M}_{\mathrm{p}}{ }^{2}<\mathrm{M}_{\mathrm{x}}{ }^{2}<0.15 \mathrm{~s}$ for each particle type, (ii) do/d|t| for elastic scattering over the same ranges of $s$ and $t$ for each particle type and (iii) multiplicities and angular distributions for charged particle and $y$-ray secondaries of diffraction dissociation states together with $\gamma$-ray energies. The apparatus can be ready in early 1976, and its density is so low it can be placed upstream of other experiments. 
PROPOSAL TO SEARCH FOR HIGH MASS PARTICLES PRODUCED IN ASSOCIATION WITH PROMPT MUONS

E. Bleser (Fermilab); B. Gobbi, L. Kenah, J. Keren, R. Lipton, D. Miller, J. Rosen, R. Ruchti, D. Spelbring (Northwestern University); J. Biel, D. Chaney, T. Ferbel, P. Slattery, D. Underwood (Univ. of Rochester); D. Freytag (SLAC)

It is proposed to investigate the possible existence of narrow high mass states (e.g. charmed particles) which are produced in association with prompt muons. Most estimates of the expected magnitude of prompt muon production, which are based on electromagnetic processes or postulated intermediate states involving the known vector mesons, fail to saturate the observed $\mu / \pi$ ratio of $\sim 10^{-4}$. This suggests that a significant fraction of these muons could result from weak decays of heretofore undiscovered particles which are forbidden to decay strongly by a new conservation law. Such particles would of necessity be produced in pairs, and it is proposed to employ the muon resulting from the leptonic or semi-leptonic decay of one state to trigger a spectrometer system, and then to examine the characteristics of the associatively produced particles using a modified version of the E27A/E 305 spectrometer.

The muons are to be identified by their traversal of a steel and heavimet telescope which will be instrumented with counters, proportional chambers, and wire spark chambers. The addition of this second spectrometer arm will permit exploitation of the proven capabilities of the wide aperture, high resolution, forward spectrometer to study the multiplicity, transverse momentum, and invariant mass distributions of events produced in association with prompt muons. In addition, the ability to identify forward leptons will enable the experimenters to investigate $\mu \mu$ and $\mu$ e correlations in these events.

This proposal is a natural outgrowth of the experience gained during a previous search for charm-type particles (revised Experimert 305), and will be a particularly sensitive probe into this obviously important and still relatively unexplored physical domain. A total of 1000 hours of beam time is requested for these studies. 
A PROPOSAL FOR A FURTHER STUDY OF

MUON NUCLEON INELASTIC SCATTERING AT FERMILAB

\author{
Chicago, Harvard, Illinois, Oxford Collaboration \\ H. L. Anderson (Spokesperson)
}

A further investigation of inelastic muon-nucleon scattering is proposed employing the muon spectrometer built by the E-98 collaboration. Specifically it is proposed:

1. To extend measurements of inclusive muon inelastic scattering cross-sections to higher incident energies and thereby study the nucleon structure in kinematic regions not hitherto reached. Compared to E-98, the range of $\mathrm{q}^{2}$ will be extended from $30 \mathrm{GeV}^{2}$ to $50 \mathrm{GeV}^{2}$, and $\nu$ from $130 \mathrm{GeV}$ to $205 \mathrm{GeV}$. The accuracy will be 5 to 108 , depending on binning. This determination depends on assuming a value of $R$, the ratio of the scaler to transverse virtual photon cross-sections. Values of $R$ can be obtained from the overlapping $\left(q^{2}, v\right)$ regions of the proposed $225 \mathrm{GeV}$ runs and the 100 and $150 \mathrm{GeV}$ data of E-98. Values of the total photon-nucleon cross section will be obtained by extrapolation to $q^{2}=0$. This experiment would yield measurements for photon energies from 175 to $205 \mathrm{GeV}$, compared to 90 to $130 \mathrm{GeV}$ in E-98.

2. To identify hadrons and thereby measure $\pi / K / p$ ratios as a function of kinematic variables and charge dependence. preliminary results from E-98 indicate that the charged hadron ratio $h^{+} / h^{-}$is close to 1 for low $x$ and increases as $x$ approaches 1 and that it increases at $q^{2}$ near $x=1$.

3. To extend the measurements of vector meson production to higher energies. This experiment would yield $2000 \rho$ mesons and $100 \rho^{\prime}(1600)$ mesons in the range $\nu=175$ to $205 \mathrm{GeV}$; this compares to $50 \rho^{\prime}$ and $1000 \rho$ mesons in E-98.

4. To investigate the target fragmentation region. This new work is made possible by the cylindrical chambers and will allow measurement of charged particle multiplicities and direct correlations in the target fragmentation region.

A few modifications to the E-98 equipment would be made for this experiment. The more significant of these are: a set of four coaxial cylindrical multi-wire proportional chambers around the hydrogen/deuterium target, a thinner target housing, a $10 \times 10$ element lead-glass hodoscope to measure the forward going electrons and photons and a reduction in the amount of matter in the muon beam by adding helium bags and reducing scintillation counter thickness.

Two periods of running are requested at the pighest momentum available, preferably $225 \mathrm{GeV} / \mathrm{c}$. A total of $2 \times 1011$ muons are required for $\mu \mathrm{p}$ scattering and $2 \times 10^{11}$, for $\mu \mathrm{D}$ scattering. Each run could be completed in 400 hours of real beam time at current intensities. 
PRODUCTION OF ELECTROMAGNETIC CASCADE SHOWERS

BY SEVERAL HUNDRED GEV ELECTRONS IN ENULSION CHAMBERS

R.I. Golden (Johnson Space Center); R.J. Wilkes, J.J. Lord (University of Washington); J. Nishimura (University of Tokyo)

It is proposed to expose two different types of emulsion chambers in order to study the development of electromagnetic cascade showers. One chamber will be composed of a stack of pure nuclear emulsions wherein the showers can be studied in a uniform medium having a radiation length of about $2.9 \mathrm{~cm}$. Comparison will be made with theory and cosmic ray studies.

Experiments will also be conducted with emulsioil chambers consisting of a combination of lead sheets and nuclear emulsions of a design similar to those employed in cosmic ray experiments. Again comparison will be made with theory and the results of cosmic ray investigations.

It is proposed to expose the stacks to electrons of the highest practical energy; at least $100 \mathrm{GeV}$.

Summary prepared by

R. Golden, April 1975 
A SEARCH FOR NEW PARTICLES PRODUCED IN ASSOCIATION WITH THE HIADRONIC PRODUCTION OF $\psi(3.1)$ MESONS

M. Binkley, I. Gaines, J.R. Orr, J. Peoples, A.L. Read (Fermilab); G.E. Gladding, M.F. Gormley, R. Messner, A. Wattenberg (Univ. of Illinois)

The primary objective of this experiment is a search for production of new (possibly charmed) particles from interactions of $400 \mathrm{GeV}$ primary protons with a beryllium target. A suggestion has been made to explain the large increase in the production cross section for the $\psi(3.1)$ corresponding to the accelerator energy increase from BNL-AGS to Fermilab. Consequently, it is speculated that the favored production of the $\psi(3.1)$ is in association with other new particles containing a charmed quark.

In this Fermilab experiment it is proposed to look at the following reaction:

$\mathrm{p}+\mathrm{Be}-\psi(3.1)+\mathrm{Y}_{\mathrm{f}}(\mathrm{A}, \ldots)+\mathrm{X}, \psi(3,1)+\ell^{+} \ell^{-}$.

Here the $\psi(3.1)$ is observed through its decay to a lepton pair and the remaining particles produced and observed in the reaction $\left(Y_{f}\right)$ are examined for narrow peaks in an effective mass plot. These possibly new particles are designated as " $A$ " in the reaction above. The particle A may also be observed through its leptonic decay if it has a significant branching ratio.

A secondary physics objective is to measure the yields and to study the dynamics for production of $\rho^{\circ}(770), \phi^{\circ}(1020), \psi^{\circ}(3100)$, $\psi^{\circ}(3700)$. Similar measurements from Experiment \#358 (involving the experimenters associated with this proposal) do not enable a simple interpretation for these production processes because of complications resulting from use of neutrons which were not monoenergetic. The proton data will make it possible to obtain cross sections from the E-358 data.

It is planned to make several changes to the detector and to the beam to facilitate this experiment. The incident beam is to be $400 \mathrm{GeV}$ protons, reduced in intensity by collimation from about $3 \times 10^{9}$ to $1 \times 10^{7}$, transported through the empty deuterium filter for the wide band photon beam. The detector previously used for Experiments \#87A and \#358 will be changed as follows: 1) increasing in the acceptance by a factor of 4 by moving the target closer, 2) improving the momentum resolution of the measured secondaries by raising the field in the analysis magnet to $18 \mathrm{kG}, 3$ ) improving the lepton detection efficiency by adding counters, reducing the length for possible $\pi$-decay, and by improving the muon filter.

The experimenters ask for 50 hours of running to study the possibilities of bringing a proton beam to their apparatus. Later running would include 100 hours for testing, 600 hours for data-taking, followed by 120 hours of data-taking with additional steel inserted in their apparatus to study carefully backgrounds from pion decays.

Summary prepared by

A. F. Greene, June 1975 
R. Coleman, G. Gladding, M. Goodman, M. Gormley, R. Messner,

A. Wattenberg (Univ. of Illinois); M. Binkley, I. Gaines,

J.R. Orr, J. Peoples, A.L. Read (Fermilab)

It is proposed to extend the photoproduction studies begun in Experiment \#87A. The measurements planned for these new studies are:

1) measurement of the energy dependence of $(\mathrm{d} \sigma / \mathrm{dt})$ for $\psi(3.1)+$ nucleon $\rightarrow \psi(3.1)+$ nucleon, $t=t_{\min }$

2) measurement of the $\psi^{\prime}(3.7)$ + nucleon cross section,

3) investigation of the mass spectrum of photoproduced $e^{+} e^{-}, \mu^{+} \mu^{-}, K^{+} K^{-}, p p, \mu e$, etc. in the mass interval $3.0 \mathrm{GeV} \leq \mathrm{M}_{+-} \leq 7.0 \mathrm{GeV}$.

It is emphasized in the proposal that the determination of the $\psi^{\prime}(3.7)+$ nucleon cross section can probably only be measured at Fermilab energies in such an experiment. Furthermore, a study of the photon-induced two-particle mass spectrum (both resonant and non-resonant) is a natural extension to the earlier measurements of Experiment \#87A. The (cross section) $x$ (branching ratio) for a mass $6 \mathrm{GeV}$ object is expected to be about 1 nanobarn.

It is planned to make some additions to the setup for Experiment $87 \mathrm{~A}$. These are expected to include:

1) an additional sweeping magnet in the form of a BM-109 to remove $e^{+} e^{-}$pairs with momentum less than $25 \mathrm{GeV}$ just downstream of the experimenters' target,

2) addition of one or 2 Cerenkov counters behind the analyzing magnet for the present E87A apparatus thus to reduce the number of events with secondary pions,

3) improvement of the existing electron/photon identifier.

The main features of these improvements will be a factor of two improvement in the mass resolution (from $145 \mathrm{MeV} F W H$ to about $82 \mathrm{MeV}$ FWHM), a factor of ten improvement in sensitivity, and acceptance of events with a broader range of incident photon energies.

Event rates are calculated using an extracted proton beam with an intensity of $5 \times 10^{12}$ per pulse and an energy of $300 \mathrm{GeV}$. In a 300 hour run the yield of events in the mass region near $5 \mathrm{GeV}$ would be about 1,200 events and near $7 \mathrm{GeV}$ would be 250 events. If the primary proton beam energy is at $400 \mathrm{GeV}$ the number of events in the mass region $3-5 \mathrm{GeV}$ would increase by a factor of 1.5 to 2 . 
$\pi^{+}$p INTERACTIONS AT $250 \mathrm{GeV} / \mathrm{c}$ IN IMPROVED FNAL 30" HYBRID SYSTEM

University of Tennessee, Oak Ridge National Laboratory, John Hopkins University, Rutgers University, Stevens Institute of Technology William M. Bugg (Spokesperson)

It is proposed to utilize the excellent momentum resolution, increased angular acceptance, and neutral particle detection capabilities of the improved FNAL 30" hybrid bubble chamber system to study interaction of $250 \mathrm{GeV} / \mathrm{c} \pi^{+}$mesons in an enriched $\pi^{+}$beam. The experimenters will examine leading particle effects and diffraction dissociation, long and short range correlations, inclusive single particle and resonance production, strange particle production, and systematics of events with forward $\pi^{\circ}$ and $\eta^{\circ}$.

Comparison with Experiment 299 results and a proposed experiment at $75 \mathrm{GeV} / \mathrm{c}$ will permit determination of general energy dependence of these effects, particularly leading particle cross section where good resolution near $x=1$ is essential to test carefully model dependent predictions of leading particle cross sections.

An exposure of $100,000 / F$ pictures is requested where $F$ is the fractional $\pi^{+}$content of the beam. With this, it is estimated that $20,000 \pi^{+}$events and 40,000 (1-F)/F proton events will be obtained. The experiment would be of sufficient statistical accuracy to permit detailed comparison with other proposed experiments using the improved 30 " hybrid system. 
HYBRID BUBBLE CHAMBER STUDY OF p̄p INTERACTIONS AT $75 \mathrm{GeV} / \mathrm{c}$

Rutgers University-Stevens Institute, Johns Hopkins University, University of Tennessee-Oak Ridge National Laboratory, Stockholm University R. Plano (Spokesperson)

A careful study of antiproton-proton interactions at 75 $\mathrm{GeV} / \mathrm{C}$ is proposed to be made with the Fermilab 30 " hybrid bubble chamber. An exposure of $300 \mathrm{~K}$ pictures is requested to provide $50 \mathrm{~K}$ interactions of which approximately $7 \mathrm{~K}$ are annihilation events. Using the properties of this system which is very well matched to the often complex topologies associated with annihilations and to this beam momentum, the collaborators plan to study all experimentally accessible features. In particular, single and multi-particle inclusive distributions for charged and neutrals will be measured, exclusive fits will be made where possible, new particles will be searched for, and comparisons made to other energies and to other beam particles.

Summary prepared by

R. J. Plano, May 1975 
$\pi^{+}$p COLLISIONS AT $75 \mathrm{GeV} / \mathrm{C}$ IN THE 30-INCH HYBRID CHAMBER

Rutgers University-Stevens Institute, University of TennesseeOak Ridge National Laboratory, Johns Hopkins University T. L. Watts (Spokesperson)

It is proposed to take a more detailed look at $\pi^{+} p$ collisions at an energy intermediate between AGS energies and current high energies at Fermilab. The experimenters would initially concentrate on the quasi-elastic diffraction processes to understand their energy dependence by comparison to the data at $150 \mathrm{GeV} / \mathrm{C}$ (Fermilab experiments E154 and E299). They expect to examine the following features of leading particle interactions especially concentrating on their energy and beam particle dependences:

(a) Leading particle cross-sections

(b) Associated multiplicity

(c) Comparative $\pi^{+}, \pi^{-}$and $\mathrm{p}$ inclusive distributions. Correlation among particles, i.e. invariant masses, azimuthal angles, longitudinal and transverse momentum distributions. They expect to look for resonances and sub-clustering effects.

(d) Exclusive final states determined by $4 \mathrm{C}$ fits

(e) $\pi^{\circ}$ production using conversions in the bubble chamber or in a lead glass hodoscope which the experimenters plan to help make available.

(f) Strange particle production.

The exposure is for the pure beam equivalent of $100 \mathrm{~K}$ pictures in the 30" hydrogen bubble chamber hybrid system with proportional wire chambers to isolate the fast leading particles.

Summary reviewed by

T. L. Watts, January 1976 


\section{HYBRTD BUBBLE CHAMBER STUDIES OF $\mathrm{K}^{+} \mathrm{p}$ AND $\mathrm{K}^{-} \mathrm{p}$ INTERACTIONS AT $75 \mathrm{GeV} / \mathrm{C}$}

Johns Hopkins University, Rutgers University-Stevens Institute, Stockholm University, University of Tennessee-Oak Ridge National Laboratory A. Pevsner (Spokesperson)

An exposure of the Fermilab 30" liquid hydrogen bubble chamber to $\mathrm{K}^{+}$and $\mathrm{K}^{-}$beams both at $75 \mathrm{GeV} / \mathrm{c}$ is proposed. Each exposure consists of 100,000 equivalent $\mathrm{K}$ pictures, i.e. $100,000 / F$ pictures each, where $F$ is the enrichment factor. This would yield 10,000 events for each, $\mathrm{K}^{+}$and $\mathrm{K}^{2}$.

The experimenters plan to use the upstream tagging system currently in place and a downstream spectrometer with acceptance considerably increased over that of the current PWC system. This new downstream system will also be equipped with a lead glass photon detector with good spatial and energy resolution.

A comparison will be made of $\mathrm{K}^{+}$and $\mathrm{K}^{-}$results, as well as results from $\pi^{+}, \pi^{-}$, and $\bar{p}$ beams at this energy which are the subject of separate proposals. In particular they plan to examine:

1. Particle multiplicities.

2. Leading particle effects and diffraction dissociation.

3. Double leading effects and long range correlations.

4. Strange particle production.

5. Resonance production.

6. Multiparticle production in the central region. 
PROPOSAL TO SEARCH FOR PION RRORUCED PARTICLES DECAYILE UNDER PROMPT ENISSIONS OF ONE OR NORE NUONS

D. Garelick, M. Glaubman, E. von Goeler, R. Weinstein, P. Gauthier, M. Mallary, D. Potter, E. Pothier of Northeastern University; and P. Mockett, G. Hicks of the University of washington, and others

This experiment will search for the pyociction of new particles which promptly decay emitting one or more muons. The detectors for this experiment consist of two spectrometers: A spectrometer which measures slow recoil protons produced at large angles with respect to the beam and an iron spectrometer which identifies and measures muons produced in the forward direction $\left(\theta_{\mathrm{H}} \sim \theta_{\mathrm{V}} \sim 0.2 \mathrm{rad}.\right)$.

Some of the processes that will be investigated are: (1) the production of fairs of particles such as charmed mesons, $D$, produced in reactions $\pi^{-}+p \rightarrow D+D+p$ or $\pi^{-}+p \rightarrow D+\bar{D}+$ hadrons $+p$, where at least one $D$ decays under muon emission. Another process of this type is heavy lepton production. (2) the production of particles, $w^{-}$, produced singly in reactions $\pi^{-}+p \rightarrow w^{-}+p$ or $\pi^{-}+p \rightarrow W^{-}+p+$ hadrons, where the $W^{-}$ decays under muon emission, for example $W^{-} \rightarrow \mu \nu$ or $W^{-} \rightarrow \mu x$. (3) the production of particles, $x$, produced in reactions $\pi^{-}+p+x^{-}+p$ where the $x$ decays under emission of at least one vector meson, $V$, which in turn decays into a muon pair. (4) multimuon production by $\pi^{-}, K^{-}$, and $\bar{p}$ from an iron target.

The unique features of the experiment are: (1) use of a missing mass technique to obtain the spectrum of masses $D \bar{D}, \bar{D} \bar{D}+$ hadrons, $W^{-}, W^{-}+$hadrons or $x^{-}$. This technique allows a rapid mass scan for masses up to $\sim 10 \mathrm{GeV}$, and with good resolution, $\Delta \mathrm{M}^{2} \sim \pm 1 \mathrm{GeV}^{2}$. (2) use of a double missing mass technique to determine the nature of the intermediate state from which the muon (or muons) originate. (3) Excellent cross section sensitivity in the nanobarn region. (4) Tesi ciata exist which have determined background levels and have shown the feasibility of the experiment. (5) Most of the apparatus exists. (6) The ability to collect interesting data on multimuon production by $\pi^{-}, K^{-}$, and $\bar{p}$ on an iron target.

The experiment requires 200 hours in a $200 \mathrm{GeV} \pi^{-}$beam with a flux of $\sim 10^{7} /$ pulse. In addition, approximately 150 hours of parasitic time for apparatus testing and calibration are needed. 
PROPOSAL TO SEARCH FOR PROTON PRODUCED PARTICLES DECAYING UNDER PROMPI EMISSION OF ONE OR MORE MUONS

W. Faissler, D. Garelick, M. Glaubman, J. Johnson, M. Mallary, E. Pothier, D. Potter, M. Ronan, E. von Goeler, R. Weinstein (Northeastern University)

We propose to measure the reactions

$$
\begin{array}{r}
p+p+x^{+}+p \rightarrow \mu+x^{\prime}+p \\
\text { or } \mu \mu+x^{\prime}+p
\end{array}
$$

to examine possible structure in the $x^{+}$mass spectrum. In this experiment a slow proton at large angle and one or more muons in the forward direction are detected. of the type

$$
\text { Such structure might arise, for example, from a reaction }
$$

$$
\mathrm{p}+\mathrm{p} \rightarrow \mathrm{DB}_{\mathrm{C}} \mathrm{p}^{\rightarrow} \mu \mathrm{X}^{\prime} \mathrm{p} \text {. }
$$

Here ${ }^{B}$ might be a charmed baryon, $D$ a charmed meson. Either particle could decay and give rise to one or more muons in the detector. The experiment is similar, except for the incident particle, to Northeastern University proposal 365A, and should follow that experiment.

The experiment requires 200 hours of beam time with $10^{7}$ protons per pulse at $300 \mathrm{GeV}$ or $400 \mathrm{GeV}$. The sensitivity of this experiment to reactions of type (2) is approximately $5 \mathrm{nb}$. Test data have already been taken and have shown the feasibility of the experiment. For further details, see Northeastern University Proposal 411 and references cited therein.

Summary prepared by

D. Garelick, May 1975 


\section{MEASUREMENTS OF ${ }^{-} \mathrm{CU}+\mathrm{K}_{\mathrm{S}}^{\circ}, \Lambda^{\circ}, I_{\mathrm{IND}}$ \\ NEUTRON INCLUSTVE CROSS SECTIONS}

University of Colorado, University of Michigan, Rutgers

University, University of Wisconsin

\section{Fondrom (Spokesperson)}

It is proposed to measure inclusive strange particle yields from a pion bombardment of a copper target. This work is motivated by the inherent physics interest as well as by a need for particle production data in order to design a $\mathrm{kl}$ beam in the proton-West area. The design work is motivated by interest in Proposal \#360 to study inclusive interactions of $\mathrm{k}_{\mathrm{L}}^{\circ}$ producing hadrons and leptons. The $\mathrm{K}_{\mathrm{L}}^{\circ}$ beam would be procuced by a high energy, high intensity pion beam and thereby contain a reduced number of background neutrons.

This experiment would use the M2 beam of the Meson Area set for $\pi^{-}$at a momentum of $200 \mathrm{GeV} / \mathrm{C}$ and at much lower intensity than that planned for P-West. As a detector the experimenters would use the apparatus of Experinent \#8, a neutral "V" spectrometer. KO's would be identified through their decay to $\pi^{+} \pi^{-}$, $\Lambda^{\circ}$ through their $\mathrm{p \pi}^{-}$mode, and neutrons through a technique utilized in a neutron dissociation experiment (E-305). Neutron production can be identified by scattering in a carbon target, producing $\mathrm{N}^{\star \prime} \mathrm{s}$ which decay to $\mathrm{p} \pi^{\text {' }}$.

The running time request for this experiment is 100 hours.

Summary prepared by

A. F. Greene, June 1975 
V. Cook, S. Csorna, A.M. Jonckheere, R.G. Kenyon, H.J. Lubatti.

K. Moriyasu, B. Robinson, P. Tressel (Univ, of Washington);

D. Fournier, F. Feusse, P. Pancon, J.J. Veillet (Orsay);

J. Erwin, R. Kass, J.H. Klems, Winston Ko, R.L. Lander,

D.E. Pellet, P.M. Yager (Univ. of California, Davis)

A test has been made to determine if an enhancement in neutral strange particles ( $\mathrm{V}^{\circ} \mathrm{S}$ ) observed in a streamer chamber exists when triggered on muons. The beam of $225 \mathrm{GeV}$ negative pions was incident on a $1.3 \%$ lucite target.

The reactions being compared were:

$$
\begin{aligned}
& \text { (1) } \pi^{-} \mathrm{N}+2 \mu+\text { vo's + anything } \\
& \text { (2) } \pi^{-} \mathrm{N}+1 \mu\left(90^{\circ} \mathrm{cm}\right)+\mathrm{V}^{\circ} \mathrm{s}+\text { anything } \\
& \text { (3) } \pi^{-} \mathrm{N}+\mathrm{V}^{\prime} \mathrm{s}+\text { anything }
\end{aligned}
$$

In reactions (1) and (2) a muon filter (described below) defines a pair of $\mu^{\prime}$ s or $1 \mu$ around $90^{\circ}$ in the c.m. In reaction (3) the trigger is any interaction $(t)$ (we remove the $\mu$ requirement).

The presence of a 3 standard deviation signal is suggestive that we may have seen a real effect. We have tried to find an explanation for this signal which does not assume a new production mechanism or the production of a new particle with a strong semileptonic decay mode. A possible known mechanism is $k \mu_{2}$ decay which would provide a muon and by strangeness conervation would require the presence of an additional strange particle. We have attempted to estimate the contribution from this mechanism. From a simple calculation, we conclude that an upper limit could be approximately 1-2으. A more complicated calculation suggests that this is too large by at least an order of magnitude.

In order to improve the statistical significance of these data, we should like to increase our events by approximately an order of magnitude. We believe that with a 3 -week run this should be possible. In a hundred hours of running, this would give us 15,000 triggers which is a factor of 30 more than we obtained in our previous run. To improve our signal/noise, we plan to use instead of pure iron for the muon filter, the magnetized iron used by Experiment 365. The magnetized iron would al low us to harden the muon trigger by rejecting lower momentum muons, many of which come from pions decaying cither in flight or in the shield. We would in a huncred hours end up with approximately 5,000 events. Improving our trigger by lowering the rate per interaction should decrease some of the background andhhopefully $\alpha_{2 \mu}$ should increase. If this were to be the case, it would be a strong experimental argument that we are observing a new effect. We are encouraged by the results obtained by Perl at SPEAR which suggest that there may be a statc of mass approximately $2 \mathrm{GeV}$ which has a $10 \%$ leptonic decay mode. Such a state may have a substantial semi-leptonic decay mode which would explain its absence in the $k \pi$ mass spectra studied to date. 


\title{
Nuclear Size Dependence for Particle Production at
}

\section{Intermediate Transverse Momentum}

D. A. Garbutt, R. Rusack, I. Siotis (Imperial College); D. Gross,

D. Nitz, S. Olsen (Rochester); K. Abe, R. Bomberowitz, K. Cohen,

P. Goldhagen, F. Sannes, D. Saroff, J. Willison (Rutgers)

In a recent experiment Cronin et al. parametrized the A dependence for particle production from nuclear targets as $A^{n}\left(p_{T}\right)$. Not only does $n$ vary with $p_{T}$ but, most unexpectedly, it takes on values significantly larger than unity. We propose to study this dependence on the atomic number $A$ of $\pi^{+}, K^{-}$and $p^{ \pm}$ production from carbon and tungsten targets at $\theta_{1 \mathrm{ab}}=15^{\circ}$ and $0.25<p_{T}<2.5 \mathrm{GeV} / \mathrm{c}$. The production from both types of targets will be measured simultaneously by mounting carbon and tungsten fibers on the same rotating target at $\mathrm{C}-\mathrm{O}$. Data will be collected over the incident energy range of 30 to $400 \mathrm{GeV}$. Particle momenta will be determined by magnetic analysis and particle species will be determined with one differential and two threshold Cerenkov counters.

\author{
Summary Revised by \\ F. Sannes, Jan. 13, 1976
}




\section{SEARCH FOR SHORT IIVED PARTICLES PRODUCED BY $300 \mathrm{GeV}$ PROTONS IN EMULSIONS}

W. Bozzoli, P. Capiluppi, A. Forino, R. Gessaroli, G. Giacomelli, P. Lugaresi-Serra, A. Quareni and F. Rimondi (Universita di Bolognal; S. Di Caporiacco, A. M. Cartacci and M. G. Dagliana (Universita di Firenze)

This is a search for short-lived particles with lifetimes in the range of $10^{-12}-10^{-14} \mathrm{sec}$., performed with $300 \mathrm{GeV}$ protons in emulsion nuclei. As in experiment No. 462, the signal would be a neutral or charged decay (or possibly two decays) close to the primary proton-nucleus interaction.

We have exposed one stack of Ilford G5 emulsions, of 80 plates of $10 \times 10 \times 0.06 \mathrm{~cm}^{3}$, to $300 \mathrm{GeV}$ protons with a density of about $3 \times 10^{5}$ protons $/ \mathrm{cm}^{2}$, with a tota 1 exposure of $4 \times 10^{6}$ incident protons over an area of $5 \times 8 \mathrm{~cm}^{2}$.

The experiment should be sensitive to the production of new particles if they have a mass of $1-10 \mathrm{GeV}$ and if their production cross section is larger than $1 \mathrm{\mu b}$.

Summary prepared by G. Giacomelii, January 1976 
HIGH PRECISION STUDIES OF DEEP SCATTERING PHENOMENA FROM PROTOIS, PIONS, ELECTRONS AND GAMMA-RAYS ON HYDROGEN AND DEUTERIUM

G. Conger, J. Edighoffer, A. Grigorian, Z.G.T. Guiragossian,

R. Hofstadter, M.R. Yearian (Stanford University)

It is proposed to measure with high precision the cross sections of elastic and inelastic collisicrs in the deep scattering region, using the highest energy and intensity beams of protons, pions, electrons and gamma-rays, on hydrogen and deuterium targets. Initially, these studies will be carried out in the beam momentum region of $50-400 \mathrm{GeV} / \mathrm{C}$, and thcreafter in the Energy Doubler/Savcr mode, in the region of $400-1000 \mathrm{GeV} / \mathrm{c}$. The cross sections of these processes will be measured as a function of $\mathrm{s}$, in the range of 95 $\mathrm{GeV}^{2}$ to $1880 \mathrm{GeV}^{2}$, as a function of the invariant momentum transfer $t$, in the range of $t_{\min }=0.040(\mathrm{GeV} / \mathrm{c})^{2}$ and $t_{\max }=3.25(\mathrm{GeV} / \mathrm{c})^{2}$, and as a function of the missing-mass $M$, in the range of $M=m_{T}$ and $M=40 \mathrm{GeV}$. Beam intensities and energies to be used are: $50-1000 \mathrm{GeV} / 10^{11}-10^{12} \mathrm{p} / \mathrm{pulse} ; 50-800 \mathrm{GeV}, 10^{9}-10^{10} \pi^{-} / \mathrm{pulse}$; 50-700 GeV, $109-1010$ et/pulse; $50-700 \mathrm{GeV} 1 / 2\left(10^{9}-10^{10}\right) \mathrm{y} / \mathrm{pulse}$. Moreover, using high precision missing-mass techniques, searches will be made to discover new states of: heavy baryons ( $\mathrm{N}^{+}$and $\mathrm{N}^{* O}$ ) produced by protons; heavy mesons $\left(M^{*-}\right)$, exotic mesons ( $M^{*--}$ ), and charged intermediate vector bosons $\left(W^{-}\right)$produccd by pions; heavy vector and scalar bosons ( $\left.W 0, V * 0, B * 0, \psi * 0, \eta^{* 0}\right)$ produced by real and virtual photons; excited leptons (e*t), and heavy leptons (EO) produced by electrons.

It is proposed to complete and install the HEPL 168", 2.5 $\mathrm{GeV} / \mathrm{c}$, large acceptance, high precision, focussing spectrometer at Fermilab, at the end of the proton-West beam line, so that it can be used initially for the benefit of these studies. The goal is to complete fabrication of the spectrometer at HEPL in 1978 , and to transport and install the spectrometer at Fermilab in 1979 . Consideration of this proposal is requested presently to permit the completion of the fabrication project.

Summary prepared by Z.G.T. Guiragossian, June 1975 


$$
-259-
$$

EXPOSURE OF AN EMULSION CHAMBER TO A 300 GEV PROTON BEAM.

$$
\text { V.P. Dzhelepov (Joint Institute of Nuclear Research) }
$$

An emulsion chamber $\left(20 \times 10 \times 10 \mathrm{~cm}^{3}\right)$ was exposed to a $300 \mathrm{GeV}$ proton beam. The requested intensity was $1-1.5 \mathrm{x}$ $10^{5}$ protons per $\mathrm{cm}^{3}$.

\author{
Summary prepared by \\ J. Lach, May 1976
}


PROPOSAI, TO STUDY $\overline{\mathrm{p}}$ INDUCED REACTIONS BY MEANS OF THE 30-IN. BUBBLE CHAMBER, I.E. :

$\overline{\mathrm{p}} \mathrm{d}$ INTERACTIONS AT $\sim 50$ AND $\sim 80 \mathrm{GeV} / \mathrm{C}$

$\overline{\mathrm{p}} \mathrm{P}$ INTERACTIONS AT $\sim 80 \mathrm{GeV} / \mathrm{c}$

Centre de Recherches Vucleaires de Strasbourg, Purdue University (A. Fridman, spokesperson)

The proponents request three exposures of the 30-in. Bubble Chamber to an antiproton beam as follows:

1. $150 \mathrm{~K}$ pix in deuterium at $50 \mathrm{GeV} / \mathrm{c}$

2. $150 \mathrm{~K}$ pix in hydrogon at $80 \mathrm{GeV} / \mathrm{c}$

3. $150 \mathrm{~K}$ pix in deuterium at $80 \mathrm{GeV} / \mathrm{c}$

They have acquired substantial $\bar{p}$ data in bubble chamber experiments carried out at lower energies. They list among their interests

for this proposed experiment:

1. Measurements of topological cross sections and statistical moments.

2. Correlations between the production of neutral and charged particles.

3. Search for resonances and study of leading particle effects in non-annihilation channels.

4. Study of multiparticle production.

5. Study of diffractive dissociation in the non-annihilation channels.

6. Single particle distributions in inclusive and seniinclusive reactions.

7. Strange particle production.

8. Total cross section measurements for the annihilation channels.

9. Coherent production processes.

The scanning and measuring will be carried out at both Purdue and Strasbourg. The latter group has indicated they will give top priority to this experiment in committing their analysis resources. 


\section{SEARCH FOR NEW IRPTICLES IN EMULSION CHAMBER}

F. Sugimoto and II. Nanjo (Univ. of Tokyo); Y. Sato (Waseda Univ.); T. Ogata (Kwansai Gakuin University)

The specially ciesigned emulsion chamber with producing layers has the very righ spacial resolution comparea with other particle detectors. The proposal of our experiment is to expose the enulsion chamber to the FNIL 400 GeV/C proton beam to investigate the multiparticle production processes. The main ourvose of our experiment is to search for the new hadronic marticlcs with rear lives in the range $10^{-11}$ to $10^{-14}$ seconds through their decay rechanisns among the multiparticle processes. The momenta of the charged particles are measured by multiple coulomb scattering in lead plates. Leptons are identified alsc in lead plates through the electro-magnetic processes.

The rain objectives of this experiment are:

1. to search for the new hadronic particles through their decay mechanisms and to estimate the cross section of theri,

2. to investigate the angular correlation and also the $\mathrm{N}^{-1} \mathrm{ch}$ correlation of the produced particles in multiparticle processes,

3. to estimate the scattering constant of lead plates in tris chamber by measuring the multiple coulomb scattering of the incident particles.

Conditions Required in this Experiment

1. Beam; parallel and mono-energetic proton beam.

2. Energy; proton beam about 300 and $400 \mathrm{GeV}$.

3. Amounts of irradiation; $* 10^{3}$ and $10^{4}$ particles/cm ${ }^{2}$ or the detector.

4. Shiela materials; lead blocks enough to shield the detector. Euration of the resting and Data-Taking

About two hours in our rough estination.

Suniary prepared Ly

ii. Sugimoto, July la75 


\section{MULTIPLE-PION PRODUCTION BY $200 \mathrm{GeV} / \mathrm{C}$ MUONS}

Tomonori Wada (Okayama Univ.); Shuichi Katsube, Katsuhiko Saitoh (Ashikaga Inst. of Technology); Kiyomi Mitsui (Univ. of Tokyo); Kohei Mizutani (Saitama Univ.)

The main objectives of this experiment are to study the multiple-pion priduction, especially fire ball-like behavior at the known muon energy, and to compare with the cosmic ray data by means of cloud chambers containing lead plates and with emulsion stack data in the lower energy $(5 \mathrm{GeV} / \mathrm{c})$. The cloud chamber data by cosmic ray muons has been investigated by means of the one Fire Ball Model the mesons are emitted from the excited centers) in the energy range of 10 to 100 $\mathrm{GeV}$, while the emulsion data at $5 \mathrm{GeV} / \mathrm{c}$ have indicated dominant contributions from isobars (the excited baryon of $1238 \mathrm{MeV}$ ). In the cosmic ray data we can not discuss more details of "the fire ball", such as its mass or tempcrature, because that incident muon energies can not be estimated directly.

Two detectors are constituted of nuclear emulsion plates and $0.25(0.5) \mathrm{cm}$ lead plates which are piled up in the form of a stack. The geometrical size of the detector is about $10 \mathrm{~cm} \times 10 \mathrm{~cm}$ wide and 16 (12) cm thick including lead target of $8 \mathrm{~cm}$ thick for $300 \mathrm{GeV}$ muons.

Following is the sumary of the conaitions required in this experiment:

1. Beam: parallel and mono-energetic muon beam.

2. Energy : $300 \mathrm{GeV}$

3. Intensity: $10^{5}$ muons $/ \mathrm{cm}^{2}$ on the detector.

4. Accuracy of the beam energy: better than $\pm 10 \%$.

5. Time for data-taking: February 1976 at the first stage. 
PROPOSAL TO INVESTIGATE REGENERATION OF NEUTRAL K-MESONS AT VERY HIGH ENERGIES

N. Molzon, J. Roehrig, V. Tclegci, L. Winstein (Univ. of Chicaco);

S. Aronson, G. Bock (Univ. of Wisconsin); K. Freudenreich,

A. Gsponer (Swiss Inst. of Technology, Zurich); H. Kobrak,

R. Pitt, R. Swanson (Univ. of California, San Diego)

This is the hydrogen target phase of E-82.

Summary prepared by

T. Yamanouchi, May 1976 


\section{EXTENSIVE ANALYSIS OF LIGHT NUCLEI TRACKS IN PLASTIC DETECTOR}

Katsura Fukui (Air Force Geophysic Lab.); R. Beaujean, W. Enge (University of Kiel, Germany)

Some of the sensitive plastic detectors are capable of detecting light nuclei particles such as Li, Be and B. Analysis of these tracks shows clear separation of charge as well as isotopic components. If we apply these detectors to the study of cosmic radiation, however, background tracks created by the interactions of protons and target nuclei in the detector interfere with the analysis of primary light particles and a proper solution is needed. Thus, it is important to study the process of producing sccondary particles irsice nuclei.

For this purpose plastic blocks consisting of Daicel or Kodak cellulose nitrate sheets is exposed to the high energy proton beams. Exposure is made $\in$ ither parallel or perpendicular to the plastic surface, thus angular distribution of secondary tracks may be better analysed. Plastic sheets are etched in a 6 n NaOr solution at proper temperature for several hours. They are scanned under a steromicroscope to find so called Gothic Window cones which represent secondary tracks originated from the collisions between relativistic proton and the target nuclei such as $C$ and 0 ators of the plastic detector. By analysing these tracks, evaporation and spallation process occuring inside the nuclei will be studied. Thus, the nature of background tracks may be clarifick and the criteria of track identification may be established. Consequently, this work will open the way of using plastic as a useful detector for the study of light particles of both galactic and solar origin.

In order to simulate relativistic cosmic ray, $100-300 \mathrm{GeV}$ protons are required for the experiment.

No specific devices is needed for this experiment. Only requirement for the exposure is correct alighment of the plastic sheets with respect to the direction of the beam.

Summary prepared by

K. Fukui, March 1976 
A PROPOSAL FOR TESTING A TRANSITION RADIATION DETECTOR

AND A HIGH ENERGY SHOWER DETECTOR FOR COSMIC RAY EXPERIMENTS

AT FNAL

G.F. Del1, H. Uto, and Luke C.I. Yuan (BNL)

We propose to use a high energy beam containing hadrons and electrons to optimize transition radiation detectors at FNAL energies with particular emphasis on the use of Xe gas, for the use of detectors in the experiments. Measurements of the yield of transition radiation with usual mixture of Argon and methane have already been made earlier at FNAL at lower energies, up to $250 \mathrm{GeV}$. The specific objectives of the experiment are as follows:

1. Measurement of the yield of transition radiation from electrons and hadrons using a large number of proportional wire chambers (PW's) filled with Xe, with different number of foils and chambers up to the highest energy available $(\sim 400 \mathrm{GeV})$ and compare with theoretical estimates. The standard transition radiator is $\frac{1}{2}$ mil Mylar spaced at $30 \mathrm{mils}$. We use also the Ethafoam, 1 mil and $\frac{1}{4}$ mil Mylar with different spacing.

Study of effects of multiple scattering on transition radiation as a function of the beam energy. The multiple scattering may increase the yield of transition radiation.

Study of effects of $\delta$-rays on transition radiation detectors.

2. Study on the practical use of the detector for separating particles, e, $u, \pi, k$ and $p$ using fast chambers, for the accelerator use.

3. Optimization of the transition radiation detector and a high energy shower detector for cosmic ray experiments. The experiments considered are the measurement of the energy spectrum of cosmic ray electrons from a few $\mathrm{GeV}$ to $1000 \mathrm{GeV}$ and the energy spectra of high $z$ relativistic nuclei in cosmic rays.

The specific request from FNAt is for a space of 10 - 15 feet along a beam line in the Meson Area. The running time required is four weeks.

Summary reviwed by

Luke C.L. Yuan, January 1976

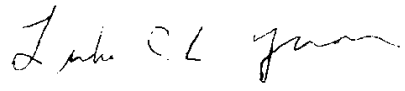


$400 \mathrm{GEV}$ PROTON INTERACTIONS IN NUCLEAR EIIULSION

J. Hebert, C.J.D. Hebert, i. Areti (Ottawa); M.A. Vincent (Quebec);

G. Baumann, R. Devienne (Nancy); J. Iory, C. léton, D. Schune,

E. Nillot, B. Charquet, Tsai-Chu, M. Moranc (Paris); R. Kaiser, J.P. Massue, R. Ffahl, C. Jacquot (Strasbourg); I. Otterlund

(Lund); A. Van Ginneken (Fermilab); J. Juric, O. Adamovic

(Belgrade); R. Schmitt (Lyor); J.M. Bolta, J. Pellicier, G. Rey, F. Tejerina (Valencia); F. Fernandez, J. Medena, A. Dura,

V. Gardia (Valencia). E. Villars (Santanber)

This study extends the work of Experiments 116 and 233 (200 and $300 \mathrm{GeV}$ protons on emulsion and largely completed) to higher energies. The emphasis is on establishing the general characteristics of inelastic p-nucleus interactions: interaction length, average multiplicity (differentiated by track censity), multiplicity and angular distributions, angular and multiplicity correlations, coherent production, etc. Where feasible interactions will be differentiated by target (Ag and $B r$ versus $H, C, N$ and $O$ ).

Results are to be compared with results obtained with emulsion at lower energies, with results obtained with other target elements (including hydrogen), with empirical scaling laws as well as with theoretical predictions.

In the course of these measurements special attention will be given to the possibility of encountering new (or poorly established) short-lived particles.

Summary prepared by

A. Van Ginneken, May 1976 
PROPOSAL TO STLDY IATERACTIONS OF 1 ONO GEV/C NEGATIVE PIONS IN THE 15-FOOT FNAL HYYDPOGEN BUBBLE CHAMBER

Aachen-Bonn-CERA-Cracow-London-larsaw Collaboration

Douglas R.O. Morrison - CERN (Spokesman)

A request is made for 50,000 photographs of $100 \mathrm{GeV} / \mathrm{C}$ negative particles in the 15-foot FNAL hyarogen bubble chamber. One of the main aims of the experiment is to study exclusive reactions. This would greatly extend our experimental knowledge of reaction mechanisms, in particular of diffraction aissociation strange particle production would also be expecially studied.

Sumnary prepared by

D.R.O. Morrison, August 1975 
PROPOSAL TO STUDY $50,000 \quad \Sigma^{-}$n MULTIPRONG EVENTS AT $\sim 240 \mathrm{GeV} / \mathrm{C}$ BY MEANS OF THE 15' BUBBLE CHAMBER

H. Braun, D. Brick, A. Fridman, J-P Gerber, E. Jegham,

P. Juillot, G. Maurer, A. Michalon, C. Voltolini (Centre

de Recherches); P. Antich, R. Attendoli, E. Calligraich,

G. Cecchet, R. Dolfini, G. Fumagalli, G. Liquori, L. Mapelli,

S. Ratti and M. Tomaini (University of Pavia)

In contrast to the large amount of data accumulated in non strange baryon-baryon interactions, there is only a limited amount of information available on $\Sigma N$ interactions. As a $\Sigma p$ experiment at $240 \mathrm{GeV} / \mathrm{c}$ has already been approved with the $1^{\prime} 5^{\prime}$ we propose to complete this experiment by studying the $\Sigma^{-} n$ interactions at the same incident momentum. This will allow us to make a comparison between $\Sigma \mathrm{n}$ and $\Sigma^{\prime} \mathrm{p}$ reactions and to detect thus the isospin influence on the $\Sigma \mathrm{N}$ interactions. Furthermore, as previous bubble chamber experiments at Fermilab have shown that four constraint reactions can be studied, the use of a neutron target will give us access to four_constraint final states which cannot be obtained from the $\Sigma^{-} p$ experiment. The utilization of a deuteron target will of course complicate somewhat the data analysis. Nevertheless this is a convenient method to study the pure $I=3 / 2 \Sigma N$ isospin state as $\Sigma^{+} p$ experiments are more difficult to carry out than $\Sigma^{-} n$. In a first step we would like to have a statistics corresponding to 50,000 multiprong events, i.e., events having more than two prongs.

The 15' bubble chamber is particularly well adapted for the proposed experiment. Indeed its large size will increase the probability to observe the decays of strange particles which will be produced abundantly because of the $S=-1$ strangeness value of the initial state. Apart of this mean feature other advantages are connected with the use of the $15^{\prime}$ i.e.:

- The possibility of measuring momenta with a good accuracy

- The high probability of observing secondary interactions facilitating thus the identification of the outgoing particles.

- The high $\gamma$-ray conversion rate allowing to obtain information on $\pi^{\circ}$ and $\Sigma^{\circ}$ production.

Using a fiducial region of $2.8 \mathrm{~m}$ and assuming that one can have $5 \Sigma^{-}$/burst we will need about 57,000 pictures.

Summary prepared by A. Fridman/J. Lach, May 1976 
STUDY OF CASCADE SHOWER ORIGINATED IN JET SHOWERS

S. Dake (Kobe Univ.); Y. Nakanishi, M. Sakata and Y. Yamamoto (Konan Univ.); K. Mizutani (Saitama Univ.); T. Yuda (Univ. of Tokyo); I Ohta (Utsunomiya Univ.)

The purpose of our experiment is to observe the longitudinal development and the lateral structure of the cascade showers originated in the jet shower in emulsion chamber. The results can be compared with the pure electro-magnetic cascase shower initiated by electron, which is our other proposal \#340. If the difference between the above two kinds of shower is made clear, we can discriminate them in cosmic ray experiments. And also, we can distinsuish the nuclear events from the Bremsstrahlung events in muon experiment with emulsion chamber.

As the proposal \#434, the three type emulsion chambers had been exposed for $400 \mathrm{GeV}$ proton beam on December 8 in Fermilab. Two chambers of them are the same type which is composed of 16 nuclear emulsion plates $(9 \mathrm{~cm} \times 12 \mathrm{~cm} \times 0.8 \mathrm{~mm})$ and 12 lead plates $(9 \mathrm{~cm} \times 12 \mathrm{~cm} \times 0.5 \mathrm{~cm})$ piled up alternatively except first four emulsion plates. The remaining one is constituted 28 nuclear emulsion plates and 24 lead plates $(9 \mathrm{~cm} \times 12 \mathrm{~cm} \times 2.5 \mathrm{~cm})$. The total thickness of lead absorber is 10.5 radiation length for all chambers. The amounts of irradiation were about 200 protons/ $\mathrm{cm}^{2}$ on each detector.

Now, we are analyzing the emulsion chambers.

Summary prepared by

S. Dake, January 1976 
MEASUREMENT OF THE POLARIZATIONS OF PROMPT MUONS $A T x=0.14$ at $P_{t}=0$ and $P_{t}=1.5 \mathrm{GeV} / \mathrm{C}$

R. Adair, P. Bergey, D. Grannan, H. Kasha, R. Kellogg, M. Lauterback (Yale), L. Leipuner, R. Larsen, L. Smith (BNL), and R. Stefanski (Fermilab)

Continuing the work begun in E48 this group proposes to look at the polarization of prompt muons produced at $x=0.14$ in the forward direction and a transverse momenta near $1.5 \mathrm{GeV} / \mathrm{c}$. These proposed measurements include the kinematic region where the anomalously large production of muons has been observed. If the production process is non-parity conserving there will be a longitudinal polarization of the muons.

The polarimeter is a large block of aluminum and detectors within a low magnetic field. The muons are stopped in the aluminum and the distribution of the decay electrons is measured. In order to accomplish these measurements the polarimeter must be moved, but no additional equipment is required. The experimenters estimate that they will need 250 hours in Proton center to carry out these measurements. 
DETERMINATION OF THE POSSIBLE DI MUON CHARACTER OF THE PROMPT MUON FLUX

R.K. Adair, P.D. Bergey, D.M. Grannan, H. Kasha, R.G. Kellogg, M.J. Lauterbach (Yale Univ.); L.B. Leipuner, R.C. Larsen, L.W. Smith (Brookhaven); R.J. Stefanski (Fermilab)

We propose to measure the di-muon flux produced by the interaction of $400 \mathrm{GeV}$ protons with copper and compare this flux with the flux of single prompt muons in a manner which will determine whether the prompt muons flux is derived from muon pair production. We will measure the production of muon pairs such that each muon has a laboratory energy greater than $55 \mathrm{GeV}$ and a production angle less than 35 milliradians (or transverse momentum less than $2.0 \mathrm{GeV} / \mathrm{c}$ ). We demonstrate that we can determine the total cross section for the production of muon pairs, such that each muon has a laboratory energy greater than $55 \mathrm{GeV}$ (or $x$ greater than 0.1375), from our measurements. With the same apparatus, we will measure the total prompt muon flux such that the muon has an energy greater than $55 \mathrm{GeV}$. Using measurements made previously by us concerning the ratio of the prompt muon flux to the pion flux as a function of $x$ for small $\mathrm{P}_{t}$, together with measurements of the pion production spectrum made at various laboratories, we can correct for the energy dependence of the cross sections and then compare the total cross section for di-muon production with the total cross section for prompt muon production and then determine whether these cross sections are consistent with the view that they derive from the same source.

We will need about 75 hours of beam time using a set-up and equipment which are minor variations of the equipment used for Experiment 48. We will need one of the portacamps used in Experiment 48 moved about $16^{\prime}$. We will need 6 more duo-quad logic units from PREP. About 35 hours of the requested time will be required for data taking, about 40 for set-up and test time.

Summary prepared by

R. K. Adair, September 1975 


\section{INELASTIC CROSS SECTIONS OF NEUTRONS ON NUCLEI}

I.W. Jones, C.A. Ayre, H.R. Gustafson, M.J. Longo, T. Roberts (University of Michigan)

It is proposed to measure inelastic cross sections of neutrons on complex nuclei over the Fermilab energy range. These cross sections on light nuclei are expected to reflect the rise seen in the nucleon-nucleon cross sections whereas little or no rise is seen in the total neutron-nucleus cross section as the onset of the inelastic screening effect largely offsets the rise resulting from the nucleon-nucleon increase. The inelastic cross sections are relevant in determining the interaction mean free paths of protons and neutrons in various elements.

The experiment would be done in the $M 3$ neutral beam in the Meson Area. Target nuclei would include $\mathrm{Be}, \mathrm{C}, \mathrm{Al}, \mathrm{Fe}, \mathrm{Cu}, \mathrm{Cd}$, $\mathrm{W}, \mathrm{Pb}$ and $\mathrm{U}$. Determinations would be accurate to at least 18 . The calorimeter detector of E4 would be used along with other equipment from that experiment. A count in a scintillation counter located between the target and the calorimeter would define an inelastic interaction. All energetic reaction products would pass forward into the calorimeter and the measurement of their total energy would give the energy of the incident neutron. An event with no count in the scintillation counter would be due to an elastically scattered neutron or to one that has not interacted. A monitor would be located in the beam upstream followed by an anticoincidence counter before the target.

It is estimated that 18 cross section data can be collected in 5 energy bins in about an hour. 500 hours are requested for the experiment with control of the beam following parasitic set up and testing. 


\section{HIGH SENSITIVITY SEARCH FOR NEW STATES}

WHICH DECAY INTO MUONS

D. Garelick, P. Gauthier, M. Glaubman, J. Johnson, M. Mallary, E. Pothier, D. Potter, M. Ronan, E. von Goeler (Northeastern University)

This experiment will measure the reaction

$$
\mathrm{p}+\mathrm{Fe} \rightarrow \text { muons + anything }
$$

in order to search for new states up to $25 \mathrm{GeV}$ in mass which decay into two or more muons. A "beam dump" method will be used with a beam of $400 \mathrm{GeV}$ protons at an intensity of $\sim 10^{10}$ protons per pulse. This method uses a pencil beam, magnetized Fe and proportional chambers to determine muon angles and momenta and the effective mass of the muons detected in each event. In 300 hours of data taking, new states with $\sigma B \sim 10^{-39} \mathrm{~cm}^{2}$ per nucleon will appear as a 25 event signal.

Summary prepared by

D. Garelick, January 1976 
PROPOSAL FOR A NEW MEASUREMENT OF THE

MAGNETIC MOMENT OF THE $\Lambda^{\circ}$ HYPERON

G. Bunce, R. Handler, R. March, M. Sheaff and L. Pondrom

(Univ. of Wisconsin); $K$. Heller and $O$. Overseth (Univ. of Michigan); T. Devlin and R. Edwards (Rutgers University)

$\Lambda^{\circ}$ produced at $6 \mathrm{mrad}$ by $400 \mathrm{GeV}$ protons through the inclusive process $\mathrm{p}+\mathrm{Be}+\Lambda^{0}+$ anything have a useful average polarization $\left\langle\alpha \underline{\mathrm{P}}_{\Lambda}\right\rangle=.1$. It is proposed to exploit this polarization to measure the magnetic moment of the $\Lambda^{\circ}$ to better than 18. To do this, the angle of precession of the $\Lambda^{\circ}$ spin, $w$, will be measured in a 10-foot bending magnet placed in the neutral beam. A flux of 45,000 $\Lambda^{\circ}$ /hour can be obtained, giving a data sample of $4 \times 10^{6} \Lambda^{\circ}$ in 100 hours. The error in the precession angle for $\omega=\pi / 4$ is $\delta \omega / \omega=13 / \sqrt{\mathrm{N}}=6 \times 10^{-3}$ for $\mathrm{N}=4 \times 10^{6}$. A run of 160 hours is requested.

Summary prepared by

G. Bunce, November 1975 


\section{PROPOSAL TO STUDY $\Lambda^{\circ}$ POLARIZATION IN THE INCLUSIVE REACTION $p+p \rightarrow \Lambda^{\circ}+$ ANYTHING WITH A LIQUID HYDROGEN TARGET}

G. Bunce, L. Pondrom, R. March, R. Handler and M. Sheaff (Univ. of Wisconsin); T. Devlin and R. Edwards (Rutgers Univ.); $K$. Heller and $O$. Overseth (Univ. of Michigan)

It is proposed that a $50 \mathrm{~cm}$ liquid hydrogen flask be installed at the hyperon production target to study $\Lambda^{\circ}$ polarization in pF collisions with $400 \mathrm{GeV}$ protons. Measurements of $\underline{p}_{\Lambda}\left(x, p_{\perp}\right)$ have already been made in $p+B e$ collisions with sufficient accuracy to establish the effect, but to learn more about the physical process which causes the polarization it is necessary to use a proton target and to extend the range of $\mathrm{p}_{1}$ studied. A 150 hour run is requested.

Summary prepared by

L. Pondrom, November 1975 
STUDY OF NUCLEAR FRAGMENT EMISSION IN PROTON HEAVY NUCLEUS COLLISION FROM 10 TO $500 \mathrm{GeV} / \mathrm{C}$

E. Turkot, J. McCrary (Fermilab): J.A. Gaidos, L.J. Gutay, R.P. Scharenberg, R.B. Willman, T.V. Ragland, P. Leong (Purdue University)

We propose to measure the double differential cross section
$\frac{d^{2} \sigma}{d \Omega d E}$ of nuclear fragments $(2 \leq z \leq 16)$, for a given incident momentum, produced by/heavy nucleus $(20 \leq z \leq 80)$ collisions in the 10 to $500 \mathrm{GeV} / \mathrm{c}$ momentum range. Recent éxper̄imental results and theoretical speculations suggest colletive nuclear motion is initiated by the passage of a fast proton through a heavy nucleus. The novel feature of this experiment is the determination of the energy dependence of the excitation mechanism. Because nuclear fragments are best studied from thin targets, one needs a very high beam flux to obtain sufficient event rates. These considerations make the Internal Target Area with a heavy gas jet target the ideal place to initiate our experimental program. The detection system will consist of four conventional $\Delta \mathrm{E}-\mathrm{E}$ semiconductor telescopes.

If the measured angular distributions exhibit features compatible with the nuclear shock wave phenomena or if the energy dependence of the excitation mechanism for the emission of nuclear fragments indicates unusual structure, we will propose to incorporate a time-of-flight capability into the apparatus and search for abnormally cense nuclear fragments whose existence could be indicated by anomalous $z / M$ ratios.

Three to four calendar months of set up and testing time are requested in the Internal Target Area followed by a three month period for data taking ( 400 hours) at $-50 \%$ duty cycle.

Summary prepared by

F. Turkot/J. Lach, November 1975 
A PROPOSAL FOR CONTINUED STUDIES OF

HADRON INDUCED $\mu$-PAIRS IN A LARGE ACCEPTANCE SPECTROMETER

K.J. Anderson, G.G. Henry, K.T. McDonald, J.E. Pilcher, E.I. Rosenberg (Univ. of Chicago); J.G. Branson, G.H. Sanders, A.J.S. Smith, J.J. Thaler (Princeton)

It is proposed to complete the study of hadron induced $\mu$-pairs as set out in Experiment \#331 using the cyclotron spectrometer. For reasons of scheduling and to minimize interference with Experiment \#98, the running of Experiment \#331 has been restricted to nuclear targets and a positive beam almost an order of magnitude less intense than the spectrometer can handle. The experimenters wish to extend their studies to hydrogen and deuterium targets and to use a higher quality hadron beam into the cyclotron spectrometer.

The physics goals of the experimert remain the same; namely, to study the hadron production of $\mu$-pairs in a large acceptance detector with a minimum of detection biases. They will study the $\mu$-pair production cross section as a function of $\mathrm{x}_{\mathrm{F}}$, $\mathrm{pt}$ and $M_{\mu \mu}$ for incident pions, protons, and kaons. They will also measure the cecay angular distributior of the $\mu$-pair state.

of special interest are the continuum $\mu$-pair characteristics. The experiment claims to be a unique position to study the continuum production by protons, as well as pions of both charges, interacting with free nucleons. These data will provide important constraints to any parton model of the hadrons.

The experiment would use a new one meter long cryogenic target and a beam flux of $10^{7}$ particles per pulse. Such a beam intensity could be obtained during normal neutrino running with the horn system by adding a third split to the primary proton beam and targeting in Enclosure 100. Another possibility is a special neutrino decay pipe triplet recently suggested. It is estimated that this experiment will require about 400 hours of beam time.

Summary prepared by J.E. Pilcher/A.F. Greene, November 1975 
A SPECIAL REQUEST FOR HIGH-PRIORITY RUNNING TO MEASURE HIGH-MASS MUON PAIRS

K.J. Anderson, G.G. Henry, K.T. McDonald, J.E. Pilcher, E.I. Rosenberg (Univ. of Chicago); J.G. Bronson, G.H. Sanders, A.J.S. Smith, J.J. Thaler (Princeton)

A request is made for 400 hours or 4 weeks of high priority running in the Neutrino Area to continue work begun in Experiment \#331 using the cyclotron spectrometer. During this time use would be made of the quadrupole triplet focusing system to provide positive and negative hadron beams of $225-300 \mathrm{GeV}$ at irtensities up to $10^{7}$ particles per pulse. It is asked that this running period occur in Feb.-March, 1976 and that $1-2 \times 1012$ protons per pulse at $400 \mathrm{GeV}$ be provided.

The new results which would be obtained from this running are:

1. High-sensitivity search for new particles. A sensitivity of about 1 event per $10^{-36} \mathrm{~cm}^{2} /$ nucleon should be possible up to dimuon masses of $15 \mathrm{GeV}$.

2. Tests of production mechanisms of continuum muons. Using positive and negative beams with an isotopicallysymmetric target material, such as carbon, it is possible to test features of various parton models.

3. Detailed study and comparison of continuum and $\psi(J)$ production. Measurement of the density matrix for the $\psi(\mathrm{J})$ and comparison with the continuum should provide information on the mechanism for its production.

4. Other studies will include study of like-sign dimuon events, events with more than two muons, and kaoninduced muon pairs.

Summary prepared by A.J.S. Smith/A.F. Greene, November 1975 
THE DIRECT DETERMINIATION OF KAON AND PION FORM FACTORS

Fermi National Accelerator Laboratory, Yale University C. Ankenbrandt (Spokesperson)

\begin{abstract}
$\mathrm{F}\left(\mathrm{Q}^{2}\right)$ It is proposed to measure the pion and kaon form factors, Data would be taken simultaneously for both particles.
\end{abstract}

The experiment would use the equipment of $\mathrm{E} 69$ with modifications. The $52 \mathrm{~cm} \mathrm{LH} 2$ target is surrounded by veto counters with a small hole in the forward direction. Proportional wire chambers before and after the target measure the angles of the incident particle and both scattered particles to $0.03 \mathrm{mr}$. A momentum spectrometer further downstream would consist of one of the $M 6 \mathrm{~W}$ bending magnets (the second being removed) and proportional wire chambers. The resultant momentum resolution would be $0.22 \%$ at $200 \mathrm{GeV} / \mathrm{c}$. Following this is a lead-scintillator sandwich shower counter to measure electrons, and a hacron colorimeter to measure muons, that pass through the spectrometer. The more forward going particle of the two scattering products, meson or electron, will pass through the spectrometer system. In the case of Ke scattering this will always be the kaon. For te scattering it will be the pion up to $Q^{2} \approx 1 / 2 Q_{\text {max }}{ }^{2}$ and, for $\pi^{-} e$ scattering, the electron above that. Since all angles are known as well as the momenta of the incident particle and one of the outgoing particles, a $3 \mathrm{C}$ fit will be obtained for each elastic scattering event.

For Ke scattering at $200 \mathrm{GeV} / \mathrm{c}$, for example, the overall range of acceptance is $0.014 \approx \mathrm{Q}^{2} \leqslant 0.093(\mathrm{GeV} / \mathrm{c})^{2}$. The point-like cross section over this range is $11 \mu \mathrm{b}$; for the region above $\mathrm{Q}^{2}=0.05(\mathrm{GeV} / \mathrm{c})^{2}$ it is $0.67 \mathrm{\mu b}$. Geometrical acceptance is essentially $100 \%$ over the range except at $Q^{2} \approx 1 / 2 Q_{\max }{ }^{2}$. Event rates assume a total of $10^{6}$ particles/spill at $8 \mathrm{sec}$. intervals and an overall safety factor of two. At $-200 \mathrm{GeV} / \mathrm{C}, 3.2 \times 10^{4}$ Ke events would be measured over the full range of $Q^{2} \cdot 2.4 \times 10^{5}$ Te events would also be obtained. The triggering system consists of fast logic and analog circuitry to yield 300 triggers per spill. The experiment would yield $R_{K}$ to an accuracy of $6 \%$ and $R_{\pi}$ to $2-38$. A total of 800 hours is requested to run at $-200,+200$ and $-150 \mathrm{GeV} / \mathrm{c}$. New apparatus required would be ready to install in July 1976 . 


$-280-\quad \underline{30-\text { Inch }} \underline{\overline{\mathrm{p}}}-\mathrm{p}$ e $150 \# 447$

A pRoposal to STUdy $\overline{\mathrm{p}}-\mathrm{p}$ INTERACTiONS AT $150 \mathrm{GeV} / \mathrm{c}$

Brown Univ., Fermilab, IIT, Univ. of Illinois, MIT, Yale Univ., Univ. of strasbourg, Univ. of Tel-Aviv, Weizmann Inst. R. Hulsizer (Spokesperson)

We propose an exposure of 0.6 events/ $\mu \mathrm{b}$ for antiprotons as close as possible to $150 \mathrm{GeV} / \mathrm{c}$ in the FNAL 30-inch Bubble ChamberProportional wire Chamber Hybrid Spectrometer System. We request these exposures as soon as suitably enriched beams of antiprotons are available to the spectrometer system. For a beam with antiprotons consisting of a fraction $f$ of the total beam and eight beam tracks per picture, an exposure of $62,500 / f$ pictures will yield 25,000 events for the study.

An integral part of the proposal is an effort to seek improved methods of obtaining antiproton-enriched beams, and to complete the forward gamma detector now under construction.

The purpose of this study is to gain some insight into the consequences of the difference between the valence quark makeup of protons and antiprotons. This will be accomplished by comparing the interactions proposed here with the p-p interactions being studied at the same energy in E-299. In particular, the availability of the forward gamma detector will enable us to compare the $\rho^{+}, \rho^{-}$, and $\rho^{0}$ production in the central region in the two processes. The availability of an external particle identifier would considerably expand the understanding we could gain of the differences between pp and other interactions. 
PROPOSAL FOR THE

INVESTIGATION OF VIRTUAL PHOTOABSORPTION BY NUCLEAR MATTER

W.A. Loomis, F.M. Pipkin, A.L. Sessoms, R. Wilson (Harvard Univ.); W. Francis, T.W. Kirk (Univ. of Illinois); T. Quirk, W.S.C. Williams (Oxford Univ.); H.L. Anderson (Univ. of Chicago)

The behavior of muon-nucleus scattering presents an apparent paradox which can possibly be resolved by various forms of Generalized Vector Dominance. The experimental investigation of the paradox requires that data be taken at very large values of the scaling parameter

$$
\omega=\frac{2 M v}{q^{2}}
$$

The Muon Scattering Facility is equipped for these studies, and it is proposed to carry out the investigation.

It long seemed obvious that nuclei should be transparent to $\gamma$ rays of any energy, since the total cross section for $\gamma$ rays on protons - $125 \mathrm{\mu b}$-- is much less than the "geometric" cross section of a proton -- $40 \mathrm{mb}$. Yet measurements made with real photons with energies greater than a few GeV show that $\sigma_{T}(\gamma A) / A \sigma_{T}(\gamma N)$ is much less than 1 indicating opacity. The opacity is assumed to arise from the existence of the vector mesons $\rho^{\circ} \omega^{\circ} \phi^{\circ}$, (and now presumably $\rho^{\prime}, \psi, \psi^{\prime}$ also) which have the same quantum numbers as the photon, and strong interactions. At a high encugh entrgy, the mass difference is negligible in the sense that the photon and a rho meson will stay in phase over a distance the size of a nucleus, then the photon can appear to have the strong interactions of the rho meson in spite of the low total cross section. The critical parameter which separates the two variables -- transparency and opacity -- is the ratio $\mathrm{k} / \mathrm{m}_{\mathrm{v}}^{2} \lambda$ where $k$ is the $\gamma$ ray energy, $\lambda$ is the absorption length of the vector meson in nuclear matter and $m_{\mathrm{V}}$ is the mass of the vector meson. When this parameter is large compared with unity, nuclei will show shadowing; when small, transparency. Theory suggests that the shadowing effect should disappear as $\omega$ decreases.

More proportional chambers will be added to the E98 apparatus including some in the middle of the magnet at some distance from the target. It is proposed to run the experiment with three nuclear targets: beryllium, copper and lead. These data will be combined with the deuterium data of E98. In order to see the shadowing effect clearly, and in particular to see many rho muons, the proponents would like to have about 20,000 events on each target ( $3 \%$ statistics in each of $10 \mathrm{w}$ bins). A beam of $5 \times 10^{5}$ per pulse is requested for 285 hours (or 8 x 1010 muons at 150 $\mathrm{GeV} / \mathrm{C})$ with the best possible duty cycle. The experiment could be performed anytime after the completion of E398. 


\section{A Proposal to Measure Coulomb and \\ Diffractive Production of $K^{\star}, \bar{K}^{\star}$}

M. A. Abolins, B. Dodge, J. A. J. Matthews, R. A. Sidwell

Michigan State University

H. R. Barton, Jr., N. W. Reay, N. R. Stanton

The Ohio State University

\section{K. W. Edwards \\ Carleton University}

We will measure the production of $K^{\star} 890, \bar{K}^{\star} 890$ using the $K_{L}^{\circ}$ component of a hardened $M-3$ beam incident on a variety of targets from $C$ to $U$. Using the Primakoff effect this will permit extraction of the radiative width of the $K^{*}(390)$ and possibly $K^{\star}(7420)$. These measurements yield important tests of quark and $\mathrm{SU}_{3}$ models. Measurements will cover the range $0 \leq \mathrm{t} \leq .1 \mathrm{GeV} / \mathrm{c}^{2}$ and $30 \leq p \leq 140 \mathrm{GeV} / c$. The apparatus will be a vee spectrometer with point to parallel focusing using two Cerenkov counters to identify decay products.

We request of Fermilab a BMI09 magnet shimmed to $10^{\prime \prime}$, absorbing material LiH or (Be) $2.4 \mathrm{~m}(1.1 \mathrm{~m})$ remotely movable, a Bison PDP $11 / 45$ system, $\$ 85,000$ of PREP equipment, a $6 "$ I.D. by $5 \mathrm{~m}$ vacuum decay pipe. Initial running time requested is 600 hours with $3 \times 10^{12} 400 \mathrm{GeV}$ protons on the meson target. 
D. Caldwell, J. Cumalat, A. Eisner, R. Morrison, F. Murphy, S. Yellin (Univ. of Calif., Santa Barbara); P. Davis, R. Egloff, G. Luste, J. Prentice (Univ. of Toronto); T. Nash (Fermilab)

It is proposed to continue a study of $\psi(\mathrm{J})$ and $\psi^{\prime}$ photoproduction on deuterium that was begun during the summer of 1975 as a test of the apparatus and tagging system for Experiment \#25A. Interest is shown by the experimenters in a continuation of this work to include a running period of 400-800 hours with $3 \times 10^{12}$ protons/pulse at $400 \mathrm{GeV}$. With an event rate under these conditions of $1.3 \psi(\mathrm{J})$ events per hour it is expected to obtain a minimum of $500 \psi(\mathrm{J})$ events and possibly $20 \psi^{\prime}$ events.

The specific goals of this experiment are:

1. Measurement of the photoproduction cross section of $\psi(\mathrm{J})$ on deuterium as a function of energy from about $35 \mathrm{GeV}$ to $130 \mathrm{GeV}$.

2. Measurement of the elastic part of the $\psi(J)$ cross section as a function of energy and of momentum transfer.

3. Determination of the $\psi^{\prime}$ photoproduction cross section.

4. Search for new photoproduced high mass states which decay into electrons, photons, and $\pi^{\circ}$ 's including heavy leptons possibly.

The experiment would use equipment currently set up in the Tagged Photon Laboratory. The necessary apparatus for these measurements includes a deuterium target surrounded by twelve "barrel stave" recoil detectors, multi-wire proportional chambers used to measure electrons from, for example, the decay $\psi(3.1)+e^{+} e^{-}$. A lead-glass array for identifying showers from electrons or $\pi^{\circ}$ 's and a hadron calorimeter will then follow. Most all of this equipment is required for use in the already approved Experiment \#25A where measurements will be made of the photon total cross section into hadrons. 
Bari Univ. (Italy): C. DeMarzo, L. Guerriero, P. LaVopa, G. Magg1, F. Posa, G. Selvaggi, P. Spinelli, F. Waldner; Brown Univ.: D. Cutts, R. Dulude, R. Lanou, Jr., J. Mass1mo; FNAL: A. Brenner, L. Carey, J. Elias, G. Mikenterg; MIT: W. Altkenhead, B. Barton, G. Brandenburg, W. Busza, J. Friedman, P. Garbinclus, H. Tendal1, B. Nelson, L. Rosenson, W. Toy, R. Verdier, C. Young; HEPL (Warsaw, Poland): I.P. Z1elinski.

It is proposed to study the A-dependence of Inclusive reactlons over a wide range of kinematics using the $M-6$ single arm spectrometer facility along with a detector for $\pi^{0} / s$ produced at large $\mathrm{p}_{\mathrm{t}}$. The multiplicity detector of experiment E118A will be used to obtain the associated charged multiplicity and pseudo-rapidity distribution assoclated with each event.

The principal objective is to explore these processes over a wide range of $A, x$, and $p_{t}$. In particular, we w1ll run at $175 \mathrm{GeV} / \mathrm{c} w 1 t h \pi^{+}, \mathrm{K}^{+}$, and $\mathrm{p}$, incident on $\mathrm{H}_{2}, \mathrm{Be}, \mathrm{Al}, \mathrm{Cu}, \mathrm{Sn}$, and $\mathrm{Pb}$. We w111 obtaln data on the Adependence of the inclusive distributions of $\pi^{+}, \mathrm{k}^{+}$, and $\mathrm{p}^{+}$produced from these targets for values of $x$ from 0.1 to 0.95 and from $\mathrm{p}_{t} \approx 0$ to $\mathrm{p}_{\mathrm{t}} t 1.25$. Simultaneously data will be obtained for the inclustve $\pi^{\circ}$ distribution for $1.0 \lesssim \mathrm{p}_{\mathrm{t}} \lesssim 4.0$. Statist 1 cal errors wil1 range from about $2 \%$ at the smallest transverse momenta to about $15 \%$ at the largest transverse momenta. The running time needed for this program is estimated to be 500 hours plus 100 hours for setup and testing.

The $\pi^{\circ}$ detector will consist of an array of lead-glass preceded by a shower bodoscope of lead-scintillator construction. It will subtend 20 mster. between 70 and $210 \mathrm{mr}$. In the $1 \mathrm{ab}$. The transverse momentum of the $\pi^{\circ}$ will be determined to about $10 \%$. Cronin et al. and Lederman et al. have shown that the A-dependence of the inclusive $\pi^{\circ}$ cross section increases from $\mathrm{A}^{2}$ to $A$ going from $\mathrm{p}_{\mathrm{t}} \nsucc 0$ to $\mathrm{p}_{\mathrm{t}} \AA 4$, a result which is totally unexpected from current models. We will be able to measure the correlation of this phenomenon with the associated mult1plicity and Incldent particle type.

This measurement will provide extensive information on leading particle effects in inclusive production from nuclei. All current models predict a weak A-dependence for the leading particle. In addition, spectrometer events with $x<0.3$ should show the onset of the strongly A-dependent part of the rapidity distribution. The posttion of this feature as a function of energy is a crucial test for some models. The substantial particle 1dent1fication power of the SAS facility will allow simultaneous measurement of all combinations of $\pi^{+}, \mathrm{K}^{+}$, and $\mathrm{p}^{+}$in the beam and in the spectrotreter. In this way, the dependence of the hadron-nucleus cross sections and multiplicities on the identity of the incldent and produced particle may be compared. 


\section{PROPOSAL FOR A STREAMER CHAMBER FACILITY TO STUDY $\psi$ PRODUCTION}

T. Burnett, V. Cook. S. Csorna, D. Holmgren, H.J. Lubatti,

K. Moriyasu (Univ. of Washington), J. Bartke, A. Eskreys,

P. Malecki, K. Zalewski (Inst. of Nuclear Physics, Krakow)

From Fermilab experiments it is known that the $\psi$ (3.1) appears to be produced centrally at small $x$ in hadronic reactions, but almost nothing else is known about the reaction mechanism. It has been proposed that the $\psi$ mesons are evidence for the existence of a new quantum number (charm) which should result in new multiplets of charmed resonances. The failure of the firstround of searches to find these resonances clearly indicates the importance of a detailed study of the $\psi$ production mechansim at Fermilab energies with both pion and proton beams.

It is proposed to study the reaction $\pi^{-}(\mathrm{p})+\mathrm{p}+\psi+$ anything using a streamer chamber with a hydrogen target. The streamer chamber provides the detection efficiency necessary for studying the charged particles produced in association with a $\psi$ meson. The trigger will be provided by two muons whose invariant mass is greater than $\sim 1 \mathrm{GeV}$. In order to perform this experiment it is proposed to construct a new streamer chamber. This new chamber and the magnet into which it would be placed would provide a useful detector for many experiments.

The apparatus consists of a $2 \times 1 \times 0.6 \mathrm{~m}^{3}$ streamer chamber inside a new superconducting magnet of $40 \mathrm{kilogauss}$ meters. A magnetized iron absorber will be placed downstream of the streamer chamber. The trigger for the experiment will be muons from the decay $\psi(3.1) \rightarrow \mu^{+} \mu^{-}$, with the two muons detected in hodoscope arrays and identified with fast electronics.

The experiment would utilize a charged hadron beam with energies greater than $100 \mathrm{GeV}$ and intensities of $10^{6}$ particles per pulse. A downstream location in the Ml beam in the Meson Area seems preferable. Estimates of required running time to obtain $2,000 \psi(3.1)$ events are one month or about 400 hours. 


\section{A PROPOSAL TO CONTINUE DIRECT MUON PRODUCTION IN THE FORWARD DIRECTION}

D. Buchholz (Northwestern), H.J. Frisch, M.J. Shochet (Univ. of Chicago), R.P. Johnson (Fermilab), R.L. Sumner (Princeton), O. Fackler, S.L. Segler (Rockefeller)

The origin of direct lepton production is as yet not understood. The phenomenon was first discovered at large values of $P_{\perp}\left(P_{\perp}>1.5 \mathrm{GeV} / \mathrm{c}\right)$, a kinematic region which itself is not well understood. Questions of the $s, x$, and $p_{1}$ dependence of the direct muon to pion ratio still exist almost two years after the publication of the first Fermilab results. Recently these experimenters have completed the first measurements of the direct muon production at very low $p_{1}$, i.e. $p_{1} \leq 0.4 \mathrm{GeV} / \mathrm{c}$. These measurements were made during a short period of data-taking for Experiment \#335 at Fermilab in the Ml beam of the Meson Area.

A brief summary of the results and their significance is presented here:

1. In $300 \mathrm{GeV}$ proton-Uranium collisions the ratio of direct muons to pions is larger at $p_{\perp} \leq 0.4 \mathrm{GeV}$ and $x=0.3$ than for $p_{\perp}>$ $1.5 \mathrm{GeV}$ at $\mathrm{x}=0$.

2. The ratio $\mu^{-} / \pi^{-}$is smaller at $x=0.5$ than at $x=0.3 ; i . e$. , the direct muon production is steeper in $\mathbf{x}$ than the pion production.

3. Equal numbers of direct $\mu^{+}$and $\mu^{-}$are created, although far more $\pi^{+}$are created than $\pi^{-}$. This is consistent, for example, with the direct muons (or their parents) being created in pairs.

These results seem to rule out heavy vector mesons as the source of direct leptons. They are consistent, however, with the many-body semi-leptonic decay of a heavy particle, or with either lighter vector parents or continum creation of the muons.

It is proposed to improve and extend these measurements in either the MI or M2 beam operated as a normal charged secondary beam at $200 \mathrm{GeV} / \mathrm{c}$ with $5 \times 1012$ particles/pulse. The target and forward spectrometer would be moved to the Meson detector building. The apparatus will contain an upstream Cerenkov counter to identify beam muons, a variable density target, and a downstream absorber. The downstream spectrometer will have two EPB dipoles with multiwire proportional chambers on either side. A steel muon identifier is placed at the end of the apparatus.

The request for running time includes 200 hours for tuning and background studies and 400 hours for data-taking. 
EXPERIMENT TO MEASURE DEEP INELASTIC ELECTRON SCATTERING ON HYDROGEN AND DEUTERIUM WITH SEPARATION OF $\cup W_{2}$ AND $W_{1}$ NUCLEON STRUCTURE FUNCTIONS, AT THE HIGHEST FERMILAB ENERGIES AND $Q^{2}$ REGIONS

G. Conger, J. Edighoffer, A. Grigorian, Z.G.T. Guiragossiän, R. Hofstadter, T.P. McPharlin, M.R. Yearian (Stanford Univ. HEPL), B.C. Cox, J. Peoples Jr. (Fermilab)

The physics objective of the experiment is to measure inclusiv. deep inelastic electron-nucleon scattering cross sections by $\mathrm{e}^{-}$and $\mathrm{e}^{+}$beam bombardment on $24 "$ long $\mathrm{LH}_{2} / \mathrm{LD}_{2}$ targets at $150 \mathrm{GeV}\left(5 \times 10^{8}\right.$ $\left.\mathrm{e}^{ \pm} / \mathrm{pulse}\right), 175 \mathrm{GeV}\left(3.6 \times 10^{8} \mathrm{e}^{\ddagger} / \mathrm{pulse}\right)$ and $250 \mathrm{GeV}\left(1 \times 10^{8} \mathrm{e}^{ \pm} / \mathrm{pulse}\right.$ beam energies. Cross sections will be measured in the range of $\mathrm{Q}^{2}=0.16--160(\mathrm{GeV} / \mathrm{C})^{2}$ and in the range of hadronic mass squared

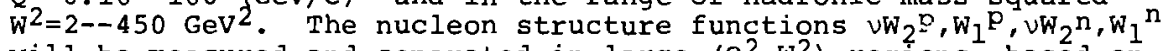
will be measured and separated in large $\left(Q^{2}, W^{2}\right)$ regions, based on values of the polarization parameter $E$ spanned by the three beam energies. The influence of any two-photon-exchange effects will be identified by measuring the asymmetry in $\sigma\left(e^{+} p\right)-\sigma\left(e^{-} p\right)$ as a function of $\mathrm{Q}^{2}$. The scaling behavior and variation of the four nucleon structure functions will be determined by direct measurements and separation in unexplored Fermilab kinematical regions. Unresolved differences between the scaling behavior of proton and neutron form factors will be elucidated.

Based on the enhanced synchrotron radiation compensated beam line tuning technique, the proton-West pion beam will be used to produce at no additional cost a clean electron beam. The experimental apparatus of E-192 at the Proton-West Experimental Hall will be used with addition of an electron arm and a minor repositioning of spectrometer arms I and II, designed to intercept with optimum acceptance scattered electrons in a large $\left(Q^{2}, W^{2}\right)$ domain, thereby obtaining excellent information on the tracking and identification of these electrons with powerful resolution in the variables $Q^{2}, w^{2}$ and $\omega^{\prime}=1+w^{2} / Q^{2}$. The experiment has a built-in self-calibration scheme, whereby ali target-associated and beam-associated backgroun occuring in a limited $\left(Q^{2}, W^{2}\right)$ domain are subtracted by simple experimental calibration runs. There are no event rate, data rate, or background rate limitations in the experiment. Requested total running time, spread over three beam energies is $1500 \mathrm{hr}$ : $e^{-p} 450 \mathrm{hr}, e^{-d} 450 \mathrm{hr}, e^{+} p 300 \mathrm{hr}$, all experimental calibrations $300 \mathrm{hr}$.

Since the changeover both in beam and apparatus between E-192 and \#454 can be made quickly, the experimenters propose to run this experiment and E-192 in interleaved periods of running time, so that they can perform quick, efficient comparisons of results, probing via respective experiments both space-like and time-like $\mathrm{Q}^{2}$ regions at the highest and as yet unexplored kinematical domains. 
R. J. Cence, F. A. Harris, S. I. Parker, M. W. Peters, V. Z. Peterson,

v. J. Stenger (Hawaii); A. Barbaro-Galtieri, P. H. Eberhard, G. R. Lynch, J. P. Marriner, and M. L. Stevenson (LBL)

Improved knowledge of dilepton (especially dimuon) production by neutrinos would become available using the combined advantages of the 15-foot bubble chamber filled with neon-hydrogen ( $30 \%$ atomic fraction), the new horn dichromatic beam, and the proposed improved EMI. EMI selection of dimuon events ( 100 dimuons per $200 \mathrm{~K} p 1 \mathrm{x}$ ) does not require f1lm scanning but the selected bubble-chamber photographs permit detailed event analysis. Knowledge of neutrino energy should reduce ambiguities.

other dilepton (e.g., e- $\mu$ ) events would also be sought and should occur in yields comparable to $\mu-\mu$ but will require a complete film scan.

Summary prepared by

v. Z. Peterson, March 30, 1976 


\section{MEASUREMENT OF THE KAON FORM FACTOR}

E. Dally, C. May, J. Hauptmann, D. Stork (UCLA); I. Ioan, T. Nigmanov, E. Tsyganov, A. Vodopianov (Dubna); T. Tompkins, T. Toohig, A. Wehmann (Fermilab); J. Poirier, C. Rey, R. Wojslaw (Univ. of Notre Dame); P. Shepard (Univ. of Pittsburgh)

The objective of this experiment is to measure the charged kaon electromagnetic form factor in the spacelike region of momentum transfer by direct observation of kaons scattered from a kaon beam by stationary electrons in a liquid hydrogen target.

A determination of the kaon radius is possibly only by such a direct elastic scattering process. The data will be taken with a $250 \mathrm{GeV}$ beam in which the beam kaons are selected by differential Cerenkov counters. Both the kaon and electron will be detected with nearly 1008 efficiency for momentum transfers from 0.03 to $0.13(\mathrm{GeV} / \mathrm{C})^{2}$. With 500 hours approved for testing and data taking, we anticipate $6000 \mathrm{~K}$-e scatters. Systematic errors can be held to $1:$ and a precision of 0.04 (Fermi) ${ }^{2}$ for the determination of the mean square kaon radius can be obtained.

The apparatus is the narrow-angle, high resolution spectrometer in the Ml West beam line used for the pion form factor experiment $\mathrm{E}-216$. The resolution will be upgraded by the use of drift chambers. Trie sfark chambers behind the magnets will be replaced by proportional wire chambers. The trajectory of the incident kaon entering a $50 \mathrm{~cm}$ long liquid hyarogen target and the scattering angles and momenta of the recoiling $k-e$ pairs will be measured by a combination of drift chambers and proportional wire chambers with the aid of two bending magnets (two BMl09's with 8" $x 24$ " aperature and $70 \mathrm{~kg}-\mathrm{m}$ fielc integral). Icientification of $\mathrm{K}-\mathrm{e}$ scatters and momentum transfer determination will be carried out through $\mathrm{kinematic}$ analysis of the recoiling particles made possibly by the high angular precision of the apparatus. A redunaancy check on the electron is suppiiec ty an electron identifier incorporating total absorption $\mathrm{Pb}-\mathrm{glass}$ shower counters. Any $\mu-e$ events are rejected in a muon filter.

Efforts will be made to achieve a low kaon trigger rate. If these efforts are successful, pion-electron scattering data will be taken in order to provide a direct comparison of the pion and kaon form factors consistent with the primary goal of the experiment. 
SEARCH FOR $\mu$ 's, KO's OR CHARACTERISTIC HADRON SIGNATURES AND MASSES IN COINCIDENCE WITH SINGLE DIRECTLY PRODUCED ELECTRONS

\author{
MIT, Brown University, Bori University \\ G. Brandenberg (Spokesman)
}

It is requested that consideration be given to tests which would eventually lead to a formal proposal for the cyclotron spectrometer in the muon/hadron beam of the Neutrino Area. The experiment itself would be a search for events containing a directly produced charged lepton (e or $\mu$ ) with the expectation of seeing the topology, character, and energy distribution in these events. The objective in this is to ensure that the events contain a rich sample of new particles decaying to leptons. Included in this sample are also production of pairs of leptons, such as $e^{+} e^{-}, \mu^{+} \mu^{-}$or $e \mu$.

At this time the experimenters are requesting approval of tests related to the rejection of Dalitz pair electrons, a large background to the reactions of interest. These tests can be described as follows:

1. Study of the ability to follow tracks measured only within the cyclotron magnet and finding a common vertex. This would require moving the presently existing $1 \mathrm{~m} \times 1 \mathrm{~m}$ proportional chambers to new locations inside the magnet.

2. Measurement of the detection efficiency and unambiguous spatial measurement of photons in magnetostrictive spark chambers. These chambers would have lead sheets interleaved between them.

To carry out these tests the experimenters request use of the cyclotron spectrometer and permission to reconfigure portions of the apparatus. Actual running will require 100 hours with a $100 \mathrm{GeV}$ pion beam incident on a solid target. 


\section{PHOTOPRODUCTION EXPEPIMENT AT FERMILAB}

B. Knapp, $h^{T}$. Lee, P. Sokolsky, W. Wisniewski (Colunbia Univ.);

G. Glading, R. Messrer, T. O'Halloran, A. Wattenberg (Univ. of Illinois); M. Binkley, I. Gaines, R. Oxr, J. Peoples (Fermilab)

We propose to continue and extend the stucy of photoproduction of massive $1^{-}$objects and the photoproduction of pairs of new particles with much improved detector. The main improvements are: 1) the geometric acceptance of the detectors is increased from: $\sim \pm 30 \mathrm{mrad}$ to $\sim \pm 150 \mathrm{mrad}$ thereby making it possible to momertum analyze charged particles produced at large angles $(\theta>60 \mathrm{mr}) ; 2)$ momentum resolution of high energy tracks has been improved leading to improved mass resolution through the use of drift chambers and a stronger magnetic field; 3) $\gamma$ ray directions as well as $\gamma$ ray energies are measured making it possible to measure the mass states of $\pi^{\circ}, \eta^{\circ}$, and $\left.\gamma^{\prime} s ; 4\right)$ the charged particles are separated into $\pi^{-1} / \pi^{ \pm} / p^{ \pm}$in certain momentum ranges by gas Cerenkov counters.

The primary objectives of the proposed experiment are 1) to study the interaction dynamics of the known massive vector mesons, $\psi^{\prime} \psi^{\prime}$, and $\psi^{\prime \prime}$, and to search for new massive vector mesons; and 2) to search for the photaproduction of heavy lepton pairs. We also plan to improve significantly our previous search for charmed particle pairs.

We request the use of the broad band photon bearr with the additional steel shielding in EE-4 as requested by $E-401$. and $5 \times 10^{12}$ protons. We request 300 hours of test time to debug the detector and 600 hours of time for data taking. 
Search for New Phenomena Associated with

High Enegy Neutrinos Using the Quadrupole Triplet Beam

U. Camerini, D. Cline, W. F. Fry, J. von Krogh, R. Loveless,

J. Mapp, R. March, D. D. Reeder(Univ. of Wisconsin);

D. Haidt, H. Wachsmuth (CERN)

It is proposed to take one million pictures of high energy neutrinos in an approximately $20 \%$ mixture of neon and helium using the Fermilab 15-foot bubble chamber exposed to the quadrupole triplet focussed neutrino beam with the external muon identifier. Experience in Experiment \#28A has shown that the capability of recording and analyzing high multiplicity, multiple gamma conversions, secondary neutron interactions, and high energy electrons makes this instrument uniquely useful in searching for new phenomena in neutrino interactions.

The principle advantage in using the quadrupole triplet beam is that a greater proportion of the events occur at high energy (i.e. $>35 \mathrm{GeV}$ ) allowing a fast and more efficient analysis of the characteristics at high energy. The results of the counter neutrino experiment indicate that new phenomena are very likely to occur at high energy. A $20 \%$ neon/hydrogen mixture makes possible the detection of high energy electrons from the primary vertex. For a substantially higher density of neon, this detection efficiency will be much worse due to a confusion of the primary with secondary vertices from interactions and radiation losses.

The main emphasis of the analysis of this experiment will be on the following:

1) Neutrino interactions producing $\mu^{-} \mathrm{e}^{+} \mathrm{K}^{0}$ in the final state. There is a need for more events like those seen in Experiment \#28A in order to understand the nature of the phenomena producing these particles. There is also indication from the earlier exposure that this production may be energy dependent. Thus use of the quadrupole triplet beam will help this study.

2) Study of neutrino interactions with two muons in the final state. Searches will also be made for strange particles associated with these events.

3) Search for new phenomena associated with high energy neutrino interactions. This may include a final state $\mu$ e with multiple strange particles or $\mu^{-} e^{+} e^{-}$events where the $e^{-} e^{+}$ are not associated with electromagnetic phenomena. Other possibilities are neutrino interactions with a final state $e^{+} e^{-}$ or neutral current interactions producing a final state $e^{+} K^{0}$.

The exposure could be made with a horn-focussed neutrino beam. However, there are advantages to using the quadrupole triplet system, related particularly to studies of energy dependence and use of the EMI to identify final state muons. It would be acceptable to the experimenters to obtain exposures using both types of focussing systems. Then a comparison could be made of the advantages of each beam. 
PROPOSAL TO STUDY DILEPTON NEUTRINO INTERACTIONS WITH THE TRIPLET QUADRUPOLE BEAM, THE PHASE I EMI, AND THE $15^{\prime}$ BUBBLE CHAMBER FILLED WITH A H-Ne MIXTURE

R. Harris, F. R. Huson, S. Kahn, T. Murphy, W. Smart (Fermilab);

R. J. Cence, F.A. Harris, S. I. Parker, M. W. Peters,

V. Z. Peterson, V. J. Stenger (Univ. of Hawaii);

A. Barbaro-Galtieri, G. Lynch, J. Marriner, M. L. Stevenson (LBL)

It is proposed to study dilepton neutrino events in the 15-foot bubble chamber using the quadrupole triplet beam. The bubble chamber would be filled with at least $80 \%$ neon by volume (a 15-ton fiducial target). The external muon identifier (EMI) would be rearranged into two planes and would have additional absorber inserted in it. With the additional absorber and the heavy neon mixture about 8-10 absorption lengths would be available for muon identification. A double plane will also enable a time coincidence to be used in identifying the muon tracks. Thus it should be possible to reduce hadron punch-through to less than $0.1 \%$. The neutrino beam would be produced with a 1 msec. spill in order to reduce backgrounds in the EMI.

The emphasis of the analysis for this experiment would be on neutrino interactions with a dimuon or a muon-electron in the final state. It is estimated that about $2 \%$ of the dilepton production will be from antineutrino interactions. The experimenters request a total of 200,000 pictures. From these there should be available 300 dimuon events and an equal number of muon-electron events.

Summary prepared by 


\section{-294- Emulsions/Protons \& $400 \quad \# 461$ \\ SEARCH FOR "NEW" PARTICLES \\ EROM $400 \mathrm{GeV}$ PROTON COLLISIONS IN EMULSIONS}

D.C. Peaslee (Australia National Univ.); P.C.M. Yock (Univ. of Auckland); V.D. Hopper (Melborne Univ.); L.S. Peak (Sydney Univ.); A.G. Fenton (Univ. of Tasmania); J.J. Lord and Richard J. Wilkes (Univ. of Washington)

It is proposed to expose stacks of nuclear track emulsions to protons of $400 \mathrm{GeV}$. The emulsions will be examined by the method of area scanning for nuclear interactions. Each interaction will be examined for evidence of unstable particles having lifetimes between $10^{-17}$ and $10^{-12}$ second. Direct evidence for particles of these lifetimes is not possible in bubble chambers but the high resolution of the nuclear emulsion makes the proposed experiment feasible.

Summary prepared by

J. J. Lord, November 1975 


\section{SEARCH FOR SHORT IIVED PARTICLES PRODUCED BY $400 \mathrm{GeV}$ PROTONS IN EMULSIONS}

W. Bozzoli, P. Capiluppi, A. Forino, R. Gessaroli, G. Giacomelli, P. Lugaresi-Serra, A. Quareni and $F$. Rimondi (Universita di Bologan); S. Di Caporiacco, A. M. Cartacci and M. G. Dagliana (Universita di Firenze)

This is a search for short-lived particles with lifetimes in the range of $10^{-12}-10^{-14} \mathrm{sec}$, performed with $400 \mathrm{GeV}$ protons in emulsion nuclei. As in experiment No. 419, the signal would be a neutral or charged decay (of possibly two decays) close to the frimary proton-nucleus interaction.

We have exposed one stack of Ilford G5 emulsions, of 80 plates of $5 \times 10 \times 0.6 \mathrm{~cm}^{3}$, to $400 \mathrm{GeV}$ protons with a density of about $3 \times 10^{4}$ protons $/ \mathrm{cm}^{2}$, with a total exposure of $1.2 \times 10^{6}$ incident protons over an area of $5 \times 8 \mathrm{~cm}^{2}$.

The experiment should be sensitive to the production of new particles if they have a mass of $1-10 \mathrm{GeV}$ and if their proeuction cross section is larger than $1 \mu \mathrm{b}$.

Summary prepared by

G. Giacomelli, January 1976 
THE INTERACTIONS OF PROTONS IN NUCLEAR EMULSION

AT $400 \mathrm{GeV} / \mathrm{C}$ (OR $500 \mathrm{GeV} / \mathrm{C}$ )

M.I. Adamovich, P.A. Cherenkov, N.A. Dobrotin, M.I. Tretjakova (Alma-Ata - Leningrad - Moscow - Tashkent Collaboration)

A request is made for an exposure of two or three emulsion stacks to a beam of $400 \mathrm{GeV} / \mathrm{C}$ (or $500 \mathrm{GeV} / \mathrm{c}$ ) protons at Fermilab. The interest in this exposure is based on the following:

1. A study of high energy proton-proton and proton-nucleus interaction and comparison with predictions from a theoretical model.

2. Measuring the energy dependence of the cross sections for production of one or more charged particles in protonnucleon and proton-nucleus interactions.

3. A study of distribution of intervals of the rapidity, containing $k-1$ particles $(k=12 \ldots n-1, n-m u l t i p l i c i t y)$ for a fixed $n$.

Similar analysis has originated from exposures by this group at Serpukhov and Fermilab and there is important to compare the results with the higher energy data.

Summary prepared by

M. I. Tretjakova, October 1975 
$30-$ Inch $\mathrm{K}^{ \pm} \mathrm{p}$ at $150 \mathrm{GeV} / \mathrm{c} \# 464$

A proposal to study diffractive processes in $\mathrm{K}^{+} \mathrm{p}$ interactions at $150 \mathrm{GeV} / \mathrm{C}$

F. Grard, V.P. Henri, P. Herquet and R. Windmolders

(Facultê des Sciences, Université de l'Etat, Mons, Belgium)

An experiment is proposed with the FNAL 30 " bubble chamber proportionnal wire hybrid system exoosed to an enriched $\mathrm{K}^{+}$beam of $150 \mathrm{GeV} / \mathrm{c}$. The number of pictures requested is $\frac{100.000}{R}$ $\left(\mathrm{R}=\mathrm{K}^{+} /\right.$contaminant ratio) to provide about $10.000 \mathrm{~K}^{+} \mathrm{p}$ interactions, corresponding to a statistics of 0.5 event/ub.

Besides other points of physics interest (topological cross-sections, elastic scattering, various inclusive analysis...) the main objective will be the study of diffractive processes in inclusive reacticns such as

$$
\mathrm{K}^{+} \mathrm{p} \rightarrow \mathrm{pX}
$$

and $k^{+} p \rightarrow k^{+} X$

as well as in 4 body and 6body exclusive reactions, of which a siqnificant number of $4 \mathrm{C}$ fits are expected. Tests of factorization will be possible by comparison with data from different interactions.

Identification of fast outgoing kaons is required for the analysis of reaction (2).

An automatic film measuring device (SWEEPNIK) will be available for this experiment.

The group is ready to collaborate with groups interested in the same experiment. (see pronosal $n^{\circ} 375$ ). 


\section{PROPOSED CHARM SEARCH WITH MUON TRIGGER}

U. Becker, J.D. Burger, M. Chen, R. Forber, F. Heimlich, D. Luckey, D. Novikoff, W. Toki, M. Weimer (MIT); D. Lowenstein (Brookhaven)

It is proposed to add a muon detector system to a double arm spectrometer (E-372) to search for (charm) pair production with high sensitivity. Should the newly discovered particles $J(3.1)$ and $\psi(3.7)$, etc. be explained as particles of hidden charm content, mesons and baryons with quantum number charm $= \pm 1$ must exist. They may be produced in pairs:

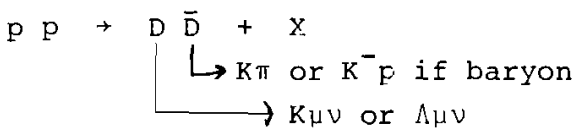

An event selection for $K \pi$ with a $\mu$ as fragment from the other charm partner will be a selective trigger for this reaction. The rate is strongly energy dependent rising a factor of $\sim 100$ from BNL to FNAL energies.

The muon detector is based on one used at BNL. It would use 20 tons of uranium absorber. It could operate under intense hadron fluxes, detect muons with $\mathrm{CM}$ momentum $\geq 700 \mathrm{MeV} / \mathrm{c}$, cover a large solid angle and absorb kaons and pions near the target to reduce background muons. A proton beam of $4 \times 10^{10}$ per pulse on a 28 collision length target is assumed.

A test set up is proposed for the spring of 1976 in the proton center beam. (No specific request for hours is made.)

Summary prepared by

w. Baker, May 1976 
A PROPOSAL FOR THE STUDY OF HIGH-ENERGY REACTION MECHANISMS BY THE MEASUREMENT OF THE ANGULAR AND ENERGY DISTRIBUTIONS OF NUCLEI RECOILING FROM TARGETS BOMBARDED WITH 200-500 GeV PROTONS

S. B. Kaufman, E. P. Steinberg, B. D. Wilkins, D. Henderson Chemistry Division, Argonne National Laboratory, Argonne, Illinois and

N. T. Porile, D. Fortney

Chemfstry Department, Purdue University, Lafayette, Indiana

and

N. Sugarman, S. K. Chang, A. Turkevich, R. A. Johns, J. LaRosa

Chemistry Department, University of Chicago, Chicago, Illinois and

L. Winsberg

Phystcs Department, University of Illinois, Chicago Circle, Chicago, Illinois

The angular distribution and the range distributions at specific angles will be measured for radionuclides which recoil out of a thin uranium target under $400-\mathrm{GeV}$ proton bombardment. These data will be analyzed in terms of the momentum transferred to the target nucleus by the incident proton and compared with similar measurements made at lower bombarding energies. The goal is to learn if the forward momentum transfer to the nucleus actually decreases with Increasing bombarding energy, as indicated by integral-recoil measur ements.

The apparatus is an evacuated scatter chamber containing the thin (ca. $0.1 \mathrm{mg} / \mathrm{cm}^{2}$ ) target and aluminum foils to catch recoiling nuclei. The beam requirements are $2 \times 10^{12}$ protons/pulse in a spot size of $4 \mathrm{~mm}$. A total of 500 hours, divided into individual runs lasting $24-48$ hours, and separated by 1-2 month intervals to allow for following the sample decay curves are requested. 
Cross section for ${ }^{\mathrm{Z}} \mathrm{A}(\mu, \mathrm{p}, \mathrm{n})^{\mathrm{Z}+1} \mathrm{~A}$ on ${ }^{37} \mathrm{C} 1$ and ${ }^{205} \mathrm{~T} 1$

Melvin S. Freedman, Charles M. Stevens, Argonne National Laboratory, and Raymond Davis, Jr., John Evans, Brookhaven National Laboratory

In the solar neutrino flux measurements via neutrino capture on ${ }^{37} \mathrm{Cl}$ of R. Davis, Jr. and that (proposed) on ${ }^{205} \mathrm{~T} 1$ by ANL, the reactions of the title generated by energetic cosmic ray muons are the principal competitive reactions producing a background of the same isotope $\left({ }^{37} \mathrm{Ar}\right.$ or $\left.{ }^{205} \mathrm{~Pb}\right)$ as does the neutrino capture. The goal of experiment $\mathrm{P}-467$ is a refined evaluation of the background in both neutino capture experiments.

By a simultaneous irradiation of 1 kilogram targets of $\mathrm{C} 1$ and $\mathrm{T} 1$ in the muon spill beam behind experiment 319, the muons first passing through a concrete proton generator, followed by off-1ine analysis at ANL and BNL for the product isotopes, the relative effective cross sections for the $(p, n)$ reactions will be measured. Calibration of the muon beam by short initial photographic plate exposure, followed by muon track counting, and on-line beam monitoring by a pair of scintillator paddles in coincidence, will yield an absolute cross section for each reaction. The muon energy range of interest is $100-300 \mathrm{GeV}$, whatever is chosen for experiment 319.

We require of the order of $10^{11}$ muons, corresponding to ca. 100 hours of bear time, as planned for experiment 319. The target mounts, in sealed containers $\left(\mathrm{C}_{2} \mathrm{Cl}_{4}\right.$, liquid, and $\mathrm{Tl}$ metal) will be housed, along with the electronics and detectors (provided), in a weatherproof wooden cubicle (provided) innediately behind two stacked $3^{\prime}$ cubical concrete blocks (request of FNAL) behind the muon building. The concrete blocks serve as muon $\rightarrow$ proton converters, simulating rock in the underground environment of the neutrino-capture experiments.

We request the assistance of the FNAL muon beam monitoring personnel in initially locating the muon beam at target setup time. We also request a 110 V.A.C., $1 \mathrm{KW}$ extension power line to our irradiation cubicle, about $30^{\circ} \mathrm{N}$. of the muon buildings. 
SEARCH FOR PENETRATING MASSIVE NEUTRAL PARTICLES PRODUCED IN HIGH ENERGY PROTON COLLISIONS

C. Y. Chang, R. W. Ellsworth, E. Sager, P. H. Steinberg University of Maryland

We propose to search for long lived neutral heavy leptons produced in high energy proton interactions in a beam dump. We request the use of the $\mathrm{E}-8$ hyperon beam magnet as the beam dump. The strong magnetic field is essential for sweeping out most of the penetrating muons produced in the hadronic showers. The heavy leptons would be detected through their decays in a 50 foot downstream decay region.

We are requesting 1000 hours for data taking and 200 hours for equipment testing. The M- 2 beam would be tuned to $400 \mathrm{GeV}$ protons (i.e. $\mathrm{x}=2 \mathrm{P}_{1} / \sqrt{\mathrm{s}^{2}} \mathrm{l}$ ) and with an intensity of $10^{9}$ protons/pulse we expect I'o events/nb/hr.

Summary prepared by

P. H. Steinberg, January 1976 


\section{SEARCI FOR HEAVY LONC-T,IVED PARTICLFS}

C. Dellarzo, L. Guerriero, P. La Vona, C. Maggi, F. Posa, C. Selvagg1, P. Sninelli.

F. haldner (Bari); D. Cutts, R. Dulude, R. Himmer, R. Janou, J. Massimo (Brown):

R. Heunier (CTRN); $\Lambda$. Brenner, n. Carev, I. Ellas. C. Mikenberg (FNAJ): W. Altkenhead, D. Barton, G. Brandenburg, Busza, J. Butler, J. Fines, J. Friedman, P. Carbincius, II. Kenda11, T. Lyons, B. Nelson, L. Rosenson, W. Toy, R. Verdier, C. Young (M.I.T.)

Utillzing the existing apparatus and unlque properties of the MGF beam Single $\Lambda \mathrm{rm}$ Spectrometer we nropose a relatively short experiment which will search for stable and long-1fved narticles which: a) Improves sensitivity over previous experiments by two orders
of magnitude;
b) extends the energy range of such searches at FNAL to $400 \mathrm{CeV}$ :
c) is sensitive to masses over a range from 1 fev to nearly the kinematic limit without exclusion of fractional or multinlv- charged narticles;
d) studies the particles (especially in the $2 \mathrm{reV}$ "antideuteron" peak) as to their hadronic interaction.

To determine mass we use both Cerenkov counter and standard time-of-flight teclininues. Five threshold and three differential Cerenkov counters are presently available and in place; with this number we can have a 1 lght particle veto with inefficiencies $210^{-10}$ and keep 5 counters free for mass measurements of heavy particles. $\Lambda$ muon detector has already been added to the Single Arm Snectrometer, so the only equinment required for this experiment not already in place (and tested) is a small number of time-of-flight counters.

We request 50 hours of setup time and 100 hours of running time with standard targeting. Under these conditions we will inspect 1010 light narticles of both signs. With the usual parametrization of the light nartfcle cross section ${ }^{\dagger}$, this total flux corresponds to an upper $11 \mathrm{~m} 1 \mathrm{t}$ for heave particle production of $4 \times 10^{-5}$ $u b / \mathrm{CeV} / \mathrm{c}-\mathrm{ster}$, per nucleon.

Summary nrepared by D. Cutts, February 1976

\footnotetext{
tWang, Phys. Rev. 17 (1973) 2609.
} 
A Measurement of the $A$-Dependence of Hadron Production

J. Hartmann, J. Orear, J. Vrieslander - Cornell University

S. Conetti, C. Hojvat, D.G. Ryan, U.G. Stairs, J. Trischuk - MCGill University

M. Gettner, W. Faissier, J. Johnson, D. Potter, E. Pothier - Northeastern University

Uivectives:

The oujective of this experiment is to measure the yield of positive inadrons produced by collisions of high energy protons with several nuclear targets distributed tinroughout tire periodic table. The measurements will be made at large Pt (from $2 \mathrm{GeV} / \mathrm{c}$ to $6 \mathrm{GeV} / \mathrm{c}$ ) and extend to the kinematic boundary. In addition to the dependence of the production cross section on atomic number, tlie yield from hydrogen will be determined as a function of $x_{1}$ and $x_{11}$.

Present data is 1 imited to regions near small $x_{\perp}$ or small $x_{i 1}$, with very limited overlap. Hadron production at large angles near the quasi-elastic region will be important in distinguishing between hadronic excitation processes and hard (constituent) scattering processes.

Technique:

Foils of $1 \%$ interaction length thickness will be exposed to the proton beam in Proton West with intensities up to $4 \times 10^{11} \mathrm{ppp} .200$ nours of beam time is required for testing and data taking. The yield of positive particles will be measured with the magnetic spectrometer constructed for E177. The spectrometer consists of a 4 meter septum magnet and two 3 meter EPB magnets. Particle trajectories are measured using multiwire proportional chambers. 
J. Hartmann, J. Orear - Cornell University

S. Conetti, C. Hojvat, D. Ryan, D. Stairs, J. Trischuk - McGill University

W. Faissler, M. Gettner, B. Gottschalk, J. Johnson, D. Potter(Spokesman) Northeastern University

\section{SUMMARY}

We propose an experiment that investigates the properties of particles produced in association with the $\psi(3.1)$ and $\psi^{\prime}(3.7)$. The physics objectives include a search for heavy parent particles which decay into the $\psi$ and multiparticle resonances produced along with the $\psi$. The latter possibility includes both a search for particles which may be "charmed" (in which case two should be produced along with the $ل$ ) as well as a search for true associated production, which may occur if the $\dot{i}$ itself has a new quantum number.

The expertment investigates both pion and proton initiated \& production processes. The differences observed in inclusive $\downarrow$ production by pions and by protons are an indication that the production mechanisms may depend on the nature of the incident: particle. For example, processes requiring anti-quarks would be more likely to be initiated by pions than by protons. Therefore, it is necessary that beams of both particles be used to search for new particles associated with the $\psi$.

The apparatus is a good resolution, large acceptance spectrometer followed by an fron muon filter. The spectrometer will be triggered by muon pairs ortginating from $\forall$ and $\psi$ ' decay and other processes, and will be used to obtain the effective mass distributions of the charged particles produced in coincidence with the $\forall$ and $\psi^{\prime}$. A large acceptance spectrometer is crucial since both the $\$$ and decay products of resonances produced along with it must be detected simultaneously. To achieve a large multiparticle acceptance with a magnet of conventional size, the thin target (1/2" beryllium) is placed inside a BMLO magnet and the particle trajectories are measured only after magnetic deflection. The resulting mass resolution for a centra11y produced $\psi$ is $140 \mathrm{MeV}$ (FWHM), which is more than sufficient to separate the $\Downarrow$ from the muon pairs due to pion decay. At $400 \mathrm{GeV}$, the acceptance for a two body decay of a particle produced at rest in the C.M.S. is about $35 \%$ 。

We propose to make the measurement in the M2 line using pion and proton beams at an intensity of 107 pulse. We will require about two hundred hours of set up time and 1200 hours of data collection time, split evenly between pion and proton beams. The expected yield of at least 5,000 proton induced $\psi$ events and at least 10,000 pion induced $\dot{t}$ events is sufficient to investigate phenomena which contribute as little as a few tenths of a percent to inclusive $\psi$ production.

This experiment is similar in purpose to E-400 but differs in two crucial respects. First, although both experiments search for new particles produced along with the $\psi$, this experiment has a much larger acceptance because the apparatus subtends about five times more solid angle in the lab. Thus, not only does it have a greater sensitivity to rare processes but it can also detect particles in kinematic regions inaccessible to E-400. Second, the pion initiated events collected in this experiment cannot be obtained by $E-400$. Consequently, the two experinents are in fact conplementary. 


\section{SEARCH FOR HEAVY PARTICLES PRODUCED IN ASSOCIATION WTTH PROMPT MUONS}

D. Bintinger, D. Jovanovic (Fermilab)

C. Akerlof, P. Alley, D. Koltick, D. Meyer, R. Thun

(Univ. of Michigan); R. Ditzler, D. Finley, O. Johnson,

F. Loeffler, E. Shibata, K. Stanfield (Purdue)

A little over one year ago FNAL approved p-357, the original proposal to search for the hadronic decays of charmed particles. During this year our group has built a powerful double-arm spectrometer which includes 32 drift chambers, 6 cherenkov counters, and 54 scintillation counters. We had our first and to date only data run in November and December of 1975. We have seen a very clean $\mathrm{J} / \psi$ signal in the $\mu^{+} \mu^{-}$decay mode and the run yielded two-body hadron data whose general experimental and statistical quality compares very favorably with the hadron data of the MIT/BNI effort.

Since this investigation concerns processes with extremely small cross sections and large physical backgrounds, we believe it to be imperative to improve the sensitivity of this particle search. Data from hadron interactions, neutrino interactions, and $e^{+} e^{-}$annihilations with prompt leptons in the final state give rather compelling evidence for the production of new particles. Therefore, we propose to search for narrow resonances in two-body hadron mass spectra for events containing a prompt muon.

Muons will be identified with a $15 \frac{1}{2}$ foot long hadron absorber built in two parts and covering more than 50 of the c.m. solid angle. An 8 foot long steel absorber with a hole for passage of the beam and channels leading to each spectrometer arm will replace the first two Cherenkov counters in our present apparatus. The second part of the absorber will utilize the iron in the BM-109 magnet yokes. The function of the two displaced Cherenkov counters will be taken over by two similar counters to be positioned behind the BM-109 magnets. 
SEARCH FOR MONOPOLES ABOVE THE 15-FOOT BUBBLE CHAMBER

D. F. Bartlett and Daniel Soo (University of Colorado), Robert L. Fleischer, Howard R. Hart, Jr, and Antonio Mogro-Campero, (Genera1 Electric Research and Development Center), M. G. White (Department of Physics, Princeton University)

Magnetic monopoles having energies less than about $40 \mathrm{TeV}$ will be slowed to their terminal velocity by the earth's atmosphere. They may then be gathered by the fringing magnetic field of the 15-foot bubble chamber. We propose placing detectors in Lexan and nuclear emulsion at colvenient locations above and in the bubble chamber. Such a system would be sensitive to monopole masses between $10 \mathrm{GeV}$ and $100 \mathrm{TeV}$ and to monopole charges between 0.7 and $10 \mathrm{hc} / 2 \mathrm{e}$.

The experiment would require the construction of a special light roof and would run for 5 weeks during a time when the bubble chamber is filled with air and not in use for other experiments. This study would lower the existing limit on inflight detection of monopoles (at the earth's surface) by a factor of twenty.

The simplicity of the detectors, the immediate availability of the experimenters, and the inability of the proposed light roof to shed snow all suggest that the experiment be run during the coming summer. The experimenters propose that such a run be scheduled during one of the long scheduled shutdowns of the 15 -foot bubble chamber. 
A DETAILED STUDY OF "EXTRA MUON" PRODUCTION IN DEEP INELASTIC MUON INTERACTIONS

B. Ball, D. Bauer, C. Chang, K. W. Chen, S. Hansen, I. Litt, J. Kiley, A. Kotlewski and P. F. Schewe (Michigan State)

We propose here a specific missing energy measurement in direct muon production by high energy muons. First we briefly summarize heretofore unpublished dimuon data collected in a previous muon scattering experiment (E-26). These data support a possible new particle production mechanism. Forty events were found to show extra muons accompanying the scattered muon. The se extra muon(s) could conceivably be interpreted as the leptonic or semileptonic decays of some new hadron(s) produced in high momentum-transfer muon interactions. The rate for the extra muon production is greater than $2 \times 10^{-3}$ of single $\mu$ events.

The proposed experiment will be performed using the E-319 apparatus, consisting of a newly constructed fine-grained hadron calorimeter (240" in length and 1-7/8" Fe sandwiched by 1/4" plastic scintillators), an improved muon spectrometer and new beam proportional chambers to be shared with the $E-398$ group. This new apparatus is particularly well suited to observe the production and weak decays of new hadrons. Among the unique advantages in the detail studies of mo-production of new particles include 1) a precise knowledge of kinematics: incoming muon energy, $E_{O}$, scattered muon energy $E^{1}, Q^{2}, v, x$, and $W$, etc., 2) a good measurement of missing energy carried by neutrinos, 3) a large solid angle acceptance for final state muons, 4) an experimental verification of "promptness" of extra muon yields using varying target densities and 5) flexibility in trigger with three sets of new trigger counter hodoscopes.

A run of $10^{11}$ muons is requested at $E_{\mu}=300 \mathrm{GeV}$ with an intensity of $10^{6}$ muons/pulse. This run can be carried out in the same quadrupole-triplet running period as $E-319$ following data taking by E-398. We anticipate collecting 2300 dimuon events.

We emphasize here that our improved apparatus resembles that proposed in proposal E-225 (1973), in which the original idea of using a target-calorimeter to stuay high momentumtransfer interactions was advanced for the first time. The fact that we were able to observe dimuon events with high efficiency using E-2.6 apparatus demonstrates that our apparatus will be more than adequate to search for the existence and to study the reak decay of new particles. 
SEARCH FOR MULTIPHOTON EVENTS PRODUCED IN PHOTON-BERYLLIUM INTERACTIONS

G. B. Collins (VPI \& SU), J. R. Ficenec (VPI \& SU), J. Fischer (BNL), W. P. Trower (VPI \& SU)

The purpose of this experiment is to continue our search for multiphoton events at FNAL. Multiphoton events have been observed by several authors in cosnic ray exposures in the 1950's and have been associated by Ruderman and Zwanziger with bound magnetic monopole pairs. In our initial search, Multigamma \$22, we sought to produce anomalous multiphoton events using $300 \mathrm{GeV} / \mathrm{c}$ protons on beryllium. In Multigamna $\$ 22$, after subtracting tbe neutron background and accounting for the known sources of photons, there remains a residue of 11 multiphoton events whose cross section is most consistent with production by photons and whose characteristics are similar to the reported cosmic ray events. We find that each event has 5-7 converted photons iminediately behind a $0.57 \mathrm{rl}$ $\mathrm{Pb}$ converter, a projected production angle whose centroid is $0.1-2.4 \mathrm{mr}$, a production cone angle of $0.5-5.8 \mathrm{mr}$, and a total electromagnetic energy $>100 \mathrm{GeV}$.

We propose to continue our search for anomalous multiphoton events in the tagged photon beam at FNAL because these events are most probably produced in photon interactions, and the tagged beam has the advantage of known photon intensity, angle and energy free of the troublesome neutron background. Thus the interpretation of the experimental results is less speculative. The experimental arrangement would optimize our search for these narrow, multiphoton showers and would benefit in its design from the experience gained in our previous search. The multiphotons would be produced in a Be target, travel $\sim 40 \mathrm{~m}$. in vacuum, and form showers in a thick $\mathrm{Pb}$ converter. The shower cores would be recorded in desensitized multiwire proportional chambers (MIPC's) and their direction would be determined by conventional MWPC's. $\Lambda$ lead glass counter array would be used to determine the total energy of the multiphotons. A candidate event would be required to have several shower cores and a total multiphoton energy nearly equal to the incident photon energy.

If we are scheduled to immediately follow E25A, and are able to use some of their existing apparacus (24" vacuum pipe, lead glass array, and information collection system), we would require only two weeks of cesting and 100 hours of data-taking to complete the experiment. In order to be effective, each week of testing or data-taking should be preceded by a week with no beam usage. These estimates assume that $3 \times 10^{4}$ electrons/burst at $300 \mathrm{GeV} / \mathrm{c}$ are available in the tagged photon laboratory. With a two percent radiator in the tagging system, this intensity gives 400 photons/pulse with energies > $133 \mathrm{GeV}$. Using our estimated cross section for multiphoton production in $\gamma$-Be interactions [(0.2-10.8) mb], > 400 multiphoton events should be recorded, allowing cross sections as a function of energy, multiplicity, and production angle to be measured. 


\section{PROPOSAL FOR STUDYING ANTI-NEUTRINO INTERACTIONS \\ IN NEON WITH A NARROW BAND BEAM IN THE 15-FOOT BUBBLE CHAMBER}

S. Y. Fung, A. Kernan, R. T. Poe and B. C. Shen (UC Riverside);

W. M. Smart (Fermilab); J. R. Albright, S. Hagopian, V. Hagopian, J. Lannutti (Florida State)

This proposal is for the study of anti-neutrino interactions with a dichromatic anti-neutrino beam in the 15-ft bubble chamber filled with neon or a heavy neon-hydrogen mixture. A total of 200,000 pictures is requested. For $400 \mathrm{GeV}$ protons at intensity 1013 protons per pulse we expect approximately 4000 anti-neutrino interactions, of which about 1000 are neutral current interactions. The primary purpose of the proposed experiment is to make a detailed study of neutral current phenomena.

Summary prepared by

A. Kernan, January 1976 


\section{STUDY OF $\pi, K$, AND $\bar{p}$ PRODUCTION OF HIGH MASS MULTI MUON STATES}

W. Faissler, P. Gauthier, J. Johnson, M. Mallary, E. Pothier, E. von Goeler, R. Weinstein (Northeastern University)

The reactions $(\pi, K, \bar{p})+F e+$ muons + anything will be observed in order to measure $\sigma$ and its dependence on $x$ and $p_{1}$, and to compare to equivalent proton production cross sections. For example, a dimuon resonance at $7 \mathrm{GeV}$, with a production cross section 1000 times smaller than the $\psi$ would result in a yield of 200 events. A beam dump method with improved resolution is employed. 400 hours of data taking and 100 hours of testing with 200 to $250 \mathrm{GeV}$ pions at an intensity of $10^{7} \pi$ /pulse is requested.

Summary prepared by

R. Weinstein, January 1976 
PROPOSAL TO SEARCH FOR INTERMEDIATE BOSON PRODUCTION IN PROTON PROTON COLLISIONS AT $200 \mathrm{GeV}$ IN THE CENTER OF MASS

R. Huson, P. Livdahl, R. Steining, L. Teng, E. Turkot, J.K. Walker (Fermilab)

It is proposed to construct a $25 \mathrm{GeV}$ proton storage device at straight section $E$ of the main ring. This will permit collisions between the stored protons and the normal accelerated beam in the main ring during each acceleration cycle. In Phase I of the experinient CMS energies up to $200 \mathrm{GeV}$ and luminosities up to $10^{31} \mathrm{~cm}^{-2} \mathrm{sec}^{-1}$ will be achieved. A second phase will raise the energy to $400 \mathrm{GeV}$ in the CMS and increase the luminosity to $10^{32} \mathrm{~cm}^{-2} \mathrm{sec}^{-1}$.

Toroidally magnetized iron slabs interspersed with drift chambers and scintillation counters will surround the $18 \mathrm{~m}$ long intersection straight. Large transverse momentum muons will be used in the trigger. A study of the single and multiple production of muons will be made. Imbalance in transverse momentum of the hadronic cascades will signify neutrinos in the final state.

\pm A search will be made for a new energy scale signified by $\mathrm{w}^{ \pm}, \mathrm{Z}^{\circ}$, Higgs bosons, quarks and heavy lepton production. Typically, 100 detected events per week are expected for $60 \mathrm{GeV}$ mass charged intermediate boson (W) production.

The operation of this experiment would be completely parasitic to the normal operation of the research program in the same way as $\mathrm{Co}$. 


\section{NEUTRON-DEUTERON ELASTIC SCATTERING}

T. Roberts, Cyril A. Ayre, H. Richard Gustafson, Lawrence W. Jones, and Michael J. Longo (University of Michigan)

We propose to measure the neutron-deuteron differential elastic cross-section in the region $0.25 \leq t \leq 1.5(\mathrm{GeV} / \mathrm{c})^{2}$ for $100 \leq \mathrm{P} 1 \mathrm{ab} \leq 400 \mathrm{GeV} / \mathrm{c}$. The equipment requires is identical to that currently in use by $E-248$, with deuterium replacing hydrogen in the target. Three hundred hours of beam (in the $M-3$ beam line) are requested. This experiment would not be undertaken until E-248 is completed. 
PROPOSAL TO SEARCH FOR HEAVY BOSONS, HEAVY LEPTONS, AND CHARMED PARTICLES AT SSR

Luke W. Mo (University of Chicago); Peter E. Schlein (UCLA); Andris Skuja (University of Oxford)

This is a proposal to look for heavy bosons, heavy leptons, and charmed particles using a small storage ring (SSR; see Fermilab proposal \#478). The three processes to be investigated are:

(1)

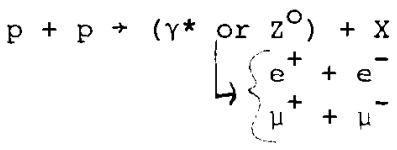

(heavy bosons)

(2)

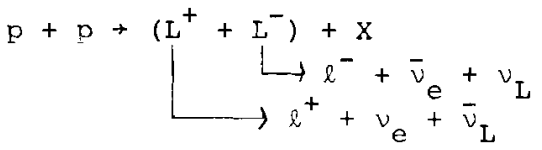

(heavy leptons)

(3)

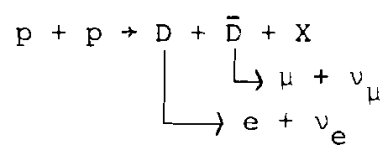

(charmed particles)

The signature for the processes are characterized by charged lepton pairs in the final state. Hadrons associated with the heavy particle production will be measured. It should help to determine the properties of these new particles.

The spectrometer proposed here consists of a wheel-shaped superconducting magnet, sets of drift chambers, scintillation counter hodoscopes and a lead glass hodoscope.

Under certain assumptions (parton model, etc.) they expect the integrated counting rate of $\sim^{-2}$ events $/ \mathrm{GeV} / \mathrm{hour}$ for $10 \mathrm{GeV} / \mathrm{c}^{2}$ and $\sim 10^{-3}$ for $20 \mathrm{GeV} / \mathrm{c}^{2}$.

One year of running time on the $S S R$ is requested. 
$$
-314-
$$$$
\text { Emulsions } / \pi^{-} \text {a } 200 \text { \#481 }
$$ \\ INVESTIGATION OF MULTIPLE PRODUCTION BY $\pi^{-}$MESONS WITH EMULSION CHAMBER}

Y. Takahashi, M. Biyajima, T. Matsuda and O. Miyamura (Osaka University)

This is a study of multiparticle production using $\pi^{-}$of $200 \mathrm{GeV}$ or higher and nuclear emulsions. The emulsion chamber consists of two parts. The top half consists of a stack of emulsion plates and thebottom half contains emulsion, x-ray film and leads plates for gamma conversion. Each emulsion chamber measures $10 \times 10 \times 15 \mathrm{~cm}^{3}$ and a total of ten are to be exposed. The desired exposure is about $10^{4} \pi^{-} / \mathrm{cm}^{2}$.

Summary prepared by

J. Lach, May 1976 
STUDY OF DIMUON EVENTS PRODUCED IN NEUTPINO INTERACTIONS

B.C. Barish, J.F. Bartlett, A. Bodek, K.W. Brown, D. Buchholz, F.S. Merritt, F.J. Sciulli, L. Strutte, H. Suter (Caltech); E. Fisk and G. Krafczyk (F'ermilab)

This proposal is an outgrowth of E356 and represents that Fart of the experiment directed to the detection of dimuon events. The experiment will be carried out in Lab $E$ and will be done with a subset of the apparatus being constructed for E356.

With the quad beam focus set to $200 \mathrm{GeV}$, in a 75 ton fiducial volume (of the full volume of 170 tons), the charged current rate will be about 1 event/minute for $E_{>}>100 \mathrm{GeV}$ at $10^{13}$ ppp and 10 second repetition rate. For a $1 \%$ dimuon rate, they feel a realistic goal might be 250 dimuon events/ month. 
PROTON-PROTON ELASTIC SCATTERING AT $|t|=374(\mathrm{GeV} / \mathrm{C})^{2}$

Cyril A. Ayre, H. Richard Gustafson, Lawrence W. Jones, Michael J. Longo, Thomas J. Roberts (University of Michigan)

We propose to measure (or put a stringent upper limit on) the differential cross section for $\mathrm{p}$-p elastic scattering at $90^{\circ}$ in th CMS with incident protons of $400 \mathrm{GeV}$. This would push the frontier of high-energy physics out to $t=-374$ $(\mathrm{GeV} / \mathrm{C})^{2}$, more than an order-of-magnitude increase over the range of previous experiments. This corresponds to a distance scale $\ell \equiv \hbar / \mathrm{p}_{\mathrm{T}}$ which is $0.015 \mathrm{fm}$.

The experiment is straightforward and modest in scale. The apparatus consists of alliquid hydrogen target and two well-shielded spectrometers placed symmetrically about the incident proton beam. Each spectrometer contains two $20 \mathrm{ft}$. main ring magnets, proportional chambers (or counter hodoscopes), and a total absorption calorimeter.

We anticipate a sensitivity $(\mathrm{d} \sigma / \mathrm{d} t) \min \sim 10^{-41} \mathrm{~cm}^{2} /(\mathrm{GeV} / \mathrm{c})^{2}$. This estimate is based on a running timemin of $600 \mathrm{hrs}$ at 608 overall efficiency with $3 \times 10^{12}$ protons/pulse.

Summary prepared by M. Longo/J. Lach, May 1976 
PROPOSAL TO STUDY $\psi$ PRODUCTION USING THE E-416 APPARATUS

T. Burnett, V. Cook, S. Csorna, D. Holmgren, A. Jonckheere, H.J. Lubatti, K. Moriyasu (University of Washington); D. Fournier, P. Heusse, P. Rancon, J.J. Veillet (Orsay)

A number of experiments have been performed at FNAL to study hadronic production of $\psi$ mesons by observing the $\mu^{+} \mu^{-}$decay mode. These experiments have mainly concentrated on the production of the $\psi$. The momenta of the muons were determined, from which the Feynman $x$ and $P_{T}$ distributions of the $\psi$ production are obtained. The hadrons which are produced in association with the $\psi$ mesons have been either ignored or not very well studied.

We are proposing to study the reaction $\pi^{-}+$Lucite $\left(\mathrm{C}_{5} \mathrm{H}_{8} \mathrm{O}_{2}\right) \rightarrow$ $\psi+$ anything using the apparatus of E-416. The target is placed at the upstream end of a streamer chamber which is in an approximately $8 \mathrm{~kg}$ magnetic field. Downstream of the streamer chamber magnet there is a muon spectrometer containing magnetized iron and eight muon telescopes which allows us to trigger on 1, 2 or more muons in the final state. The $\psi$ mesons can be selected by reconstructing the mass of the $\mu^{+} \mu^{-}$pairs from the bend information obtained in the SOD magnet. The apparatus is icentical to that used in E-416. The only changes we propose to make to the apparatus of $\mathrm{E}-416$ in this experiment is to: (i) improve the multitrack efficiency of the spark chambers at the downstream enc. of the muon telescope; (ii) include a small multiwire proportional chamber between the downstream end of the streamer chamber magnet and the upstream end of the muon filter; (iii) close the $\pm 9 \mathrm{mr}$ gap in the muon telescopes.

We estimate that there should be a factor of 30 increase in the number of $\psi^{\prime} s$ we can obtain in this run. From the seven events in the $\psi$ region obtained in $\mathrm{E}-416$ we then estimate approximately $200 \psi^{\prime} s$ in this experiment.

From the streamer chamber we will obtain the momentum spectrum of the $V^{\circ}$ 's observed in the chamber, the rapidity of all of the charged particles, and themultiplicity distributions of charged particles. We would expect to have on the order of 100-200 events from interactions in the target and an equal number in the $\mathrm{Fe}$ shield in this experiment. We stress the fact that it requires essentially no new equipment; it simply requires reinstalling equipment with which we have had considerable experience, namely the streamer chamber and the muon telescope.

The low mass (below the $\psi$ ) muons are also of considerable interest to resolve the question of Drell-Yan verses other production mechanisms (e.g. Farrar-Frautschi). Having information about the hadrons which are produced in association with these low mass pairs may help in understanding the production mechanism.

Summary prepared by

H.J. Lubatti/W.F. Baker, May 1976 


\section{$-318-$ \\ Charged Hyperon \#485}

A PROPOSAL TO INVESTIGATE EXOTIC REGGEON EXCHANGE IN THE INCLUSIVE PRODUCTION OF CHARGED IIYPERONS

W.E. Cleland, W.E. Cooper, E. Engels, P.F. Shepard, J. Thompson (University of Pittsburgh)

We propose to measure the inclusive production of the charged hyperons $\Sigma^{-}$and $\Xi^{-}$in the reaction $\mathrm{p}+$ nucleon $\left(\Sigma^{-}\right.$, or $\left.\equiv^{-}\right)+M_{X}$ as a function of the variables $s, t$, and $M_{x}^{2}$. An analysis of the data using triple Regge theory will measure the trajectory, $\alpha(t)$, of the exchanged Reggeon for $t$ values $-5 \mathrm{GeV}^{2} \leq t \leq 0 \mathrm{GeV}^{2}$. The exchange is exotic, characterized as a doubly-charged meson with $S=+1$ and +2 for $\Sigma$ and $\equiv$ - production, respectively. A measurement of $\alpha(0)$ and $\alpha^{\prime}(0)$ consistent with triple Regge results for non-exotic exchanges lends support for the existence of exotics, and will yield definite experimental predictions of the possible masses and spins of exotic mesons to bc searched for as s-channel resonances. The polarization of the charged hyperons will also be measured.

We have designed the experiment around existing laboratory magnets. We request that the laboratory supply two BMl09's, five EPB magnets, standard fast electronics, and the necessary shielding for the channel.

We would require approximately 500 hours of beam time to do the experiment. It would be our intention to take the bulk of the data at an incident beam energy of $400 \mathrm{GeV}$ using an intensity of between 1010 and $10^{11}$ protons/pulse. Because of the triple Regge prediction that the cross sections scale as $s$, we would like to do some running at 200 and $300 \mathrm{GeV}$ using a "front porch" mode of accelerator operation.

Summary prepared by

P. F. Shepard, J. Lach, May 1976 
PROPOSAL TO STUDY THE ATOMIC NUMBER DEPENDENCE OF

THE DIFFERENCE BETWEEN PARTICLE AND ANTI-PARTICLE' TOTAL CROSS-SECTIONS

J.Hoffnagle, W. Molzon, J. Roehrig, V.L. Telegdi, E. Winstein (University of Chicago); A. Gsponer (ETH Zurich); S. Aronson, G. Bock (Univ. of Wisconsin)

We propose to perform a high statistics, virtually systematic free measurement of the $A$-dependence of the difference in particle/anti-particle total cross-sections by use of coherent regeneration in a "double beam" arrangement in the M4 neutral beam-line. One beam will be used for monitoring, the other will contain the targets. The roles of the beams will be interchanged on a pulse-by-pulse basis. By the same technique, in good geonetry, we will obtain precision neasurements of the $\mathrm{K}_{\mathrm{L}}$-Nucleus total cross-sections.

Anomalous behavior in the cross-section difference might be expected as the contributing channels are becoming rarer with energy (the cross-sections approach each other with energy): if the difference goes like $A \beta$ might move from 0.67 to 1.0 as the momentum increases. We will measure 8 to an absolute accuracy of better than \pm .01 in several momentum bins between 30 and $150 \mathrm{GeV} / \mathrm{c}$. Data taking vill require $1.4 \times 10^{17}$ protons incident on the meson target $(\cong 200$ hours $)$. 
A PROPOSAL TO S'TUDY THE ORIGINS OF "EXTRA" PROMPT MUONS PRODUCED IN DEEP INELASTIC MUON-NUCLEON SCATTERING

ฟi. A. Loomis, A.L. Sessomis, C. Tao, R. Wilson (Harvard University); H.L. Anderson, S.C. Wright (Enrico Fermi Inst.); T. Kirk, W.A. Francis (University of Illinois)

This is an experiment to study "extra" muon production in muon-nucleon interactions. Using the apparatus proposed for $E-398$ this group propose to construct an experiment highly sensitive to multimuon events. If current ideas about quark structure, and their use in explaining the reutrino dimuon results, are valid one expects the pair production of charmed particles, and their subsequent semileptonic decay, in the reaction

$$
\mu^{+}+N \rightarrow \mu^{+} c \bar{c}^{\bar{c}+\text { hadrons }} \begin{aligned}
& \longrightarrow \mu^{-}+\bar{v}_{\mu}+\ldots \\
& \rightarrow \mu^{+}+v_{\mu}+\ldots
\end{aligned}
$$

Under certain assumptions the ratio of dimuon (trinuon) to single muon events is estimated to be $210^{-3}\left(\geq 10^{-4}\right)$. Trimuon of the type $\mu^{+} \mathrm{N} \rightarrow \mu^{+} \mu^{+}{ }^{+} X$ are not adritted by any charmed mechanism and, if seen in reasonable numbers, would discredit charm as the explanation of multi-muon events.

Given that sufficient numbers of multi-muon events are recorded it will be possible to look for threshold effect as a function of $S=2 M \nu+M^{2}-Q^{2}$.

They will also study, via calorimetry, whether events with multiple muon have anomalous amount of "missing" energy. The recent results of the Yale-Brookhaven-Fermilab collaboration incicates that prompt muons produced in proton-nucleon collisions originate primarily from electromagnetic sources. If it is also true of "extra" muons produced in muon-nucleon scattering the dimuon events will be similar to the "normal" muon events, i.e., there will be no anornalous "missing energy".

A run about $3.6 \times 10^{11}$ muons on target at $225 \mathrm{GeV} / \mathrm{C}$ is requested.

Summary prepared by

T. Yamanouchi, MaY 1976 


\section{PROPOSAL FOR A STUDY OF HEAVY PARTICLE PRODUCTION AND DYNAMICS NEAR $X=0$ AND THE DEPENDENCE ON INCIDENT QUANTUM NUMBERS}

D. Brick, M. Heller, A.M. Shapiro, M. Widgoff (Brown University); R. Burnstein, C. Fu, F. Rubin (Ill. Inst. of Technology); E.D. Alyea, Jr. (Indiana University); B. Barnett, C.-Y Chien, P. Lucas, A. Pevsner, R. Zdanis (Johns Hopkins University); J. Erau, J. Grunhaus, E.S. Hafen, R.I. Hulsizer, U. Karshon, V. Kistiakowsky, P. Miller, A. Napier, I.A. Pless, J.P. Silverman, R.K. Yamanoto (Nassachusetts Inst. of Technology); F.o. Cohn (Oak Ridge National Laboratory); B. Denby, P. Jacques, T.C. Ou, R.J. Plano, T.L. Watts (Rutgers University); E.B. Brucker, E. Koller, P. Stamer, S. Taylor (Stevens Inst. of Technology); C.R. Sun (SUNY, Albany); W.M. Bugg, C. Condo, T. Handler, E. Hart (University of Tennessee); H. Kraybill, D. Ljung, T. Lualam, H.D. Taft (Yale University)

We propose a study of the production of heavy particles $\left(\Lambda^{\circ}, K^{\circ}, K^{+}, K^{-}, p, \bar{p}\right)$ with a tagged positive $300 \mathrm{ceV} / \mathrm{c}$ beam incident on the Fermilab hybrid spectrometer. We propose both a positive and negative exposure. We wish to achieve a positive beam composition $\mathrm{p} / \pi^{+} / \mathrm{K}^{+}$ratio of $6 / 3 / 1$ and a negative beam composition of $\bar{p} / \pi^{-}$of $1 / 4$. We propose $1.5 \times 10^{6}$ positive beam pictures and $1.0 \times 10^{6}$ negative beam pictures. We would use the downstream particle identifier to be chosen by the 7-8 May 1976 workshop. The major objective of the experiment is to study the role heavy particles play in multiparticle production processes at Fermilab energies. This process would be studied as a function of the projectile particle urder identical experimental conditions. This will be the first time such complete cata will be available. In addition to this new data, the experiment will yield a major increase in statistics over earlier, rore conventional studies. If it required a lower beam momenturn to achieve the desired particle ratio, we would perform the experiment at that lower beam momentur. 
PROPOSAL TO STUDY NEUTRINO INTERACTIONS IN HYDRGGEN AND NUCLEI WITH AN INTERNAL TARGET AND CONVERTER SYSTEM IN THE 15-FT. BUBBLE CHAMBER

J.P. Berge, F.A. DiBianca, P. Hanft, F.A. Nezrick, G.W. Scott, W. Smart (Fermi National Accelerator Laboratory)

We propose a study of broad-band neutrino interactions on free protons and simultaneously on nuclei with $90 \%$ gamma conversion, $95 \%$ electron and muon identification by using an Internal Target and Converter (ITC) system in the 15-ft. chamber. We request an exposure of 300,000 pictures with $1.3 \times 10^{13}$ protons per pulse of energy $400 \mathrm{GeV}$. Eased on measured yielas from E-45 we expect 100,000 neutrino events in this exposure. The physics program includes studies of $\mu^{-} e^{+}$and $\mu^{-} \mu^{+}$production; searches for charmed particles, $\psi^{\prime} s$, heavy leptons, intermediate vector bosons and other new particles; comparisons of $v p$ and vn interactions including precision measurements of $\sigma(n) / \sigma(p)$ for both charged and neutral currents; charged and neutral current deep inelastic scattering; detailed studies of exclusive channels including $\pi^{\circ} \mathrm{S}, \mathrm{V}^{\circ} \mathrm{S}$ and kinks in both charged and neutral current reactions; pure leptonic interactions; and charged and neutral current reactions; pure leptonic interactions; and charged and neutral particle multiplicity distributions in both charged and neutral current reactions.

Summary prepared by F. A. Nezrick, May 1976 


\section{SEARCH FOR SHORT LIVED FARTICLES LISING}

A HIGH RESOLUTIOIN STREAMEF. CHAMBER

1. Dine, D. Ljung, T. Ludlam, R. Majka, J. Marx, P. Nemethy, J. Sandweiss, A. Schiz, J. Slaughter, H. Taft (Yale University); M. Atac, S. Ecklund (Fermilab)

We propose to search for new short lived particles procuced in hadronic collisions in a manner independent of decay mocie, with $\sim 4 \pi$ geometry and with a sensitivity such that cross sections comparable to those for $\amalg / J$ production would be successfully detected.

Using a small triggered high resolution ( $\leqslant 10 \mu \mathrm{m})$ high pressure streamer charber, currently under construction at Yale, ve will be able to directly observe particles with lifetimes as short as $2.5 \times 10^{-14}$ sec. If such particles exist we vill measure their lifetimes, ciecay multiplicities, semi-leptonic branching ratios and production cross sections. We will observe phenomena associated with the production of these particles such as associated production or pair production, the topological and kinematical character of the events in which the new particles are produced, and any new "short time" phenomena asscciatec with the new particles (such as decays of new heavy mesons into heavy leptons). If such particles do not exist we will set a significant upper limit on $c$ times the branching ratio for semi-leptonic decays of $\sim 10$ to $50 \mathrm{nb}$ for lifetimes from $2 \times 10^{-14} \mathrm{sec}$ to $5 \times 10^{-13} \mathrm{sec}$.

We need a $200 \mathrm{GeV} / \mathrm{c}^{-}$beam at an intensity of $8 \times 10^{5} / \mathrm{spill}$. The floor space required, exclusive of accelerator and beam 1 ine, is about $2 \mathrm{~m} \times 2 \mathrm{~m}$. Wie are asking for 800 hours of beam time which will result in $7 \times 10^{5}$ pictures, civing $1.6 \times 10^{5}$ interactions in the fiducial volume of the chanber. This will give a sensitivity to particles produced with cross sections comparable to $\psi / J$. 


\section{CLASHING GIGANTIC SYNCHROTRONS}

C. M. Ankenbrandt, T. L. Collins, H. E. Fisk, R. P. Johnson, P. Limon, J. Peoples (Fermilab); A. V. Tollestrup, R. I. Walker (CalTech); L. M. Lederman (Columbia)

This experiment is a search for intermediate vector bosons, $\mathrm{w}^{ \pm}$and $\mathrm{w}^{\circ}$, and high transverse momentum phenomena produced at very large center of mass energies. These energies are made possible by colliding the beam in the Main Ring with the beam in the 1000 GeV superconducting Energy Saver/Doubler.

A collision scheme and a general purpose detector have been designed to allow the simultaneous detection of:

$$
\mathrm{p}+\mathrm{p}+\mathrm{w}^{ \pm}+\underset{\mathrm{L} \text { hadrons }}{\longrightarrow \text { Anything }}
$$

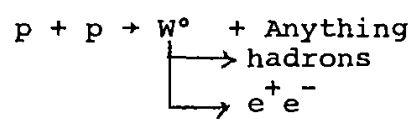

$$
p+p+\text { hadrons }\left(P_{t}>5 \mathrm{GeV} / \mathrm{c}\right)+\text { Anything. }
$$

The MR operating at $150 \mathrm{GeV}$ and the ES/D operating at $1000 \mathrm{GeV}$ with one beam traveling in the reversed direction are made to intersect at the middle of a long straight section to provide a center of mass energy $\sqrt{ }=770 \mathrm{GeV}$ or 1.2 ergs. By powering the quadrupoles at the ends of the straight section appropriately, very low $\beta$ values are obtained at the intersecting region resulting in a luminosity of $2 \times 10^{30} \mathrm{~cm}^{-2} \mathrm{sec}^{-1}$ for beams of $2 \times 10^{13}$ protons in the ES/D and in the MR. Further luminosity improvements are likely.

Because the beams are rf bunched, the interaction region is localized $\left(0.1 \times 0.1 \times 20 \mathrm{~cm}^{3}\right)$ and the detector is correspondingly compact. The detector uses dxift chambers, electromagnetic shower detectors and hadron calorimeters. It is modular and can be rearranged to be compatible with different beam energies in the MR or Doublex. With the $150 \mathrm{GeV}$ on $1000 \mathrm{GeV}$ scheme the detector has identical arms on either side of the interaction region, each centered about $90^{\circ}$ in the C.O.M. $\left(42^{\circ}\right.$ in the $\left.1 \mathrm{ab}\right)$ and each covering $\sim .6 \pi$ steradians in the C.O.M. Because of the small and uncertain $W$ branching ratio to leptons, the detector is sensitive to hadronic as well as leptonic decay modes.

Calculations based on a Drell-Yan model with colored quarks indicate that the experiment described here will be sensitive to W's weighing as much as $200 \mathrm{GeV}$, and the expected rate for detecting 100 GeV W's is of the order of ten events per day.

Surmary prepared by:

R. P. Johnson

May 1976 